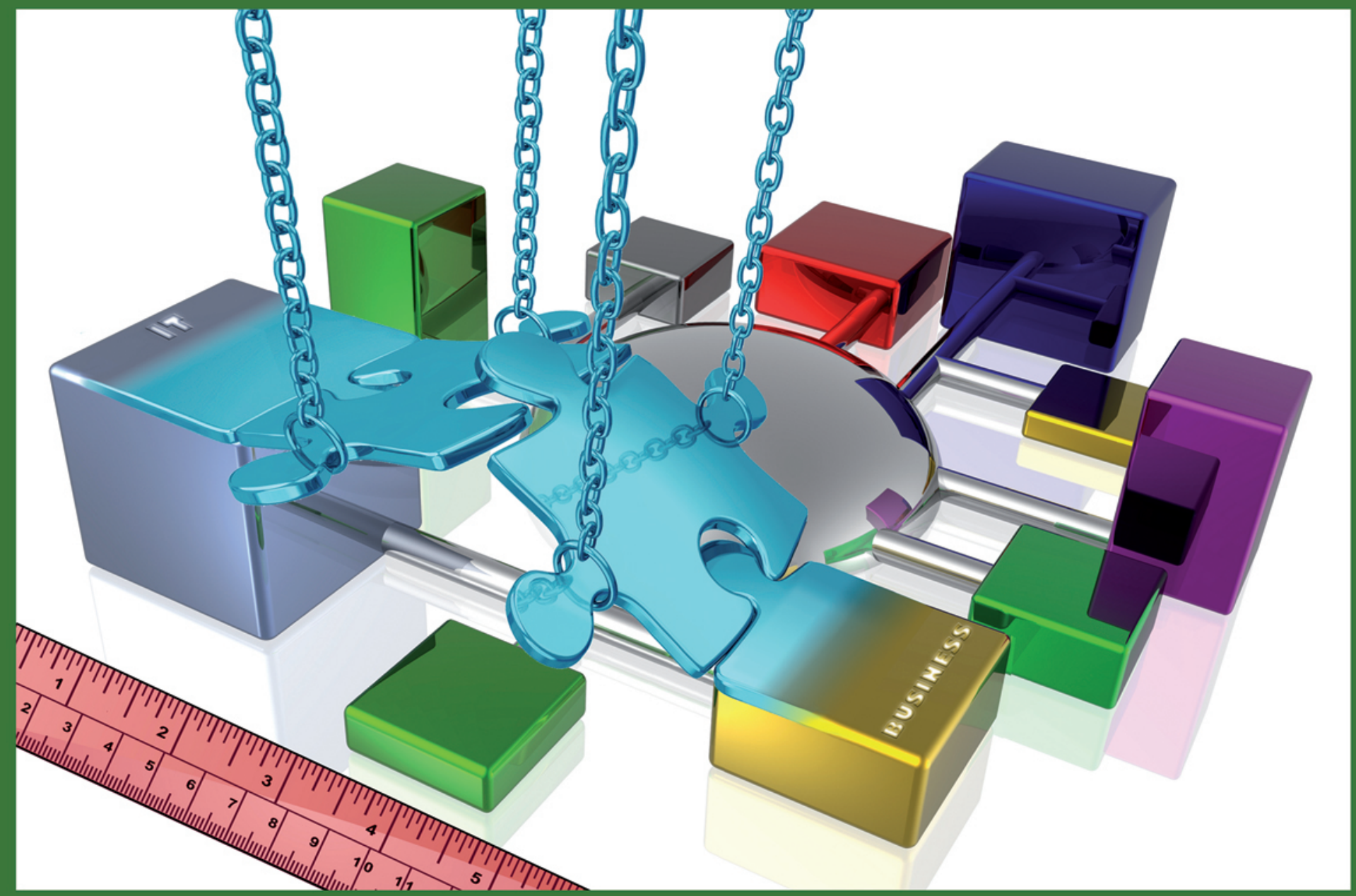

\title{
Assessing business-IT alignment in networked organizations
}

Roberto Gpe. Santana Tapia

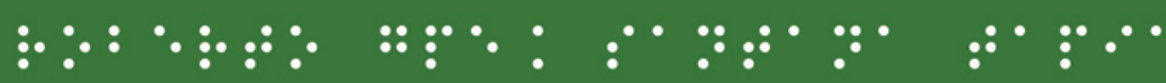




\section{ASSESSING BUSINESS-IT ALIGNMENT IN NETWORKED ORGANIZATIONS}

Roberto Guadalupe Santana Tapia 
Ph.D. dissertation committee:

Chairman and secretary

Prof. dr. ir. A. J. Mouthaan University of Twente, the Netherlands Promotor

Prof. dr. R. J. Wieringa University of Twente, the Netherlands

Assistant promotors

Dr. P. A. T. van Eck University of Twente, the Netherlands

Dr. M. Daneva University of Twente, the Netherlands

Members

Prof. dr. ir. M. Aksit

Dr. A. L. Opdahl

University of Twente, the Netherlands

Prof. dr. H. A. Proper

University of Bergen, Norway

Dr. C. Salinesi

Radboud University Nijmegen, the Netherlands

University Paris 1 Pantheon Sorbonne, France

Prof. dr. J. van Hillegersberg University of Twente, the Netherlands

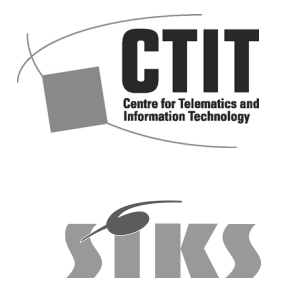

CTIT Ph.D. Thesis Series No. 09-155

Centre for Telematics and Information Technology

P.O. Box 217, 7500 AE

Enschede, the Netherlands

SIKS Dissertation Series No. 2009-44

The research reported in this thesis has been carried out under the auspices of SIKS, the Dutch Research School for Information and Knowledge Systems.

\section{$\sqrt{10}$ \\ Netherlands Organisation for Scientific Research}

This research was supported by the Netherlands Organisation for Scientific Research (NWO) under project number 638.003.407 (Value-Based Business-IT Alignment).

Printed and bound by Ipskamp Drukkers B.V.

ISBN: 978-90-365-2927-3

ISSN: 1381-3617 (CTIT Ph.D. Thesis Series No. 09-155)

http://dx.doi.org/10.3990/1.9789036529273

Copyright (C) 2009, Roberto Guadalupe Santana Tapia

All rights reserved. No part of this book may be reproduced or transmitted in any form or by any means, electronic or mechanical, including photography, recording, or any information storage and retrieval system, without prior written permission of the author.

Cover designed by Roberto Guadalupe Santana Tapia van den Berg using images downloaded from http://www.dreamstime.com (photographers Dmitry Sunagatov \& Yudesign). 


\title{
ASSESSING BUSINESS-IT ALIGNMENT IN NETWORKED ORGANIZATIONS
}

\author{
DISSERTATION
}

\author{
to obtain \\ the degree of doctor at the University of Twente, \\ on the authority of the rector magnificus, \\ prof. dr. H. Brinksma, \\ on account of the decision of the graduation committee, \\ to be publicly defended \\ on Friday, December 4, 2009 at 15.00
}

by

Roberto Guadalupe Santana Tapia

born on February 18, 1975

in Matamoros, Tamaulipas, Mexico 
This dissertation has been approved by:

Prof. dr. R. J. Wieringa (promotor)

Dr. P. A. T. van Eck (assistant promotor)

Dr. M. Daneva (assistant promotor) 


\section{Abstract}

Concerns such as identifying ways to control costs, improve quality, increase effectiveness, and manage risk have become increasingly important for organizations as they face more and more pressure to gain and maintain their competitive edge. Business-IT alignment (B-ITa) is recognized as a solution to these concerns. Aligning IT with the business remains one of the top priorities for both business practitioners and researchers. Interest in B-ITa is stimulated by cases of organizations that have successfully aligned their IT to gain competitive advantage and to improve organizational performance.

There is a considerable literature on B-ITa in single organizations. Within this broad scope of literature, a number of authors have stressed the importance of assessing B-ITa in order to plan B-ITa improvement actions. In support of this, these authors have developed maturity models (MMs). MMs describe the development of a specific domain over time. Based on maturity assessments, organizations know the extent to which processes in such domains are predictable. That is, organizations can be aware of whether a specific area is sufficiently refined and documented so that the activities in such area now have the potential to achieve their desired outcomes.

However, B-ITa in collaborative networked organizations (CNOs) has hardly been studied. Yet, this is important because improved B-ITa entails a more efficient use of IT in the CNO supporting the integration of information systems and processes across organizational boundaries. CNOs form the core of a new discipline that focuses on the structure, behavior, and dynamics of networks of independent organizations that collaborate using IT to better achieve common goals.

Notwithstanding the effective application of current B-ITa MMs for single organizations, to the best of our knowledge at the time of writing this dissertation, there is no MM that specifically addresses the processes needed for achieving alignment between business and IT in CNOs. In response, this dissertation introduces the ICoNOs MM, a MM to assess B-ITa in CNOs. The ICoNOs MM presents a roll up of recommendations - e.g., coordination mechanisms, implementation strategies and organizational changes, in the form of process areas, specific goals and practices. Through its maturity levels, the ICoNOs MM provides improvement routes for those domains that are the most important for achieving alignment in CNOs. We believe that achieving B-ITa in CNOs is more complex than in single organizations because in collaborative settings, B-ITa is driven by goals of different independent organizations commonly with no centralized decision-making processes.

Throughout this dissertation, we present the results of four literature surveys, one focus group, and six case studies. Based on these conceptual and empirical research activities, we designed and validated the components underlying the ICoNOs MM and the model itself. 



\section{Acknowledgements}

Over the last few years I have been tramping a research path, hoping to reach a milestone from which to look back and realize that I had made it. Now I am there. It was truly a great experience. Writing a dissertation can be a frustrating and lonely endeavor or, it can be an inspiring and intellectually stimulating joint venture. I have experienced both. The truth is that I would not have been able to complete successfully this $\mathrm{PhD}$ without the encouraging people around me. In an attempt to make up at least a bit to all these people, I will here try to mention some of you (although I know that such a list can never be complete and that I will most likely spend days blaming me for having missed some of the most important people out). Here we go:

Gert, my partner and best friend. I have not, cannot, and will never understand how you manage to put up with me and my way of being. I thank you deeply for all your love and for always being there!

Pascal van Eck and Maya Daneva, my supervisors. Without your competence, encouragement and endless support, this dissertation would never have been written. This is actually due to you!

Roel Wieringa, my promotor. Thanks goes out to you for your insightful suggestions, advice, and support that assisted me during the last stage of writing my dissertation.

I also want to thank

...all my colleagues at University of Twente who have suffered through my seminar presentations and given valuable feedback throughout my research. Specially, I thank Jelena Marincic, Novica Zarvić and Virginia Franqueira for their fellowship and support in the initial, middle and final phases of my $\mathrm{PhD}$, respectively; and for their friendship.

...all the people I had the opportunity to work with in different empirical settings. I would specifically like to mention Leida van Oene, Nicte-Há Castro Cárdenas, and Frank Snels. Thanks for making the work pleasant and valuable.

...all the anonymous reviewers of the papers upon which this dissertation is based, and all the active participants at the conferences where I have presented my ideas. Your questions, comments and suggestions have been most useful.

The few lines here at the end of the acknowledgements cannot show enough appreciation for the love and support I have received during my $\mathrm{PhD}$ time from my parents, Juan Roberto and Martha Elvira, my siblings, Juan Roberto and Martha Elvira, my (Mexican and Dutch) relatives, and my (Mexican and Dutch) friends. You have given me the opportunity to think about matters other than my dissertation, and by doing so you have always (implicitly) given me more strength to continue. 



\section{Contents}

$\begin{array}{ll}\text { Abstract } & \text { i }\end{array}$

$\begin{array}{ll}\text { Acknowledgements } & \text { iii }\end{array}$

List of figures $\quad$ xii

List of tables $\quad$ xiii

List of abbreviations $\quad$ xv

I Background 1

1 Introduction 3

1.1 Research motivation .................. 3

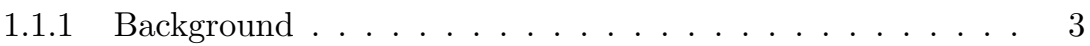

1.1.1.1 What is business-IT alignment? . . . . . . . . 3

1.1.1.2 What is a collaborative networked organization? . 4

1.1.1.3 What is a maturity model? . . . . . . . . . 5

1.1.2 The need for a new MM . . . . . . . . . . . . 5

1.2 Problem statement . . . . . . . . . . . . . . . 6

1.2.1 Scope of the model . . . . . . . . . . . . . 7

1.2.2 Research questions .................. 9

1.3 Scope of the research . . . . . . . . . . . . . . . . . . 11

1.4 Methodology at a glance . . . . . . . . . . . . . . . . . . 14

1.5 Contribution to knowledge . . . . . . . . . . . . . . . 15

1.6 Implications for practice . . . . . . . . . . . . . . . 16

1.7 Outline of the dissertation . . . . . . . . . . . . 17

2 Definitional foundations $\quad 19$

2.1 Business-IT alignment . . . . . . . . . . . . . . . . . . 19

2.1.1 A historical viewpoint of B-ITa . . . . . . . . . . 20

2.1.2 B-ITa perspectives ............... . 21

2.1.2.1 B-ITa as a strategic matter . . . . . . . . 21

2.1.2.2 B-ITa as a steady state ........... . . 23

2.1.2.3 B-ITa as a continuous process . . . . . . . . 23

2.1.2.4 B-ITa as a performance indicator . . . . . . . . . 23

2.1.2.5 B-ITa as a change driver . . . . . . . . . . 23

2.1.2.6 B-ITa as a social dimension . . . . . . . . . . . 24

2.1.2.7 B-ITa as a operational issue . . . . . . . . . 24

2.1.3 Definition of B-ITa . . . . . . . . . . . . . . . 24

2.2 Collaborative networked organizations . . . . . . . . . . . 27

2.2.1 The origin of CNOs . . . . . . . . . . . 28 
2.2.1.1 Transaction cost theory . . . . . . . . . . . 28

2.2.1.2 Resource dependence theory . . . . . . . . . 30

2.2.1.3 Information technology impact . . . . . . . . . 31

2.2.2 CNO-related terms . . . . . . . . . . . . . . 32

2.2.3 Definition of $\mathrm{CNO} \ldots \ldots . \ldots . \ldots 35$

2.3 Maturity models . . . . . . . . . . . . . . . . . . . . . . . . . . . . . . 38

2.3.1 The CMMI . . . . . . . . . . . . . . . . . 39

2.4 Summary ............................ 41

3 Methodology 43

3.1 Type of research ................... . . . 44

3.2 Research approach ................... . . . . . . . . . . . . . . . 45

3.2.1 Research methods . . . . . . . . . . . . . . . 47

3.2.1.1 Literature review . . . . . . . . . . . . . . 47

3.2.1.2 Focus group . . . . . . . . . . . . . . . 48

3.2.1.3 Case study . . . . . . . . . . . . . . . 49

3.2.1.4 Industrial trial/pilot assessment . . . . . . . 50

3.2.2 Data-gathering techniques . . . . . . . . . . . . 50

3.2.2.1 Semi-structured interviews .......... . 50

3.2.2.2 Observation ............... . . 51

3.2.2.3 Documents analysis ................. 51

3.2.3 Data analysis techniques .............. 51

3.2.3.1 Interpretation ............... . . 52

3.2.3.2 Hermeneutics . . . . . . . . . . . . . . 52

3.2.3.3 Realist approach ................ 53

3.3 Validity of the research . . . . . . . . . . . . . . . 53

3.3.1 Construct validity . . . . . . . . . . . . . 53

3.3.2 Internal validity . . . . . . . . . . . . . . . . . 55

3.3.3 External validity . . . . . . . . . . . . . . . 57

3.4 Summary .................... . . 58

4 Related work $\quad 61$

4.1 Assessment approaches of B-ITa . . . . . . . . . . . . . 62

4.1.1 Luftman's MM . . . . . . . . . . . . . . . . 62

4.1.2 CIO Council's assessment guide . . . . . . . . . . . 63

4.1 .3 Duffy's MM . . . . . . . . . . . . . . . . . 64

4.1.4 de Koning et al.'s model . . . . . . . . . . . . . . . . . . . 65

4.1.5 van der Raadt et al.'s MAAM . . . . . . . . . . . . 66

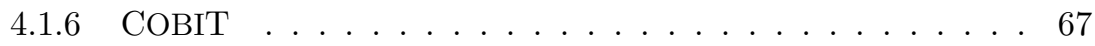

4.1.7 van der Zee et al.'s assessment tool . . . . . . . . . . . . 69

4.1.8 Sanchez Ortiz's B-ITa instrument . . . . . . . . . . . . 70

4.2 Comparative analysis of the models . . . . . . . . . . . . . . 70

4.2.1 MM development process ............... 71

4.2.2 B-ITa MMs comparison ... . . . . . . . . . 73 4.2.2.1 Type of the model: Assessment vs. development . 74 
4.2.2.2 Architecture of the model: Staged vs. continuous 74

4.2.2.3 Structure of the model . . . . . . . . . . 75

4.2.2.4 Levels in the model . . . . . . . . . . . . 75

4.2.2.5 Domains in the model . . . . . . . . . . . 77

4.2.2.6 Process areas in the model . . . . . . . . . . 77

4.2.2.7 Alignment level and orientation of the model . . . 77

4.3 Maturity models for CNOs . . . . . . . . . . . . . 78

4.3.1 Discussion on the observations . . . . . . . . . . . 81

4.4 Summary ....................... . . 82

$\begin{array}{lll}\text { II Solution } & 83\end{array}$

5 B-ITa domains: The initial attempts $\quad 85$

5.1 Introduction . . . . . . . . . . . . . . . . . . 85

5.2 B-ITa domains and their validation . . . . . . . . . . . . . 86

5.3 The validation process .................. 87

5.4 Focus group session . . . . . . . . . . . . . . . . . 90

5.4.1 Results ..................... 91

5.4 .2 Focus group validity threats . . . . . . . . . . . . . 92

5.5 Case study 1: Technology outsourcing relation . . . . . . . . . . 93

5.5.1 Research site .................. 93

5.5.2 Data sources and findings . . . . . . . . . . . . . . . 94

5.5.3 Case study validity threats . . . . . . . . . . . . 96

5.6 Implications of the results for the B-ITa domains . . . . . . . . . . 96

5.7 Summary ...................... . . . 99

6 B-ITa domains: Empirical validation 101

6.1 Introduction . . . . . . . . . . . . . . . . . 101

6.2 Multiple case study research approach . . . . . . . . . . . . . 102

6.3 Case study 2: State government collaboration . . . . . . . . . 105

6.3.1 Research design and findings . . . . . . . . . . . . . 107

6.3.2 Discussion . . . . . . . . . . . . . . . 110

6.4 Case study 3: Regional government network . . . . . . . . . . . . . 112

6.4.1 Research design and findings . . . . . . . . . . . . 113

6.4.2 Discussion . . . . . . . . . . . . . . 116

6.5 Case study 4: Provincial government relation . . . . . . . . . 116

6.5.1 Research design and findings . . . . . . . . . . . . . 118

6.5.2 Discussion . . . . . . . . . . . . . . . 121

6.6 Cross-case analysis . . . . . . . . . . . . . . . . . 122

6.6.1 Solution: the B-ITa domains . . . . . . . . . . 123

6.6.1.1 Partnering structure . . . . . . . . . . . 123

6.6.1.2 IS architecture and process architecture . . . . . 123

6.6.1.3 Coordination ................ 124

6.6.2 Mechanisms ................... 124 
6.6.2.1 Relations between the B-ITa domains . . . . . . . 124

6.6.2.2 Additional mechanisms . . . . . . . . . . . . . 126

6.6 .3 Context . . . . . . . . . . . . . . . . 126

6.7 Summary . . . . . . . . . . . . . . . . . . 127

7 B-ITa processes $\quad 129$

7.1 Introduction . . . . . . . . . . . . . . . . . . . . . . . 129

7.2 The ICoNOs MM: The first version . . . . . . . . . . . . . . . . . 129

7.2.1 First set of B-ITa process areas . . . . . . . . . . . . . 131

7.2.1.1 Partnering structure . . . . . . . . . . . . . 131

7.2.1.2 IS architecture . . . . . . . . . . . . . . . . . 131

7.2.1.3 Process architecture . . . . . . . . . . . . 132

7.2.1.4 Coordination . . . . . . . . . . . . . . 133

7.3 Literature survey . . . . . . . . . . . . . . . . . . . . . . . 134

7.3.1 Theories of B-ITa related constructs . . . . . . . . . 136

7.3.1.1 Gunderson's theory . . . . . . . . . . . . . 136

7.3.1.2 Hoque's theory . . . . . . . . . . . . . . . . . 137

7.3.1.3 Bodenstaff et al.'s theory . . . . . . . . . . . . 137

7.3.1.4 Ross' theory . . . . . . . . . . . . . . . . . 137

7.3.1.5 Chan's theory . . . . . . . . . . . . . 138

7.3.1.6 Holden and O'Toole's theory . . . . . . . . . . . . 138

7.3.1.7 Blue-Crow's theory . . . . . . . . . . . . . . 139

7.3.1.8 Mintzberg's theory . . . . . . . . . . . . . . . . 139

7.3.1.9 Decker and Lesser's theory . . . . . . . . . . . . . 140

7.3.2 Models covering B-ITa related constructs . . . . . . . . . 140

7.3.2.1 MetaGroup's model . . . . . . . . . . . . . . . 140

7.3 .2 .2 DYA . . . . . . . . . . . . . . . 141

7.3 .2 .3 TOGAF . . . . . . . . . . . 141

7.4 Case study 5: Regional government network . . . . . . . . . . . . 142

7.4.1 Data collection and analysis process . . . . . . . . . . 143

7.4 .2 Case study findings . . . . . . . . . . . . . . . . . . 145

7.4.2.1 Partnering structure . . . . . . . . . . . . . . . 146

7.4 .2 .2 IS architecture . . . . . . . . . . . . . . . . 149

7.4.2.3 Process architecture . . . . . . . . . . . . . 152

7.4.2.4 Coordination . . . . . . . . . . . . . . . . 154

7.4.3 Final statements . . . . . . . . . . . . . . . . 156

7.4.3.1 General conclusions . . . . . . . . . . . . . 156

7.4.3.2 B-ITa best practices . . . . . . . . . . 156

7.4 .4 Validity threats . . . . . . . . . . . . . . 158

7.4.4.1 Construct validity . . . . . . . . . . . . . . . . . 158

7.4 .4 .2 Internal validity . . . . . . . . . . . . . . 158

7.4.4.3 External validity . . . . . . . . . . . . . . 158

7.5 Summary . . . . . . . . . . . . . . . . . . . 159 
8 The ICoNOs MM 161

8.1 Partnering structure . . . . . . . . . . . . . . . 162

8.1.1 Business model definition . . . . . . . . . . . . . . 162

8.1.2 Governance structure and compliance . . . . . . . . . 165

8.1.3 Service level agreements definition . . . . . . . . . . 168

8.2 IS architecture . . . . . . . . . . . . . . . . 170

8.2.1 Current IS architecture description . . . . . . . . . . . 170

8.2 .2 IS capabilities definition . . . . . . . . . . . . . . . 171

8.2 .3 IS portfolio management . . . . . . . . . . . . . . . . . 172

8.2.4 IS requirements management . . . . . . . . . . . . . 175

8.3 Process architecture . . . . . . . . . . . . . . . . 177

8.3.1 Current process architecture description . . . . . . . . 177

8.3.2 Organizational process focus planning . . . . . . . . 178

8.3.3 Target process architecture formulation . . . . . . . . . 181

8.4 Coordination . . . . . . . . . . . . . . . . . 182

8.4.1 Communication-oriented coordination . . . . . . . . 182

8.4 .2 Direct supervision . . . . . . . . . . . . . . . . 184

8.4.3 Informal communication adjustment . . . . . . . . 185

8.4 .4 Standardization . . . . . . . . . . . . . . 187

$\begin{array}{ll}\text { III Validation } & 191\end{array}$

9 Pilot Assessment 193

9.1 Introduction . . . . . . . . . . . . . . . . . . . . 193

9.2 ICoNOs MM-assessed CNO . . . . . . . . . . . . . . . . . 194

9.3 Assessment procedure . . . . . . . . . . . . . . . . . . . 195

9.3.1 Data collection . . . . . . . . . . . . . . . . . 196

9.3 .2 Analysis process . . . . . . . . . . . . . . . . . . 197

9.4 Results and recommendations . . . . . . . . . . . . . . 198

9.4.1 Partnering structure . . . . . . . . . . . . . . . . 200

9.4 .2 IS architecture . . . . . . . . . . . . . . . . 203

9.4 .3 Process architecture . . . . . . . . . . . . . . 205

9.4 .4 Coordination . . . . . . . . . . . . . . . . . 208

9.4.5 Recommendations . . . . . . . . . . . . . . . . . 210

9.5 Usability of the ICoNOs MM . . . . . . . . . . . . . . 212

9.5.1 Perception-based evaluation approach . . . . . . . . . 213

9.5.2 Evaluation results . . . . . . . . . . . . . . . 2215

9.5.2.1 Perceived ease of use . . . . . . . . . . . 2 215

9.5.2.2 Perceived usefulness . . . . . . . . . . . . . 216

9.5.2.3 Intention to use . . . . . . . . . . . . . . 217

9.6 Limitations of the assessment . . . . . . . . . . . . . . . 218

9.7 Summary . . . . . . . . . . . . . . . . 218 
$\begin{array}{ll}\text { IV Conclusion } & 221\end{array}$

10 Discussion and future work 223

10.1 Reviewing the research questions . . . . . . . . . . . 224

10.2 Contributions . . . . . . . . . . . . . . . . 227

10.3 Limitations and open issues . . . . . . . . . . . . . . . . 228

10.3.1 ICoNOs MM shortcomings . . . . . . . . . . . . . . 229

10.3.1.1 Public vs. private CNOs . . . . . . . . . . . . . 229

10.3.1.2 ICoNOs MM validation . . . . . . . . . . . . . 229

10.3.1.3 Model assessment tool . . . . . . . . . . . . . . 229

10.3.2 Open issues . . . . . . . . . . . . . . . . . . 230

10.4 Future work . . . . . . . . . . . . . . . . . . 230

10.4.1 Work based on the limitations and open issues . . . . . 230

10.4.2 Work based on the B-ITa research framework . . . . . . . . 231

10.4.3 New hypotheses for a research agenda on B-ITa . . . . . . . 231

Appendices $\quad 235$

$\begin{array}{ll}\text { A Articles upon this dissertation is based } & 237\end{array}$

B Generic goals and practices in the CMMI 239

C Questionnaire used in the case study 5

$\begin{array}{ll}\text { D Pilot assessment questions } & 243\end{array}$

$\begin{array}{ll}\text { E Answers to the post-task survey } & 247\end{array}$

$\begin{array}{ll}\text { Bibliography } & 251\end{array}$

$\begin{array}{ll}\text { Samenvatting } & 271\end{array}$

$\begin{array}{ll}\text { Resumen } & 273\end{array}$

$\begin{array}{ll}\text { SIKS Dissertation Series } & 275\end{array}$ 


\section{List of Figures}

1.1 Business-IT alignment framework in CNOs. . . . . . . . . . . . 4

1.2 Scope of the model: An illustrative example. . . . . . . . . . . 7

1.3 Research plan of this study. . . . . . . . . . . . . . . . . . . 10

1.4 Research methodology. . . . . . . . . . . . . . . . . . . . . . 14

1.5 The continuous architecture of the model: An illustrative example. 16

1.6 Layout of the dissertation. . . . . . . . . . . . . . . . . . 17

2.1 The evolution of B-ITa (adapted from [138]). . . . . . . . . 20

2.2 The B-ITa concept: A high level view. . . . . . . . . . . . 26

2.3 The levels in the CMMI. . . . . . . . . . . . . . . . . 40

3.1 Research methodology (taken from Chapter 1). . . . . . . . . . 46

3.2 Strategies used to promote validity in our research. . . . . . . . . . 54

4.1 MM development process. . . . . . . . . . . . . . . . . 71

4.2 Structure of the ICoNOs MM. . . . . . . . . . . . . . 76

4.3 The B-ITa levels in the ICoNOs MM. . . . . . . . . . . . 77

5.1 First proposal of the B-ITa domains. . . . . . . . . . . . 86

5.2 B-ITa domains validation approach. $\ldots \ldots \ldots \ldots$

5.3 The 'new' domains of the model. . . . . . . . . . . . . . . 97

6.1 Multiple case study research approach. . . . . . . . . . . . . 103

6.2 A research cycle for theory building (adapted from $[230,150]$ ). . . 104

6.3 Initial CSMO configuration. . . . . . . . . . . . . . . . . . 104

6.4 IT and business sides in the Tamaulipas government structure. . . 106

6.5 First refinement of the CSMO configuration. . . . . . . . . . . . 111

6.6 The To-Be state of the Overijssel CNO: Functional view. . . . . . . 113

6.7 Second refinement of the CSMO configuration. . . . . . . . . . 117

6.8 Relations between the B-ITa domains in CNOs. . . . . . . . . . . 125

7.1 ICoNOs MM: The first version. . . . . . . . . . . . . . . 130

7.2 Map modeling theories applicable to the B-ITa domains. . . . . . . 135

7.3 The data analysis process. . . . . . . . . . . . . . 145

7.4 B-ITa process areas performed in the Overijssel CNO. . . . . . . 146

7.5 The Overijssel CNO: Future situation (adapted from [139, 245, 323]).147

7.6 Overview of the ISs As-Is state in the province of Overijssel. . . . . 150

7.7 General processes supported by ISs in the Overijssel CNO. . . . . . 151

7.8 The ISs To-Be state in the Overijssel CNO: An example. . . . . . . 151

7.9 To-Be state of one collaborative process in the Overijssel CNO. . . 153

8.1 The ICoNOs MM. . . . . . . . . . . . . . . . . . . . 162 


\section{LIST OF FIGURES}

9.1 The 3TU.Federatie at a glance. . . . . . . . . . . . . . . . 195

9.2 Continuous consolidation analysis process. . . . . . . . . . . . 197

9.3 IS architecture of the Eindhoven University of Technology [87]. . . 203

9.4 The Method Evaluation Model (adapted from [209]). . . . . . . . . 213

10.1 MM development process revisited (adapted from Figure 4.1). . . . 225

10.2 The ICoNOs MM at a glance (taken from Chapter 8). . . . . . . . 227

10.3 Claim derived from the presented research (adapted from Figure 6.8).232 


\section{List of Tables}

1.1 Research framework for B-ITa (based on Thomas \& DeWitt in [188]) 11

1.2 Research methods. . . . . . . . . . . . . . . . . . 15

$2.1 \quad$ B-ITa definitions. . . . . . . . . . . . . . . . . . . 22

2.2 Matrix concept-indicator: The summary of findings. . . . . . . . . 35

2.3 Cross-organizational structures and their principal components. . . 36

3.1 Research methods (taken from Chapter 1). . . . . . . . . . . . 47

4.1 Comparative analysis of B-ITa assessment approaches. . . . . . . . 73

5.1 Distribution of participants' expertise in the focus group session. . 90

6.1 Educative credit request process. . . . . . . . . . . . . . . 110

6.2 Documents studied in the Ontario CNO case. . . . . . . . . . . . 119

7.1 Distribution of interviewees' expertise. . . . . . . . . . . . . . 144

9.1 Pilot assessment results: The 3TU.Federatie B-ITa maturity. . . . 199

9.2 Post-task survey for the perception-based evaluation. . . . . . . . . 214

10.1 Research activities, contributions and publications. . . . . . . 228 



\title{
List of Abbreviations
}

\author{
ACQ Acquisition \\ ATF IS Architecture Target Formulation \\ ATHENA Advanced Technologies for interoperability of Heterogeneous \\ Enterprise Networks and their Applications \\ AVV IS Architecture Verification and Validation \\ BAD Baseline IS Architecture Description \\ B-ITa Business-IT alignment \\ BITAMA B-ITa Maturity Audit tool \\ BMD Business Model Definition \\ BPD Baseline Process architecture Description \\ CASS Citizen Attention Service System \\ CAR Causal Analysis and Resolution \\ CEO Chief Executive Officer \\ CFO Chief Financial Officer \\ CGP Cross-Governmental Partnerschip \\ CIC Citizenship and Immigration Canada \\ CIO Chief Information Officer \\ CMMI Capability Maturity Model Integration \\ CMO Context-Mechanism-Outcome \\ $\mathrm{CNO} \quad$ Collaborative Networked Organization \\ $\mathrm{CO} \quad$ Coordination \\ COBIT Control Objectives for Information and related Technology \\ COC Communication-Oriented Coordination \\ CollabMM Collaboration Maturity Model \\ CPD Current Process architecture Description \\ CSA Current IS Architecture description \\ CSMO Context-Solution-Mechanism-Outcome \\ DEV Development \\ DTS Direct Supervision \\ DYA Dynamic Architecture \\ EA Enterprise Architecture \\ E2aMM Extended Enterprise Architecture Maturity Model \\ EDOC Enterprise Distributed Object Computing \\ EFC Event logs Formal Consistency \\ EIMM Enterprise Interoperability Maturity Model \\ ESB Enterprise Service Bus \\ GSC Governance Structure and Compliance \\ GUI Graphical User Interface \\ ICoNOs MM IT-enabled Collaborative Networked Organizations MM \\ $\mathrm{ICT}$ \\ $\mathrm{InCA}$ \\ Information and Communication Technology \\ IOS Inter-organizational Systems \\ IoAO Inter-organizational IS Architecture Optimization
}




\section{LIST OF ABBREVIATIONS}

$\begin{array}{ll}\text { IoPD } & \text { Inter-organizational Policies Definition } \\ \text { IoPO } & \text { Inter-organizational Process Optimization } \\ \text { IS } & \text { Information System } \\ \text { IsCD } & \text { IS Capabilities Definition } \\ \text { IsPM } & \text { IS Portfolio Management } \\ \text { IsRM } & \text { IS Requirements Management } \\ \text { IT } & \text { Information Technology } \\ \text { KPI } & \text { Key Performance Indicator } \\ \text { LINC } & \text { Language Instruction for Newcomers to Canada } \\ \text { LVO } & \text { Landelijke Voorziening Omgevingsloket } \\ & \text { (National online all-in-one service for environmental permits) } \\ \text { MAAM } & \text { Multi-dimensional Assessment model for architecture } \\ & \text { Alignment and Maturity } \\ \text { MBNQA } & \text { Malcolm Baldrige National Quality Award } \\ \text { MCI } & \text { Ministry of Citizenship and Immigration } \\ \text { MM } & \text { Maturity Model } \\ \text { MRE } & \text { Metric-based Roles Exploration } \\ \text { NORA } & \text { Nederlandse Overheid Referentie Architectuur } \\ & \text { (Dutch government reference architecture) } \\ \text { OPP } & \text { Organizational Process Performance } \\ \text { OSD } & \text { Organizational Structure Definition } \\ \text { OWD } & \text { Ontario's Women's Directorate } \\ \text { PA } & \text { Process Architecture } \\ \text { PAD } & \text { Process Architecture Definition } \\ \text { PAF } & \text { Process Architecture target Formulation } \\ \text { PFP } & \text { Organizational Process Focus Planning } \\ \text { PPM } & \text { Process Portfolio Management } \\ \text { PS } & \text { Partnering Structure } \\ \text { QPM } & \text { Quantitative IS Portfolio Management } \\ \text { QRA } & \text { Quantitative coordination Relation Analysis } \\ \text { RAM } & \text { Risk Analysis and Mitigation } \\ \text { RDT } & \text { Resource Dependence Theory } \\ \text { ROI } & \text { Return On Investment } \\ \text { RRS } & \text { Roles and Responsibilities Specification } \\ \text { SAM } & \text { Strategic Alignment Model } \\ \text { SCAMPI } & \text { Standard CMMI Appraisal Method for Process Improvement } \\ \text { SCM } & \text { Supply Chain Management } \\ \text { SEI } & \text { Software Engineering Institute } \\ \text { SIF } & \text { Shared Interoperability Framework } \\ \text { SLA } & \text { Service Level Agreement } \\ \text { SOA } & \text { Service Oriented Architecture } \\ \text { SPD } & \text { Standards and Principles Definition } \\ \text { SSCAN } & \text { Settlement Sector Client Administration Network } \\ \text { STD } & \text { Standardization } \\ \text { SVC } & \text { Services } \\ & \end{array}$


SW CMM Software Capability Maturity Model

TOGAF The Open Group Architecture Framework

TPA Target Process Architecture formulation

TCT Transaction Costs Theory

VROM Volkshuisvesting, Ruimtelijke Ordening en Milieubeheer

(Housing, spatial planning and environmental management)

WABO Wet Algemene Bepalingen Omgevingsrecht

(Enviromental licensing - general provisions - bill) 



\section{Part I}

\section{Background}





\section{1 \\ Introduction}

This chapter provides an overview of the research published in this dissertation. It presents the background of the investigated problem, the drivers of the research, the research statement, and the research questions. Furthermore, it establishes the scope and the methodology of the research and states the contributions to knowledge of the presented work and the implications for practice. The chapter concludes with an outline of the dissertation.

\subsection{Research motivation}

\subsubsection{Background}

In this section, we outline our definitions and assumptions concerning business-IT alignment (B-ITa), collaborative networked organizations (CNOs), and maturity models (MMs). This serves as definitional basis for the rest of the dissertation. Detailed analysis of these concepts is presented in Chapter 2.

\subsubsection{What is business-IT alignment?}

There are several definitions of B-ITa that can be found in literature (see Section 2.1 in Chapter 2). For the purpose of this dissertation, we define B-ITa in CNO settings as the process to make IT services support the requirements of the business, whether such services are individually or collaboratively offered.

In this definition, we do not consider alignment as a steady state but as a process that needs to be performed continuously - this will be discussed in greater detail in Chapter 2. By 'IT services' we mean services offered by computerized information systems (ISs). By the 'requirements of the business' we mean those systems requirements derived from analyzing the goal(s) and the processes of the $\mathrm{CNO}$, and the relations between the participating organizations. We will focus on operational B-ITa, which consists of aligning the operational activities of IT systems and people to each other so that optimal IT support for business goals is achieved. This contrasts with strategic B-ITa, where business and IT goals and

policies are decided, without elaborating operational details [50, 189, 253] (see Chapter 2). 


\section{CHAPTER 1. INTRODUCTION}

We analyze the B-ITa concept in CNOs based on the scheme shown in Figure 1.1. The horizontal layers classify entities in a service provisioning hierarchy in a business: physical entities provide services to a software infrastructure, which provides services to ISs, which provide services to businesses. In the business layer, we take four views on businesses: businesses provide services that have a u-

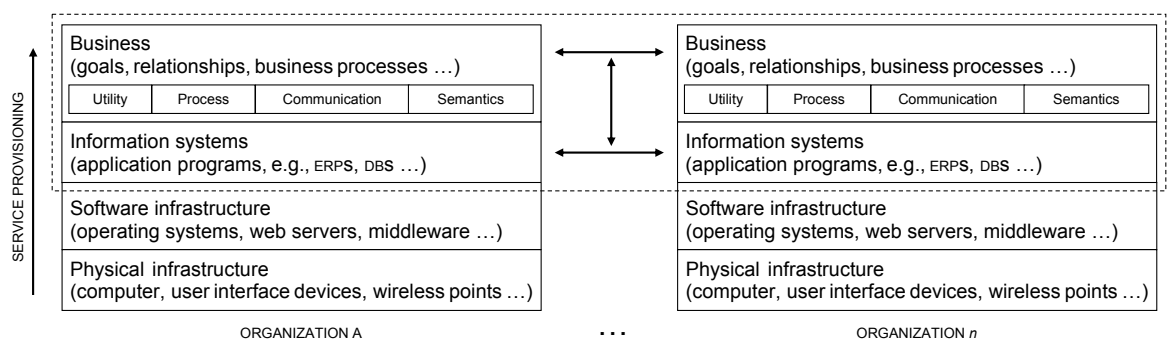

Figure 1.1: Business-IT alignment framework in CNOs.

tility, they perform processes to provide these services, they communicate with one another as part of performing these processes, and while doing that, they exchange data that has semantics. Participating organizations in a CNO need both to fit the different entities (horizontal arrows) as well as to address B-ITa (vertical arrow). Our interest is in the upper two layers of the framework (area delimited by the dotted line), because there is where business and IT alignment in CNOs takes place.

\subsubsection{What is a collaborative networked organization?}

We define a CNO to be any "mix-and-match" network of profit-and-loss responsible organizational units, or of independent organizations, connected by IT, that work together to jointly accomplish tasks, reach common goals and serve customers over a period of time [267]. Virtual enterprises [12], value constellations [298], extended enterprises and collaborative highly integrated supply chains [76] are some forms of CNOs. Our interest is in IT-enabled CNOs, i.e., collaborations that are made possible by IT where the participants interoperate with each other by means of ISs. We believe that IT makes global competition and collaboration possible, forcing organizations to focus on what they can do well and facilitating collaboration between organizations with complementary competencies.

Modern business environments are becoming more competitive, often due to global commercial pressure. This change in the business environment forces organizations to re-think the way they are doing business. More and more organizations nowadays take advantage of the next level of reengineering approaches which capitalize on connecting and aligning the business and IT operations of one organization with those of other organizations to meet common organizational goals. These cross-organizational connections lead up CNOs. There are 


\subsection{RESEARCH MOTIVATION}

furthermore several theories to explain the origin of CNOs. We review these theories in Chapter 2.

\subsubsection{What is a maturity model?}

MMs describe the evolution of a specific entity over time. Commonly, this entity is an organizational area or function. MMs have been developed to assess specific areas against a norm. Based on maturity assessments, organizations know the extent to which activities in such areas are predictable. That is, organizations can be aware of whether a specific area is sufficiently refined and documented so that the activities in this area now have the potential to achieve their desired outcomes. MMs apply a life-cycle approach where an area develops over time until it reaches its highest maturity level. This does not mean that such an area is always going to reach the highest level of maturity. An MM shows the way to reach that level. Organizations can then decide what maturity level is the level that better meets their goals. For example, the well-known software capability maturity model ${ }^{1}$ (SW CMM) proposed by Carnegie Mellon University's Software Engineering Institute (SEI) identifies, specifically for software production, several levels of software process management sophistication. However, organizations that use the SW CMM determine themselves what level is the most appropriate target for the management of their software development projects.

Essentially, MMs make it easier for organizations to establish goals for process improvement and identify opportunities for optimization, since these models describe basic attributes that are expected to characterize a particular area for each maturity level. By comparing an organization's characteristics and attributes with an MM, an organization identifies how mature it is in order to increase its process capability: first, establishing goals for the improvement of processes and then, taking action to achieve them. In Section 2.3 of Chapter 2, we present more details regarding MMs.

\subsubsection{The need for a new MM}

Aligning IT with the business remains one of the top priorities for both business practitioners and researchers. Interest in B-ITa is stimulated by cases of organizations that have successfully aligned their IT to gain competitive advantage $[154,241]$ and to improve organizational performance $[108,262]$.

In organizations of any significant size, B-ITa is a hard problem that requires continuous attention. Within the broad scope of literature on alignment, some authors have stressed the importance of assessing B-ITa in order to plan B-ITa improvement actions - e.g. [80, 95, 189, 312]. In support of this, those authors have developed MMs to assess the alignment between IT services and the requirements of the business in single organizations. However, the problem of B-ITa in CNOs has hardly been studied. Yet, the problem is important because improved B-ITa entails a more efficient use of IT in the CNO supporting the integration of

${ }^{1}$ More information on http://www.sei.cmu.edu/cmm/ 
enterprise applications and processes across organizational boundaries [85, 352]. We believe that achieving B-ITa in CNOs is more complex than in single organizations because in such settings, B-ITa is driven by economic processes instead of by centralized decision-making processes.

Notwithstanding the effective application of those MMs developed to assess B-ITa in single organizations, to the best of our knowledge there is no MM that specifically addresses the processes needed for achieving alignment between business and IT in CNOs. B-ITa in CNOs takes specific characteristics of crossorganizational settings into account, e.g., that different decisions are often taken at different times and by different individuals or groups. Clearly, some other models and theories (e.g., the informal organizational structure on B-ITa of Chan [48], the B-ITa effect in the new economy of Hoque [138], the coordination theory of Mintzberg [206], the extended enterprise architecture MM [280], the B-ITa MM of Duffy [95]) can also be used to understand aspects related to B-ITa in CNOs. Nevertheless, none of these models and theories covers all the necessary domains that need to be considered by CNOs when achieving B-ITa. They are oriented to single organizations and ignore characteristics specific of CNOs such as the need for coordination or structural and authoritarian relationships (see Chapter 4 where we introduce and compare several MMs). CNOs have to spend considerable time and effort to understand and apply different models, and to analyze how the results could combine to plan B-ITa improvement actions. This motivated us to adopt the position that these models and theories might be used as starting points in cross-organizational B-ITa initiatives. However, they need to be integrated. In response to this, we introduce in this dissertation an MM to assess B-ITa in CNOs: the ICoNOs MM.

\subsection{Problem statement}

In a CNO, each participating organization can have a different level of maturity in its alignment between business and IT. The maturity of each participating organization influences the maturity of B-ITa of the entire CNO. For example, suppose that a CNO is composed of organizations A, B and C. Organization A with maturity level 3 of B-ITa as single organization can compel organizations B and $\mathbf{C}$ to collaboratively address the B-ITa domains considered by organization $\mathbf{A}$ to achieve the same maturity level (i.e., maturity level 3) as a networked organization. One of the reasons that causes the real-life scenario presented in this example is the lack of a framework to guide CNOs in their efforts to achieve cross-organizational B-ITa. The identification of B-ITa domains and processes in a cross-organizational environment is hardly addressed at all. Nowadays, there is no MM to assess B-ITa in CNOs.

We are addressing this problem by developing a new MM: the so called ICoNOs MM (IT-enabled Collaborative Networked Organizations Maturity Model) to assess B-ITa in cross-organizational settings. In order to develop such a model, a number of challenging modeling and design decisions need to be made: 
- What are the problems with the current MMs?

- What are the improvement goals?

- What is the type and architecture of the model?

- How can the levels be defined?

- What are the domains to include in the model?

- Which processes relate to these domains?

- How can the model be validated?

Each of these decisions represents a major research challenge. Picking up these challenges, we define a number of research questions which guide the research presented in this dissertation. We present these research questions in Section 1.2.2. To this end, in the next section, we define the scope of the ICoNOs MM.

\subsubsection{Scope of the model}

Determining the scope of the model means to set the boundaries for the model's application and use, and to define the purpose of the model. This is to differentiate the model from existing MMs. As the ICoNOs MM will assess B-ITa in a CNO, it will be applied to determine the maturity of the B-ITa of the whole networked organization and not of the B-ITa of each individual participating organization. This concept is clarified in Figure 1.2 where we present an example of two configurations of a CNO composed of four organizations: $\mathbf{A}, \mathbf{B}, \mathbf{C}$, and $\mathbf{D}$. The dotted lines in this figure represent the binary relations between the different CNO participants. The (sets of) Greek letters group such binary relations from the particular point of view of each participating organization. For example, in a CNO mesh configuration (see on the left side of Figure 1.2), a B-ITa assessment of the set of $\beta$ 's will represent the B-ITa maturity of organization $\mathbf{B}$ with respect to organizations $\mathbf{A}, \mathbf{C}$ and $\mathbf{D}$. However, in a CNO star configuration (see on the right side of Figure 1.2), the same assessment will only stand for the B-ITa maturity of organization $\mathbf{B}$ with respect to organization $\mathbf{A}$.
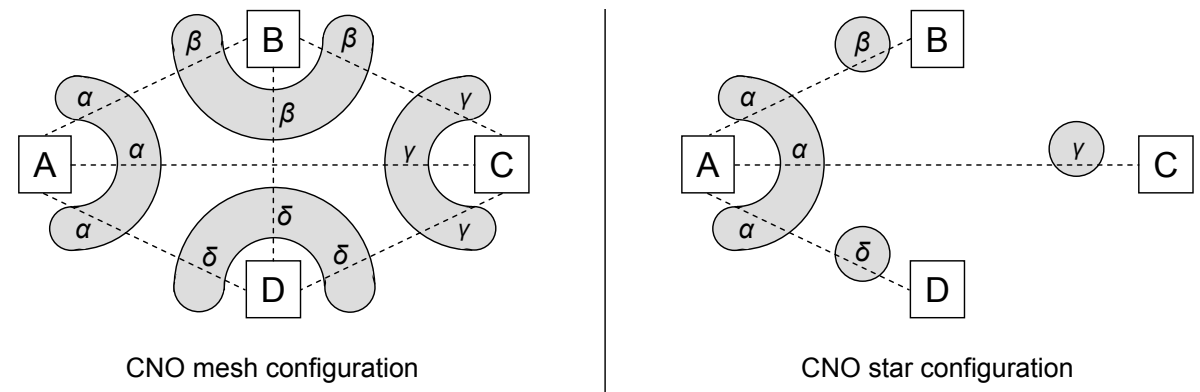

Figure 1.2: Scope of the model: An illustrative example.

Different B-ITa maturity levels can then be presented in a CNO. CNOs can have a B-ITa maturity level for (i) each relation between two of the participating 


\section{CHAPTER 1. INTRODUCTION}

organizations, (ii) each relation between one organization with respect to the other ones, (iii) each individual participating organization, and (iv) the entire CNO. These maturity levels can be set down by conducting four different types of B-ITa assessments:

1. a B-ITa assessment for each possible binary relation between the organizations in the CNO (see dotted lines in Figure 1.2). This implies that an assessment of this type will yield statements as "the maturity level of the B-ITa between organization A and organization B is X" or "the maturity level of the B-ITa between organization B and organization D is Y".

2. a B-ITa assessment for the relations between one organization and each of the other CNO participants. In Figure 1.2, from the perspective of organization $\mathbf{A}$, it will be the $\alpha$ 's; from the perspective of organization $\mathbf{B}$, it will be the $\beta$ 's; from the perspective of organization $\mathbf{C}$, it will be the $\gamma^{\prime}$ 's; and from the perspective of organization $\mathbf{D}$, it will be the $\delta$ 's.

3. a B-ITa assessment for each participating organization. Current MMs, which by the way are all focus on single organizations, can be used for this type of assessments (see Chapter 4). Their assessment results present statements as "the B-ITa maturity level in organization A is X".

4. a B-ITa assessment of the entire CNO that will result in a set of statements about how well a CNO is in its efforts for achieving B-ITa as a whole. It must be noted that this type of assessment is not the sum of all other assessment types. Instead, it indicates how well the entire CNO is in B-ITa exhibiting characteristics not recognizable from the individual perspectives of the participating organizations.

The ICoNOs MM addresses this last type of assessment considering the CNO as a whole. The model takes three special characteristics of CNOs into account, namely the need for coordination, the lack of centralized decision making and the heterogeneity of IS architectures. CNOs can adopt the ICoNOs MM to improve the processes needed for achieving cross-organizational B-ITa. However, the decisions concerning achieving, or assessing, B-ITa in a CNO can be made by one participating organization. We make the note that types 1 and 2 are also assessments of cross-organizational B-ITa, specifically of dyadic collaborations. The difference between these two types and type 4 is the perspective taken when conducting the assessment, i.e., dyadic perspective versus network perspective. If a dyadic collaboration is considered as a CNO, or if the goal of the B-ITa assessment is to assess B-ITa in one part of the CNO only, then the ICoNOs MM could be applied to assess such B-ITa. However, even though those assessments could be useful, a dyadic perspective is not considered in the development of the ICoNOs MM. The ICoNOs MM has been developed to assess B-ITa in CNOs from the perspective of the entire network. 


\subsection{PROBLEM STATEMENT}

\subsubsection{Research questions}

To discuss our research questions, we use the distinction between design research questions and knowledge research questions. According to Wieringa and Heerkens [337], design research questions ask for a way to achieve a desired output from a given input. They relate to a situation where some change needs to be enacted according to the way we think the world should be. This type of questions focus mainly on 'how-to-do' questions. The second type of research questions is the knowledge question. Knowledge research questions emerge when there is a difference between what we know about the world and what we would like to know. Within the context of these questions, we need to study the world to obtain knowledge related to a particular aspect. This type of questions usually focus on 'what-is-the-case' or 'why-is-it-the-case' questions. It must be noted that to solve a knowledge question conceptual modeling questions are required. These questions focus on constructing a system of concepts that can be used to design an artifact or to solve knowledge research questions. Figure 1.3 uses this distinction to decompose our research goal into research questions.

We start our research being interested in the built-in design knowledge of the current assessment approaches of B-ITa. Our particular interest lies in identifying and reusing this knowledge in future design so that we avoid developing a new approach from scratch which, in turn, makes the development process cheaper and faster. Most importantly, reusing also prevents future users from starting over when adopting the ICoNOs MM. This is accounted for in our first research question:

\section{Q1 What are the current assessment approaches of B-ITa?}

The purpose of this question is to identify advantages and shortcomings of current B-ITa assessment approaches in order to define the improvement goals and reusable knowledge. Next, we need to know:

\section{Q2 How to develop an improved approach (in our case an MM)?}

Considering the research challenges listed above in Section 1.2, we need to find a systematic approach to transform the design knowledge into an MM. Finally, after developing a new MM to assess B-ITa in CNOs, we should evaluate the model answering the next question:

\section{Q3 Is the improved approach valid and useful?}

For the purpose of this dissertation, we consider Q1, Q2 and Q3 as our main three research questions. These research questions guide the study presented in this dissertation. In Section 1.7 we present which research questions are addressed in which of the chapters. 
CHAPTER 1. INTRODUCTION

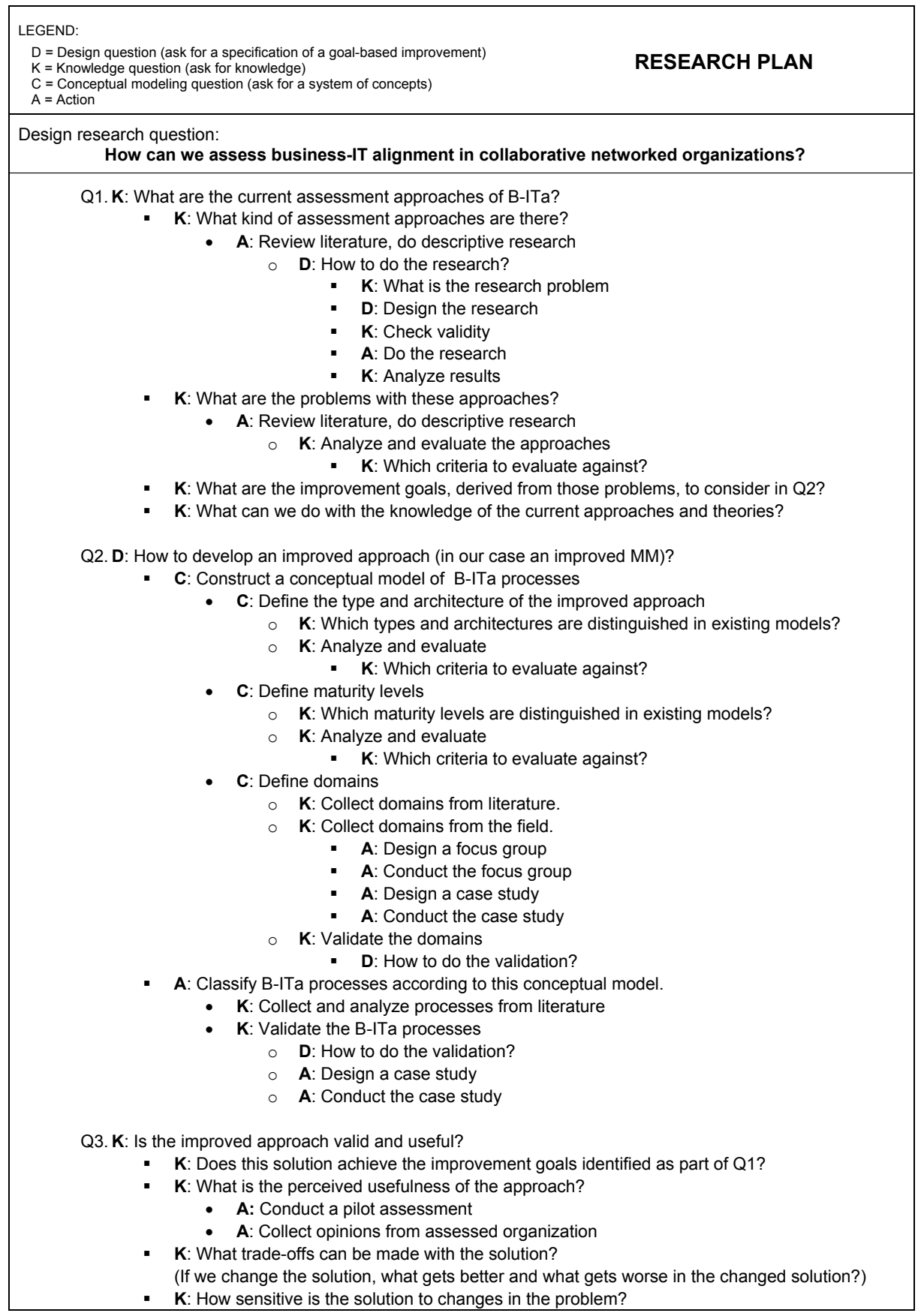

Figure 1.3: Research plan of this study. 


\subsection{Scope of the research}

The objective of this research is to enable assessing B-ITa in CNOs by developing the ICoNOs MM. This section positions our research in a research framework for alignment studies (see Table 1.1) in order to present explicitly its scope. This research framework for alignment studies is based on the framework presented by Luftman [188] who used the work of Snow and Thomas [290] to classify alignment studies according to the research approach the studies followed. In this framework, a research process is divided in two general themes: concept definition and concept validation. Each of these themes is decomposed in three phases: description, explanation and prediction [290].

We explicitly want to mention that because our research uses the realist theory-making approach of Pawson and Tilley [230] (see Chapter 3), we matched these description, explanation and prediction phases with the concepts involved in the main claim of the realist perspective: successful 'outcomes' can occur in organizations only if they introduce the appropriate ideas and opportunities ('mechanisms') in the appropriate 'contexts'. First, the description phase covers aspects related to the conceptual model that, according to Pawson and Tilley [230], needs to be established in order to define possible hypothesis to explore and to evaluate. Then, the explanation phase matches the mechanism concept of the realist perspective, i.e., both the explanation phase and the mechanism concept look at the precise manner in which the concepts identified in the description phase relate to produce a particular outcome. Finally, the context concept introduced by Pawson and Tilley [230] corresponds to the prediction phase because it refers to the circumstances into which a particular outcome takes place.

In the next paragraphs, we present details of each of the cells of this research framework for B-ITa.

Table 1.1: Research framework for B-ITa (based on Thomas \& DeWitt in [188])

\begin{tabular}{|c|c|c|c|}
\hline & Description & Mechanism & Context \\
\hline 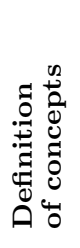 & $\begin{array}{l}\text { Research on concepts } \\
\text { and variables related } \\
\text { to alignment. } \\
\text { E.g. Henderson and } \\
\text { Verkatraman [130, 131], } \\
\text { Maes [192] }\end{array}$ & $\begin{array}{l}\text { Explanations } \\
\text { concerning how/why } \\
\text { such variables are } \\
\text { related. } \\
\text { E.g. Keen [156], } \\
\text { Henderson and } \\
\text { Thomas [129] }\end{array}$ & $\begin{array}{l}\text { Research to define in } \\
\text { which circumstances } \\
\text { those relations take } \\
\text { place. } \\
\text { E.g. Luftman et } \\
\text { al. [190], Broadbent } \\
\text { and Weill [30] }\end{array}$ \\
\hline 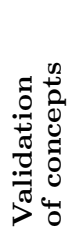 & $\begin{array}{l}\text { Test and validation of the } \\
\text { concepts and variables. } \\
\text { E.g. Chan and Huff [49], } \\
\text { Reich and Benbasat [253] }\end{array}$ & $\begin{array}{l}\text { Documentation of } \\
\text { previous researchs } \\
\text { using complex studies. } \\
\text { E.g. Thomas and } \\
\text { DeWitt [303], Chan et } \\
\text { al. [50], Croteau and } \\
\text { Bergeron [70] }\end{array}$ & $\begin{array}{l}\text { Test of explanations of } \\
\text { the relations between } \\
\text { alignment and other } \\
\text { topics. } \\
\text { E.g. Luftman et } \\
\text { al. [191], Poels [237] }\end{array}$ \\
\hline
\end{tabular}


The first theme, concept definition, covers the identification of the relevant concepts and variables related to alignment (description), the explanation of the relation between those concepts (mechanism), and the investigation of the environment where those relations take place (context). Each of these phases helps to build theory related to the alignment problem. Together, these three phases give new insights concerning what B-ITa is, how its components are related, and in which circumstances such alignment can happen. Explanation of these three phases is presented below.

\section{Description.}

The main goal of the description is to answer 'what' questions. The description plays a determining role during the progressive development of B-ITa concepts when identifying and refining the basic components of its theory and how they are related. The strategic alignment model (SAM), as it was originally described by Henderson and Verkatraman [130, 131], is perhaps the governing framework in the alignment research field. This model establishes the fundamental vocabulary and relations on which most of the succeeding work on alignment has been based. This conceptual model has demonstrated its success by several extensions and evolutions developed by other researchers (e.g., Maes [192]).

\section{Mechanism.}

The main goal of the mechanism phase is to determine 'how' and 'why' the concepts identified in the previous phase are related. It is an exploration of the nature and degree of the consistency between the different concepts. The so-called 'fusion map' described in the work of Keen [156], is a good example of a study in this phase. He states that an open dialogue between senior management and IT managers is required to be able to manage the relation between IT and business in the most efficient way possible in order to create a sustained competitive advantage. Another example is the work of Henderson and Thomas [129], who provide detailed explanations of the diverse relations of the concepts covered by SAM in the health care domain.

\section{Context.}

The main goal of the context phase is to determine the conditions in which the alignment theory takes place. Therefore, the questions that are addressed in this phase are 'who', 'where' and 'when'. A study in this phase incorporates the previously identified concepts and relations, and determines the limits in which they exist. For example, using the SAM, Luftman et al. [190] have found four perspectives that can lead to business transformation through IT. Another example is the study of Broadbent and Weill [30]. They sought for evidence of B-ITa in the use of IT in a case study conducted in four banks. They identified organizational practices that can influence the competitive advantage of organizations. 


\subsection{SCOPE OF THE RESEARCH}

The second theme, concept validation, also includes description, mechanism and context. The main difference with the definition of concepts lies in the use of deeper studies which go much beyond the use of a single case study.

\section{Description.}

The description in the validation of concepts covers testing and measuring the concepts identified in the concept definition. This phase of research has become important since the measures of concepts have been recognized as instrumental to B-ITa improvements. For example, Chan and Huff [49] have developed an instrument based on different theoretical perspectives to assess the link between business and IT. In a similar way, Reich and Benbasat [253] have measured the linkage between IT and business. Conducting a multiple case study in 10 business units at three major insurance companies, they also validated two proposed measures of alignment, namely understanding of current objectives and congruence in IT vision.

\section{Mechanism.}

The studies in this phase verify the relation of B-ITa concepts using statistical techniques. A good example is the research done by Thomas and DeWitt [303]. These authors collected data by using multiple methods and 134 top management teams in two American hospitals. They analyzed the relations between alignment and the results of the overall performance of those hospitals. Another example is the work done by Croteau and Bergeron [70]. On the basis of a case study in 223 organizations, they identified several profiles in the application of IT and specific business strategies that can promote effective performance. Similarly, using a mail survey, Chan et al. [50], found a relation between business/IT strategy and B-ITa, and its impact on business/IT performance.

\section{Context.}

The context phase in the validation of concepts aims at testing the explanations of the relations between alignment and other topics in order to predict what could happen in particular situations. For example, Luftman et al. [191] used a longitudinal study to look for enablers and inhibitors that help and hinder alignment. They analyzed the results considering the background of the organizations, the time and the position of the managers who took part in the study. Another example is the work of Poels [237]. After interviewing CEOs, CIOs and CFOs, he found 12 interventions that improve B-ITa and he demostrated a positive correlation between B-ITa and the return on IT investments.

The scope of our research covers the shaded cells in Table 1.1. To develop the ICoNOs MM (see Q2 in Figure 1.3), we need to identify the domains and processes

related to alignment in a cross-organizational environment. We, then, need to find evidence concerning how those domains and processes relate to B-ITa and 


\section{CHAPTER 1. INTRODUCTION}

in which particular settings such relations can be found. Test and validation of these domains and processes also is an important part of the study presented in this dissertation (see Q3 in Figure 1.3).

\subsection{Methodology at a glance}

This research is conceptual, qualitative and interdisciplinary. It is an investigation that involves synthesizing and integrating information in order to develop a final MM. A high-level description of the approach we adopt for this research is presented in Figure 1.4. It shows that our research comprises three main phases: (1) problem investigation, (2) solution design, and (3) solution validation.

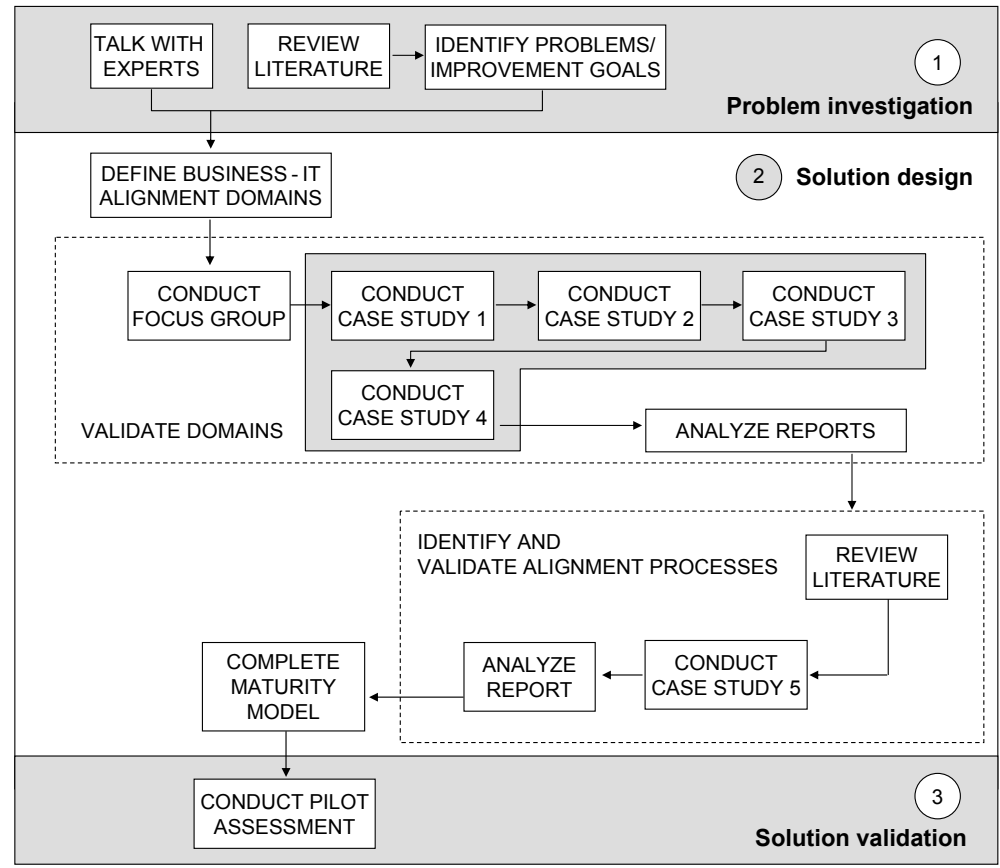

Figure 1.4: Research methodology.

We started with a literature review and interviews with experts in order to learn about current assessment approaches of B-ITa, and to identify their advantages and shortcomings (phase 1) - this phase addresses our first research question (see Figure 1.3). Based on these steps, we defined the first proposal of the B-ITa domains to include in the ICoNOs MM. Then, we confirmed the importance of those domains by conducting a focus group and a multiple case study using a realist theory-making approach [230]. The next step was to identify processes related to such domains in established CNOs. For doing this, we reviewed literature first and, then, we also conducted one case study. 


\subsection{CONTRIBUTION TO KNOWLEDGE}

Having the results of the case studies and the knowledge obtained from the review of literature as basis, we settled the domains and processes that should ultimately be included in the ICoNOs MM (phase 2) - this phase addresses our second research question (see Figure 1.3). These final domains and processes should be both complete and suitable for the purpose of assessing the maturity of B-ITa in a CNO environment. Then, we validated the complete model (phase 3 ) - this phase addresses our third research question (see Figure 1.3). Table 1.2 shows the research methods that we used for each research question. Detailed explanation of the methods and techniques that we use in our research can be found in Chapter 3.

Table 1.2: Research methods.

\begin{tabular}{|l||l|}
\hline Research question & Research method \\
\hline \hline What are the current assessment approaches of B-ITa? & $\begin{array}{l}\text { Literature review } \\
\text { (synthesis and integration } \\
\text { of information) }\end{array}$ \\
\hline $\begin{array}{l}\text { How to develop an improved approach (in our case an } \\
\text { MM)? }\end{array}$ & $\begin{array}{l}\text { Focus group, case studies } \\
\text { and literature review }\end{array}$ \\
\hline Is the improved approach valid and useful? & $\begin{array}{l}\text { Industrial trial/ } \\
\text { pilot assessment }\end{array}$ \\
\hline
\end{tabular}

\subsection{Contribution to knowledge}

Our contribution to knowledge rests on the extensive research on all aspects of B-ITa in CNOs undertaken by the theoretical and empirical studies we conducted to develop the ICoNOs MM. Specifically, the conrtibutions of this dissertation are:

- We identify a set of domains and processes that should be considered by CNOs in their efforts for achieving B-ITa.

- We provide arguments explaining why networked organizations should consider the identified B-ITa domains.

- We propose an MM that can be used by CNOs to assess processes related to B-ITa initiatives which integrate multiple perspectives.

- We present B-ITa best practices for CNOs, based on the analysis of information collected in the case studies conducted to design the ICoNOs MM.

- We provide evidence collected in six case studies that other researchers can use to form hypotheses or set up new research designs.

- We illustrate how the design of an MM involves not only literature studies but also empirical studies.

- We introduce a systematic approach in the form of a design model for the development of an MM. 


\subsection{Implications for practice}

Our research has several implications for practice. We found four domains (i.e., partnering structure, IS architecture, process architecture and coordination) that should be considered by all CNOs in their efforts for achieving cross-organizational B-ITa. The relations between these domains asserts that having a well-defined collaborative work structure as basis for the definition of the architecture of ISs and of the process architecture, helps the CNOs to react promptly to business needs. This is in a situation where coordination mechanisms are considered to manage the interaction and work among the participants in a CNO.

Since we developed an MM to assess B-ITa in CNOs, CNOs will be able to make B-ITa improvements as a whole, based on the assessment results provided by the ICoNOs MM and the B-ITa processes included in the model. In a CNO, each organization can have a different level of B-ITa maturity. This situation influences the maturity of the alignment between business and IT of the entire CNO. For example, a participant with a B-ITa maturity level 4 as a single organization can impose other participants to collaboratively achieve the same maturity level as a CNO. However, they can now base their decisions for improvements on the assessments that the ICoNOs MM can offer to them from a CNO perspective.

We consider chief officers of the participating organizations in a CNO as the key users of the ICoNOs MM assessments. This position is motivated by published results of researchers $[29,40,97,155]$, which show that the most powerful initial step to achieve B-ITa is to build strong organizational support through strong commitment of CIOs and/or CEOs. If chief officers want to improve B-ITa, they need first to assess B-ITa, and commit as B-ITa catalysts and sponsors. Applying these findings to our work, chief officers must be actively involved in the CNO B-ITa project in at least three ways: (i) influencing the CNO to use the ICoNOs MM, (ii) choosing the best team to manage the B-ITa improvement effort, and (iii) monitoring the assessment and improvement process in each B-ITa domain. As the ICoNOs MM is a continuous MM (as explained in Section 4.2.1 in Chapter 4), it lets chief officers assess each B-ITa domain separately (see Figure 1.5). This feature of the model will let CNOs focus, for instance, on those domains with a low level of maturity. Those domains that are associated with higher maturity can, then, be candidates for inclusion in later improvements efforts.

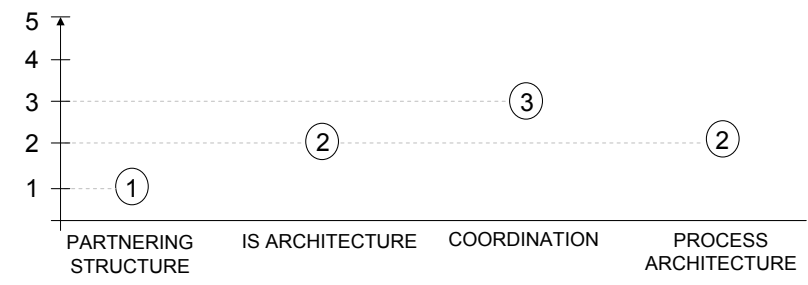

Figure 1.5: The continuous architecture of the model: An illustrative example. 


\subsection{OUTLINE OF THE DISSERTATION}

\subsection{Outline of the dissertation}

This dissertation is based on a selection of papers and reports. It is however, not organized as a collection of articles with a summary. Rather, the different articles have only served as a background and are incorporated in the text throughout the dissertation. Such an approach has the advantage that main ideas and concepts presented in the articles evolved over time and comments, for example, from conference participants where we presented them, are reflected in the dissertation. This can be a benefit considering the continuous quality assurance imposed by refereeing (and even co-authoring). The work upon which this dissertation is based is presented in Appendix A.

The remainder of this dissertation is organized in four parts. Part I presents the introductory chapters. Specifically, Chapter 2 provides the definitional foundations of this dissertation. Chapter 3 introduces the methodology we follow to develop the ICoNOs MM. Chapter 4 discusses related work addressing the first research question (see Section 1.2.2). It compares B-ITa MMs using a systematic approach for the development of an MM as basis.

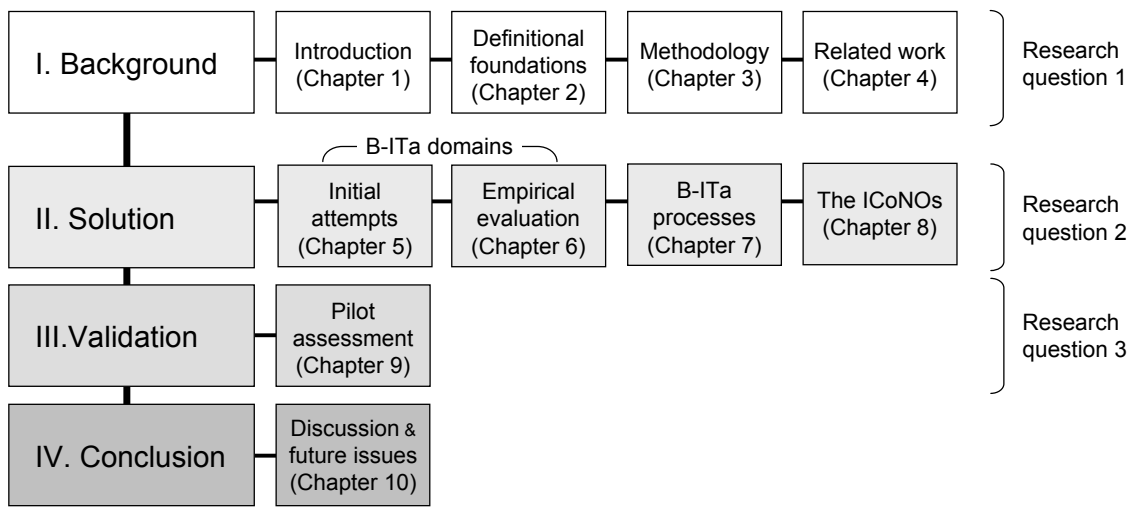

Figure 1.6: Layout of the dissertation.

Part II presents the ICoNOs MM. Chapter 5 introduces the focus group session and the first case study we conducted to identify the B-ITa domains. Chapter 6 describes the multiple case study used to validate the B-ITa domains in three CNOs. Chapter 7 (i) discusses the theories and models, developed elsewhere, that are useful to give insights for understanding the processes related to B-ITa in CNOs, and (ii) presents the case study conducted to confirm such B-ITa processes in real-life settings. Chapter 8 introduces the ICoNOs MM and elaborates on the goals to achieve in the identified B-ITa processes.

Part III of this dissertation presents the pilot assessment (Chapter 9) which have been conducted to validate the ICoNOs MM as a whole. Finally, Part IV discusses the main contributions of the dissertation and presents the inferred future work. 



\section{2 Definitional foundations}

In this chapter, we present the ideas, definitions and assumptions that are adopted in the research presented in this dissertation. The notion of B-ITa is the key concept in the research. B-ITa is often associated with an individual enterprise and not with a networked organization ${ }^{1}$. The focus in this dissertation is on B-ITa in CNOs. In reality, no organization perform succesfully all processes considered important for making B-ITa improvements. Levels of maturity can be identified. An MM can then be used to assess B-ITa and to show an improvement path recommended for a CNO that wants to achieve B-ITa. To clarify this, in the next sections we give details on, and present our definitions of, the main three concepts of the research presented in this dissertation: B-ITa (Section 2.1), CNOs (Section 2.2) and MMs (Section 2.3). A systematic literature search was undertaken to compile bibliography on these three concepts. We used the ACM Digital Library, Google Scholar, the Citeseer library, and IEEExplore for the identification of relevant work.

\subsection{Business-IT alignment}

In the famous 'IT Doesn't Matter' publication of Nicholas Carr [44], he claims that IT is commoditizing and thus available to all organizations with the result that it cannot help to differentiate organizations from each other. It is a fact that IT alone does not significantly contribute to the competitive advantage of organizations [202, 242] like in the old days when one could get an advantage in comparison to the competitors. For example, as American Airlines did with the Sabre reservation system [33], or as Dell did with its online ordering and fulfillment systems [172]. IT can become considerably complex up to the time that it strengthens problematic processes, complicates relations and interpersonal communication, and restricts the businesses. IT and business then need to be aligned in a way they help to create value in an organization. This leads to the question, what is the value that B-ITa can create?

\footnotetext{
${ }^{1}$ For instance, in the B-ITa literature survey presented by Chan and Reich [51], where they summarizes over 150 alignment articles, only the work of Lee and Leifer [176] and Scanlon [279] present in some manner a networked organization viewpoint.
} 


\section{CHAPTER 2. DEFINITIONAL FOUNDATIONS}

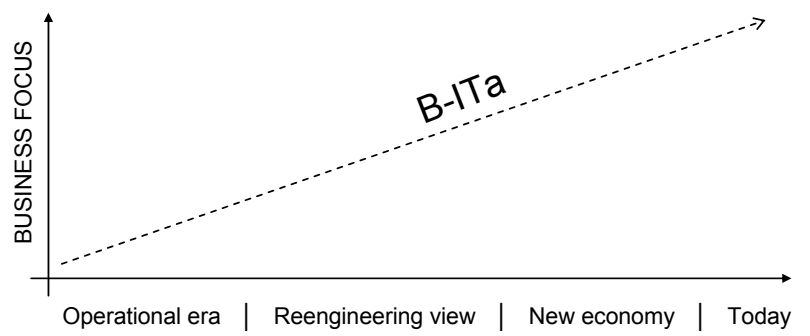

Figure 2.1: The evolution of B-ITa (adapted from [138]).

In practice, the main foci where business looks to IT to deliver value is in supporting and improving operational business efficiency and effectiveness [62], and in identifying ways to control costs, improve quality, and manage risk. However, what is B-ITa? In this section, we present a historical viewpoint of B-ITa (Section 2.1.1) and the different ways regarding B-ITa that can be found in the literature (Section 2.1.2), before introducing the definition and assumptions of B-ITa that we use in our research (Section 2.1.3).

\subsubsection{A historical viewpoint of B-ITa}

The B-ITa concept is more than 15 years old. Henderson and Sifonis [128] started to study this concept in the late 80's when "alignment emerged from a focus on strategic business planning and long-range IT planning... where IT plans were created in support of corporate strategies" [52, p. 298]. However, despite years of research, B-ITa still ranks as a major modern-day area of concern for organizations. The evolution of B-ITa closely mirrored that of IT itself: it started with the operational era and continued trough the reengineering viewpoint and the new economy (see Figure 2.1). In the operational era, IT was focused on setting up mainframes, managing networks of PCs, and backing up organizational data. IT had just a supportive role. The IT function either provided back-office support, or developed software applications to automate the back-office functions. This situation made that B-ITa alignment was seen as an event where software applications were designed to support every-day operations only.

The second phase of the evolution of IT arised from the emphasis on business process reengineering. IT was used to automate entire business processes. IT included then not only back-office functions support, but also deployment of software applications that promised to automate the organization at large. However, IT still was seen as a supportive tool that had nothing to do with the business side of organizations (even though this business side was interested in what IT could do). For example, business representatives communicated their systems requirements and the IT department took them over to develop and deploy a new software application. This B-ITa projects were not consistently successful since developing software to automate a business process did not automatically guaranteed that the process embedded in the software was an improvement over 


\subsection{BUSINESS-IT ALIGNMENT}

its manual predecessor. However, examples as American Airlines with its Sabre reservation system explicitly help to substantiate the opposite, and to visualize the transition from the operational era to the reengineering view.

In the new economy phase, IT became more important. In this phase, many organizations where involved in e-business projects. To manage such projects, they involved IT representatives in the strategic planning and gave them strategic responsibility for entire B-ITa projects. These projects try to use internet technologies to enhance (not only support or automate) business processes in order to improve the productivity or profitability of the business. Finally, with the withdrawal of the new economy burst, IT is assuming new responsibilities. Organizations are not running out on B-ITa projects. Instead, organizations are demanding real results from them. And this leads to a situation where the today's IT department has business responsibilities associated with "developing and customizing software that improves how the business functions; controlling costs ans maximizing efficiency through project management; implementing new hardware and software; supporting operations and infrastructure,... maintaining the crucial corporate data that helps managers throughout the enterprise to make intelligent and informed decisions" [138, p. 31], and managing relations with partners. Nowadays B-ITa is a way that yields competitive advantage $[155,283]$ and improves organizational performance [262, 324, 354].

The evolution of B-ITa is also visible in the several B-ITa definitions that can be found in literature. In the next section, we present some of these definitions considering the manner in which the different authors define B-ITa in terms of scope, results, and goals. The definitions serve as basis for elaborating the B-ITa definition we use in the research presented in this dissertation (see Section 2.1.3).

\subsubsection{B-ITa perspectives}

The evolution of the B-ITa concept is reflected in the number of conceptualizations of B-ITa proposed by practitioners and researchers. Table 2.1 presents a summary of several B-ITa definitions that can be found in literature. These definitions can be analyzed considering the different perspectives from which B-ITa can be seen. We explain these perspectives in turn.

\subsubsection{B-ITa as a strategic matter}

Traditionally, the B-ITa literature refers to B-ITa as 'strategic alignment'. In summary, this literature mentions that B-ITa depends on the fit between IT strategy and business strategy, and on how the IS plans are established to support such a fit [300]. Many authors in the B-ITa field approach alignment purely at the strategic level, e.g. Baets [9], Broadbent and Weill [30], Chan et al. [50], and Luftman [189]. The essence of their B-ITa conceptualizations is that business strategy must be analyzed, and then be used to define an IT strategy. However, despite the alignment between business strategy and IT strategy - mainly by means of defining IS strategy plans [16] - B-ITa continues being a problem 
CHAPTER 2. DEFINITIONAL FOUNDATIONS

Table 2.1: B-ITa definitions.

\begin{tabular}{|c|c|}
\hline Author & Definition \\
\hline Baets [9] & $\begin{array}{l}\text { The mapping between IS strategy and } \\
\text { business strategy so that IT is deployed } \\
\text { for supporting competitive advantage. }\end{array}$ \\
\hline Henderson and Venkatraman [130] & $\begin{array}{l}\text { The allocation of IT budgets such that } \\
\text { business functions are supported in an op- } \\
\text { timal way. }\end{array}$ \\
\hline Broadbent and Weill [30] & $\begin{array}{l}\text { The degree of congruence of an organiza- } \\
\text { tion's IT strategy and IT infrastructure } \\
\text { with the organization's strategic business } \\
\text { objectives and infrastructure. }\end{array}$ \\
\hline Reich and Benbasat [253] & $\begin{array}{l}\text { The degree to which the IT mission, ob- } \\
\text { jectives, and plans support and are sup- } \\
\text { ported by the business mission, objectives } \\
\text { and plans. }\end{array}$ \\
\hline Chan et al. [50] & $\begin{array}{l}\text { The situation that occurs when IS func- } \\
\text { tions are amalgamated with the most fun- } \\
\text { damental strategies and core competen- } \\
\text { cies of the organization. }\end{array}$ \\
\hline Maes et al. [192] & $\begin{array}{l}\text { A continuous process, involving manage- } \\
\text { ment and design sub processes, of con- } \\
\text { sciously and coherently interrelating all } \\
\text { components of the business/IT relation- } \\
\text { ship to contribute to the organization's } \\
\text { performance over the time. }\end{array}$ \\
\hline Duffy [95] & $\begin{array}{l}\text { The process of achieving competitive ad- } \\
\text { vantage through developing and sustain- } \\
\text { ing a symbiotic real relation between busi- } \\
\text { ness and IT. }\end{array}$ \\
\hline Luftman [189] & $\begin{array}{l}\text { A state where IT is applied in an appro- } \\
\text { priate and timely way, in harmony with } \\
\text { business strategies, goals and the needs. }\end{array}$ \\
\hline Moody [210] & $\begin{array}{l}\text { Managing the resources such as people, } \\
\text { technology, and outside resources to pro- } \\
\text { vide a set of IT services and capabilities } \\
\text { that are in line with the needs and prior- } \\
\text { ities of the businesses. }\end{array}$ \\
\hline Senn $[284]$ & $\begin{array}{l}\text { Ensuring that every single action per- } \\
\text { formed by IT individuals is focused } \\
\text { on building and delivering share- } \\
\text { holder/stakeholder value by supporting } \\
\text { business operations and/or achieving } \\
\text { business goals. }\end{array}$ \\
\hline
\end{tabular}




\subsection{BUSINESS-IT ALIGNMENT}

in many organizations. Thus, defining detailed IS strategic plans for B-ITa is important; however, is it not enough for achieving alignment [315].

\subsubsection{B-ITa as a steady state}

Besides the strategic point of view on B-ITa, B-ITa literature often implies that B-ITa is a static end state where a balance between the IT and business sides in an organization is achieved. Some of the definitions presented in Table 2.1 only present B-ITa as a desired result to be accomplished by using different models, techniques and processes [265]. For example, definitions as those of Broadbent and Weill [30], Chan et al. [50], Luftman [189] and Reich and Benbasat [253], take this viewpoint and present B-ITa as an optimal situation to achieve. Other authors, however, see alignment as a process and not as a static state (see next paragraph).

\subsubsection{B-ITa as a continuous process}

Because IT, and also business, is always in continuous evolution, B-ITa can also be seen as a process of dynamic interactions between IT and business. Research $[10,191,259]$ claims that several structures, processes and relations related with B-ITa need continuous adjustment. Therefore, B-ITa is not something reachable but it is consistently in development (as it is addressed in the definitions of Baets [9], Duffy [95], Henderson and Venkatraman [130], Maes et al. [192], Moody [210], and Senn [284]). "An issue that has remained relatively unchallenged and unquestioned is how to align ICT that is relatively fixed, once implemented in an organization, with a business strategy and associated information requirements that are constantly in need of adjustment" [113, p. 228].

\subsubsection{B-ITa as a performance indicator}

Several authors consider that B-ITa is the reflection of the business value that organizations (can) have. Their studies [155, 262, 283, 320, 324, 354] analyze the relation between B-ITa and competitive advantage or organizational performance. Tallon and Kraemer [296] added the concept of business value to the performance indicator perspective of B-ITa. They analyze the business value relating it to the IT goals that the organization must achieve, and measure the IT ROI from a B-ITa viewpoint. B-ITa can then also be seen as a measuring tool. However, how do organizations know how well their B-ITa is? The next paragraph addresses this question.

\subsubsection{B-ITa as a change driver}

In response to the question: 'how do organizations know how well their B-ITa is?', different authors [80, 95, 189, 312] have developed MMs. MMs describe the development of a specific domain over time. Based on maturity assessments, organizations know how mature their B-ITa is and they can be aware of whether a 


\section{CHAPTER 2. DEFINITIONAL FOUNDATIONS}

B-ITa process is sufficiently refined and documented so that it has the potential to achieve its desired outcomes. On the basis of the assessments of those MMs, organizations can (i) identify what alignment-related aspects/processes need improvement in order to reach a higher level of B-ITa maturity, and (ii) initiate a change process to make sure that the identified improvements occur.

\subsubsection{B-ITa as a social dimension}

Chan [48] and Huang and $\mathrm{Hu}$ [142] have found that social alignment relates to maintaining effective communication in an organization in order to establish a corporate B-ITa culture. It is important to agree on communication channels, sharing knowledge and learning $[73,138]$, and to use informal communication to adjust and control work [48, 206, 312], in order to respond effectively to business needs from an IT perspective. Reich and Benbasat [254] also studied the factors that influence the social dimension of B-ITa. They describe that B-ITa is the degree in which business and IT executives share a common vision of the ways that the IT side of an organization contributes to the success of its business side.

\subsubsection{B-ITa as a operational issue}

As it can be seen in some of the definitions presented in Table 2.1, B-ITa is not only the fit between IT strategy and business strategy. B-ITa also involves organizational issues as communication, structure and, particularly, coordination processes that operationalize the business aspect of organizations in specific actions to perform [335]. Operational B-ITa consists of aligning the operational activities of IT and business people to each other so that optimal IT support for business requirements is achieved. In this context, Peak and Guynes [231] state that B-ITa can only be achieved when IT staff can deliver quality information and quality IT products and services to the business side of organizations.

\subsubsection{Definition of B-ITa}

For the purpose of our research, we define B-ITa as

the process to make the services offered by IT support the requirements of the business - whether such services are offered individually by one participant in the CNO or collaboratively by the entire network - so that value is created for the participating organizations of the CNO.

This definition is related to the definitions given in Table 2.1 as follows: first, we do not consider alignment as a steady state but as a process of continuous improvement. This is similar to those definitions that also stress that B-ITa is a process (Baets [9], Duffy [95], Henderson and Venkatraman [130], Maes et al. [192], Moody [210], and Senn [284]); however, it differs from the rest, which sees B-ITa as a desired state. As a process, B-ITa has states that can be reached, i.e., optimal situations of B-ITa - as can be seen in some of the definitions, 


\subsection{BUSINESS-IT ALIGNMENT}

e.g., Broadbent and Weill [30], Chan et al. [50], Luftman [189] and Reich and Benbasat [253].

Second, our definition emphasizes the operational level of B-ITa. As we have discussed before, several B-ITa authors approach alignment purely at the strategic level, e.g. Baets [9], Broadbent and Weill [30], Chan et al. [50], and Luftman [189]. In contrast, the work of Maes et al. [192] seems to be applicable at both the strategic as well as the operational level, while the others seem to make no clear, explicit commitment. In our definition, with the term 'services offered by IT', we only consider ISs (i.e. software applications) as a common denominator solution to match the requirements of the business. As our work is focused on CNO settings, we explore the B-ITa concept in that context. Thereby, the 'requirements of the business' term covers the systems requirements derived from analyzing the goal $(\mathrm{s})$ and the processes of the $\mathrm{CNO}$, and the relations between the participating organizations. Finally, to be effective in a CNO, the software applications should provide value. If the value of the IT itself is not taken into account properly, the justification for the management's use of resources for B-ITa improvements is poor. By 'value' we mean the benefits obtained from the use of ISs [23, 328]. There is a great deal of literature for the estimation of IS benefits [23, 233, 329, 349]. Presenting such a literature is out of the scope of this dissertation. We only want to emphasize that being aware of the value of ISs could increase productivity in organizations $[23,158]$.

We present Figure 2.2 in order to clarify the two main terms included in our definition of B-ITa: IT services and business requirements. These terms are related to the supply-side and the demand-side in a $\mathrm{CNO}^{2}$, respectively. The supply-side provides IT services. Starting from the Frank Niessink's definition of 'service' [218]: a service is an essentially intangible set of benefits or activities that are sold by one party to another, we think of 'IT Services' as IS-enabled services supplied to a customer, that provide value. We make the explicit note that, in this dissertation, we do not make any distinction between the terms 'users' and 'customers'. For us, users, or customers, are people or organizations that use ISs in their work; therefore, they are consumers of information and they could make a large number of demands.

According to frameworks like GRAAL [316] and ArchiMate [173], IT services can be provided by the following three IT entities in an organization:

- Physical infrastructure, which consists of the computers, cables, wireless access points, printers, and user interface devices to support the running of software in an organization.

- Software infrastructure, which consists of the collection of standard generalpurpose software needed to run all the IS services. It ranges from operating systems, middleware, network software to database management software.

- Information systems, which consists of software applications that perform a set of functions on demand and that directly support the business services.

\footnotetext{
${ }^{2}$ In a CNO context, both sides can be composed by one or more participating organizations.
} 


\section{CHAPTER 2. DEFINITIONAL FOUNDATIONS}

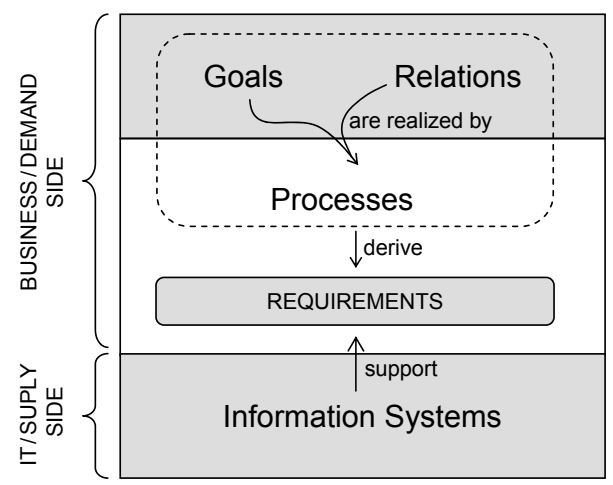

Figure 2.2: The B-ITa concept: A high level view.

For example, logistics (e.g. order handling, shipping), production systems, ERPs and CRMs.

The IT side of the B-ITa concept (see lower layer in Figure 2.2) represents the services provided by the ISs, i.e., the software applications focused on fulfill the requirements of the business. Commonly, the development of software applications focuses on achieving desired functionality instead of addressing the whole set of business requirements. To counter this situation, it is important to have a definition of the following aspects:

- the organizational goals and the relations between participants within the CNO [200] (see upper layer in Figure 2.2). The purpose is to describe what the organization wants to achieve or to avoid, and how the different organizational units, or participating organizations, are related to each other and to the goals; and

- the processes by which these goals and relations are realized (see middle layer in Figure 2.2).

The definition of goals, relations and processes forms part of the business side of the B-ITa concept. This business side can be used to drive the development of software applications since its definition is useful to derive concrete business requirements. Application development then becomes a well-organized matter of building software in accordance with the business requirements [14, 114, 258], i.e., the supply-side matches the demand-side.

Since we are interested in B-ITa in CNOs, we analyze the B-ITa concept in CNOs based on the scheme shown in Figure 1.1 (see Chapter 1). This figure shows two instantiations of Figure 2.2 (i.e., the upper two layers of ORGANIZATION A and ORGANIZATION $n$ ) in order to include the CNO perspective. Figure 1.1 is adapted from the work of van Eck et al. [316] and Derzsi and Gordijn [85] in order to 


\subsection{COLLABORATIVE NETWORKED ORGANIZATIONS}

present an overal B-ITa framework for CNOs. The horizontal layers classify entities in a service provisioning hierarchy in a business: physical entities provide services to a software infrastructure, which provides services to ISs, which provide services to businesses. In the business layer, we take four views on businesses: businesses provide services that have a utility, they perform processes to provide these services, they communicate with one another as part of performing these processes, and while doing that, they exchange data that has semantics. Participating organizations in a $\mathrm{CNO}$ need both to fit the different entities (horizontal arrows) as well as to address B-ITa (vertical arrow). Our interest is in the upper two layers of the framework (area delimited by the dotted line), because there is where business and IT alignment in CNOs takes place.

We have heretofore presented the B-ITa concept in CNOs. However, what is a CNO? In the next section, we present several definitions of cross-organizational structures in order to be able elaborating our own conceptualization of a CNO.

\subsection{Collaborative networked organizations}

Modern business markets are becoming more competitive, often due to global pressure. In response, organizations need to use innovations to create and sustain competitive advantage in order to create superior value for their customers and superior advantages for themselves [238]. Over the last few decades, we have seen that more and more organizations (i) use IT beyond purely operational and management support, and (ii) adapt their processes in order to operate smoothly and to create advantage. In particular, if we focus on IT only, with the rapid advance of technology, organizations have looked for strategic opportunities that computer networks linking organizations can provide. ISs that cross organizational boundaries have been termed as interorganizational systems (IOS) [4, 45]. Such IOS have functioned to blur the boundaries of today's organizations while they enable the flow of information from one organization to another [167]. With that goal of information flow in mind, CNOs have been created.

CNOs form the core of a new discipline $[38,39]$ that focuses on the structure, behavior, and dynamics of networks of independent organizations that collaborate to better achieve common goals. Nowadays, we can find literature analyzing several aspects of new nascent forms of organizations. In this section, we first summarize literature that tries to explain the origin of collaboration between organizations in order to better understand its nature (Section 2.2.1). Additionally, we also present definitions of new cross-organizational structures that have both common and different components (Section 2.2.2), and we analyze these definitions in order to present our own conceptualization of a CNO (Section 2.2.3).

A preliminary version of this section has been published as a Centre for Telematics and Information Technology Technical Report [267]. 


\section{CHAPTER 2. DEFINITIONAL FOUNDATIONS}

\subsubsection{The origin of CNOs}

There is a variety of reasons for the formation of CNOs. For example, networked organizations can be formed for sharing costs or for accessing complementary resources [120]. In the next paragraphs, we will study the origin of CNOs from the perspective of the most common theories used to explain cross-organizational issues: transaction costs theory (TCT) and resource dependence theory (RDT), respectively. Then, we discuss how the impact of IT on organizations also is a reason for the existence of CNOs.

\subsubsection{Transaction cost theory}

TCT is a useful theory to understand why an organization establishes its boundaries at a specific region and how it addresses the make-or-buy decision $[60,340$, $341,342,343,344]$.

The central claim of TCT is that the existence of transaction costs is the cause of the existence of organizations. In this regard, TCT can be contrasted to neoclassical economic theory. Neoclassical economic theory is principally concerned with abstract conceptualizations of markets. Neoclassical economists consider that the price mechanism acts as an instrument to coordinate the efficient allocation of resources. The term 'price mechanism' can be defined as the process by which changes in prices cause changes in resources allocation. Suppose that consumers decide they want to spend more on DVD players. So, more consumers are going to go to the stores hoping to buy DVD players. The stores see the higher demand, increase their prices, and also order more DVD players from producers. Higher profits from producing DVD players induce firms to expand production. Higher prices for DVD players are going to constrain the demand. This stimulates that the situation returns to equilibrium with more production and purchase of DVD players, and less production and purchase of other goods. Thus, 'prices' act as a 'mechanism' that guides the allocation of production resources to different economic actors.

In contrast, Coase [60] sustains that transaction costs, and not the price mechanism, determine resources allocation. He defines transaction costs as costs derived from the necessity to negotiate and to make an individual contract for each transaction. In a simple way, we can say that transaction costs are costs caused by organizing a transaction [342]. Transaction costs are the costs of searching for the right alternative, and negotiating and enforcing a contract for that.

Organizations incur transaction costs when, instead of using their own internal resources, they go out to the market for products or services. In place of buying a product on the market, the buyer (an organization) can decide to produce it in-house. This permits that the buyer saves the costs of going to the market. In this case, the buyer creates a 'company' (this means an entity, an organization) that takes care of the production of a good from the use and direction of certain resources. The buyer of a product considers the alternatives of 'buying' and 'producing' (to buy or to make), depending in each case on the costs of each 


\subsection{COLLABORATIVE NETWORKED ORGANIZATIONS}

activity: buying would lead to external costs of transaction, while producing would lead to internal costs of transaction or costs of administration within the organization. This choice is mediated by authority, by an entrepreneur, rather than by the price mechanism. An organization is considered an alternative to the market in the coordination of the resources available in the market. It is taken into account as a system of relationships that come to hand when the coordination of resources is under the direction of an entrepreneur [56].

According to TCT, a transaction should be carried out in the most economical or efficient form. The efficiency implies the reduction of both the production costs and the transaction costs [341]. If we apply transaction cost theory to a CNO, the decision to participate in such a collaboration comes from comparing transaction costs involved in joining to transaction costs involved in not joining and instead producing in-house.

Additional literature which helps understand this assertion is the literature related to markets and managerial hierarchies [201,340]. Williamson [340] proposes two groups of conditions for the existence of transaction costs:

1. conditions related to the behavior of the individual, especially those associated with the bounded rationality of the human being, the opportunism of the involved parts and dignity; and

2. conditions related to the environment of the transaction, in particular, uncertainty about the future and the existence of small groups of organizations with which to work, and asset specificity and frequency of the transactions.

Focusing on these last conditions, TCT establishes that the make-or-buy decision will be influenced by three attributes of the transactions themselves: asset specificity, frequency, and uncertainty. If the transactions rate high in these attributes, then it is more probable that the organization will internalize such transactions, i.e., it will produce in-house. Each of these three attributes concerns an important dimension of the activities within the organization [8]. To illustrate this, consider a customer-supplier relationship. First, high asset specificity means that an asset is transaction specific and cannot be redeployed, e.g., when the supplier has produced an asset highly customized to the needs of only one customer, that it is not possible to use it in another context. Second, high frequency means that the supplier and the customer conduct many transactions for a specific required component, raw material, or service. Finally, high uncertainty means that the customer is obtaining the service or product from a supplier in a risk-characterized market environment. If, on the other hand, the transactions rank low on these three attributes, the organization will tend to obtain the services or products from other organizations in the market. This situation leads to the creation of CNOs.

Williamson also studies the term "hybrids". He defines hybrids as: "various forms of long-term contracting, reciprocal trading, regulation, franchising, and the like" [343, p. 280]. In this alternative form of economic organization, the buying organization and the selling organization establish, for the provision of a 


\section{CHAPTER 2. DEFINITIONAL FOUNDATIONS}

product, a contract with the conditions of the transaction (price, quality, date of delivery). A CNO can be considered as an hybrid where two or more organizations can be involved.

At this point, we can say that transaction cost theory can be seen as a base theory to think that the most economical and efficient form to carry out a transaction is by means of collaboration between organizations. However, organizations have no goals that are purely economic. Therefore, TCT is not sufficient to explain the origin of CNOs.

\subsubsection{Resource dependence theory}

An additional theory that helps to explain the existence of CNOs is RDT. In contrast to TCT, RDT assumes exchange is affected by social factors and focuses on the social context of the organization's relationships with their environment. RDT suggests that organizations enter in tie-ups with other organizations in response to the challenges posed by the dependences that shape their common environment [234]. According to RDT, complementarity between organizations arise when (i) there is a gap between the specific resources managed by each organization and those they need to perform their activities and (ii) this gap can be filled, at least partially, by accessing resources managed by other organizations while offering something of value in return [121]. In such a condition, organizations begin to collaborate.

RDT centers on how some organizations become reliant on others for needed inputs such as goods and/or services, and on how they can manage such relations. RDT acknowledges that a single organization cannot produce or own all the required resources for its operations. The organization is forced to acquire these resources from other organizations in its environment. RDT emphasizes the fact that no organization is self-sufficient; no organization is able to generate all necessary resources by itself. That is the reason why organizations need to be connected with other organizations to make trade-offs while they assure their survival. RDT analyzes two aspects: the factors that decide the degree of dependence of an organization with respect to others, and the actions taken to address that dependence [208]. The degree of dependence of an organization on others is determined by the significance or otherwise of the resources the organization needs to perform activities in order to create and sell its own products or services. So, the organization needs to identify those processes it could best perform together with other organizations and to reinvent itself by establishing cross-organizational processes [47].

RDT has its origin in the power-dependence relation theory of Emerson [100], and it is suited within the approaches that study the relation between the environment and organizations' performance. In summary, this approach holds that organizations must study themselves in relation to the organizations with which they want to share resources. In such a study, organizations need to give special attention to external control which they could face when their processes depend partially, or completely, on other organizations' resources. Without any elabo- 


\subsection{COLLABORATIVE NETWORKED ORGANIZATIONS}

ration, RDT holds that organizations form CNOs not mindfully only, but with hesitation. This results from the perception that commonly organizations have regarding CNOs, i.e., organizations see CNOs as groups of liabilities and external

control. Therefore, participating in a CNO can result in a loss of power and control in the own decision-making process. In RDT power is based on resource exchange between parties. That is, the more ORGANIZATION A is dependent on a resource of ORGANIZATION B, the higher the power that ORGANIZATION B has over ORGANIZATION A $[65,100,170,234]$. Yet, we believe that organizations form CNOs not mindfully but optimistically. Organizations realize that to be more competitive they must have access to valuable resources and that CNOs are commonly the best way to access such resources.

\subsubsection{Information technology impact}

After analyzing TCT and RDT, we want to present in the next paragraphs the relationship between IT and the development of organizational forms [187]. We in particular present how IT also is able to promote new forms of collaboration between organizations.

Malhotra discusses in his work [196] that one of the uses of IT within organizations is to achieve external business communication goals. External business communication, as the term suggests, includes all the transmission of information and meaning that occurs in a business context, i.e., from one business to another business. Over the last few decades, we have seen that more and more organizations use IT beyond purely internal operational and management support, but also for business communication. In particular, with the rapid advance of the technology, organizations have looked for strategic opportunities that computer networks linking organizations can provide. These increasing interdependencies demand more flexible and adaptive organizations [197]. From the IT impact perspective, this is the origin of the CNO concept. In fact, IT allows that organizations design and plan the dependencies between them when collaborating with each other to achieve joint, strategic goals in order to gain competitive advantage [338, 339].

The reduction in costs is the initial motivation to think on cross-organizational structures where IT facilitates the coordination between the organizations along the CNO [261]. Nevertheless, IT also is itself a motivation to think on the origin of the CNO concept. We know that IT is a vital part of most organizations. We cannot imagine the existence of any significant company without IT. This relation between IT and companies' existence conducts to think that if IT evolves, the company also needs to evolve by adjusting its structure to the new technologies, e.g. network environments where the sharing of information between organizations is a crucial factor to be competitive in the real world. In this regard, Tapscott [297] and Fulk and DeSanctis [110] remark that electronic communication, and computing power in general, stimulates change in business models. By business model, we mean in this point the method of doing business: how the organization transacts with the environment to create value [159,252]. The business 


\section{CHAPTER 2. DEFINITIONAL FOUNDATIONS}

model of an organization then determines its organizational structure [159, 217]. Consequently, we can find the relation:

$$
\text { IT } \rightarrow \text { business model } \rightarrow \text { organizational structure } \mapsto C N O .
$$

Computer networks provided the basic concepts, and the necessary infrastructure, to begin to work in more horizontal organizational structures, namely CNOs. Furthermore, these days when the operating feasibility of CNOs has been proved, IT accentuate the trend towards them [261].

After analyzing the origin of CNOs from three different perspectives (i.e., TCT, RDT and IT impact), in the next paragraphs we present definitions of concepts related to CNOs to clarify ideas around the CNO term and to arrive at our own CNO conceptualization.

\subsubsection{CNO-related terms}

In this section, we present definitions of several terms that are commonly used to describe cross-organizational structures. Sometimes, these terms are cited as similar to the term CNO. For that reason, we tried to find their principal components attempting to see if they are indeed similar, and to determine interrelationships among them (see Section 2.2.3).

\section{Networked organization}

We begin with the definition of a network. According to Lipnack and Stamps [182, p. 161], "a network is a web of free-standing participants cohering through shared interests and values"; networks are decentralized organizations.

Having this definition in mind, the first compound concept we define is that of the networked organization. A networked organization is a situation that come up when independent people and groups, linked across boundaries, work together for achieving a common purpose [183]. Lipnack and Stamps also explain that a networked organization has multiple leaders, lots of voluntary links between participants, and interacting levels. By interacting levels, they mean levels of successive inclusion in which, like everything complex in nature, networks are organized. "In the context of systems, which networks are, levels mean sets within sets like cells in tissues in organs in organisms" [183, p. 52].

This term - networked organization - is more related to the concept of work teams within organizations [183] because it is about how people can effectively work together within a variety of group contexts in an organization. However, this term can extend its borders beyond single organizations because the future of organizations depends on the collaborative work among directors, employees, suppliers, distributors, clients, government and competitors.

\section{Networked enterprise}

Steen et al. [293] state that supply chains, electronic markets and virtual enterprises are all examples of networked enterprises. The definition of this concept 


\subsection{COLLABORATIVE NETWORKED ORGANIZATIONS}

was conceived to be intentionally broad so as to cover all possible forms of crossorganizational cooperation:

"A networked enterprise is any undertaking that involves two or more interacting parties" [293, p. 1].

Steen et al. use the term 'interacting parties' to refer to business units or organizations. This definition mentions the term 'undertaking' which consequently involves a purpose. This point is important for the next definition.

\section{Virtual enterprise}

As we just mentioned, Steen et al. [293] state that virtual enterprises are an example of networked enterprises. This term - virtual enterprise - needs to be defined as a separate term because people commonly refer to virtual enterprises when they want to talk about cross-organizational structures. "A virtual enterprise is a temporary network of autonomous firms dynamically connecting themselves stimulated and driven by a business opportunity arising on market. Every member makes available some proprietary subprocesses and part of its own knowledge. When the business opportunity is over, members disconnect and look for new businesses" [12, p. 2].

A virtual enterprise is commonly seen as reconfigurable and its boundaries are more blurred than other cross-organizational structures. According to Barbini and D'Atri [12], a virtual enterprise is an informal cooperation between organizations without formalization of tasks and structure. We do not agree with this statement because we think formalization of tasks and most importantly structure are necessary for working in any kind of cooperation between organizations (see Chapters 5 and 6).

\section{Extended enterprise}

Barbini and D'Atri [12] also state that an extended enterprise is another new cross-organizational configuration. They describe an extended enterprise as a network of firms formally structured around a focal organization which deploys technology in order to manage the network to achieve a larger and more flexible supply chain. "The development of an extended enterprise requires relevant investments on infrastuctures and on coordination agreements, hence it is usually intended to operate for long period of time" $[12$, p. 6].

Besides the point of view of Barbini and D'Atri, the term extended enterprise is usually used to represent the concept that an organization is composed not just of its employees and executives, but also of its business partners, its suppliers, and its customers; where each party has equal participation.

\section{Value constellation}

Having the definition of extended enterprise in mind, it also is valuable to define the term value constellation. Normann and Ramírez [221] introduced this 


\section{CHAPTER 2. DEFINITIONAL FOUNDATIONS}

term to define groups of enterprises that together satisfy a consumer need, where each enterprise uses its own expertise, products, and services. They state that in the actual competitive environment, successful organizations are focus on the value-creating system, within which different actors - suppliers, business partners, customers - work together to co-produce value. "To put it in another way, successful companies conceive of strategy as systematic social innovation: the continuous design en redesign of complex business systems" [221, p. 65] to create valuable objects.

Value constellations are a successor of the value chain. The value chain concept points that value is added, in sequence, by suppliers along the chain. According to Normann and Ramírez, that creation of value is not linear anymore; however, it is done within constellations of organizations. Such constellations focus on the products and services that organizations exchange, and on more long-term-relations [118].

\section{Joint venture}

A joint venture is a traditional form of partnership, a minimal network, where two or more organizations join resources to work together [183]. In a joint venture, organizations agree on sharing capital, technology, human resources, risks and rewards in a formation of a new entity under shared control to pursue a mutual strategic goal.

In the Dictionary of Business and Management [346], we can also find that a joint venture is "nearly always agreed for a fixed time-frame - commonly three to five years in the west, but often ten, fifteen or even twenty years in Asia -" with options for either terminating the venture or renewing it for a further period if participants agree on that.

\section{Business webs}

Tapscott et al. [298] define business webs as fluid congregations or collaborations of businesses that come together loosely or in highly structured networks to accomplish shared agendas. Based on their research and on a large number of case studies, they argue that business webs are the new model for creating wealth in the new digital economy where businesses use the internet for their primary business communication and transaction goals. So, business webs are partner networks linked digitally to generate value for the customers and shared wealth.

\section{Symbiotic partnership}

When an organization builds strong relations by integrating legally and economically other independent organizations as part of the accomplishment of its own tasks, such an organization is establishing a symbiotic partnership. This kind of partnerships "create reciprocal dependencies providing mutual advantage. In order to avoid or at least to limit the opportunistic exploitation of these depen- 


\subsection{COLLABORATIVE NETWORKED ORGANIZATIONS}

dencies by one partner, symbiotic arrangements are usually planned as long-term relationships" [339, p. 209].

The symbiotic partnership is characterized by reciprocity (one party helps the other in return for their help) and blurriness of boundaries and spheres of responsibility.

\subsubsection{Definition of CNO}

After presenting the previous definitios, we analyze the principal elements that we can find in each of them. With this analysis, we determine interrelationships among the definitions to provide a broad and precise, new definition of the term CNO. To achieve a significant summary of the cross-organizational structures mentioned, we have established three 'indicators', namely the principal components found in the definitions: interaction of parts, common purpose and duration of coorperation. Table 2.2 presents our findings. The indicator 'interaction of parts' does not need to be explained because it is a component that can easily be found in all the presented definitions, which is not the case for the other two indicators. Table 2.3 shows the terms previously described together with the principal components found in their definitions.

Table 2.2: Matrix concept-indicator: The summary of findings.

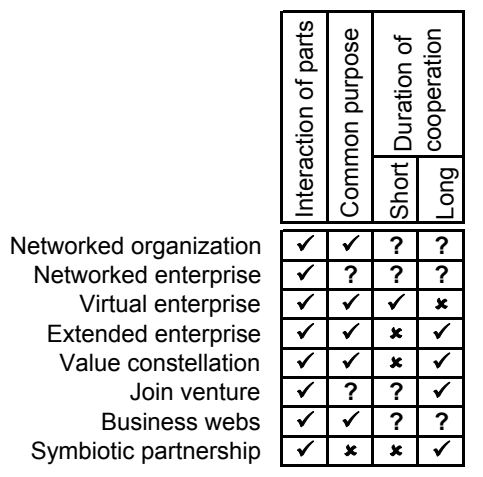

Legend: $\checkmark$ Term mentioned, or found, in the definition

* Term does not apply

? Term is not mentioned, a deep study is required

We begin with the indicator 'common purpose'. The definition of a networked organization literally mentions the term 'common purpose'. As Lipnack and Stamps claim, a common "purpose is the vital spirit of a network expressed as a unifying aim" [183, p. 41]. Most cross-organizational structures require some well-defined motivation for formation. Participating organizations have their individual purposes. However, if they want to collaborate, they need to formulate 


\section{CHAPTER 2. DEFINITIONAL FOUNDATIONS}

Table 2.3: Cross-organizational structures and their principal components.

\begin{tabular}{l|l|l|} 
Concept & Principal component \\
\hline networked organization & $\begin{array}{l}\text { independent nodes linked } \\
\text { across boundaries } \\
\text { work for a common purpose }\end{array}$ \\
\hline networked enterprise & $\begin{array}{l}\text { interacting parties } \\
\text { virtual enterprises }\end{array}$ & $\begin{array}{l}\text { - dynamically connected } \\
\text { autonomous firms } \\
\text { business opportunity } \\
\text { temporary network }\end{array}$ \\
\hline extended enterprise & $\begin{array}{l}\text { network of firms } \\
\text { long period of time relation } \\
\text { achieving a more flexible } \\
\text { supply chain }\end{array}$ \\
\hline value constellation & $\begin{array}{l}\text { groups of enterprises } \\
\text { satisfying a consumer need } \\
\text { and co-producing value } \\
\text { long-term-relations }\end{array}$ \\
\hline joint venture & $\begin{array}{l}\text { two or more companies } \\
\text { form a separate entity } \\
\text { fixed time-frame relation }\end{array}$ \\
\hline susiness webs & $\begin{array}{l}\text { collaboration of businesses } \\
\text { generating a shared wealth }\end{array}$ \\
\hline $\begin{array}{l}\text { partnership between organ } \\
\text { izations } \\
\text { long-term relationships }\end{array}$
\end{tabular}

a clear-enough unifying purpose toward which they strive. This formulation is not an easy task since organizations are cognitive entities interacting socially. So, the problem of social interaction between cognitive entities is, according to Castelfranchi [46], how to obtain that another organization does or does not something? How to induce the other to believe and even to want to achieve the same of our organization? The answer can be: communication. However, communication can only inform the organizations' purpose. Communication does not assure the formulation of a unifying purpose. In order to formulate such a purpose, organizations need power over the other participants in order to influence them. Castelfranchi states that the most important basis of the power of an organization is the fact that probably also the actions of such an organization are potentially interfering with the purpose of the other participating organizations. Participants depend on other participants for their purpose. So, organizations can induce others to establish a social goal, a common purpose, which is the overall purpose of the participating organizations as a group.

The term 'business opportunity' found in the definition of virtual enterprise can be translated into a purpose. The same can happen with the extended enterprise, value constellation and business webs definitions because 'to achieve more flexible supply chain', 'to satisfy a consumer need and co-produce value' and 'to generate a shared wealth' are their specific common purpose, respectively. We make the note that the common purpose in a CNO is not necessary the purpose of 


\subsection{COLLABORATIVE NETWORKED ORGANIZATIONS}

all the participating organizations. The common purpose is an agreement among the customer-faced organization and its direct partners. This common purpose might include also other participants in the CNO, but not necessarily.

The indicator 'duration of cooperation' can only be found in five definitions, namely the definitions of virtual enterprise, extended enterprise, value constellation, joint venture and symbiotic partnership. A deeper literature study is required to determine if this indicator could be related to the other definitions. There is another term that does not appear in Table 2.2 but does need to be mentioned. It is the term 'exchange of resources' found in the definition of the joint venture. This term is important because for any collaboration between organizations to achieve a common goal, beside the formalizations of tasks and structure, the exchange of capital, technology, human resources, risks and/or rewards is necessary. So far, we have already identified a point that we always need to have in mind in order to obtain our own idea of a CNO: the essential parts of the definition (i.e., common purpose, interaction of parts, duration of coorperation).

It must be noted, that none of the presented definitions has taken directly into account the IT viewpoint. As we have already asserted, IOS have functioned to blur the boundaries of today's organizations. This is a starting point to think about the term CNO. In our context, networks exist when different organizations decide to collaborate by means of IT [336]. A CNO uses IT to integrate cross-organizational functions enabling the automation of coordination [64]. Considering the definitions that we have presented, and in order to have a complete definition of a $\mathrm{CNO}$, we define a CNO to be any:

"mix-and-match" network of profit-and-loss responsible organizational units, or of independent organizations, connected by IT, that work together to jointly accomplish tasks, reach common goals and serve customers over a period of time [267].

With the term 'period of time', we want to include the dynamic behavior of networks in this definition. We know networks are dynamic and can change from moment to moment. So, as we can find it in the definition of a virtual enterprise, organizations work together during the time that an interesting business opportunity exists. When the business opportunity is over, the CNO dissolves while, perhaps, the participating organizations are active in other CNOs or look for new complementarities that allow them to participate in new 'business' opportunities. Our interest is in IT-enabled CNOs, i.e., collaborations that are made possible by IT where the participants interoperate with each other by means of ISs. We believe that IT makes global competition and collaboration possible, forcing organizations to focus on what they can do well and facilitating collaboration between organizations with complementary competencies.

So far, we have discussed the concepts of B-ITa and CNOs. In the next section, we present (i) a definition of an MM and (ii) a summary of the most well-known maturity model, i.e., the Capability Maturity Model Integration (CMMI). The 


\section{CHAPTER 2. DEFINITIONAL FOUNDATIONS}

rationality for the development of an MM in the context of B-ITa in CNOs is presented in Chapter 4 when we discuss the current MMs of B-ITa processes.

\subsection{Maturity models}

Although the extended and effective use of IT in organizations, predictable results commonly depend on individual experts. However, the use of experts does not automatically assure success and continuous improvement throughout an organization. As a result organizations are more and more using MMs for assessing and improving processes on the premise that the quality of a service or product is highly determined by the quality of the processes performed to develop and maintain such a service or product in the organization.

MMs describe the evolution of a specific entity over time. Usually, this entity is an organizational area or function. MMs have been developed to assess specific areas against a norm. Based on maturity assessments, organizations know the extent to which activities in such areas are predictable. That is, organizations can be aware of whether a specific area is sufficiently refined and documented so that the activities in this area now have the potential to achieve its desired outcomes. MMs are then descriptive and normative, but not prescriptive. They are descriptive models in the sense that they describe essential characteristics or processes that are expected to distinguish an organization at a specific maturity level. Additionally, MMs also are normative because they commonly present practices that characterize the 'normal' behavior that is expected in an organization in particular areas; they provide a minimum set of attributes that need to be adopted for improvements. However, MMs are not prescriptive. They do not tell an organization how to improve. MMs focus on "what" not on "how". They describe an organization at each maturity level (presenting practices that should be adopted) without prescribing the specific means for getting there.

MMs present the evolution of an area over time until it reaches its highest maturity level. This does not mean that such an area is always going to reach the highest level of maturity. An MM shows the way to reach the most mature status. Organizations decide what maturity level is the level that better meets their goals. Essentially, MMs make it easier for organizations to establish goals for process improvement and identify opportunities for optimization, since these models describe basic attributes that are expected to characterize a particular area for each maturity level. By comparing the characteristics and attributes of an organization with an MM, the organization can identify how mature it is in order to increase its process capability: first, establishing goals for the improvement of processes and then, taking action to achieve them.

A maturity model also:

- allows an organization to benchmark itself with other internal or external organizations for the purpose of improving its own processes.

- offers an improvement path to organizations so that they can set priorities 


\subsection{MATURITY MODELS}

for process improvement actions. These improvement actions will permit an organization to achieve a higher level of maturity increasing its possibility of predicting. If an organization's maturity increases, then the definition of results becomes more accurate [115].

- allows a possible interested party to have certainty on the potential of the organization. Depending on the level of maturity that an organization has, an external party (another organization, a stakeholder, a client, or a supplier) can have a clear idea about the organization's capabilities, in order to decide to have economic dealings with such an organization.

The first well-known maturity model was the $\mathrm{SW} \mathrm{CMM}^{3}$ proposed by Carnegie Mellon University's SEI. This model identified, specifically for software production, several levels of software process management sophistication. When maintaining the model, the SEI observed that organizations wanted to focus their improvement efforts not only in software production but also across different organizational functions. In response, the SW CMM has been superseded by the CMMI. Because some design issues of the ICoNOs MM (e.g. the structure, the levels, the architecture) are based on the CMMI (see Section 4.2 in Chapter 4), we briefly present this model in the next section.

\subsubsection{The CMMI}

The CMMI is the most widely recognized global standard and implemented software process improvement framework. CMMI is the most recent model developed by Carnegie Mellon University's SEI (http://www.sei.cmu.edu), and embodies the world's state-of-the-art practices that provide guidance for managing the development process, for delivering services with organizations and to external parties, and for enabling decisive acquisition leadership [58]. The CMMI is built on the basis of the knowledge and experience of many experts in the disciplines that the model involves. The CMMI describes how organizations can improve their processes and, at the same time, it also offers the possibility of expressing the maturity of the processes in a measurable value.

There are three areas of interest covered by CMMI: development, acquisition and services. The current version of CMMI therefore covers three models:

- CMMI for Development (CMMI-DEV). This model addresses product and service development processes.

- CMMI for Acquisition (CMMI-ACQ). This model addresses the acquisition management of products/services.

- CMMI for Services (CMMI-SVC). This model addresses internal and external service delivering.

\footnotetext{
${ }^{3}$ More information on http://www.sei.cmu.edu/cmm/
} 


\section{CHAPTER 2. DEFINITIONAL FOUNDATIONS}

\begin{tabular}{|c|c|}
\hline Optimizing & Level 5 \\
\hline Quantitatively managed & Level 4 \\
\hline Defined & Level 3 \\
\hline Managed & Level 2 \\
\hline Initial & Level 1 \\
\hline
\end{tabular}

Figure 2.3: The levels in the CMMI.

Regardless of which model an organization chooses, the CMMI guidelines should be adapted to each individual organization according to its goals.

The CMMI uses five levels of maturity (see Figure 2.3). At the lowest level, the processes are unpredictable, there is no structure and they are poorly controlled and reactive. The success of a project in organizations at level 1 depends on the skills of a few key players. The organization is purely people-dependent ${ }^{4}$. Level 2 is the Managed level. At this level the organization starts looking at its processes as the main cause behind its inefficiencies instead of thinking that people are the problem prime mover. Therefore, the organization starts to define the processes and to set up the basic controls for them. At level 3 the processes become completely defined and maintained on a organization-wide basis. Moreover, projects tailor their processes from organization-wide standards. In general, the processes are more adaptive. At level 4 or Quantitatively Managed level, the organization focuses on managing the processes using statistical tools. Finally, an organization at level 5 directs its efforts to continuously improve the processes. The organization depends on innovation and continual improvements.

The processes in the CMMI can be organized into two architectures (or 'representations' in CMMI-words): 'staged' and 'continuous'. Both architectures provide essentially the same content and use the same model components. However, they are organized in different ways. In the staged architecture, process areas are organized by maturity levels. Each level is a layer for process improvement using a predefined improvement sequence. It describes an organization at each maturity level and shows an exact order to perform processes. This architecture focused on the levels of the MM. For instance, before reaching level 3, an organization needs to achieve successfully what is mentioned in level 2 for all the domains included in the model.

In the continuous architecture, the levels are used to describe a sequential order for approaching improvement within each process area. It allows selection of the order of improvement that best meets the objectives of organizations. This representation is used to appraise the process areas in the domains and gives more flexibility to the organizations to choose areas to focus on. It does not have a strict order to follow when performing processes.

\footnotetext{
${ }^{4}$ In reality, all organizations are people-dependent. However, from the CMMI perspective this means the risk to fail is very high.
} 


\subsection{Summary}

In this chapter, we provided an overview of the definitions and assumptions of the three main concepts of our research: B-ITa, CNOs and MMs. This serves as definitional background for the rest of the dissertation. We started presenting the different perspectives from which B-ITa can be seen and several B-ITa definitions. That helped us to create our own conceptualization of B-ITa. For us, B-ITa is a process to make IS-enabled services (i.e., software applications) support the requirements of the business. It does not matter whether such services are individually or collaboratively offered in the CNO. What is important is that this alignment between IT services and business requirements creates value for the participants in the CNO.

Furthermore, in order to present what a CNO for us is, we first presented the roots of the CNO concept using two theories: transaction costs theory (TCT) and resource dependence theory (RDT). We also discussed how the IT impact on organizations is, in its own, a reason for the existence of CNOs. A survey of crossorganizational structures definitions helped us to identify several elements that had to be included in our CNO definition. For us, a CNO is any "mix-and-match" network of profit-and-loss responsible organizational units, or of independent organizations, connected by IT, that work together to jointly accomplish tasks, reach common goals and serve customers over a period of time.

Finally, we present the nature of MMs and, specifically, we briefly introduced the CMMI because some design choices of our ICoNOs MM are based on this well-known model. 



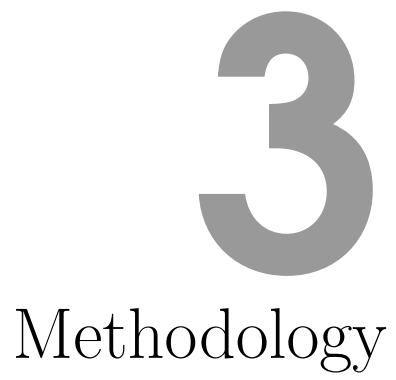

In this chapter, we describe the methods and techniques that we used in our research for the development of the ICoNOs MM, providing justification for their utilization. Furthermore, we also discuss certain issues that could have an impact on the final results of our research, for example because they impact the extent to which our results are generalizable. We do not pretend, however, to explain in detail what we exactly did when using these methods and techniques. Such explanations can be found in the following chapters of this dissertation where we present in detail the steps we followed to develop our model.

As presented in Chapter 1, the problem that we addressed in our research is that in a CNO context, it is complicated to have improvements in the way of using ISs properly so that they support business requirements (i.e., it is complicated to have improvements in B-ITa). These requirements are derived from analyzing the goal $(\mathrm{s})$ and the processes of the $\mathrm{CNO}$, and the relations between the participating organizations. We then dealt with the development of the ICoNOs MM in order to assess the maturity of B-ITa in CNOs providing a list of processes that can be used for B-ITa improvements. We started the development of our model being interested in the built-in design knowledge of the current assessment approaches of B-ITa. We were particular interested in identifying and reusing this knowledge in the design of the ICoNOs MM. In this way, we avoided developing a new approach from scratch which, in turn, made the development process cheaper and faster. Therefore, the first research question that guided the starting phase of the study presented in this dissertation was (see Figure 1.3):

\section{Q1 What are the current assessment approaches of B-ITa?}

The purpose of this question was to identify advantages and disadvantages of current B-ITa assessment approaches in order to define the improvement goals and reusable knowledge. We then started to investigate:

\section{Q2 How to develop an improved approach (in our case an MM)?}

We needed to find a systematic approach to transform the design knowledge into an MM. Finally, after developing a new MM to assess B-ITa in CNOs, we evaluated the model addressing the next question: 


\section{CHAPTER 3. METHODOLOGY}

\section{Q3 Is the improved approach valid and useful?}

These research questions drove the development of the ICoNOs MM. They were answered by using an appropriated methodology, which included empirical data collection and analysis techniques, as presented in the next sections in this chapter. First, in Section 3.1, we elaborate on the type of the research we conducted. Then, in Section 3.2 we present the methods and techniques which composed our research approach. For each method or technique, we provide a description and our motivation for using it. Finally, in Section 3.3, we introduce construct, internal and external validity, and we describe how we addressed each of them.

\subsection{Type of research}

The research presented in this dissertation can be categorized as conceptual [211, 251], qualitative [89, 305] and interdisciplinary [186, 263]. It is conceptual research because in two steps of our research (namely, the literature reviews in the problem investigation and the solution design phases - see Figure 3.1) we studied and analyzed concepts and literature related to B-ITa MMs and to the domains that could compose B-ITa, respectively. The objective was to show how important concepts developed elsewhere may help to identify improvement goals and reusable knowledge to address in the design of the ICoNOs MM. In this research, we synthesized and integrated these concepts and literature in order to develop our MM.

It can be argued that conceptual research is a non-empirical research method that only is based on subjective reviews (rather than on systematic procedures) and, therefore, it is not widely used in the IS discipline [211, 295]. However, we believed that conceptual research helped us to give a theoretical basis to our research, as it happens in other disciplines ${ }^{1}$. According to Mora et al. [211], conceptual research can be classified in behavioral and design research. Conceptual behavioral research is related to explorative and descriptive studies of conceptual entities; conceptual design research accounts for the design of conceptual artifacts (constructs, frameworks, theories, models, methods) without involving empirical evaluations. In our research, we have covered both types. We conducted conceptual behavioral research in order to describe the current MMs that can be used to assess B-ITa and the MMs that have been developed to assess specific CNO domains (see Chapter 4). We present what is known and, after comparing several models, we also show what the improvement goals (research challenges) are to address in the development of the ICoNOs MM. Furthermore, conceptual design research was conducted to analyze different theories and models related to B-ITa domains in order to identify process areas to include in our model (see the literature review conducted in Chapter 7).

Our research also falls into the category of qualitative research. Our choice for qualitative research was motivated by the following two issues:

\footnotetext{
${ }^{1}$ Several examples can be found in Mora et al. [211].
} 


\subsection{RESEARCH APPROACH}

- The research problem investigated.

Because we developed a new MM to address the fact that there was no MM to assess B-ITa in CNOs, our choice for qualitative research was influenced by our desire to examine what a CNO context meant to real-life organizations and how these organizations dealt with this CNO context in the way they worked in a specific B-ITa project. We wanted to study their work practices and processes. We determined that the best way to obtain the information we sought was by interviews with people involved in such projects (both formally and informally), observation, and analysis of documents - as presented in Section 3.2.2. The use of these data-gathering techniques results in mostly qualitative data $[107,119,226]$.

- The uncertainty of the phenomena.

As we wanted to investigate B-ITa in real-life (constantly changing) CNOs, it was expected that we had to analyze in depth complex phenomena dealing with significant qualitative data. This, together with the fact that our research investigates a topic that is brand-new in nature, supported our decision to use case studies as primary research method in the development of the ICoNOs MM (see Section 3.2.1). Benbasat et al. [15] claims that the case study approach is appropriate for IT research areas in which few previous studies have been carried out.

Finally, we want to emphasize the interdisciplinary nature of our research. Interdisciplinary research is the most commonly used research type in design research [7]. It involves the use of ideas of several disciplines to enhance solution design within another discipline. For our purposes, a discipline is a branch of knowledge composed of ideas, problems, theories, and/or methods of investigation. Our research is interdisciplinary because it brings together in a novel way distinctive components of different disciplines (e.g. B-ITa, CNOs, MMs, ISs, coordination). Working with different paradigms complicated defining what kind of information was relevant and what kind of analysis was the best for our research. However, interdisciplinary research itself helped us to cover different aspects that could compose B-ITa in CNOs which, in turn, supported the development of a state-of-art literature-based MM.

\subsection{Research approach}

The approach we adopted for this research is presented in Figure 3.1 (which is repeated from Chapter 1 for ease of reference). As already indicated in Chapter 1, our research comprised three main phases:

1. problem investigation

This phase helped us to answer our first research question. We started with a conceptual research (literature review of related work - see Chapter 4) 


\section{CHAPTER 3. METHODOLOGY}

and interviews with experts in order to learn about current assessment approaches of B-ITa, and to identify their advantages and disadvantages, and knowledge that we could use in the development of our MM.

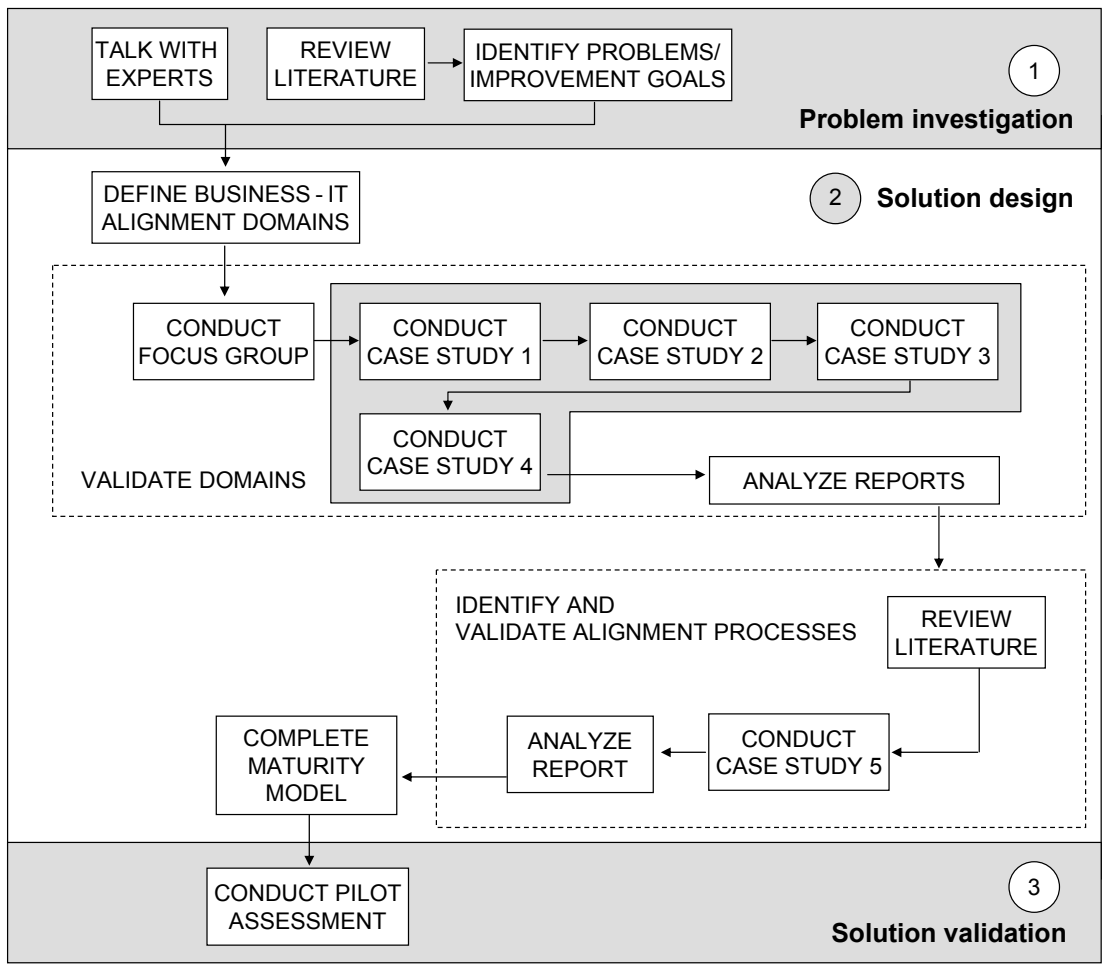

Figure 3.1: Research methodology (taken from Chapter 1).

2. solution design

This phase addressed our second research question. Based on the previous phase, we defined the first proposal of the B-ITa domains to include in the ICoNOs MM. Then, we confirmed the importance of those domains by conducting a focus group and a multiple case study using a realist theorymaking approach [230] as presented in Chapters 5 and 6. The next step was to identify processes related to such domains in established CNOs. For doing this, we reviewed literature first and, then we also conducted one case study (see Chapter 7). Having the results of the case studies and the knowledge obtained from the review of literature as basis, we settled the domains and processes that should ultimately be included in the ICoNOs MM. We make the explicit note that although the case studies in this phase were conducted having validation as purpose, they validated components of our model and not the MM itself (which is addressed in the next phase). 


\subsection{RESEARCH APPROACH}

3. solution validation

Finally, we validated the complete model by conducting a pilot assessment in a real-life CNO.

In each of these three phases we used different research methods according to the essential characteristics of each phase. The next section presents these methods.

\subsubsection{Research methods}

In order to show the research methods that we have used in each research question and in turn to make this section self-contained, we present Table 3.1 once more.

Table 3.1: Research methods (taken from Chapter 1).

\begin{tabular}{|l||l|}
\hline Research question & Research method \\
\hline \hline What are the current assessment approaches of B-ITa? & $\begin{array}{l}\text { Literature review } \\
\text { (synthesis and integration } \\
\text { of information) }\end{array}$ \\
\hline $\begin{array}{l}\text { How to develop an improved approach (in our case an } \\
\text { MM)? }\end{array}$ & $\begin{array}{l}\text { Focus group, case studies } \\
\text { and literature review }\end{array}$ \\
\hline Is the improved approach valid and useful? & $\begin{array}{l}\text { Industrial trial/ } \\
\text { pilot assessment }\end{array}$ \\
\hline
\end{tabular}

\subsubsection{Literature review}

Literature review is one of the most important methods used to support evidencebased research [34]. In our research, we did semi-systematic literature reviews ${ }^{2}$ to identify, analyze and synthesize relevant (i) work related to our research (see Chapter 4), and (ii) work that could be useful to identify the relevant B-ITa processes in CNOs (see Chapter 7). Our first goal was to understand what others in our field of interest have successfully completed and how our work differs from theirs. Furthermore, we also wanted to demonstrate our understanding of relevant work summarizing such a work for the convenience of the ICoNOs MM.

"A systematic review is a means of identifying, evaluating and interpreting all available research relevant to a particular research question, or topic area, or phenomenon of interest" [34, p. 1052]. We searched for literature using several electronic indexing services (e.g., ACM Digital Library, Google Scholar, Citeseer library, and IEEExplore). When finding relevant papers, we traced the references in the identified papers to get access to other relevant sources. One can be argued that when selecting the papers for the literature review, we could make omission and inclusion errors [79]. Omission errors occur when a highly relevant

\footnotetext{
${ }^{2}$ They were semi-systematic literature reviews because we only followed partially the systematic review process presented by Brereton et al. [28]. We conducted the steps covered by the second phase of their process only.
} 


\section{CHAPTER 3. METHODOLOGY}

and reliable paper is left out of the review. For example, we could miss relevant work, for instance, because it is not written in English or even because it was not published or because at first sight the work had no relation with our research. Inclusion errors occur when an irrelevant or unreliable paper is included in the review. However, following what Aquino and Pagliarussi [79] suggest, we mitigated such errors by looking in (i) the degree to which a paper contributed significantly to our work, (ii) the way the papers introduced validated results and relevant conclusions of the work that they present, and (iii) the references in the identified papers to trace and get access to other relevant sources.

Levy and Ellis [179], quoting the work of Shaw [288], claim that locating relevant papers is certainly a necessary condition for literature review. However, it is not a sufficient condition. The information collected in the literature identified must be processed. We therefore reviewed the abstracts and the conclusions of the identified documents in order to determine their relevance to our research, and later we used interpretation (see Section 3.2.3) to analyze them.

\subsubsection{Focus group}

A focus group is a panel of professionals, led by a moderator and guided to exchange ideas, perceptions, and experiences on a specific topic [66]. Focus group research is a form of qualitative research. As such, it sacrifices reliability in favor of validity [307], i.e., focus group results cannot be replicated statistically, they are only useful in extracting data to validate findings. Though, focus group research is a widely popular method, due to its capability to generate a rich understanding of a topic by involving a group of people in an active discussion, rather than by using a single qualitative method, such as a survey [289]. A focus group session is commonly conducted with a group up to 12 professionals. It is therefore similar to small samples research [141].

Focus groups are best known for their usefulness to validate findings and gather recommendations that can derive changes from existing designs or new hypotheses [66, 289]. We used a focus group to start the validation of B-ITa domains to include in the ICoNOs MM. Similarly to Campbell et al. [41] who used a focus group to analyze strategic B-ITa, we decided to include this method in our research because of its fit to our research context and our purpose: (a) for MMs, people are valuable sources of information and a focus group is proven in provoking attendees to actively articulate their thoughts, perceptions, and behaviors [168]; (b) one of the MM authors can serve as the moderator to help attendees retrieve information; (c) the dynamics of a focus group is known to help generate valid and reliable data needed for the validation of artifacts in their early stage of development [212]; and (d) group interviewing is considered to be more effective than individual interviewing in particular research circumstances [124] which we also observed in our context. For example, when the research has general goals, "calls for qualitative data, requires data that is not in the respondent's topof-mind, and when there is minimal prior knowledge about a particular problem and the range of responses that are likely to emerge" [353, p. 167]. 


\subsection{RESEARCH APPROACH}

Focus groups can be contrasted to the Delphi method [222] where the anonymity of the participants and a general agreement between them as a whole are important. We decided to use the focus group method instead of the Delphi method because we were interested in the diversity of ideas and opinions of professionals working in areas related to our research, without attempting to build consensus. According to Larsor et al. [174], consensus does mitigate the divergent thinking which is a disadvantage of the Delphi method. We only wanted to collect attendees' perceptions, set in a 'permissive, nonthreatening environment' [171].

\subsubsection{Case study}

A case study is an empirical inquiry aimed at revealing aspects of contemporary phenomena inseparable from their real-life context, and thus, difficult to replicate in a laboratory environment. Case studies form one of the most used research methods for B-ITa issues ${ }^{3}$. "Case studies typically combine data collection methods such as archives, interviews, questionnaires, and observations. The evidence may be qualitative (e.g., words), quantitative (e.g., numbers), or both" [98, p. 534]. A case study can cover either single of multiple cases [348]. For example, Bleistein et al. [20] developed an integrated approach to requirements engineering for organizational IT alignment, and they illustrated its application and validated the approach by conducting one case study only. In contrast to this study, Earl et al. [96] studied four UK organizations to determine the relationships between business process reengineering, business strategy planning, and ISs planning; Hartung et al. [125] analyzed alignment levels in the Canadian Forces examining eight case sites; and Farrell [102] focused on the impact of IT in five organizations.

Based on the work of Dooley [91], Eisenhardt [98, 99] and Yin [348], case studies can be classified according to their application to research. They can be used to explain a specific situation, to provide description, to illustrate certain topics, to explore a specific situation, to validate approaches, and to test or generate theory. In the early stages of our research we used exploratory case studies because they are particularly appropriate for development and initial evaluation of new qualitative approaches as they allow the course of the study to be adjusted along the way to account for what is learned. Later, we used case studies to validate several components of our ICoNOs MM (see Chapters 5, 6 and 7).

In addition, our main motivation for using the case study method rests on the following:

(a) case study research involves multiple sources of data collected in a structured way which suggests that construct validity must be addressed, and that we can generalize results to valid statements [348];

(b) case studies are also the most suitable approach when there are many variables of interest and few data points and where resources do not permit enough replications to isolate the variables individually;

${ }^{3}$ See the IT alignment bibliography collected by Chan and Reich [51]. 


\section{CHAPTER 3. METHODOLOGY}

(c) case study research helps to analyze complex phenomena in depth in a reallife context rather than in isolation [15, 348], and this is exactly the context of the B-ITa criteria of interest in our MM.

\subsubsection{Industrial trial/pilot assessment}

Similarly to the validation attempts conducted by April et al. [5] to test their software maintenance MM, we used an industrial trial to validate the usability of the ICoNOs MM. Industrial trails, or pilot assessments, are commonly used to find deficiencies or problems in the design of a proposed work. These can be addressed before time and resources are expended in deployment, and later in the maintenance, of an MM (see Section 4.2.1 in Chapter 4).

Sponsorship from a CNO was necessary in order to use a final prototype of our model to appraise the maturity of its B-ITa. After the assessment, we asked to professionals of the appraised organization how they perceived the assessment process, the model, and the results. With such information, we could evaluate the usability of the ICoNOs MM.

\subsubsection{Data-gathering techniques}

In the previous paragraphs, we have described the research methods we used for the development of our model presenting the rational for choosing them among other ones. In this section, we introduce the techniques that we used for collecting data when conducting the case studies presented in Chapters 5, 6 and 7, specifically. We collected data in accordance with the hermeneutic approach. Hermeneutics claims that we can understand a complex whole from preconceptions about the meanings of its parts and their interrelationships [165]. In our particular case, hermeneutics helped us to obtain results from analyzing the information obtained using the different data-gathering techniques we present in the following paragraphs.

\subsubsection{Semi-structured interviews}

Semi-structured interviews are the most widely used data-gathering technique in qualitative research [32]. This kind of interviews is commonly based on a set of predetermined questions. However, other questions can come out from the dialogue between the interviewer and the interviewee. "The semi structured interview... recognizes that by offering respondents a chance to elaborate on their fixed-choice answers that both hard, comparable and rich, meaningful data can ensue" [228, p. 299]. That is, in semi-structured interviews the interviewee participates in meaning making and not only as a means by which data is retrieved.

The interviews that we conducted in our research were theory-driven interviews [228]. We started each interview by presenting the overall definitional foundations and context of our research to each interviewee. The goal was to make the interviewee understand our research context and concepts. With such 


\subsection{RESEARCH APPROACH}

an understanding, the interviewees had the opportunity to explain and clarify their own thinking. In this context, the interviewee could provide more meaningful answers to our questions, leading to new questions that were useful to clarify our own ideas.

\subsubsection{Observation}

There are several definitions of observation $[107,119,226]$. The common denominator among all the definitions is the need to study and understand people within their natural environment. For example, according to Gorman and Clayton [119, p. 40], observation studies are those that "involve the systematic recording of observable phenomena or behavior in a natural setting". The value of observation as data-gathering technique is that it allows researchers to study people in their home grounds in order to understand issues from their perspective [11, 107].

We decided to use observation in our research because we wanted to record situations as they occur and because for us it was important to analyze the environment of the case study sites since we incorporated a realist approach in the interpretation of the collected data (see Section 3.2.3). With observation it could be possible to identify issues and actions that people do not report because they think that such issues and actions are irrelevant [240]. In the observations, we played the role of 'complete observer' [11]. That is, we played a passive role being present in several meetings without interacting with people. Our role involved just two actions: to hear and to observe.

\subsubsection{Documents analysis}

No matter how unnoticeable a researcher wants to be in the interviews or in the observations of events to collect data, the researcher is always going to have an impact on the natural course of situations. One technique that helps to avoid this situation is the analysis of documents because documents do not react to the researcher's participation [226].

Document are commonly supplementary sources of data in qualitative research [107]. In our research, documents were an important data source. Documents are not simply containers of meanings. They are collectively produced, exchanged, and consumed. They summarize many decisions made by more than one person for a specific purpose. Documents represent specific circumstances including different insights. Therefore, when analyzing documents, it was important for us to know who had produced the document, for which purpose and for whom. This helped us to incorporate the context of the documents in the interpretation of data.

\subsubsection{Data analysis techniques}

The methods used to analyze the data collected by using the three techniques presented in the previous section (i.e., interviews, observation and analysis of documents) are presented in this section. 


\section{CHAPTER 3. METHODOLOGY}

\subsubsection{Interpretation}

Data analysis was primarily conducted using interpretation [165]. By using interpretation a researcher "does not predefine dependent and independent variables, but focuses on the complexity of human sense making as the situation emerges; it attempts to understand phenomena through the meanings that people assign to them" $[165$, p. 69$]$. In our research, interpretation helped us to create an understanding of the context of B-ITa projects, and the specific processes whereby B-ITa influences and was influenced by such a context.

The matter that we did the data analysis by ourselves clearly reflects some bias in how we interpreted the data. However, we motivated our decision to use interpretation by the following statements: first, documentation was an important data source in this case study. As we have stated in the previous section, documents do not only contain meanings, but they represent particular facts attending events and having some bearing on the meanings including different insights. Therefore, the analysis of documents required interpretation [106]. Second, people were data sources when conducting the interviews. In such a situation, interpretation also is a suitable analysis technique. Generally, people develop and use their own understanding and observations. Therefore, it was expected that the interviewees attached their own meanings to their answers in the interviews. People interpret their world and we, as observing researchers, interpreted their interpretations. Third, we believe that the interpretation technique can provide insight to identify the external forces and processes that constitute the means through CNO events unfold [227].

\subsubsection{Hermeneutics}

Hermeneutics is a major branch in interpretative studies [165] where organizational documents and documents derived from interviews are an important source of data $[243,330]$. Hermeneutics support the creation of understanding from the analysis and interpretation of data since it is focused on studying a whole by analyzing its individual parts and their combinations [111, 165, 330]. "The process of interpretation moves from a precursory understanding of the parts to the whole and from a global understanding of the whole context back to an improved understanding of each part" [165, p. 71]. This analysis technique has been used by IS researchers since the 80 's [24] for different purposes in a variety of topics ${ }^{4}$.

The reason that led us to recognize the use of hermeneutics in our analysis process was that this technique is a recognized method for analyzing organizations within socio-technical and organizational contexts [330], i.e., contexts where an interaction between people and technology within organizations exists. And this is the context where B-ITa projects can be found.

${ }^{4}$ Please refer to Webb and Pollard [330] for a list of different IS studies that use hermeneutics as analysis technique. 


\subsection{VALIDITY OF THE RESEARCH}

\subsubsection{Realist approach}

The analysis of data using interpretation from an hermeneutics perspective was strengthened by following the realist approach of Pawson and Tilley [230]. The realist approach is commonly used for evaluation and not for analysis. A realistic evaluation starts from the description of the logic of a situation, where an action is causal only if its outcome $(\mathrm{O})$ is triggered by a mechanism $(\mathrm{M})$ acting in a given context (C). Pawson and Tilley call this situation as a 'CMO configuration'. These configurations must work according to an initial theory. Applied in a different context $\left(\mathrm{C}^{\prime}\right)$, the same mechanism $(\mathrm{M})$ will produce expected - and unexpected or even adverse - effects (O'). The analysis of these effects and their relation to the context leads to a refined theory $(\mathrm{T})$. By using different contexts, one can produce a reasonably good understanding of the mechanism at work that later could be useful for generalizations. However, besides evaluation, the realist approach can also be used for analysis of data. For example, Crinson [69] used this approach to analyze qualitative data obtained from a focus group, and Davis and Wright [78] analyzed a continuous improvement process by taking a realist perspective.

In our research, we take the concepts of Pawson and Tilley's work (i.e., mechanism, context and outcome) and apply them in the analysis of data. That is, the realist approach helped us to identify underlying mechanisms for what we observed and to relate this to the B-ITa domains in the context of the case studies we conducted (see Chapter 6). Specifically, we synthesize findings and draw conclusions by following the realist synthesis method. According to Pawson et al. [229], a realist synthesis is a method for reviewing research findings related to complex social situations. It helps to provide an explanatory analysis of how and why something works (or does not work) in particular contexts. When analyzing the data, we then had a good understanding of the B-ITa domains and processes (this is what Pawson and Tilley would call the 'theory') in order to use the data for refining them. We did this having in mind that a situation is never the same, and never has the same impact, in different organizations because differences in context, type of organization, stakeholders and outcomes.

\subsection{Validity of the research}

In the previous section, we have presented the research methods and the techniques we used to gather and to analyze data. We make the note that all these methodological choices have consequences for the validity of our research. In this section, we introduce the concepts of construct, internal and external validity, and we describe how we addressed the threats to validity of each type following the strategies presented in Figure 3.2.

\subsubsection{Construct validity}

"Construct validity involves making inferences from the sampling particulars of a study to the higher-order construct they represent" [285, p. 65]. It is the degree 


\section{CHAPTER 3. METHODOLOGY}

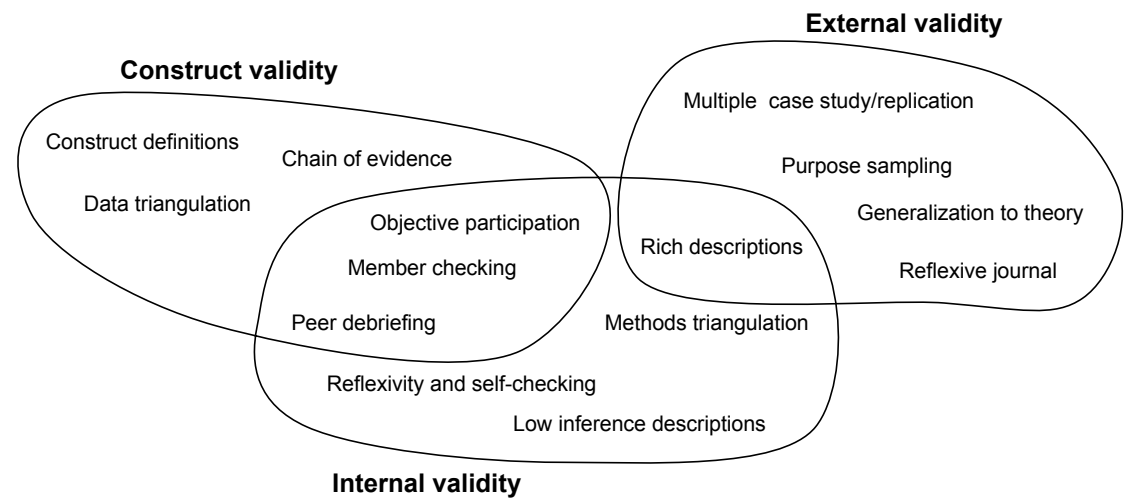

Figure 3.2: Strategies used to promote validity in our research.

to which evidence found in a study matches its theoretical definition. Therefore, it is important to define in detail the concepts of interest clarifying what one means with each of the concepts [61,285], and assess the match between the particular study and such concepts [285].

The conceptual research conducted to present our definitional foundations in Chapter 2, and the two literature reviews carried out in the problem investigation and the solution design phases of our research (see Figure 3.1), were steps to assure construct validity. We grounded the definitions (i) of the main three concepts of the research presented in this dissertation (i.e., B-ITa, CNOs and MMs) and (ii) of the components of the ICoNOs MM in a wide literature search which helped us to build our own conceptualizations of these concepts and components (construct definitions strategy in Figure 3.2). Moreover, in order to assure the match between the projects of the CNOs we studied and our research's concepts/interests, we met several times with professionals from such CNOs in order to guarantee that they had a B-ITa project in a CNO context. This was done before each case study started.

Another validity concern is that "case study research generally is perceived as subjective because researchers usually have close and direct personal contact with organizations and people examined. Hence, researchers need to make efforts to refrain from subjective judgments during the periods of research design and data collection to enhance construct validity" [255]. Following the recommendations of Yin [348] for ensuring construct validity, in the case studies that we conducted to develop the ICoNOs MM (see Chapters 5, 6 and 7), we (i) used data triangulation in the data collection phase, i.e., we used professionals, meetings, documents and illustrative materials in order to avoid researcher bias; (ii) established a chain of evidence, i.e., we used verbatim interview transcripts and notes of observations in order to trace research process steps and allow cross checks of particular sources of data; and (iii) let professionals - from the studied CNOs - and researchers review the data analysis and the final report outlining the findings (member checking and peer debriefing strategies [68] in Figure 3.2, respectively) in order to identify 


\subsection{VALIDITY OF THE RESEARCH}

and change unclear aspects.

Furthermore, construct validity in a focus group is concerned with whether (i) the focus group is actually discussing and evaluating what it is trying to discuss or evaluate [215], and (ii) the researchers interpret feedback from the focus group appropriately. In a focus group, the researchers and the participants are the instruments to 'measure' a specific work. The data that is obtained by using a focus group are the comments and recommendations given by the participants. This data is construct-valid to the extent that it is free from external influences (e.g., criteria of the researcher who is playing the role of moderator, or influences from the environment). In the focus group we conducted, after presenting the work to be evaluated, we tried to avoid conveying our meanings on specific aspects of our work, i.e., our participation as moderators was objective (objective participation strategy in Figure 3.2). Then, in the feedback analysis phase, we dealt with questions as: Are we correctly relating the feedback to specific concepts? Did the focus group participants actually mean what we think they meant, or they meant something else? Morgan [212] and Krueger and Casey [171] state that when the researcher is from the same background as the focus group participants, these last two validity threats are reduced.

\subsubsection{Internal validity}

Internal validity refers to the validity of an inference that a relationship between two variables is causal. This is commonly explained and exemplified for quantitative research only $[285,345]$. However, qualitative research also undergoes internal validity but not necessarily with the same interpretations [345], i.e., it is not exclusively involved with issues of cause and effect. Instead, internal validity in qualitative research relates to the quality [282, 294], rigor [74] and trustworthiness $[135,180]$ of data such that it assures that the results of the research are caused by the phenomena under investigation and not by incidental influences. In qualitative research, internal validity is therefore affected by qualitative research designs and it has to be addressed in the process of analysis because qualitative research expects to arrive at good interpretations of the phenomena under study [67]. We addressed internal validity by:

- reflexivity and self-checking, i.e., the continuous evaluation of the - subjective - data obtained and the research process itself [105, 247],

- peer debriefing, i.e., the review of the data and research methodology by someone who is familiar with the research [68, 224],

- low inference/rich descriptions, i.e., the use of - almost literal - descriptions that are very closed to interviewees' answers and/or researcher's notes and observations, e.g. verbatims [151], to present the setting, the participants, and the themes of a qualitative study in rich detail [68], 


\section{CHAPTER 3. METHODOLOGY}

- member checking, i.e., the examination of data and interpretations by the participants in the study - e.g. interviewees - to confirm the credibility of the information and narrative account [68, p. 127], and

- methods triangulation, i.e., the use of more than one method of research in a single research study [151].

Because our (interdisciplinary) research required knowledge from different disciplines, it was necessary to combine several insights in order to generate our own knowledge. We conducted conceptual research (literature review) for this purpose. However, as we are not experts in all the disciplines involved in our research, we could clearly have made omission and inclusion errors (primarily because of intrinsic personal bias or bounded rationality [79]) althought we made efforts to mitigate them (see Section 3.2.1). The review of literature surely had impact on the internal validity. Aquino and Pagliarussi [79, p. 13] claim that "the researcher must substantiate his or her chosen set of works. It is necessary to disclose the specific procedures used when building the review". We therefore explain what we exactly did in our reviews in order to mitigate internal validity threats (see Chapter 4). Moreover, specifically in the case studies, we consistently tried to avoid imposing (our) external meanings in the interpretations of the findings. We dealt with questions as: Is what we observe/hear what we think we observe/hear? Is our interpretation of the data true? Does this apply to other B-ITa projects or CNOs? Was this topic raised in all the interviews? How can our knowledge and position influence the analysis of data? Hermeneutics helped us to mitigate these threats. In a hermeneutic approach, the researchers acknowledge and make explicit how their understanding changed over time when reconsidering and reviewing emergent interpretations [330].

The three methods that we have used to collect data in the case studies (i.e., interviews, observation, and document analysis) are the most used data-gathering techniques in qualitative research [226]. This leads us to think that they provide significant advantages to research when collecting the 'right' data. For example, by interviewing, researchers can get very specific answers; by observing, researchers have the opportunity to see people in their natural environment; and by analyzing documents, researchers can deeply study information from different views. However, we acknowledge these methods also have disadvantages. Although interviews help to get the 'best' answers from the 'right' persons, how can we assure that we interview those right persons? We want to make explicit that, in our research, choosing the interviewees could have been a subjective task, and that it may be possible that we could get different information if we interviewed different people. Furthermore, there are several technical issues with the interviews that could interfere with the findings. We recorded the interviews with a tape recorder. Although we had the opportunity to produce high quality taperecordings, several of the tape-recordings had excessive background noise. This created difficulties in the research process when we transcribed the interviews. These transcripts could not be accurate because omissions or mistaking words that could change the meaning of ideas or specific sentences. Observations also 


\subsection{VALIDITY OF THE RESEARCH}

have a similar problem: how can we assure that we are observing the right event? In addition, observation could be affected by the time when we conducted the observations; and because observation is entirely based on our own participation, we could miss something that was important to our research.

Finally, in the focus group we strengthened internal validity by providing an appropriate amount of guidance to the participants without involving or introducing any of our own opinions in the discussions (objective participation strategy in Figure 3.2); and in the industrial trial/pilot assessment we considered questions as: Does our ICoNOs MM actually assess what we wish it assesses? Are the assessment results attributable to B-ITa processes in the CNO studied, or to some other related issues? How can our participation in the industrial trial affect the assessment results?

\subsubsection{External validity}

External validity pertains to how generalizable the results of the research are, i.e., it is concerned with the judgment whether a study's results can be generalized to other people, settings, and times [285]. In qualitative research, external validity is a matter of the fit between the situation that have been studied and others to which the researcher might be interested in applying the concepts and conclusions of that specific study.

Because in our research we conducted interpretative case studies, it can be argued that we cannot generalize results at all. To confront this validity concern, we based our analysis process on theories, frameworks, and principles developed by case study research methodologists - e.g. [165, 175, 325, 348]. In summary, they claim that in interpretative research the generalization from empirical descriptions to theoretical statements is possible and valid. So, in our case the empirical descriptions specify the description of the ICoNOs MM and its B-ITa process areas, and the details of the case study sites. The resulting theoretical statements could comprise a theory explaining the environment of the case study sites, structure and/or specific characteristics that would account for the performance of the B-ITa process areas found in the sites themselves (generalization to theory in Figure 3.2). Furthermore, since we conducted a multiple case study $^{5}$ to validate part of the ICoNOs MM (see Chapter 6), part of our results can at least be directly generalizable [348]. External validity, in this case, was addressed by replicating findings and confirmatory data analyses [224] (multiple case study/replication strategy in Figure 3.2). From the realist approach, this is what Pawson and Tilley [230] term 'cumulation', i.e., we replicated findings by a series of follow-up studies, self-consciously linked that progressively helped us to refine our understanding about the mechanisms, contexts and outcome patterns in our research. We moved from one case to another, not because they were similar, but because we had ideas that could encompass them [230, p. 119]. What

\footnotetext{
${ }^{5} \mathrm{~A}$ multiple case study differs from a set of single case studies in that a multiple case study is an empirical inquiry that investigates a phenomenon within its real-life context to answer the same research question in different single case study sites.
} 


\section{CHAPTER 3. METHODOLOGY}

was transferable between cases was not data but ideas that became strong by cumulation.

Shadish et al. [285] state that random selection of participants assures a representative sample of a target population, and therefore findings are generalizable. However, they also claim that random sampling is rarely feasible in field studies. In the case studies we conducted, the sites were not systematically sampled. Therefore it can be claimed that we cannot generalize findings to a wider population of organizations. However, we used 'purposive sampling' [181] to select the case study sites that were diverse on specific characteristics. When conducting the case studies to validate the B-ITa domains (see upper side of the solution design layer in Figure 3.1), we made sure to study different case study sites. We chose CNOs from different countries, one international and three of national nature, one entrepreneur-led and three government agencies, and two with a large amount of participants and two with only 2 or 3 participating organizations. We must also note that the B-ITa key drivers they have are different. The key drivers of one of the studied CNOs are to control costs and to manage risk, while the B-ITa key drivers of the other sites are to improve quality and to increase effectiveness. Our aim was to obtain final results that could be shared and applied beyond the study setting of each site. Furthermore, when reporting the case study findings, we provided broad explanations of contexts in order to inform about our decisions concerning to whom the results may be generalized.

Finally, we want to make the note that while doing research, we maintained a (no detailed but still meaningfully) reflexive journal [181] to document thinking, impressions and observations mostly during the data collection process (e.g., after each interview was conducted in the case studies). This journal helped us to look at the practical side of our work and at several issues that are associated with it from a more holistic point of view. According to Lincoln and Guba [181] reflexive journals also can help to strengthen external validity.

In this section we have so far presented several threats to our research validity and we explained how we address them. We recognize that each of the methods and techniques we used in our research has more validity threats that need to be considered. Such threats and more validity issues are presented in the next chapters because they are more specific to the studies we conducted.

\subsection{Summary}

This chapter presented the method we followed to develop the ICoNOs MM, the techniques we used to collect and analyze data in our research, and several validaty threats and issues that could have an impact on our results.

The methodology comprises three main phases. Phase 1 dealt with the investigation of the problem, and phases 2 and 3 addressed the design and the validation of the solution, respectively. We have not presented details of the steps that each of these phases involved. These detailed explanations can be 


\subsection{SUMMARY}

found in the following chapters of this dissertation. Specifically, the following chapter deals with phase 1 by describing the literature review that we conducted to identify advantages and disadvantages of current B-ITa assessment approaches; Chapters 5, 6 and 7 pick up phase 2 of this methodology and describe the focus group, literature review and case studies that we conducted to design the ICoNOs MM; and finally, the phase 3 is addressed in Part III of this dissertation.

We have also described the research methods (literature review, focus group, case study, and industrial trial/pilot assessment), data-gathering techniques (semistructured interviews, observation, and documents analysis), and data analysis techniques (interpretation, hermeneutics, and realist approach) we used in our research. Our aim was to present the rational for their inclusion in our investigation. We make the explicit note that the main method used in our research is the case study. The reason for this is that we wanted to investigate phenomena in a real-life context rather than in isolation in order to develop not only a state-of-art literature-based MM, but also a model that reflected the actual real environment and work of CNOs. This, in turn, also helped us to validate the components of the model assuring a good solution design.

Finally, we explained how we reduced the possible threats to construct, internal and external validity. These threats cover issues from the interdisciplinary nature of our investigation to several disadvantages of the data-gathering techniques and research methods we used. According to Lincoln and Guba [181], presenting validaty threats in an explicit manner enhances the validity of the research method itself. 



\section{4 \\ Related work}

As already indicated in Chapter 1, there have been some proposals for assessing the alignment between IT and business. However, as these proposals are oriented to single organizations, they fail to take specific characteristics of CNOs into account, such as the need for coordination, the lack of centralized decision making or the heterogeneity of IS architectures. Besides such proposals, there are also models that can be used to assess the maturity of particular different aspects within CNOs. However, to the best of our knowledge, at the time of writing this dissertation, there is no a single model that addresses all relevant aspects to assess B-ITa in a CNO context. In this chapter, we address our first research question (see Section 1.2.2 in Chapter 1):

\section{Q1 What are the current assessment approaches of B-ITa?}

First, we take a look at B-ITa MMs in Section 4.1 and we make a comparison of them in order to identify their advantages and shortcomings (Section 4.2). This serves to define the improvement goals and reusable knowledge to address and use in the development of the ICoNOs MM, respectively. Furthermore, we present an overview of several MMs for CNOs in Section 4.3 in order to define what is important to include in our model to cover a collaborative perspective.

We did a semi-systematic literature review (see Section 3.2.1) to select the related work presented in this chapter. The performed literature review consisted of a broad search of academic and practitioners' information sources. We approached the literature search using several electronic indexing services (e.g., ACM Digital Library, Google Scholar, Citeseer library, and IEEExplore). A set of key words was used: alignment, business IT alignment, strategic alignment, IT alignment, architecture alignment, maturity model, assessment tool, measurement guide, networked organization, business network, collaborative enterprises. We also used some alternative terms for alignment when identifying the B-ITa MMs: balance, harmony, fit, and linkage. We traced the references in the identified papers to get access to other relevant sources. We reviewed the abstracts and the conclusions of the identified documents in order to determine their relevance to our research. 


\subsection{Assessment approaches of B-ITa}

In this section, we present MMs that have been developed for assessing B-ITa. The common denominator among these assessment approaches is that they cannot be used to conduct assessments in a CNO level. They are thus oriented to single organizations.

\subsubsection{Luftman's MM}

The model of Luftman [189] has been developed based on his practical experience and research on the enablers and inhibitors of alignment [191], the strategic alignment model [130], the CMM, the reach and range concept of Keen [157], and Nolan's stages of growth model [220]. Luftman's MM is an approach to determine a single organization's B-ITa based on six domains. These six domains are skills, technology scope, partnership, governance, competency measurements, as well as communications. We briefly describe these domains in the following:

- Skills involves human resources concerns such as cross-training in IT and business issues and the cultural environment towards organizational change and innovation.

- Technology scope refers to the extend to which IT provides a clear and flexible infrastructure, and implements emerging technologies and IT standards.

- Partnership centers on how the IT and business sides in an organization perceive the contribution of each other in order to manage their relationship properly.

- Governance pertains to the appropriate review, analysis and prioritization of IT resources (software, hardware, ect.) to support the activities in the organization.

- Competency and value measurement refers to how the contribution of the IT side to the business side is valued.

- Communication entails knowledge sharing and understanding for ensuring successful business and IT strategies.

Each of these domains is assigned the next five levels of alignment:

1. Initial/ad hoc is the lowest process level of alignment where IT and business are not aligned and the processes to improve such an alignment are ad hoc in nature.

2. Committed process level pertains to the existence of commitment by the organization to promote a global B-ITa vision. 


\subsection{ASSESSMENT APPROACHES OF B-ITA}

3. Established focused process level relates to the existence of an established B-ITa process that is focused on business goals.

4. Improved/managed process level refers to the use of a well-founded B-ITa process that emphasizes the concept of technology as value creator for the organization.

5. Optimized is the highest process level of alignment and refers to the strategic B-ITa process that is fully integrated and co-adaptive between the IT and business sides.

The level of alignment for each individual domain is determined by means of answers to several questions. However, notwithstanding the effective use of this well-known MM, the model disregards explicit interrelations among the domains that explain B-ITa and it is focused to single organizations.

\subsubsection{CIO Council's assessment guide}

The Chief Information Officer (CIO) Council, a consortium of CIOs of US federal executive agencies, developed an architecture-specific alignment and assessment guide [103]. This guide provides an overview of the integration of enterprise architecture concerns into the information technology investment planning process. Specifically, this guide is useful to relate capital investments to the architecture project assessment and IT capital planning functions. It is intended for the senior management as well as for the architecture practitioner. Its main focus lies on presenting a step-by-step architecture alignment and assessment process using several tools. Some of these tools are:

- Business alignment tool that indicates where a relationship exists between a project concept/requirement and an organizational objective by identifying points of interaction in a two-dimensional matrix. To extend the results, this tool uses a business scorecard to include subjective alignment scores $(-3$ to +3$)$ to describe the interaction points.

- Business case worksheet that organizes the strategic, technical, and financial information required to make an investment decision in a consistent, structured format. The worksheet provides sufficient information for the investment and architectural evaluators in the organization to evaluate and rank a project against other proposed investments according to the project benefits, costs, risks, and architecture criteria.

- Technical alignment matrix that provides a framework for determining "whether the technology architecture of the proposed solution is in compliance with the enterprise standards, architecture (i.e., information, data, applications, knowledge, and technology), and methodology (i.e., software development, data definitions, and network design)" [103, p. 22] as defined by the technical reference model. 
- Architecture assessment matrix that provides a framework for determining if a specific project's documentation is in compliance with the architecture components approved through the technical alignment process.

In summary, the CIO Council's assessment guide is useful to determine the degree to which a proposed investment aligns with business strategies, and to know how well the design of investments aligns with the infrastructure architecture. This assessment model is primarily focused on investment studies in federal agencies. It identifies no specific B-ITa domains, and thus it provides no support to identify opportunities of improvement in organizations on particular areas.

\subsubsection{Duffy's MM}

The MM developed by Duffy [95] is based on the premise that a reliable partnership between IT and non-IT executives is fundamental for achieving a successful B-ITa. Duffy recognizes that IT and business objectives are interdependent, and therefore, a division of practices into IT and non-IT areas would generally be unfavorable. This model is structured around series of key success drivers (domains) which are operationalized in key performance indicators (KPIs) containing five contributing factors each. Duffy explains that a contributing factor is an aspect that is designed to address a specific and critical question in the KPI where it is included. According to Duffy, the six domains required to understand B-ITa are:

- Human resources organization and management. This domain refers to the workforce of an organization. The model emphasize the importance of the ability to attract, retain and manage such a workforce.

- Innovation and renewal strategy. This domain centers on how innovative an organization is and what processes the organization must perform to ensure that its knowledge and capabilities do not turn obsolete.

- IT/business architecture. This domain refers to the entities involved (i) in the business environment of an organization (e.g., customers, suppliers, competitors, etc.) and how they interrelate - i.e., the business architecture, and (ii) in the information and applications - i.e., IT architecture, that support the processes and such a business architecture.

- IT/business partnership. This domain pertains to how the role of the IT function in an organization has changed from a strict support function to a full strategic business partner. "Technology is critical to business success and this co-dependency drives the need for the IT and non-IT executives to pursue a win/win relationship" [95, p. 4].

- Operational excellence. This domain centers on the performance of the organization. Duffy recognizes that operational excellence can only be achieved if the organization (i) recognize the value of issues as learning and partnerships, and (ii) can response to market's demands on time. 


\subsection{ASSESSMENT APPROACHES OF B-ITA}

- ROI strategy and management. This domain refers to the metrics and processes required to manage the finance of the organizations without isolating the IT cost and benefits, i.e., the IT contributions must be taken in consideration together with the business ones.

Although the six domains reflect the "IT and non-IT" premise and several strategic issues, the model does not have explicit maturity levels for each of the domains. Instead, Duffy combines the six domains figuring out four B-ITa scenarios where organizations can be categorized: "uneasy alliance", "supplier/consumer relationship", "co-dependence/grudging respect", and "united we succeed, divided we fail". Such scenarios are the maturity levels in the model. Because there is not enough literature explaining the role of the IT/business architecture domain where the external business environment of the organization is addressed, we still cannot consider that Duffy's MM is a CNO-oriented model.

\subsection{4 de Koning et al.'s model}

Based on the analysis of business-IT excellence in several successful organizations in the Netherlands, and with the help of five hundred managers, de Koning and van der Marck [80] came up with ten questions that can be used to identify the level of alignment in single organizations. Those questions can be answered using a scale from 1 to 10 where the highest score means 'it entirely applies to my organization' and the lowest score means 'it entirely does not apply to my organization'. Although they do not identify B-ITa domains, this simple tool does cover several B-ITa-related topics. In their work [80], they have considered the following five guiding principles for achieving B-ITa:

1. Rationality is the guide in the IT decision making process. de Koning and van der Marck present how "no case, no go" (i.e., if there is no positive fact-based explanation of the reasons for investing in IT, no action should be taken) is the motto of the most successful businesses in the Netherlands for B-ITa-related decisions. It is to avoid unfavorable future results.

2. Business commitment in the IT projects must be organized. If the B-ITa vision is firmly-embedded through the strategic, tactical and operational levels in the organization, the relation between IT and business can be effectively maintained. In such a context B-ITa projects are commonly business-driven efforts, and not projects that are derived by not-grounded ideas.

3. IT efforts need to be controlled disciplinarily. Successful businesses in the Netherlands use business cases (where the impact on IT, processes and organization are evaluated) as basis for the IT decision making process. They spend extra time and money in clearly formulating starting points and boundary conditions before all project-related contingencies are taken into consideration. 


\section{CHAPTER 4. RELATED WORK}

4. IT competencies on the business side and business competencies on the IT side are important for positive B-ITa results. Business and non-business people must develop competencies in issues such as decision making, structure and change management.

5. IT projects must be approached from integral and partial perspectives. de Koning and van der Marck explain the dilemma between the integral and partial perspectives as follows: on the one hand, organizations must see IT as an integral component of changes in the entire organization. On the other hand, each change considered for implementation must be seen as an individual initiative in order to achieve/perform it successfully.

These principles were the basis for the development of a B-ITa maturity audit tool (BITAMA) available online at http://www.itzonderhoofdpijn.nl. BITAMA contains five pairs of propositions for each of the five principles. For each propositions pair, this tool presents two extremely opposite viewpoints. For example, "no guts, no glory" (i.e., because nothing is sure in this life, a promising idea must always be executed) vs. "no case, no go". By choosing what applies better to itself, an organization gets a score and a detailed explanation on how mature its B-ITa is (alignment level). BITAMA positions organizations in one of the following categories: on golden wings (business where both the business and IT performances are mature), millstone (business where both the business and IT performances are immature), heaven (organization with a successful business side only), and hell (organization with a successful IT side only). However, we make the note that the levels presented in the tools are limited to three: immature, puberty and mature. This model also overlooks the assessment of those processes that do actually help to achieve B-ITa.

\subsection{5 van der Raadt et al.'s MAAM}

The Multi-dimensional Assessment model for architecture Alignment and Maturity (MAAM) [312] can be used to assess architecture within organizations. The MAAM helps to identify the current situation of an organization's architecture, and to define improvement points and plans. The authors claim that a correlation exists between architecture alignment and architecture maturity. They claim that when architecture maturity increases, architecture alignment generally increases too (and when architecture alignment increases, architecture maturity also increases). The development of the MAAM started from the premise that an "organization where architecture awareness originates with business management has different ideas about architecture and faces different problems than an organization where architecture awareness starts in the IT-department" [313, p. 5]. To construct the model, van der Raadt et al. used variables of existing assessment models - e.g., [36, 189], theories from several research fields adopted to the IT domain - e.g., $[48,130]$, and their previous research on the aspects and critical success factors of architecture in practice [313]. 


\subsection{ASSESSMENT APPROACHES OF B-ITA}

This model visualizes architecture maturity and B-ITa on architectural issues. The MAAM can also be seen as a two-dimensional framework that relates architecture maturity on the first dimension with organizational alignment on the second dimension. The organizational alignment in this model helps to position an organization in one of the three following situations:

1. Organizations where architecture is imposed by the business management to give structure to the organization and to emphasize the business aspects of architecture.

2. Organizations where architecture starts at the IT department to structure the development process and the ISs it produces, focusing on the IT aspects of architecture.

3. Organizations where the focus of architecture lies evenly on the IT and business aspects, resulting in the alignment of business and IT goals.

The MAAM consists of the following six interrelated domains that explain the alignment and maturity of an architecture:

- architecture development process,

- architecture governance,

- organizational support for architecture activities,

- communication through and about architecture,

- organizational and logical scope of architecture,

- human and other architecture resources.

However, the model only assesses such an architecture by considering B-ITa as a stage that can be reached by increasing architecture maturity.

\subsubsection{CoBit}

COBIT, issued by the IT Governance Institute ${ }^{1}$, is a control framework that organizations can use for IT to be successful in delivering against business requirements by employing management best practices and measuring IT processes. "The business orientation of COBIT consists of linking business goals to IT goals, providing metrics and MMs to measure their achievement, and identifying the associated responsibilities of business and IT process owners" [144, p. 5]. Version 4.0 of this framework provides an explicit support to assess the alignment of IT with the business processes. For example, under the 'Defining a strategic IT plan' process, COBIT outlines how to engage IT managers on alignment with business goals and to develop a proactive process to quantify business requirements.

\footnotetext{
${ }^{1}$ More information on http://www.isaca.org/
} 


\section{CHAPTER 4. RELATED WORK}

COBIT supports B-ITa by focusing on the business' requirements for information, and the structured (process) utilization of IT resources. The framework has been based on the idea that IT resources must be managed and controlled on the basis of processes grouped in a natural manner. It contains 34 IT processes. For each of those processes, COBIT has a number of management instruments, such as:

- Control objectives, which are in fact the management objectives per IT process. The 34 processes together consist of 318 detailed control objectives.

- Critical success factors, which define the most important issues or actions for management to enable achieving control over and within its IT processes.

- Key goal indicators that, representing the process goal, are measurable indicators of "what" has to be accomplished in a process.

- KPIs, which define measures to determine "how well" the IT process is performing in enabling the goal to be reached.

- Maturity levels on a scale of 0 up to and including 5, where 0 stands for 'non-existence' and 5 stands for 'best practice'.

- Audit guidelines; directives for interview, information and tests which can be carried out to provide management assurance and/or advice for improvement.

The framework then utilizes a structured approach in describing each IT process in detail, i.e., it presents what business requirements the process is intended to fulfill, its focus area, how it can be achieved, and how it will be measured. The aim of COBIT is to support the management and the process owners by means of IT governance. The process owner should be able to determine the level of adherence to the control objectives either as a quick self-assessment or as a reference in conjunction with an independent review. Managers may wish to place any of these assessments in context by comparison to the industry and the environment they are in, or by comparison to where international standards and regulations are evolving (i.e., emerging future expectations). Although the organized configuration of COBIT, one of the problems with the framework is that it covers so many control objectives and management guidelines that it is challenging to make sense of them and to know on which of them organizations must spend efforts, and which of them can be ignored.

In summary, COBIT does establish what needs to be done providing the required information to achieve goals. However, (i) the focus of COBIT lies primarily on IT governance, (ii) it does not address a networked organization viewpoint, and (iii) COBIT deals with B-ITa from a strategic perspective. 


\subsection{ASSESSMENT APPROACHES OF B-ITA}

\subsection{7 van der Zee et al.'s assessment tool}

According to van der Zee et al. [314], the degree of alignment is mostly determined by the degree of maturity of changes in architecture. Managers must then look at the maturity of their organizations' architecture as start point for identifying improvement measures for B-ITa. The assessment tool developed by van der Zee et al. enables organizations to get aware of where they stand, what their competencies are, and which measures must be implemented to reach a higher level of maturity. The tool has five architecture levels of maturity grouped in three phases:

1 Reactive (the goals of the architecture-thinking paradigm are defined in terms of the results and the performed activities)

Level 1 "awareness": The IT and non-IT sides of the organization are aware of the importance of architecture.

Level 2 "formalization": The architect functions are formalized. However, architecture is just based on trust.

2 Proactive (the vision is clear and the architecture aims are reviewed in accordance with the organizational goals)

Level 3 "testing": The architects have clear-defined responsibilities. Their efforts are directed to specific areas.

Level 4 "management": The organization starts to work under architecture with a high degree of rationality vision.

3 High performance (the organization is aware of the strategic value of the implementation of architecture-based changes)

Level 5 "understanding": The architecture is seen as a component of the organization (business and IT) culture.

A key assumption embedded in this assessment tool is that an organization moves through the five levels in its way to optimally exploit its product and process architectures. The tool also elaborates on the roles of business/IT managers, architects, project managers, top managers and CIOs, for each of the architecture levels. According to van der Zee et al., these roles are important because it is the responsibility of these roles to recognize problems with the business or with the technology, and to find proper alignment of the two to solve these problems. With this model, it is possible to identify how organizations handle architecture and B-ITa. However, the maturity of B-ITa only is considered as a result of the maturity of an organization's architecture. 


\section{CHAPTER 4. RELATED WORK}

\subsubsection{Sanchez Ortiz's B-ITa instrument}

Sanchez Ortiz [265] developed an instrument to measure the level of alignment between business and IT strategic objectives. This B-ITa instrument consists of twenty questions that are based on the analysis of literature related to the domains that could compose B-ITa - e.g. [31, 37, 50, 70, 130, 155, 191, 262] - and the concept of total quality management. Specifically, the instrument attempts to measure B-ITa considering the areas of B-ITa uncovered by Chan [48], Henderson and Venkatraman [130], and the quality perspective of the MBNQA framework [246]. The questions in the instrument "were designed specifically to parallel some dimensions of the MBNQA:

- Leadership,

- Strategic Planning,

- Customer and Market Focus,

- Information and Analysis, and

- Human Resources,

but reflecting the fit between the priorities and activities of IT with those of the business" [265, p. 52]. For example, some questions are directed to assess items as the use of IT 'to achieve high quality performance', 'to communicate values and expectations', 'to support organizational and employee learning', 'to increase customer/citizen satisfaction', 'to determine current product/service requirements', and 'to promote cooperation, innovation, and flexibility'. To answer the questions, one can use a seven point likert-scale with $1=$ 'strongly disagree' to $7=$ 'strongly agree'. An option of Not Applicable (N/A) is also included.

Sanchez Ortiz's B-ITa instrument has been refined using the inputs of researchers on the areas of IT, management, and psychology; and has been validated by conducting a pilot assessment in the Denton City Government. However, it is also oriented to single organizations and, since the instrument is only a list of questions, it does not identify process areas which, as a consequence, limits that organizations plan improvements in specific B-ITa activities.

Heretofore, we have presented assessment approaches of B-ITa only. We found that all the models reviewed in this section have some aspect that can be improved. In the next section we compare these approaches in order to identify improvement goals to address, and reusable knowledge to use, when developing the ICoNOs MM. Then, in Section 4.3 we briefly introduce MMs that have do been developed for conducting assessments in CNO settings.

\subsection{Comparative analysis of the models}

In this section, we compare the B-ITa MMs presented in Section 4.1. The proposed framework for analysis and comparison is derived from the development 


\subsection{COMPARATIVE ANALYSIS OF THE MODELS}

process we followed to design our ICoNOs MM. Previous versions of this MM development process have been published in the proceedings of the 1st International Conference on Research Challenges on Information Science [271] and the International Workshop on Enterprise Interoperability [275]. In the following, we first introduce the development process (Section 4.2.1) before presenting the comparative analysis of the models in Section 4.2.2.

\subsubsection{MM development process}

Developing MMs systematically is not a topic that is widely covered in the literature. Instead, most of the MM literature presents the resulting models only and does not discuss the model developing process itself. The development of the ICoNOs MM consists of several steps (see Figure 4.1). Below we present detailed explanation of these steps. We make the note that because Figure 4.1 is a high level view of our MM development process, we excluded any discussion on feedback loops needed to keep the MM updated in a dynamic environment. We, however, acknowledge the importance of monitoring the MM and managing the evolution of CNOs when the MM is modified.

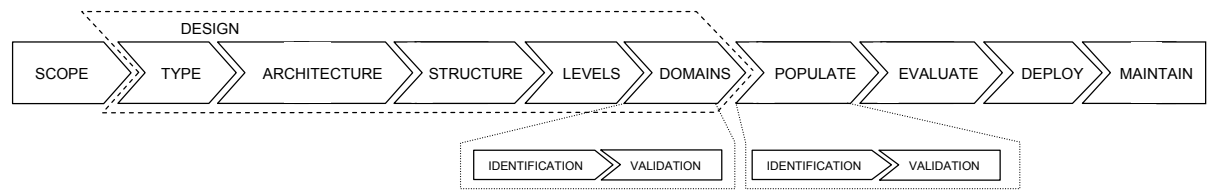

Figure 4.1: MM development process.

The first step in developing an MM is to determine the SCOPE of the model, which means to set the boundaries for the model's application and use, and to define the purpose of the model. This is to differentiate the model from existing MMs. The second step is to DESIGN the model. This step covers:

- the specification of the model's type.

Generally, MMs can be grouped in two types based on the key purpose these models serve when organization use them: assessment MMs and development MMs. The first category consists of normative models which serve as assessment tools - e.g. the SEI series of CMMI-compliant models (see Section 2.3.1 in Chapter 2). These models target certification, and help create or improve the organization's image as a reliable partner. The second type includes models serving as development tools that organizations use not because they strive for certification or status but because they need guidance and focus for implementing best practices and key process areas that, ultimately, lead to improvements and better results.

- the determination of the model's architecture. 


\section{CHAPTER 4. RELATED WORK}

The architecture of an MM describes the manner in which maturity levels can be reached. As we have already presented in Chapter 2, according to CMMI [54], 'staged' and 'continuous' are the two architectures that an MM may have.

- the organization of its structure.

The structure of an MM presents the organization of the components of the model. It defines if the MM does include domains, key process areas and goals, and how they are decomposed and used to reach maturity levels.

- the definition of the maturity levels.

This step implies to define the number of discrete levels of maturity for the model (typically five or six), and their qualifiers and definitions.

- the identification of the domains.

The last step related to the design of the model is the identification of the domains to which the levels apply. A domain is a relevant aspect within the scope of the MM. For example, CMMI for software development [57] recognizes four domains: process management, project management, engineering and support. This task is not simple because after identifying the domains, they need to be validated to assure that they correspond to the purpose of the MM.

Once the design of the model is completed, process areas need to be identified for each domain so that we POPULATE the model with observable domain assessment criteria. A process area is a group of practices in a domain which, when implemented collectively, satisfy goals considered important for making an improvement in that domain (e.g., a process area in the IS architecture domain is 'IS portfolio management').

After populating the model, it must be validated in order to EVALUATE its applicability and generalizability. The objective is to validate the entire MM to test it for relevance and rigor. Following population and evaluation, the MM must be made available for use to verify its generalizability (step DEPLOY). To provide its acceptance and to improve its standardization, the MM must be applied in organizations that differ from the organizations that were involved in its design and population. The identification of organizations that may use the model and the application of the model to multiple organizations are the final steps towards its spreading and acceptance.

Finally, it is important to track the evolution of (i) the organizational area or function that is assessed using the MM, and (ii) the requirements of the organizations that apply the model, in order to MAINTAIN the MM over time to keep it up-to-date. For example, the first MM developed by the SEI was the SW CMM. However, they observed that organizations would like to focus their improvement efforts not only in software engineering but also across different organizational 


\subsection{COMPARATIVE ANALYSIS OF THE MODELS}

functions. Therefore, the SEI came up with an integrated MM (CMMI) combining models from different disciplines to support the enterprise-wide process improvement that organizations were pursuing.

\subsubsection{B-ITa MMs comparison}

For the comparison framework we specifically focus on the DESIGN and POPULATE steps of our MM development process (see Figure 4.1). The final criteria that we use for the comparison can be found in the middle column of Table 4.1.

Table 4.1: Comparative analysis of B-ITa assessment approaches.

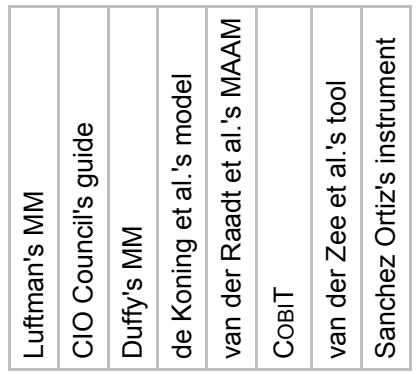

\begin{tabular}{|c|c|}
\hline \multicolumn{2}{|c|}{ SCOPE } \\
\hline \multirow{5}{*}{ 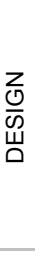 } & TYPE \\
\hline & ARCHITECTURE \\
\hline & STRUCTURE \\
\hline & LEVELS \\
\hline & DOMAINS \\
\hline \multicolumn{2}{|c|}{ POPULATE } \\
\hline \multicolumn{2}{|c|}{ EVALUATE } \\
\hline \multicolumn{2}{|c|}{ DEPLOY } \\
\hline \multicolumn{2}{|c|}{ MAINTAIN } \\
\hline
\end{tabular}
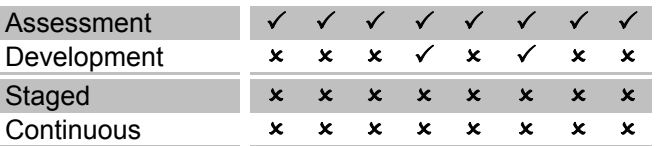

Num. of levels

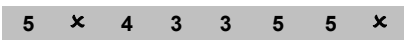

Domains Process areas

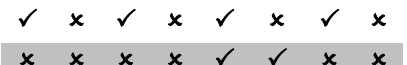

Alignment level

Orientation

Strategic Operationa

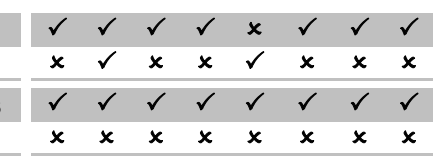

Legend: $\checkmark \quad$ The model considers the criterion

$x$ The model does not consider the criterion

We also include 'alignment level' and 'orientation' as criteria for comparing the models. These two criteria are added (i) to present whether the MMs are oriented to assess operational or non-operational B-ITa (see Section 2.1.3 in Chapter 2), and (ii) to indicate what we so far know about the MMs we are comparing, i.e., all models assess B-ITa in single organizations. 


\section{CHAPTER 4. RELATED WORK}

\subsubsection{Type of the model: Assessment vs. development}

All the seven models are assessment MMs. Only BITAMA and COBIT provide such considerable information on the assessment results and the process areas, respectively, that one could consider that both models are development MMs: they provide specific guidance to reach higher level of B-ITa maturity. The distinction between MMs as assessment tools and MMs as development tools is a discussion topic among MMs experts. Commonly, organizations choose an MM that not only enables them to determine the level at which they currently stand, but also provides them with guidance to reach a higher level of maturity. A development, i.e., assessment plus improvement, MM gives such a guidance. Based on our observations, we conclude that development MMs offer a more complete support to organizations. Similarly to BITAMA and COBIT, the ICoNOs MM is a development $\mathrm{MM}$ that implicitly provides an in-depth organizational transition plan for the CNO (including a roll up of recommendations, e.g., coordination mechanisms, implementation strategies and organizational changes) in the form of process areas, specific goals and practices (see Chapter 8). Through the levels of the ICoNOs MM, we intend to provide improvement routes for those factors that are most important for bridging the alignment gap in CNOs.

\subsubsection{Architecture of the model: Staged vs. continuous}

Architecture is a criterion that shows results which are remarkably distinctive from the results of the other criteria. Although several of the presented B-ITa MMs $[144,189,314]$ explicitly mention that the models are based on the CMM, none of the seven models discusses the architecture types used by CMM, i.e., staged or continuous. Only COBIT mentions that it is not designed to be a formal basis for certification with discrete levels that create thresholds that are difficult to cross. That is, when using COBIT there is no number for which to strive. It has been designed to provide descriptions that organizations can recognize as best fitting specific processes.

In CNO settings, the ICoNOs MM is a continuous MM. We find this representation more suitable because a $\mathrm{CNO}$ can have different levels of maturity in each of the domains included in the model. A continuous model will let the CNO focus, for instance, on the domains with a low level of maturity. Those domains that are associated with higher maturity can, then, be candidates for inclusion in later improvements efforts. To illustrate this, imagine the management of an organization wants to improve (i) how the organization controls the ISs and (ii) the allocation of decision rights and responsibilities, i.e., IS architecture and partnering structure in our model (see Chapter 8). The organization is satisfied with how the organization's other areas are developed, i.e., process architecture and coordination, and so decides to focus on the IS architecture and partnering structure domains. With our continuous MM, this organization will concentrate on only those domains trying to improve processes related to them to reach a higher level of maturity on those areas. If the organization successfully achieves what is 


\subsection{COMPARATIVE ANALYSIS OF THE MODELS}

described in level 3 for those domains (suppose it had level 1 in both units), it could be said that the organization is at level 3 in IS architecture and partnering structure; and maybe the organization still has lower levels of maturity in other domains, or otherwise. A continuous B-ITa MM for CNOs helps to a) obtain a more granular assessment for each domain, and b) to identify a specific practice across the maturity levels to find its path from a low level of maturity to a higher one.

\subsubsection{Structure of the model}

In Table 4.1, we do not include 'structure' as a criterion for comparison since each of the models has its own structure and fashion to relate its components to each other. Nevertheless, we do discuss the structure of the ICoNOs MM in turn.

The structure of the ICoNOs MM is based on CMMI-DEV [57]. Some CMMI design choices are also present in the ICoNOs MM. This situation avoids starting with the development of the model from scratch, and, most important, it also prevents our future users from starting over when adopting our MM for their B-ITa assessments.

The ICoNOs MM has four layers of aggregation (see Figure 4.2). The upper layer consists of the domains that must be addressed in a $\mathrm{CNO}$ when achieving B-ITa (see Chapters 5 and 6). The next three layers reflect the overall CMMI structure $[57$, p. 30]. In each of the domains we can find process areas. Process areas are sets of activities that are performed to make improvements in a particular domain (see Chapter 7). Similarly to CMMI, the ICoNOs MM process areas have specific and generic goals, which the activities in the process area are supposed to achieve. Specific goals describe characteristics that must be present to satisfy a particular process area; they are specific for this area. Generic goals apply to all process areas, although their instantiation for each process area can differ. For example, a CMMI generic goal is 'the process is institutionalized as a defined process'. Our MM incorporates the generic goals of $\mathrm{CMMI}^{2}$. The goals are decomposed in specific and generic practices describing what a CNO may implement to achieve the specific and generic goals. These practices are expected and not mandatory. This means that it is permitted to implement alternative practices in substitution for the specific and generic practices that the ICoNOs MM includes. The only condition is that the goals must be satisfied, to perform a process which is required for an organization to reach a specific maturity level.

\subsubsection{Levels in the model}

Because we are analyzing MMs, it was expected that all seven models include levels. Some of the models explicitly take their levels from the CMMI. Those models that did not, have designed their own levels. To maintain adherence to the CMMI, which is an internationally well-known MM, we decided to base the levels of the ICoNOs MM on the CMMI, too. Since our MM is a continuous MM

${ }^{2} \mathrm{~A}$ detailed list of these generic goals can be found in Appendix B. 


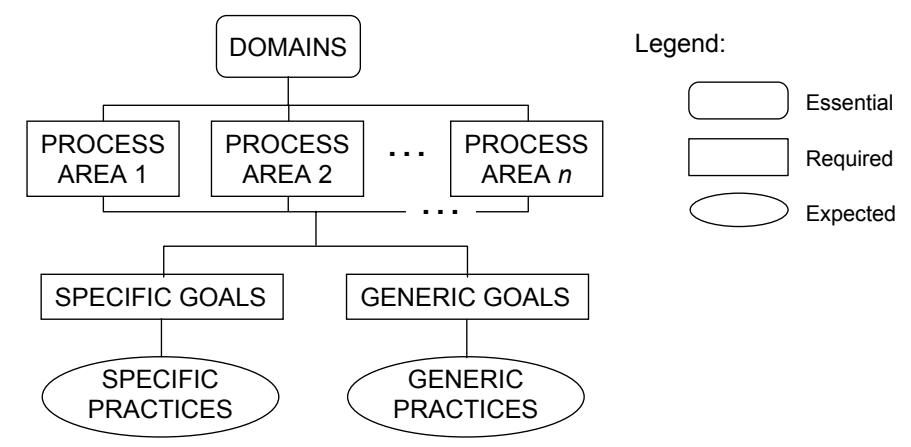

Figure 4.2: Structure of the ICoNOs MM.

in architecture (as presented in the previous paragraphs), it is expected that our model incorporates six levels of maturity - as the CMMI does in its continuous representation [57]. However, we want to offer a roadmap to approach B-ITa improvement (i.e., series of maturity levels) focusing on a set of B-ITa process areas. Such process areas must provide CNOs with specific practices characterized by each maturity level. Therefore, we decided to include five levels of maturity (see Figure 4.3) in the ICoNOs MM (as in the staged representation of the CMMI). That is, when using the ICoNOs MM, process improvement results are summarized in a single maturity level number for each of the B-ITa domains that the model includes.

In our model, levels are used to describe an improvement path recommended for a CNO to perform processes in order to achieve B-ITa. To reach a particular level, a CNO must satisfy all the set of process areas that are targeted for improvement in a particular B-ITa domain. The levels are:

Level 1: Incomplete. At maturity level 1, processes related to a particular B-ITa domain are usually not performed or partially performed. It means such a particular domain is not explicitly considered when a CNO strives for B-ITa. Therefore, this level contains no processes in our model.

Level 2: Isolated. At maturity level 2, processes are the basic infrastructure in place to support a particular B-ITa domain. They (i) are planned and executed in accordance with a policy; (ii) employ skilled people who have adequate resources to produce controlled outputs; (iii) are monitored, controlled, and reviewed. However, such processes are isolated initiatives that are not managed from the entire CNO perspective.

Level 3: Standardized. At maturity level 3, processes are directed to make improvements in the standardization and management of a particular B-ITa domain. Processes are performed from a CNO perspective (i.e., they are cooperative initiatives). They are well characterized and understood, and are described in standards, procedures, tools, and methods. 


\subsection{COMPARATIVE ANALYSIS OF THE MODELS}

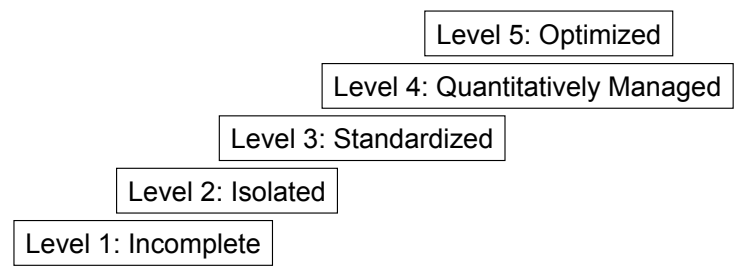

Figure 4.3: The B-ITa levels in the ICoNOs MM.

Level 4: Quantitatively Managed. At maturity level 4, processes use statistical and other quantitative techniques. Quantitative objectives for quality and process performance are established and used as criteria in managing the process. Quality and process performance is understood in statistical terms and is managed throughout the life of the process.

Level 5: Optimized. At maturity level 5, processes are improved based on an understanding of the common causes of variations inherent in the process. The focus of an optimized process is on continuously optimizing the range of process performance through both incremental and innovative improvements.

\subsubsection{Domains in the model}

Not all models presented in Section 4.1 identify B-ITa domains, and thus they limit the identification of improvement opportunities on particular B-ITa-related areas. The ICoNOs MM includes four domains, i.e. partnering structure, IS architecture, process architecture and coordination. Chapters 5 and 6 describes the focus group and the case studies conducted to identify and validate such B-ITa domains.

\subsubsection{Process areas in the model}

Similarly as in the 'domains' criterion, the 'process areas' criterion shows that not all analyzed models include process areas in them. Only the MAAM and COBIT present a list of specific good practices that need to be performed in order to improve alignment. Chapter 7 discusses the theoretical and empirical studies that we conducted to identify the processes included in the ICoNOs MM.

\subsubsection{Alignment level and orientation of the model}

Only two of the models (the CIO Council's assessment guide and the MAAM) are explicitly focused on assessing operational issues related to B-ITa. The CIO Council's assessment guide provides tools to assess technical alignment, and the MAAM covers aspects that focus on existing organization structures and operational processes in an organization. The rest of the models seems to make no 


\section{CHAPTER 4. RELATED WORK}

clear, explicit commitment on the level of alignment to where they direct to. Our ICoNOs MM is focused on operational B-ITa. Operational B-ITa involves organizational issues as communication, structure and, particularly, coordination processes that operationalize the business aspect of organizations in specific actions to perform [335]. It consists of aligning the operational activities of IT and people to each other so that optimal IT support for business requirements is achieved. This aspect is reflected in the process areas included in the ICoNOs MM. Our model is the first MM that explicitly assesses B-ITa in a CNO context. In the ICoNOs MM, higher maturity will be a guarantee for smooth operations running in a CNO for improving B-ITa, but cannot be a guarantee that the business model of the network will be $100 \%$ profitable, or that the $\mathrm{CNO}$ will have a best-of-class competitive position.

In the previous two sections, we have presented and compared different approaches that are useful to assess B-ITa. We have explained how the knowledge involved in the design of these approaches is useful for the design of the ICoNOs MM. For example, similarly to BITAMA and COBIT, our model is a development MM; and similarly to the MAAM, our model includes not only domains but also specific process areas. In the next section, we present MMs for CNOs to be aware of how we can include a collaborative perspective in the ICoNOs MM.

\subsection{Maturity models for CNOs}

Since we are developing an MM for CNOs, our literature review also covers some MMs that can be applied in collaborative settings. These models do not assess B-ITa. They only cover particular domains related to B-ITa in CNOs (e.g. architecture or processes) disregarding other necessary domains that need to be taken in consideration by CNOs when achieving B-ITa. In the following, we briefly present six of these models. Our purpose is to identify what characterizes these models that makes them to be MMs for CNOs settings. Only after that we can define what is important to include in our model to cover a collaborative perspective.

\section{The EIMM}

The Advanced Technologies for interoperability of Heterogeneous Enterprise Networks and their Applications (ATHENA - http://www.athena-ip.org) is a project that aims to contribute to the interoperability issue by producing results that span the full spectrum of interoperability from technology components to applications and services, from research \& development to demonstration and testing, and from training to evaluation of technologies [166]. In the context of ATHENA, interoperability is not limited to ISs, but interoperability also relates to business processes and the business context of the organizations. ATHENA claims that the effect of interoperability avoids large budgets being spent on time-consuming 


\subsection{MATURITY MODELS FOR CNOS}

system integration tasks and helps to achieve seamless business across organizational boundaries in a $\mathrm{CNO}$ environment.

One of the methods developed in the ATHENA project is the Enterprise Interoperability Maturity Model (EIMM). It is an MM that "defines a set of area of concerns and a set of maturity levels that provide the means to determine the current ability of an enterprise to collaborate with external entities and to specify the path to improve this ability" [18, p. 7]. The EIMM covers six areas of concern (business strategy and processes, organization and competences, products and services, systems and technology, legal environment including security and trust, and enterprise modeling) and five maturity levels: (1) performed, (2) modeled, (3) integrated, (4) interoperable, and (5) optimizing.

The ATHENA team claims that the EIMM is an staged model based on the structure of the CMM. Therefore, similarly to the CMMI and our ICoNOs MM, the EIMM is a process-oriented MM. However, this model does not only deals with processes. It also deals with deliverables that have to be in place in order to reach each of the maturity levels. It seems that the EIMM only helps to set to what extent functional areas of collaborating organizations are ready to interoperate.

\section{The IT outsourcing MM}

Adelakun [2] developed an MM to assess the collaboration between organizations involved in outsourcing relationships specific for IT. Particularly, the model helps to find the level of IT functions outsourcing. The author claims that organizations engaged in outsourcing relationships are at various level of maturity. Disregarding the motivation for outsourcing, an organization can be classified in one of the following five levels of maturity:

1. Insourcing. In this level organizations buy a few IT functions or services from external vendors on short term contractual agreements only.

2. Experimenting. In this level organizations collaborate in outsourcing of IT activities. These outsourcing relationships have no pattern or order in time.

3. Storming. In this level organizations need to make a decision either to pursue outsourcing actively or to keep on experimenting.

4. Norming. In this level organizations pursue IT outsourcing actively and establish norms, methods or processes for IT outsourcing.

5. Performing. In this level the IT outsourcing is part of the strategy and it is not only based on cost reduction or improvement but on strategic matters.

The model is a useful tool for organizations that would like to follow a stepwise maturity stage in their IT outsourcing relationships. However, it is focused on the IT side of organizations only, thus leaving the collaborative business side out of its scope. 


\section{CHAPTER 4. RELATED WORK}

\section{The extended CMM}

The extended CMM (as its name indicates it) is an extension of the original SEI SW-CMM. Ramasubbu et al. [250] claim that the CMM lacks process areas to manage distributing software projects, such as establishing mutual knowledge and managing geographically dispersed social networks. The extended CMM covers 24 new process areas that address the processes required for managing software engineering in a collaborative environment using virtual teams [239]. Those process areas build upon four domains: collaboration readiness (defined as the ability to set goals that can easily being translated into maintainable tasks in the virtual team), common ground (defined as the shared knowledge between the software engineers who reside in different locations), coupling in work (defined as the division of work and roles in the virtual team), and technology readiness (defined as the IT and personal capability levels for using collaborative technologies); and are organized in three maturity levels: initiation, consolidation, and high productivity.

Because the motivation for developing this model was to extend a singleorganization-oriented MM to address a collaborative perspective, this model explicitly covers aspects related to collaboration. However, the focus of the model lies on software engineering only.

\section{The E2AMM}

The Extended Enterprise Architecture MM (E2AMM) [280] is a model to assess the enterprise architecture of collaborating organizations. This MM lies on the premise that development of the enterprise architecture is vital for collaboration "because it provides the rules and definition necessary for the integration of information and services at the operational level across organizational boundaries" [280, p. 1]. The E2AMM covers aspects related to involvement of the organizations, collaborative budgets, strategic governance, and the holistic view of IT between the participants in the CNO. So, the model addresses business and IT aspects in a holistic way covering one organization and its environment. Because of this, Schekkerman [280] believes that the E2AMM helps to guarantee a natural alignment between business and IT. However, B-ITa is in this model addressed at the strategic level and as a domain in the model, only. The model does not identify process areas required to improve such an alignment.

\section{The SCM MM}

Lockamy and McCormack [185] have developed a Supply Chain Management (SCM) MM based on business process orientation issues and empirical evidence from a variety of industries. The model presents five levels of maturity: ad hoc, defined, linked, integrated and extended. The model illustrates the way to go from individual planning of supply chain processes to central planning of processes. It presents the increased integration of the core management processes (plan, source, make and deliver) when moving to more mature levels in the model. The 


\subsection{MATURITY MODELS FOR CNOS}

highest level of this model (i.e., the 'extended' level) refers to CNOs comprising a collaborative culture in their supply chain management issues. According to Lockamy and McCormack [185] trust and mutual dependency is essential for a CNO to function properly.

This model can successfully help organizations that collaborate in a supply chain. However, from our context (i.e. B-ITa MMs for CNOs), that is exactly its shortcoming: it is limited to assess SCM issues.

\section{The CollabMM}

CNOs have been relying upon collaboration for productivity improvement in order to achieve cost reduction or to gain revenue. However, CNOs cannot assure collaboration is properly conducted in every-day operations. In response to this, the CollabMM [193] has been developed. CollabMM is a collaboration MM for business processes, "proposed in the belief that organizations can benefit from explicitly taking collaboration into account when thinking about, modeling and defining their business processes" [194, p. 305]. The model describes an evolutionary path in which processes can achieve progressively higher maturity on collaboration through four maturity levels: ad hoc, planned, aware, and reflexive. Each specific level comprises a group of related activities, which can be executed together, for the purpose of improving the process collaborative capability. The model only serves to assess aspects as coordination, communication, and knowledge sharing between the participating organizations in a CNO.

\subsubsection{Discussion on the observations}

All six models outlined in the previous paragraphs present explicitly a collaborative perspective. This collaborative perspective is mainly focused on business processes and integration level (as the EIMM, the SCM MM, the E2AMM, and the CollabMM do), relationships (as the IT outsourcing MM does), and information and IT sharing (as the extended CMM does). All these issues cover aspects of the collaborative perspective. We decided to include this perspective also in the IT and processes practices included in the IS architecture and process architecture domains of our model (see Chapter 8).

However, we believe that an MM that ascribes to a collaborative view also has to include coordination mechanisms, structure and organizing principles (partnering structure aspects). As it will be seen, from our case study research, we have strong indications that, even if the architectures of ISs and processes are properly taken into account in collaborative B-ITa projects, coordination and partnering structure must also be considered by CNOs assuring more positive results in such projects (see Chapters 5, 6 and 7). 


\subsection{Summary}

In this chapter, we presented a review of pertinent literature on B-ITa MMs and MMs for CNOs. B-ITa MMs have been presented in order to identify improvement goals and reusable knowledge to address and use in the development of the ICoNOs MM, respectively. Furthermore, we also introduced several MMs for CNOs in order to define what is important to include in our model to cover a collaborative perspective. In the following, we summarize these reusable knowledge and the improvement goals.

Reusable knowledge: our ICoNOs MM will...

- be a development MM (similarly to BITAMA and COBIT).

- include not only domains but also specific process areas (similarly to MAAM and CMMI).

- have five maturity levels (similarly to CMMI in its staged representation).

- assess operational B-ITa (similarly to the CIO Council's assessment guide and MAAM).

- include the collaborative perspective in IT and process aspects (similarly to the MMs for CNOs we presented).

Improvement goals: our ICoNOs MM will...

- be a continuous MM (similarly to one of the representations of CMMI).

- assess B-ITa in CNOs and not in single organizations.

- include the collaborative perspective in organizational coordination and structure aspects. 


\section{Part II}

\section{Solution}





\section{5 B-ITa domains: The initial attempts}

\subsection{Introduction}

In Section 4.2.1 of Chapter 4, we have introduced the development process we followed to build the ICoNOs MM. Based on the structure of the CMMI, we illustrated the components of our MM (see Figure 4.2): domains, process areas, goals and practices. Domains are sets of process areas that share common knowledge of aspects within the scope of the MM (in our case, a domain is a group of processes which need to be performed by CNOs in their efforts for improving B-ITa - we call them 'B-ITa domains'). Process areas are sets of activities that are performed to make improvements in a particular domain. Goals describe characteristics that must be present to satisfy process areas, and practices describe what may be implemented to achieve the goals. Part II of this dissertation presents all these components of the ICoNOs MM. In Chapters 5 and 6 , we deal with the domains of the model. The process areas, the goals and the practices are introduced in Chapters 7 and 8 , respectively.

This chapter in particular describes how we used a focus group session and a case study to identify the first set of B-ITa domains included in our ICoNOs MM. Our goal was to investigate two aspects of the ICoNOs MM, namely the suitability and the completeness of the first set of domains included in it. Our first selection of domains was based on literature review and interviews with experts. The validation effort reported in this chapter represents the first step out of all the steps we followed to find the domains that are ultimately included in our MM (see Chapter 6).

The reminder of this chapter is organized as follows: in Section 5.2, we briefly present (i) the first proposal of the B-ITa domains included in the ICoNOs MM and (ii) validation aspects of an MM. Section 5.3 describes our validation process. Section 5.4 and Section 5.5 respectively discuss how we used the focus group session and the case study to evaluate the B-ITa domains. Section 5.6 discusses the results of the validation approach. Finally, Section 5.7 summarizes the chapter. We make the explicit note that in this chapter we present the first set of domains included in the ICoNOs MM. The final B-ITa domains are presented in Chapter 6 after using three other case studies for generalization of findings. 


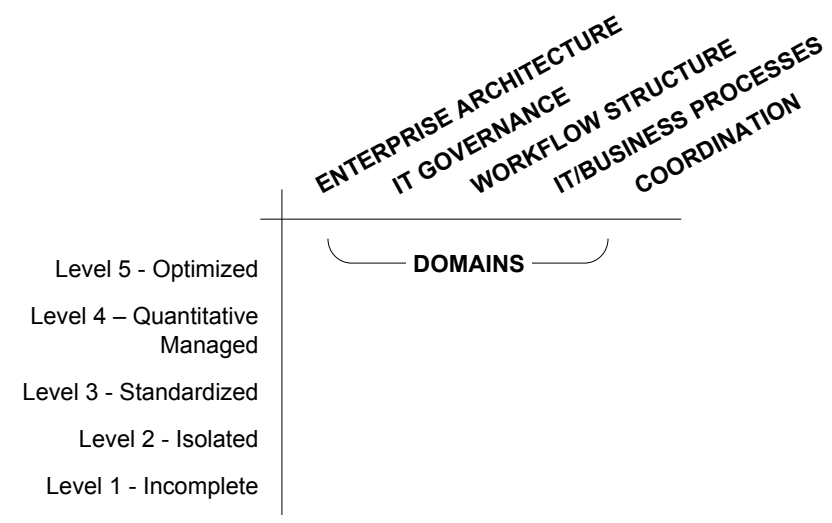

Figure 5.1: First proposal of the B-ITa domains.

A preliminary version of this chapter has been published in the proceedings of the 11th IEEE International EDOC Enterprise Computing Conference [273].

\subsection{B-ITa domains and their validation}

Based on our reviews of B-ITa MMs and MMs for CNOs (see Chapter 4), and on literature related to business/IT processes and CNOs - e.g., see Chapter 2 and articles as $[123,167,214,207,266]$, we chose the domains to be considered by collaborations in their efforts for achieving B-ITa. These domains were those B-ITa concepts that we identified important in our reviews, and they were: enterprise architecture (IS landscape), IT/business processes, workflow structure, IT governance, and coordination (see Figure 5.1). We present the definition of each of these domains in turn.

- Enterprise architecture, defined as the landscape of ISs, the relations between them, the technology infrastructure on which they run, and the way they create value for the organization (based on Zarvic's work [351]).

- IT/business processes, defined as the architecture of all processes needed to reach the shared goals of the collaborating organizations. These processes are both primary business processes of the CNO and processes needed for information exchange among the collaborating organizations.

- Workflow structure, defined as the specification of the roles and responsibilities with respect to the IT and business processes that comprise the previous domain.

- IT governance, defined as the "leadership, organizational structures and processes that ensure that the enterprise's IT sustains and extends the organization's strategies and objectives" $[144$, p. 5]. 


\subsection{THE VALIDATION PROCESS}

- Coordination, defined as the mechanisms to manage the interaction and work among the participating organizations taking into account the dependencies and the shared resources among the IT/business processes.

Before using an MM for day-to-day operations, it needs to be validated. The validation of MMs received significant attention since the early days of the CMM [235]. In particular, the initial validation efforts related to CMM focused on comparing the costs and quality of the software engineering process of organizations that used the CMM with those who did not. For example, Schlumberger [347] and Motorola [86] used CMM-based improvement programs as a vehicle for implementing productive changes and to evaluate if the CMM provided real benefits. This kind of validation can be conducted only when a model has been completed (see EVALUATE step of the MM development process presented in Section 4.2.1 of Chapter 4). Apart from these attempts [86, 347], with very few exceptions [13], existing literature offers almost no advice on how to empirically validate an MM. Therefore, validation is still a big challenge for MMs researchers.

Based on recommendations by researchers in empirical software engineering evaluation [164] and requirements engineering [75, 334], one way to approach the validation of MMs is to provide evidence that the model is in fact useful. This implies to investigate it by empirical means in order to understand it, to evaluate it, and to deploy it in proper contexts. This helps to assure the design of a useful model [133]. The validation of the ICoNOs MM as a whole will be presented in Part III of this dissertation. In this chapter, we only present the B-ITa domains that we identified after conducting a focus group and a first case study to validate them.

According to Beecham et al. [13], a valid MM is a model that is sufficiently accurate to achieve its purpose. The purpose of our MM is to enable assessing B-ITa in CNOs to plan future B-ITa actions while evolving toward a culture of process improvement excellence. With this purpose in mind, the ICoNOs MM is considered valid if it allows CNOs to assess their alignment. Therefore, for the purpose of this chapter, validity comprises the completeness and suitability of the domains included in our model to reach this goal. The domains are the enablers of B-ITa in CNO settings that will be assessed using the model. The B-ITa domains will be complete when we include all necessary suitable domains that can enable such alignment. For identifying all those domains we conducted the focus group and the case study presented in this chapter and three other case studies (see Chapter 6) which helped us (i) to cumulate ideas in order to generalize findings concerning the necessary suitable B-ITa domains and (ii) to support their inclusion in the ICoNOs MM.

\subsection{The validation process}

As shown in Figure 5.2, our validation strategy proposed to carry out a focus group session followed by a case study. The rational for this combination order 


\section{CHAPTER 5. B-ITA DOMAINS: THE INITIAL ATTEMPTS}

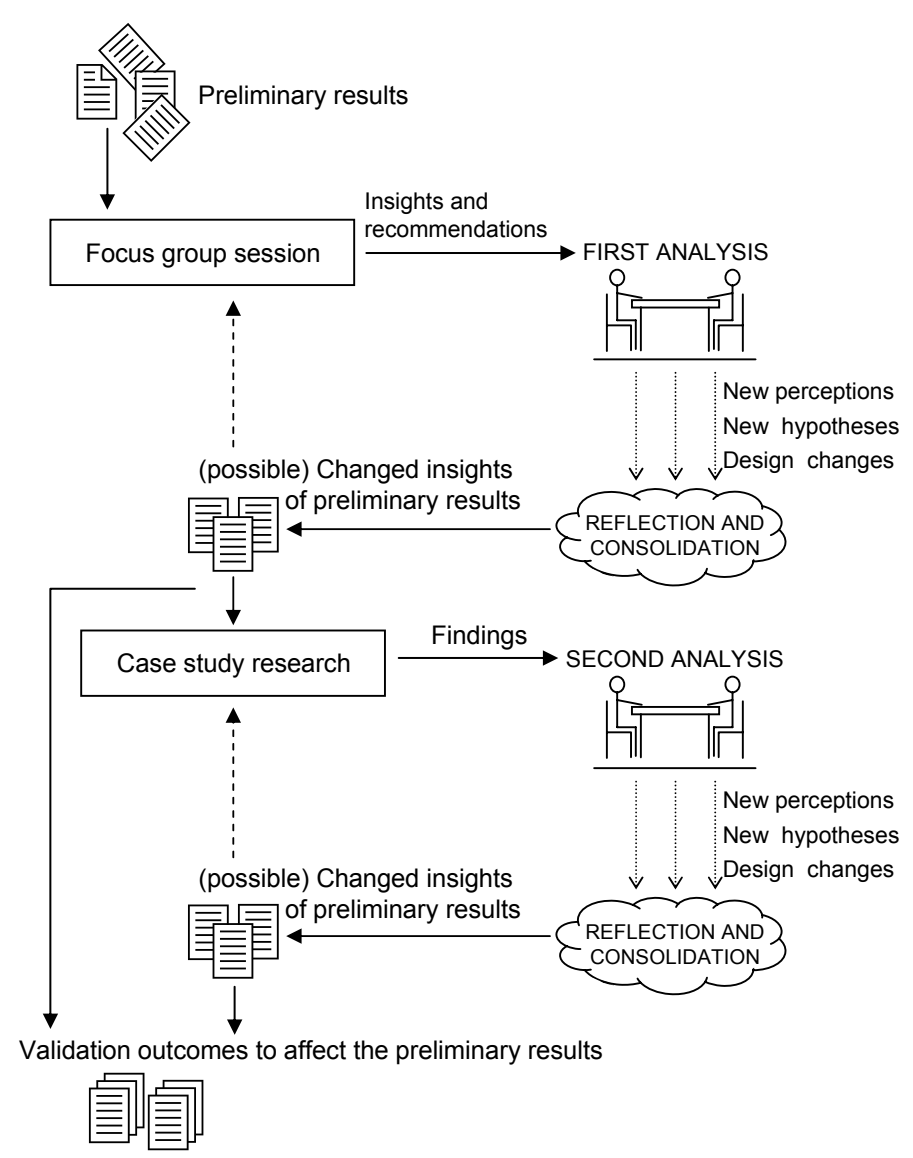

Figure 5.2: B-ITa domains validation approach.

of research methods was to get initial data that would give new insights into the B-ITa domains before beginning to identify these domains in a real-life CNO. Our approach is consistent with recommendations by methodologists [66, 289] who point to the use of a focus group session to gain insights into the preliminary results of the design of a model. The collected insights must be used to develop new perspectives of such a model and to derive new hypotheses or changes in the model's design. A case study is furthermore conducted to clarify the design options worth considering when enhancing the model's definition. The main activities involved in our validation process were:

\section{FOCUS GROUP}

1. Formulate and communicate the purpose of the group session.

2. Bring together professionals to participate in the focus group.

3. Conduct the focus group presenting the domains included in the model. 


\subsection{THE VALIDATION PROCESS}

4. Let professionals speak, share ideas and discuss.

5. Stimulate professionals to move their thoughts from general reactions to specific recommendations.

6. Treat disagreements among professionals by inviting them to re-discuss topics.

7. Register the spoken recommendations.

8. Analyze how recommendations could derive new hypotheses or changes on the design of our model.

9. Report results of the focus group session.

\section{CASE STUDY}

10. Formulate and communicate the purpose of the case study.

11. Present the project to our contact partners in the CNO.

12. Receive and classify documentation about the case study from the contact partners.

13. Read the documentation to identify information concerning the domains.

14. Analyze and report the findings of the case study.

15. Compare case study's findings to the results of the focus group session.

16. Adapt the ICoNOs MM so that it reflects the results of the validation.

While running the validation process, we kept in mind that the most important task was to constantly watch for the distinction between relevant information and trivial data. We considered relevant those information pieces which told us something of interest about the connection between the B-ITa domains and the knowledge we possessed concerning them. As we wanted to track in the documentation received in step 12 only those information pieces which related to each of the domains included in the ICoNOs MM, we focused on those documents that presented information related to (i) IT, (ii) processes and (iii) governance. In addition, to identify the workflow structure, we referred to contract documentation and the SLAs. Although no coordination mechanisms were explicitly mentioned in the documents, we could identify them (see Section 5.5.2).

When looking for information pieces related to the B-ITa domains, we analyzed the data contained in the documents to detect visible information patterns or trends in the documents. This process involved searching for both "wholes" as well as "gaps" in the data. In addition, when analyzing data in documents, we thought about the consistencies and inconsistencies of information patterns that we were discovering.

The next two sections discuss in detail the two validation instruments we used in our validation strategy. How the insights, which we gained from the focus group session and the case study, impacted our model is discussed in Section 5.6. 


\subsection{Focus group session}

In order to generate a deeper understanding of the first set of domains presented in Section 5.2 and also to validate that they indeed were relevant domains to ensure B-ITa in CNOs, we started conducting a focus group [171, 212, 353]. We involved professionals, who research aspects related to the B-ITa domains, in an active discussion. The goal of the focus group session was to understand how suitable and complete the first five B-ITa domains were.

Our focus group session included 20 professionals and was facilitated by the author of this dissertation who presented the domains and the rational for their consideration in collaborative B-ITa efforts. While we targeted professionals from different background as recommended by Kitchenham et al. [163], it turned out that the professionals' availability was the key factor determining their attendance to the focus group session. The professionals were drawn from the Information Systems Group of the University of Twente (http://is.cs.utwente.nl) and the business partners participating in our research project. Table 5.1 classifies the focus group participants based on their expertise.

Table 5.1: Distribution of participants' expertise in the focus group session.

\begin{tabular}{l|c|c|c|c|c}
\hline Field of expertise & A & B & C & D & Total \\
\hline \hline Business-IT alignment & 1 & 1 & 1 & & 3 \\
Software systems & & 1 & 2 & 1 & 4 \\
Security & & 1 & 1 & & 2 \\
Workflows & & 1 & 1 & & 2 \\
Cooperative work & & & 1 & & 1 \\
Requirements engineering & & & 2 & & 2 \\
Enterprise architecture & 2 & & & 3 & 5 \\
Coordination processes & 1 & & & & 1 \\
& & & & & \\
Total & 4 & 4 & 8 & 4 & 20 \\
\hline
\end{tabular}

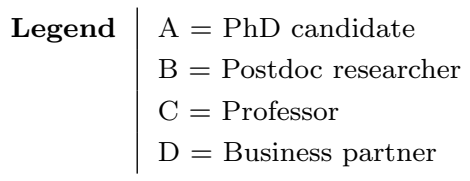

The focus group was conducted in a manner consistent with the steps covered by moderated structured focus group sessions [124]. That is, we first explained that the goal of the focus group was to verify the suitability and completeness of the first five B-ITa domains included in our ICoNOs MM. Then, we presented an overview of the MM. Specifically, we concentrated in the B-ITa domains. After that, the discussion was started by preplanned primary questions as: Are the B-ITa domains realistic from researcher and practitioner perspectives? Are the B-ITa domains understable? What are the potential problems in using or 


\subsection{FOCUS GROUP SESSION}

understanding the B-ITa domains? As the focus group progressed, follow up question where also posed. For example, after asking the question What are the potential problems in using or understanding the B-ITa domains?, a follow up question used in the focus group was: Are there any omissions or gaps in the B-ITa domains? We make the explicit note that in our focus group session several questions and comments were directed among focus group participants. That is, instead of only posing questions to the participants and then listening to the answers, the facilitator made comments to participants about what other participants have stated and then asked for a response. This encouraged the discussion among participants.

The facilitator of the session motivated the participants to share ideas and led the discussion assuring that the focus group was not dominated by a small number of participants. In addition to the facilitator leading the discussion, in the focus group one other researcher who was involved in the development of the ICoNOs MM was also presented. He was making notes during the session and participating in the discussion on the basis of his experience and knowledge. His comments were not later analyzed, while his insights were considerably helpful when we treated the insights of the other focus group participants, and when discussing results with him and with the other researcher involved in the development of the ICoNOs MM.

\subsubsection{Results}

We identified six key points that came out as a result of the focus group session:

1. 9 professionals found that in general the term 'enterprise architecture' covers all the other presented B-ITa domains.

2. Having in mind the definition of enterprise architecture that we presented, professionals thought IS architecture or IS landscape was a better name for this domain.

3. 57 percent of the professionals raised the question: "why to include workflow structure as a separated domain if IT governance includes already some definition of roles and responsibilities?"

4. There was a strong consensus amongst the professionals that the name of the domain workflow structure must change. It is to avoid its confusion with the most common perception of the term workflow, i.e., automation of a business process, in whole or part, during which information is passed from one participant to another based on some rules [3].

5. Professionals indicated that - in their experiences - coordination concepts and IT/business processes could not exist in separation. They found that some coordination mechanisms are always used to glue cross-organizational process fragments together and make them run smoothly. 
6. A final concern that three professionals had was: why not to consider the collaboration as a single organization and then apply models for assessing B-ITa in single organizations (see Chapter 4) for assessing?

Because the ICoNOs MM is a new MM to assess B-ITa, the participants' opinions on other B-ITa MMs (see Chapter 4) and their knowledge of the CMMI could affect their perception of our model. The final concern that three professionals had, i.e., "why not to consider the CNOs as single organizations and then apply current B-ITa MMs for assessing B-ITa?", in our opinion stems from a difference in the way of thinking that professionals adopt. There are two kinds of professionals' thinking: bottom-up and top-down thinking [26, 83]. The bottom-up thinkers explain phenomena by first looking at higher level self-organized patterns of low level entities. They solve a particular problem by first trying out the available theories, methods and tools deeming appropriate. The top-down professionals act the other way around: they would start observing phenomena and, from a particular behavior, they would develop theories, methods and tools to solve the problem. In our research, we apply the top-down way of thinking, as we conjecture that B-ITa is fundamentally different in a CNO context. For example, the lack of a central decision maker necessitates CNOs be approached from the perspective of economic theories [272] that simply do not apply in the single organization case. Adopting a bottom-up approach, then, poses the danger of overlooking this important issue.

The discussion of the rest of the results is presented in more detail in Section 5.6 after conducting the case study. This let us to use the results of both research methods when defining their implications for the ICoNOs MM.

\subsubsection{Focus group validity threats}

The major limitation of our focus group session was that it was a single focus group, which restricted the extent to which generalizations could be drawn from its outcomes. As Morgan states [213], generalizations are likely appropriate only to professionals in settings similar to the setting of our focus group members. In this respect, we considered the data as 'incompletely collected' [212, 213], meaning that what we collected was the experience of the professionals only. Furthermore, we acknowledge that a plan of at least 3 or 4 focus groups, as methodologists suggest [171, 213], would have brought much richer results. However, we could not follow such a recommendation because of resource constraints at the time we had the first set of B-ITa domains to include in the ICoNOs MM.

Although Morgan [213] indicates that there is no hard evidence that the focus groups moderator's impact on the data is any greater than researcher's impact in participant observation or interviewing, a validity concern in focus groups is that the researcher influences the group interaction. We mitigated this threat in our focus group by objective participation, i.e., by providing an appropriate amount of guidance to the participants without involving or introducing any of our own opinions in the discussions. This assured the internal validity of our focus group 


\subsection{CASE STUDY 1: TECHNOLOGY OUTSOURCING RELATION}

session (see Chapter 3). We also were conscious that the participants themselves could influence the data they produced. For example, by means of imbalanced level of participation in the discussions. We made sure that the focus group was not dominated by a small number of very active participants and that everyone got a chance to express their ideas.

\subsection{Case study 1: Technology outsourcing relation}

In this section we describe an exploratory study which examined the collaborative work between two organizations. Its objective was to investigate whether and to what extent the B-ITa domains were present in the CNO investigated. Specifically, we wanted to identify important information concerning (i) each of the domains and (ii) new important topics to consider in CNO B-ITa attempts.

\subsubsection{Research site}

The first CNO we studied was an outsourcing relationship between a leading international business and technology integrator and a local provider of mass-marketed services (hereinafter referred to as: Insourcer and Outsourcer, respectively).

Insourcer is an international information technology services company, providing consultancy, systems integration and managed operations. Outsourcer offers a wide range of services for both the private and business market. It focuses on the Benelux-countries and Germany. The stock of both Insourcer and Outsourcer is traded on Euronext, the pan-European stock exchange, where both companies are in the top 100 in terms of market capitalization.

Outsourcer decided to outsource part of its IT operations to Insourcer in 2001. This was a measure to confront the company's troubled IS architecture management. The 2001 architecture consisted of home-grown applications, stove pipe solutions, and a lot of point-to-point connections. The company experienced problems related to inconsistent data, significant operational expenses, and below-average customer satisfaction. The outsourcing measure had the objective to help (i) provide continuity of service to the customers so that number of complaints was reduced, (ii) improve financial results due to purchase price and cost reduction, and (iii) optimize the IS architecture and performance.

The relationship between the two organizations was based on a joint-go-tomarket approach in the regional market. This means that Insourcer provided consulting, systems integration and managed operations services to Outsourcer; however, at the same time Insourcer received services from Outsourcer. It was thought to be a win-win outsourcing relationship. Insourcer is the major supplier for Outsourcer and it delivers almost all of their portfolio assets. The most important assets are datacenter and application management, desktop services, and software house activities. On the other hand, Outsourcer delivers a wide range of services to Insourcer. 


\subsubsection{Data sources and findings}

After our research project was presented to our contacts in Outsourcer, they provided documentation concerning their collaborative work with Insourcer. We found three types of documents useful for the purpose of our case study and we carefully reviewed them. These were:

- Letters and memoranda concerning a variety of B-ITa topics in their collaborative work.

- Agendas, minutes and reports of meetings that partners of the two organizations attended to discuss alignment issues and solutions.

- Partner relation management documents, e.g., contracts and SLAs.

All documents were created in the period 2004-2005, and they were the only ones available to us. According to Yin [348], these types of documents are useful even though they are not always accurate and, though, they can report bias, i.e., they can reflect the bias of the professionals who wrote them. Documents must be carefully used and should not be accepted as literally recording of information and events. The documentation we obtained was mostly clear. However, there were some unexplained acronyms and figures. This brought confusion into our interpretations. In total, we received fifteen documents where we could find how the outsourcing partnership arose, what was wrong and what were the agreements on solving the B-ITa problems. We present our findings as follows:

Enterprise architecture. The available documents presented just a big picture of the application landscape of Outsourcer to show the organization's IT reality prior to the collaboration with Insourcer. Outsourcer had a home-grown application landscape with stove pipe solutions and a lot of point-to-point connections. The company had inconsistent data, big expenses and could not manage the client service in an appropriated way what caused poor customer satisfaction. Dependencies among the partner's ISs in the entire CNO were not found.

IT/business processes. The documents showed nothing concerning the processes that each participating organization performed when they started to collaborate, or concerning the collaborative processes. One document reported that a result of setting up the outsourcing relation, was the allocation of all tasks (that together constitute the joint processes) to the business partners in a clear and consistent way. However, these processes themselves were not documented. At least not in the set of documents available to us. The definition of processes that could be found in the documentation was related to the Outsourcer's processes that were not functioning properly. This was to have a common understanding on how jointly, Outsourcer and Insourcer could improve such processes.

Workflow structure. The industry sector in which Outsourcer operates is almost constantly changing in a fast pace. Issues related to the workflow structure domain of Outsourcer were therefore very complex and constantly changing. 


\subsection{CASE STUDY 1: TECHNOLOGY OUTSOURCING RELATION}

Outsourcer addressesed this complexity in a 'divide-and-conqueror' way by distinguishing three main IT branches:

1. IT systems that are part of the national service distribution network maintained by Outsourcer,

2. IT products and services offered to their customers, and

3. the IT systems that support the business processes of Outsourcer.

This last IT branch was the first one to be outsourced. In due course, Outsourcer's datacenter, end-user support and software house were also outsourced, all to Insourcer. Outsourcer and Insourcer together developed a general governance model and guiding principles for their cross-organizational collaboration to monitor and control the agreements on rights and responsibilities.

IT governance. What went wrong in this $\mathrm{CNO}$ were the decision making mechanisms (e.g., there was lack of ownership and assigned responsibilities) and the inconsistent level of business commitment (e.g., there was lack of clarity and no compliance to agreements). Therefore, in the process of setting up the collaboration, Outsourcer and Insourcer defined leadership roles, distributed responsibilities, and identified the activities needed to improve the deployment of the IT potential to support Outsourcer's business objectives, e.g., an IT management manual was developed and strategic/tactical IT platforms were installed.

Coordination. In this collaboration, we observed reciprocal dependencies [304] between the two participating organizations. Such a situation led to establish a considerable number of coordination mechanisms to control the collaborative work. We identified at least seven coordination mechanisms used in support of the outsourcing partnership: the goals identification, the SLAs specification, the KPIs definition, the schedules and guiding principles establishment, the service framework agreement, the expectations definition and communication, and the payments right specification. All these mechanisms were directed to achieve concerted actions and common goals between the two organizations.

These findings suggested that the coordination and the definition of roles and responsibilities (i.e., workflow structure in our ICoNOs MM) were the most important topics for the work between Outsourcer and Insourcer. It implied a level of agreement on mutual expectations, thus, it reduced ambiguity in the collaboration for a better operation. The considerable number of documents presenting issues related to ISs and IT, led us to conclude that Outsourcer and Insourcer spend more time in the specification of enterprise architecture issues than of IT/business processes. However, we make the explicit note that such IS/IT issues reported in the documents were not presented at CNO level. That is, documents only reported the IS As-Is status of Outsourcer as single organization.

Beside enterprise architecture, IT/business processes, workflow structure, IT governance, and coordination, cost management also was a domain that strongly 
affected their B-ITa efforts. Their B-ITa key drivers were to control costs and to manage risk. Once Outsourcer and Insourcer were engaged in their outsourcing relationship, fixed expenses were (partially) established. Regardless of market stability, the costs were predetermined and thus Outsourcer could make plans over a period of time through a budget. This was a key to the financial projections of Outsorcer in floundering finances to strive against the below-average customer satisfaction it experienced. Outsourcer reduced cost by outsourcing not only new IT projects, but also ongoing IT functions. Its job after outsourcing was to manage risks and preserve those parts of the organization that were the essential core competency for safeguarding a good future.

\subsubsection{Case study validity threats}

The documentation was read and analyzed by two of the researchers who were involved in the development of the ICoNOs MM. Having done such analysis by ourselves may reflect some bias in how we interpreted the data. The researchers worked in isolation from each other in two locations and met only after all documents were read. We consistently observed a consensus in our findings. Whenever there was disagreement, the points of disagreements were discussed until both researchers arrived at a consensus. We then believe that the threat to validity due to researchers' bias was minimal, because no one of the researchers was in a close research collaboration or work relation with the studied CNO or with the professionals who delivered the reviewed documents. Also, we continuously evaluated our findings (by reflexivity and self-checking) because the subjective nature they could have.

Furthermore, as this was a single case study, clearly we could not generalize results. More data needed to be collected over other CNOs to replicate or counter the results of this case study . Only then, convincing evidence could be established and results could be considered robust and worthy to generalize. We considered this as our most important issue and, therefore, it topped our agenda for immediate future research. We planned to conduct more case studies to replicate findings by a series of follow-up studies that progressively helped us to refine our understanding about the B-ITa domains (see Chapter 6). Finally, the report of the case study results was reviewed by a third researcher (peer debriefing strategy) who also was involved in the development of the ICoNOs MM.

\subsection{Implications of the results for the B-ITa domains}

The recommendations obtained from the focus group session and the findings of the case study were used to make decisions on what to change in the domains so that their completeness and suitability were improved. Our decisions concerned both the choice of names for the B-ITa domains and their definitions, and the inclusion of a new domain. Figure 5.3 shows (the relationships among) the new B-ITa domains. These new domains are partnering structure, IS architecture, 


\subsection{IMPLICATIONS OF THE RESULTS FOR THE B-ITA DOMAINS}

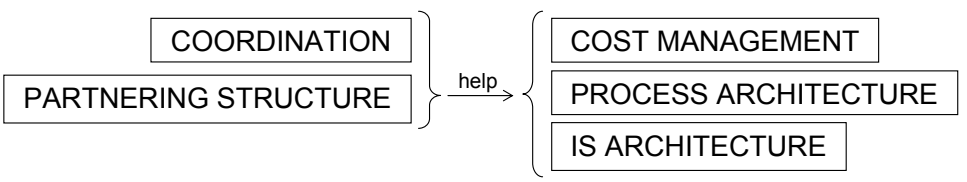

Figure 5.3: The 'new' domains of the model.

process architecture, coordination, and cost management. The analysis of the results of the focus group session supported us to take the following immediate actions:

- we renamed the domain 'enterprise architecture' to 'IS architecture' to better reflect its new scope. This scope is apparent in the new definition, which we present below.

- we renamed the domain 'IT/business processes' to 'process architecture' to involve both the collaborative IT and business processes without discriminating between these two types.

- we renamed the domain 'workflow structure' to 'partnering structure' to emphasize its contents of how cross-organizational work gets done and who is involved including the definition of roles and responsibilities, and organizational structures.

- we merged the domain 'IT governance' with the domain 'partnering structure', which meant that the definition of the domain partnering structure incorporates all aspects of what was earlier termed IT governance.

- we considered 'cost management' as a new B-ITa domain.

Below, we present the definitions of the resulting five B-ITa domains:

- Partnering structure, defined as the cross-organizational work division, organizational structure, and roles and responsibilities definition that indicate where and how the work gets done and who is involved. It helps to give structure not only to the participants involved in activities of the IT side or the business side of a CNO, but also creates the required connection for business and IT to move forward together. Partnering structure helps to organize the collaborative work.

- IS architecture, defined as the fundamental organization of the information management function of the participating organizations embodied in the ISs, i.e., software applications, that realize this function, their relationships to each other and to the environment, and the principles guiding its design and evolution (based on IEEE 1471 [195]). It must be noted that, in our work, we distinguish IS architecture from IT architecture. For us, IT architecture consists of the software and physical infrastructures we defined in 
Section 2.1.3 of Chapter 2. That is, (i) the implementation platform, i.e., the collection of standard general-purpose software needed to run the IS architecture. It ranges from operating systems (OSs), middleware, network software to database management software; and the (ii) physical network, i.e., the physical resources that run the software applications. This includes computers, cables, wireless access points, printers, and user interface devices to support the running of the IS architecture [316]. We present an explicit distinction of these two architectures in the next case study presented in Chapter 6. Hereinafter, we refer to the IT architecture as IT infrastructure architecture to make its context clearer.

- Process architecture, defined as the choreography of all processes needed to reach the shared goals of the participating organizations. These processes are both primary business processes of the $\mathrm{CNO}$ and processes needed for information exchange.

- Coordination, defined as the mechanisms to manage the interaction and work among the participating organizations taking into account the dependencies and the shared resources among the processes.

- Cost management, defined as the discipline of ensuring IT is obtained in the most effective price - which does not necessary mean cheapest - so that the CNO can understand the cost of its services and/or products (based on ITIL [148]).

Talking about B-ITa commonly leads one to think in aspects related to technology (IS architecture), processes (process architecture), (strategic) goals - see Chapter 2, and even cost management (as the case study presented in this chapter proves). However, this is a misunderstanding when talking about B-ITa in CNOs. In collaborative B-ITa projects, beside those 'easily perceived' aspects, coordination and partnering structure are vital for assuring the success of the projects. These two B-ITa domains are general domains in the sense that they are not directly related to B-ITa but they help to create an environment where B-ITa improvements can be easily achieved. If coordination and partnering structure are not considered in collaborative B-ITa projects, B-ITa efforts could turn more complex than they already are. Participating organizations in CNOs strive for the provision of complex products or services that they cannot offer in their own as single organizations. We have found that in a CNO, coordination does not imply managing the relations between the customer-face organization and the rest of the participating organizations only. Coordination also implies to manage (i) the relations among all the participating organizations and (ii) the work required to produce the products/services. In theory, this is moving from a tree structure to a network topology which implies to find a balance between market and hierarchical coordination.

Beside coordination, partnering structure must also be considered when striving for collaborative B-ITa. CNOs need (i) to use authoritarian relationships (re- 
lations plus roles and responsibilities definition) among the participating organizations, and (ii) to effectively apply such an authority to manage the work commitment. It can be argued that CNOs and hierarchy/authority relationships are two terms that cannot be seen as siblings. However, we believe the opposite. A case in point is that of IBM and its Microelectronics Joint Development Alliance consortium (including Siemens, Samsung, Freescale, Infineon, and STMicroelectronics) to develop semiconductor technologies [236]. As consortia often do it, this consortium conceded to one participating organization (IBM) the right to organize the consortium and to determine the behaviors of the other participants within a given context to coordinate their actions, to speak on everybody's behalf and to exert leadership, i.e., to use authority. The complete organizational structure, the course of actions for achieving results, and the norms and policies on the organizational roles are also important partnering structure aspects to consider in collaborative B-ITa.

We claim that partnering structure, IS architecture, process architecture, coordination and cost management are the domains to be considered when dealing with CNOs so that value is created for the participating organizations and B-ITa is improved. We make the explicit note that using a multi-method approach could not be enough to overcome external validity concerns that both a case study and a focus group are exposed to. For example, in our case study, (i) the assumptions we made for our MM are not representative for all CNO B-ITa projects that exist, and (ii) the investigated B-ITa project in the economic sector might not be representative even for all the B-ITa projects in this economic sector, or in the public sector. We then decided to conduct three more case studies to cumulate knowledge and replicate findings to confirm the B-ITa domains in new real-life settings (see Chapter 6).

\subsection{Summary}

In this chapter, we started to investigate the validity of the ICoNOs MM. We used a focus group session and a case study for this purpose. This validation was not the evaluation of the entire model itself. Instead, its sole purpose was to validate the B-ITa domains included in the MM. We merged focus group and case study methods to form a validation approach that we found helpful in assessing maturity models in their initial stage of development. In our validation process, we experienced the advantages of the complementary use of the two methods. Coupling findings from the focus group session and from a case study provided a much richer context for identifying appropriate refinement actions on our model.

The results gained through the two reseach methods used in this study let us increase our knowledge of the completeness and the suitability of the B-ITa domains. Results of the focus group session suggested that some domains were not properly termed or defined. The name of these domains caused some confusion among the professionals. Therefore, we decided to rename or merge some of the previously domains of the first proposed set. We conducted the focus group 
session prior to the case study so that initial data were used to have new insights into the domains before their identification in the case study. The objective of the case study was, then, to track in documentation those pieces of information relevant to each of the domains, so that we could see what concerns were important for the particular CNO we studied. The case study findings suggest that IS architecture, coordination and partnering structure are the most important topics to consider when dealing with CNO settings. However, after analyzing the results of the reseach methods, we believe that partnering structure, IS architecture, process architecture, coordination and cost management are the set of B-ITa domains that should be considered by CNOs. 


\section{6 B-ITa domains: Empirical validation}

\subsection{Introduction}

This chapter presents the continuation of the steps we followed to identify the domains that are ultimately included in our MM and to which the maturity levels of our MM apply. We are elaborating on the last step of the design of the model (see the MM development process we present in Chapter 4). Based on our findings, we propose to consider four key domains for successful B-ITa in CNOs: partnering structure, IS architecture, process architecture and coordination. Our claim is that improvements in these domains cause more improvements of B-ITa in collaborations across organizations than improvements in other domains do. These domains are the most frequent way of working found for B-ITa improvements in three real-life CNOs.

B-ITa domains within single organizations have been studied by other researchers in their efforts to build B-ITa MMs (see Chapter 4). Those studies show that domains such as skills, technology scope, partnership, governance, competency measurements, communications, informal organization, requirements and IT architecture help to align business and IT in single organizations. However, our claim is independent of the results of such studies since we focus our research on collaborations across independent organizations as opposed to on single organizations. We empirically validated our domains by means of three case studies conducted in (i) a cross-organizational collaboration among governmental departments of the state of Tamaulipas in Mexico, (ii) a networked organization between the province of Overijssel, the municipalities of Zwolle and Enschede, the water board district Regge \& Dinkel and Royal Grolsch N.V., all in the Netherlands; and (iii) a network of government agencies in Canada.

The remainder of this chapter is organized as follows: in Section 6.2 we describe the multiple case study research approach we followed. Then, we present the three case studies conducted to validate the B-ITa domains discussing their individual findings in Sections 6.3, 6.4, and 6.5, respectively. Section 6.6 presents a cross-case analysis. Finally, we summarize the chapter in Section 6.7. 


\subsection{Multiple case study research approach}

We conducted a multiple case study to evaluate the validity of the following claim:

In any successful B-ITa improvement process in a CNO, improvements in partnering structure, IS architecture, process architecture, coordination and cost management cause more improvements of $B$ ITa than improvements in other domains do.

This claim was derived from the results of the focus group and the first case study we presented in Chapter 5 . Using a multiple case study research approach, we investigated whether the domains mentioned in such a claim are indeed the BITa domains to take into account by CNOs, or whether it is required to consider more domains in CNO B-ITa efforts. Specifically, we wanted to identify both important information concerning each of the five domains and new valuable topics characteristic to B-ITa attempts in CNOs that could be considered as candidates for forming new B-ITa domains. This helped us to produce detailed answers to the questions of why and how B-ITa improves, for whom, and in what circumstances (see the description of our realist approach in Chapter 3).

A high-level view of our overall research setup is presented in Figure 6.1. It shows the way we conducted the multiple case study research in three CNOs. As described in Chapter 3, a multiple case study differs from a set of single case studies in that a multiple case study helps to investigate a phenomenon within its real-life context to answer the same research question in different single case study sites. We followed a replication logic [348] when conducting these case studies. That is, after conducting the first study, we uncovered significant findings (empirical statements) that led us to conduct two more studies with replication as our immediate research goal. According to Klein and Myers [165], Pawson and Tilley [230] and Yin [348], only with replication of findings, such findings could be robust for generalization.

In Chapter 3, we have already stated that the sites we studied were not randomly sampled. We used purposive sampling to select the case study sites. Our main criterion for selecting the studied CNOs was the collaborative network perspective which the CNOs take in their efforts towards improving B-ITa. Once this criterion was met, the only other two requirements were that the CNOs explicitly had a B-ITa project and that they were willing to grant us access to it. We did not intend to conduct a comparative study across CNOs, but rather to enrich our understanding by bringing different insights from each CNO. In this sense, it is important to consider the particular context of each CNO when interpreting the data. Therefore, we chose a hermeneutic approach to collect data. However, we followed the realistic approach of Pawson and Tilley [230] to analyze the data (see Chapter 3 for more explanation). In our particular case, a hermeneutic approach helped us to obtain results from analyzing the information sources, the sites, and their organizational contexts altogether. In addition, the realist approach helped us identifying the possible underlying mechanisms for 


\subsection{MULTIPLE CASE STUDY RESEARCH APPROACH}

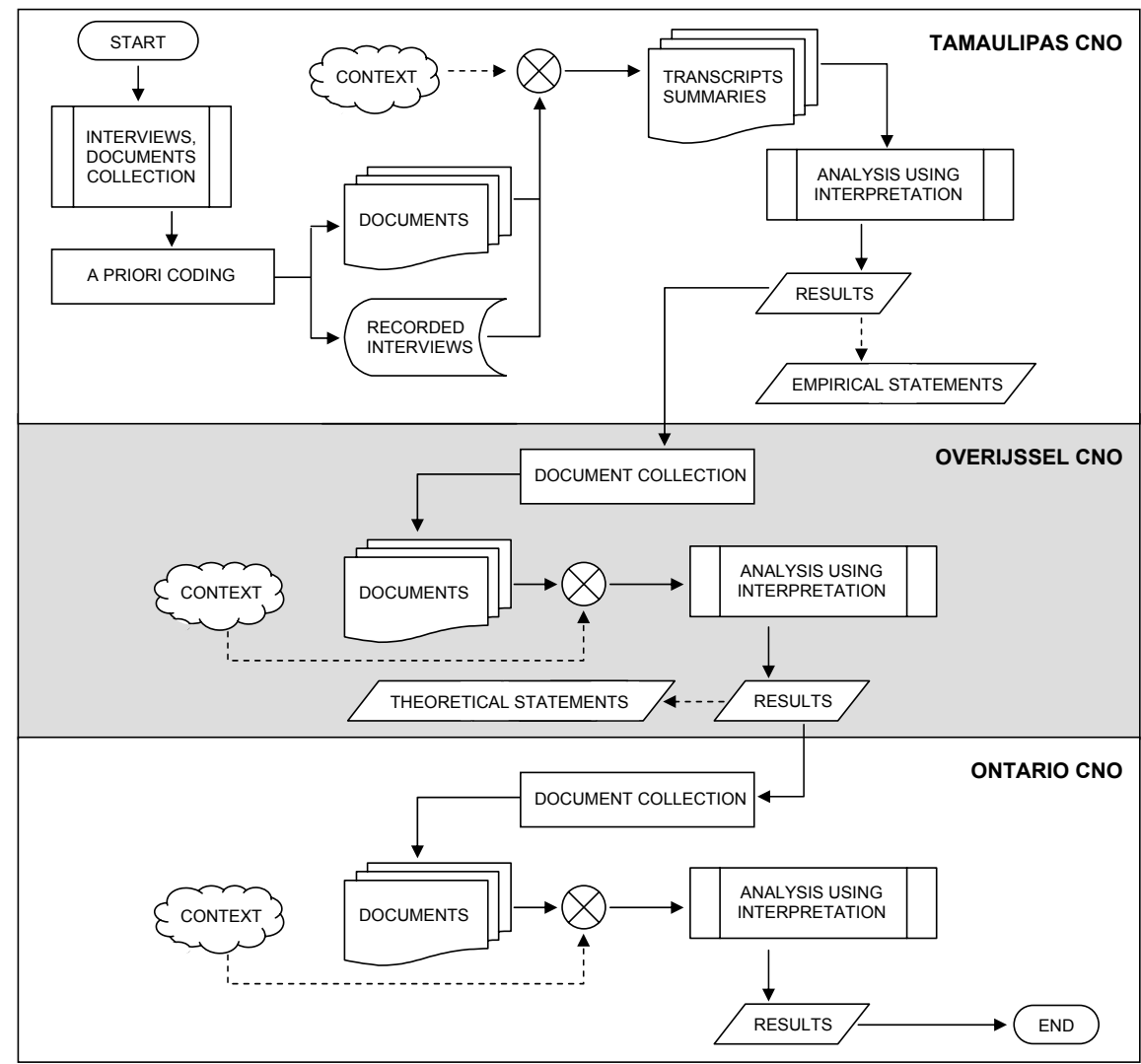

Figure 6.1: Multiple case study research approach.

what we observed and to relate them to the B-ITa domains in the context of the particular cases. Because we were interested not only in the mechanisms but in the minimum number of B-ITa domains that must be considered by CNOs for making B-ITa improvements, we added solution (S) to the context-mechanismoutcome configuration (CMO configuration) of Pawson and Tilley [230] - see Chapter 3, and called this new situation as a "CSMO configuration'. The S in our context is what helps to improve B-ITa (i.e., the B-ITa domains). We oriented our thinking to CSMO configurations. This led to the development of transferable and cumulative ideas to state what it is about a CNO B-ITa project which works for whom in what circumstances.

To properly come up with a refined CSMO configuration, we used the research cycle for theory building presented in Figure 6.2. The claim presented in the beginning of this chapter represents the theory that, according to Pawson and

\footnotetext{
${ }^{1}$ This configuration type has been previously discussed by Denyer et al. [84] using the term 'CIMO configuration', where I stands for 'intervention'. An intervention is considered as an action (or a set of actions) to be taken in solving a specific problem.
} 


\section{CHAPTER 6. B-ITA DOMAINS: EMPIRICAL VALIDATION}

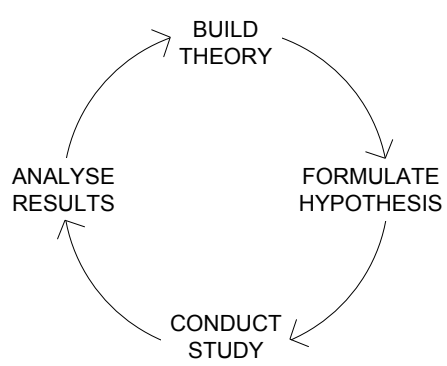

Figure 6.2: A research cycle for theory building (adapted from $[230,150]$ ).

Tilley [230], needs to be translated into a clear hypothesis. Such a hypothesis must explain what might work for whom in what circumstances. At the point of our work when we had only conducted one case study in a technology outsourcing relation (see Chapter 5), our hypothesis was:

Partnering structure, IS architecture, process architecture, coordination and cost management are B-ITa domains that must be considered by CNOs in their efforts for improving B-ITa.

We started with the CSMO configuration presented in Figure 6.3. That is, in a technology outsourcing relation between two profit-and-loss responsible organizations, the B-ITa domains (solution from a CSMO configuration viewpoint) must be implemented in order to improve B-ITa. This initial CSMO configuration presents no mechanisms. A mechanism must be seen as a way in which the B-ITa domains are permeated into the reasoning of the team that work for improving B-ITa (i.e., a manner to apply the B-ITa domains). We make the note that throughout this chapter we explicitly indicate - when necessary - in square brackets [ ] what is a context, (a part of) a solution or a mechanism.

\begin{tabular}{|c|c|c|c|c|c|c|}
\hline Context & + & Solution & + & Mechanism & $=$ & Outcome \\
\hline \multirow{5}{*}{$\begin{array}{l}\text { Technology outsourcing } \\
\text { relation between two } \\
\text { organizations in the private } \\
\text { sector }\end{array}$} & & Partnering structure & \multirow{5}{*}{+} & & \multirow{5}{*}{$=$} & \multirow{5}{*}{$\begin{array}{l}\text { B-ITa } \\
\text { (improvements) }\end{array}$} \\
\hline & & IS architecture & & & & \\
\hline & & Process architecture & & & & \\
\hline & & Coordination & & & & \\
\hline & & Cost management & & & & \\
\hline
\end{tabular}

Figure 6.3: Initial CSMO configuration.

As per the realist approach, each of the case studies in this chapter represents then one iteration of the research cycle (see Figure 6.2) along the way to evaluate the plausibility of our hypothesis which, in turn, helped us to support the inclusion of the B-ITa domains in our ICoNOs MM. The case studies were steps 


\subsection{CASE STUDY 2: STATE GOVERNMENT COLLABORATION}

forward to assure that our ICoNOs MM makes sense to business practitioners who work in real-life CNOs.

The data in the case studies were collected during January 2005 and October 2008. The data collection technique used in each CNO was individually selected to match the particular settings. This choice was motivated by the resources at our disposal. When planning the case studies originally, we considered the use of interviews. However, at case study execution time, it turned out that we could collect data through interviews in one case study site only. In the other sites, we used documents as data sources. Throughout the three case studies presented in this chapter, we made refinements of the initial CSMO configuration (see Figure 6.3) based on the individual findings in each case study. Specifically, the discussion sections after reporting on the findings of each case, present the new solution and mechanisms we were finding. In each refinement, we cross out part of the solution, mechanisms and/or context explanations to show they are not anymore considered in the CSMO configuration. The case studies are presented in the next sections.

\subsection{Case study 2: State government collaboration}

The second CNO we studied in our research was a network of more than a hundred departments of the state of Tamaulipas in Mexico (hereinafter referred to as Tamaulipas CNO). The United Mexican States (Mexico) is a federal constitutional republic, i.e., a federation of thirty-one free and sovereign states and a Federal District. Tamaulipas is one of these self-governing states. Since the beginning of the administration 1999-2004, the organization of the Tamaulipas state government has not changed considerably. The government is divided in 12 secretaries. In average, each of these secretaries has 4 divisions and each division consist of at least two departments. One of these divisions is the Division of ICT (Information and Communication Technology) that is responsible for all ICT activities in the government of Tamaulipas, including development of new systems (IS architecture) and maintaining the IT infrastructure that support these systems. This division is the supplier of IT services within the government of the state of Tamaulipas. It is the IT side in the B-ITa problem. The business side (IT demand) is represented by the requirements of all other secretaries, divisions and their departments (see Figure 6.4). The demand side drives the identification and prioritization of systems requirements and opportunities to exploit emerging technologies. This separation of IT management issues (i.e., supply and demand sides) in the collaboration among the secretaries of the Tamaulipas state government is a situation that fits properly with the operational B-ITa we are addressing.

The government of the state of Tamaulipas shares the view that modern governments must be distinguished by the results that they bring, by the solutions that they generate, and by the opportunities and transparency that they offer to the society. As a response to the necessity of having a modern government admin- 
CHAPTER 6. B-ITA DOMAINS: EMPIRICAL VALIDATION

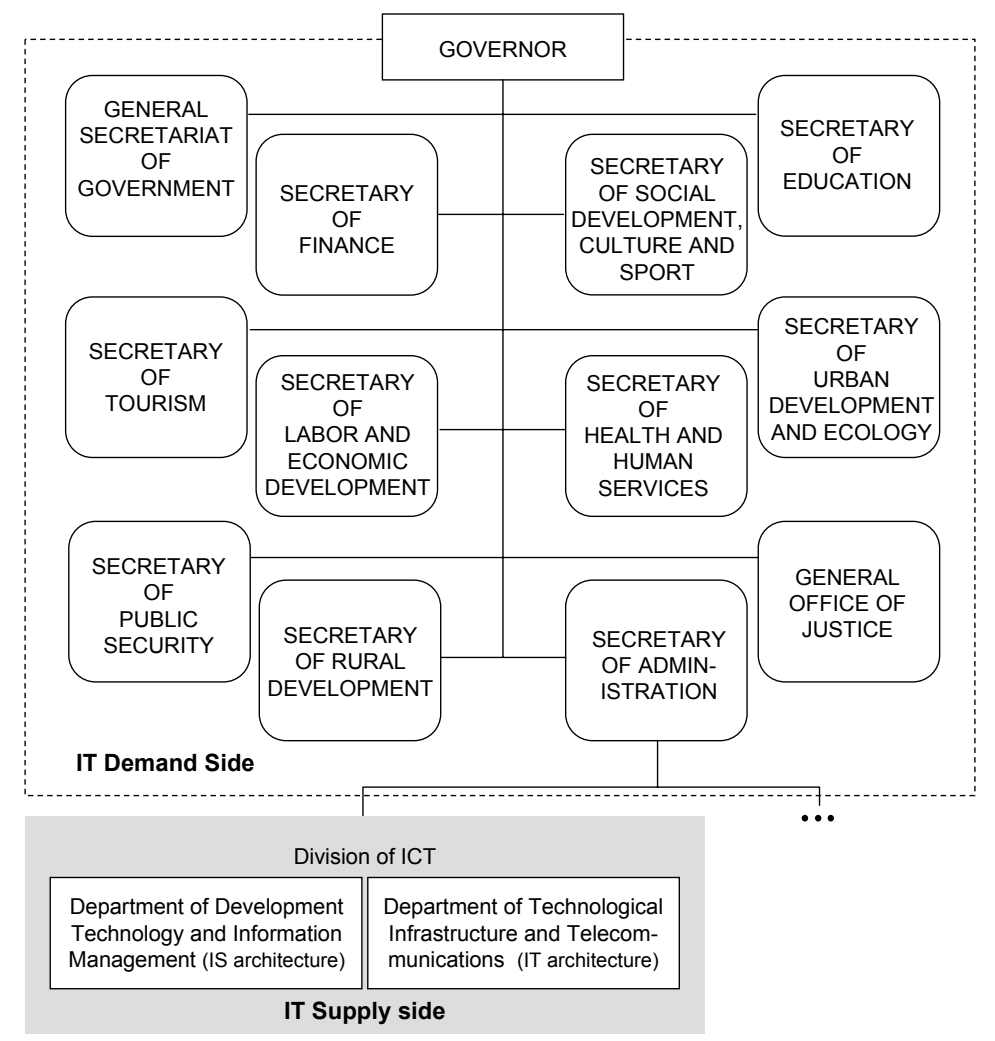

Figure 6.4: IT and business sides in the Tamaulipas government structure.

istration, the government of the state of Tamaulipas implemented Domino/Notes to allow the departments to maintain fast and uninterrupted internal communication, while offering better quality of service to citizens. The goal of the project was to increase e-mail uniformity and to allow the development of collaborative systems. This goal faces the overall requirements of the government departments: to make the service-delivery process more effective and efficient, and to create a better government-citizen relation, meeting the expectations of society.

The first project under Domino/Notes was the Citizen Attention Service System (CASS). This system helps to collect all the individual requests and petitions that the citizens raise to the governor and to the government secretaries chiefs, i.e., any request concerning public services as electricity, security and the like, that the citizen wants to submit to the government of the state of Tamaulipas. The CASS project began in 2001. The initial situation in the area of service provisioning to citizens was characterized by much bureaucracy and poor response time. Only few of the departments had a system to manage the requests. Those systems were home-grown applications developed by IT sections of different departments. Each had its own application logic and data semantics and contributed in a unique way 


\subsection{CASE STUDY 2: STATE GOVERNMENT COLLABORATION}

to a lack of homogeneity and communication among systems. For example, when a department received a citizen's request that involved other department(s), the official documents were sent by internal postal service and the department in charge had no longer direct control on the request. This control came back when the documents were received back. The communication among departments was primarily by means of telephone.

The new system facilitates the allocation, distribution and communication of citizens' requests among departments, as well as all the information related to such requests, i.e., the associated preceding events, the elaboration of the official document, the current status and the answer of the departments involve in the process to satisfy the requests. This helps to have better control in each of the processes, while having a close relation with the citizens to keep them informed on the process of their requests. A visible advantage after implementing the CASS was the reduction of response time. The system records the citizens' requests and automatically sends them to the departments that are involved, thus avoiding bureaucracy and driving employees to work efficiently because of the feeling of being controlled by superiors by means of the new system.

\subsubsection{Research design and findings}

The upper layer in Figure 6.1 illustrates the analysis process we followed for this case study. In the Tamaulipas CNO we used semi-structured interviews with an average duration of 1 hour per interview. The five B-ITa domains covered in the hypothesis to test were thought of in terms of five general questions in the form of: "was <DOMAIN> considered in the Tamaulipas CNO for the CASS project?" (where <DOMAIN $>$ was replaced with each of the five B-ITa domains, i.e., partnering structure, IS architecture, process architecture, coordination and cost management). In addition, we asked a sixth question in order to identify whether the Tamaulipas CNO had considered different domains into account for its B-ITa improvements. To get in-depth explanations of the domains, follow-up questions were asked at interview execution time. All interviews were taped to help writing the transcripts which we used for analyzing. The interviews were conducted with 6 persons on a one-to-one basis. It can be argued that interview data is often biased by impression management [116] and retrospective sensemaking [331]. However, we mitigated such a bias by interviewing different and highly knowledgeable professionals who view the Tamaulipas CNO from diverse perspectives since their expertise were different.

According to literature [98, 348], an "a priori codification" of expected concepts is valuable when starting an analysis process because it helps to shape the theory to be tested and built. Our previous work [273] helped us to develop such codification based on the domains included in our ICoNOs MM, i.e., partnering structure, IS architecture, process architecture, coordination and cost management. This codification was later used in the analysis of the interviews transcripts. If the codes proved important in the results of our case, then we had a firmer empirical ground for the findings. 


\section{CHAPTER 6. B-ITA DOMAINS: EMPIRICAL VALIDATION}

Individual transcripts summaries were created from each participant's recorded interview. This process helped to develop a clearer picture of the answers of the participants to the questions of the interview. Specifically, the transcriptions were useful to carry out a 'within-case' analysis [98], i.e., the interviews write-ups helped to generate insights and to cope with secondary data of the case study site. As the interview data was, in the end, summarized in transcripts, using hermeneutics we iterate between reflecting on the whole text and reflecting on its parts (e.g., set of terms). In our particular case, having as basis the help of stakeholders and domain experts (i.e. the focus group session), a hermeneutic approach helps to obtain results from analyzing the information sources, the interviewees, and the organization altogether in a specific context to establish case boundaries and use that understanding in turn to improve our understanding of the different parts of our data $[227,230]$. In addition, analysis of secondary data was done in parallel to the analysis of the interview transcripts. The secondary data was collected from the documentation which referred to aspects of the domains included in our model. Proceeding from the case knowledge and case study results, we made some inferences about the B-ITa domains. The validation of these inferences depended on the plausibility of the logical reasoning that is used to describe the results and to build theory [165]. Therefore, the results of the Tamaulipas CNO study served as empirical statements that were input for the next case study (see Section 6.4).

For this case study, we based our analysis process on theories, frameworks, and principles developed by case study research methodologists [165, 175, 348] who claim that the generalization from theoretical statements (e.g. a theory confirmed in one setting) to empirical statements (descriptions of other settings) is possible and valid. So, to strengthen the claim that the theory we built from previous efforts (i.e., the claim derived from the research presented in Chapter 5 and presented in the beginning of this chapter) is indeed generalizable to new settings, we are conducting this study to confirm the theory in a new but similar setting.

\section{Findings}

Partnering structure. The state of Tamaulipas, as a public organization, has a hierarchy of authority with powers and responsibilities understood by all, a clear-cut division of work among the departments and people, and an explicit set of procedures for making decisions. The government of the state of Tamaulipas, every four years, upon the start of a new administration, checks and revises its 'Regulations, Procedures and Organization Handbook'. The complete organizational structure, the description of roles and responsibilities, the course of actions for achieving results, the norms and policies on the organizational roles, and the relationships among them, can all be found in this handbook. As one of the interviewees pointed, it means that, for the CASS project, "the departments already had (i) a good definition of roles and responsibilities, and (ii) an established governance structure". Although they have assigned responsibilities to the individual actors collaborating in the network, what went wrong was the inconsistent 


\subsection{CASE STUDY 2: STATE GOVERNMENT COLLABORATION}

level of commitment to work effectively with a minimum waste of time and effort. In the collaboration, the points of power and authority were positioned in places which rendered it ineffective to mitigate such a situation. It was no effective use of authority to manage the work commitment program.

IS architecture. Most of the interviewees expressed that "the documentation of the IS architecture of the complete cross-organizational collaboration was indeed necessary when the CASS project began". However, it turned out that this architecture was not enough to reach the goals of the project. The IS architecture included a significant number of home-grown applications and this rendered inefficient any approach to integrating them and setting up a solid foundation for CASS. This situation led to start the development of CASS from scratch. It must be noted that for the government of the state of Tamaulipas the documentation of the architecture at the level of software applications was not enough. They also needed to define the architecture of infrastructure and technical issues (the IT infrastructure architecture). For the CASS roll-out team to know which departments were ready for CASS and which were not, it was important to have an inventory of the hardware and OSs of each of the participating departments. This inventory effort revealed that they needed to acquire new hardware to support the new collaborative system. "Several departments had very obsolete hardware and OSs that differed from the OSs that most of the departments had".

Process architecture. After deciding to implement Domino/Notes as new platform to develop systems in the Tamaulipas state government, the definition of process architecture became an important task for the success of each project that followed. For the government of the state of Tamaulipas, this architecture was necessary to align the new systems, e.g., CASS, with the requirements of the cross-organizational collaboration. Unlike our previous case study (see Chapter 5), where the studied CNO gave more priority to the IS architecture domain than to the process architecture domain, the government of the state of Tamaulipas begins a project by thinking first about the processes and, then, about the ISs. First, they define the processes that each participant will perform, as well as the collaborative processes. Then, they analyze what ISs could support such processes. In their case, these supporting systems were new systems as they lacked effective applications.

Coordination. In this $\mathrm{CNO}$, and particularly in the CASS project, the requests and petitions management process depends on several departments (for example, Table 6.1 presents the departments involved in the educative credit request process - this credit is given to students who want to pursue their university studies in other Mexican states). Such a situation led to establish a considerable number of coordination mechanisms to help control the collaborative workflow. Our interviewees converged on the use of the following mechanisms: (i) coordination enabled through shared goals, (ii) coordination enabled through agreements specifications, (iii) definition and communication of mutual expectations, and 


\section{CHAPTER 6. B-ITA DOMAINS: EMPIRICAL VALIDATION}

Table 6.1: Educative credit request process.

\begin{tabular}{|c|l|l|c|}
\hline STEP & ACTIVITY & DEPARTMENT & DAYS \\
\hline 1 & Submit educative credit request & Student & 1 \\
\hline 2 & Receive the request and check the academic situation & Division of Finance and Administration & 1 \\
\hline 3 & Pursuit the request process & Department of Service of the ICEET & 5 \\
\hline 4 & Verify the data of the credited and endorsement & Department of Investments and Portfolio & 8 \\
\hline 5 & Approve/reject the credit & Subcommittee of the Trust of the ICEET & 2 \\
\hline 6 & Elaborate the contract and the promissory note & Institute of Educative Credit & 2 \\
\hline 7 & Get signatures of the credited and endorsement & Division of Finance and Administration & 1 \\
\hline
\end{tabular}

ICEET = Instituto de Crédito Educativo del Estado de Tamaulipas

\begin{tabular}{l|l} 
Total steps & 7 \\
Total days & 20 \\
Total departments & 5
\end{tabular}

(iv) regular control meetings. All these mechanisms were used in combination to achieve concerted actions among the participating departments, i.e., to coordinate the mutual work treaties in the state of Tamaulipas.

\subsubsection{Discussion}

The findings of this study suggested that partnering structure, IS architecture, process architecture and coordination are indeed domains that CNOs take into account in their B-ITa efforts. This replicates part of the results of the case study presented in Chapter 5. However, the government of the state of Tamaulipas also considered the IT infrastructure architecture as domain to address when striving for B-ITa. The Tamaulipas CNO case helped us indeed to strengthen our claim. Improvements in partnering structure, IS architecture, process architecture and coordination [solution] indeed cause more improvements of B-ITa in a CNO context. For example, in the Tamaulipas CNO, although persons who had roles involving power and authority could not assure that others committed to work effectively, the documentation of the IS architecture and the IT infrastructure architecture, and the definition of the collaborative processes created a situation where the CNO properly could make the ISs supported its requirements (which were implicit in the defined processes). Coordination helped them to collaborate in a way that the CNO could make real B-ITa improvements.

We have found no more evidence to support the importance of cost management for B-ITa improvements. However, we found that IT infrastructure architecture [solution] is also a domain to consider for assuring B-ITa improvements in the public sector [context]. Unlike the findings of the first case study we present in Chapter 5, the government of the state of Tamaulipas begins a project by 


\subsection{CASE STUDY 2: STATE GOVERNMENT COLLABORATION}

thinking first about the processes and, then, about the ISs. This led us to think that the order in which the domains are taken into account [mechanism] in the efforts to achieve B-ITa does not affect the efforts' results. The importance of the domains varies according to the settings where a CNO functions. In the efforts for aligning ISs with the requirements of the business, each CNO can begin its B-ITa effort first in the domain that best meets its objectives.

The Tamaulipas case study also helped us to identify that the level of automation (through the existing systems) of the participants in a $\mathrm{CNO}$ affects considerably the degree of collaborative B-ITa. A previous study [152] on cultural perceptions into single organizations IT management found that, although managers were aware of the requirements to achieve B-ITa, B-ITa was practically not feasible because the organizations perceived the importance of IT as very low in the first place and therefore there was very limited attention for it. The results of the Tamaulipas case study suggest that this situation - where B-ITa is not achieved as a result of low IT importance - can be extrapolated to CNOs where single-owned ISs are essential for the collaborative work. If the social status of IT is low and the participating organizations do not have any automated ISs that could be used to support the collaborative processes [mechanism], efforts for achieving CNO B-ITa can take much more time in comparison to B-ITa efforts in collaborations with considerable level of automation of its activities. Our observation was that if automated systems do not exist, there are no collaborative B-ITa improvements. The first refinement of our CSMO configuration is presented in Figure 6.5.

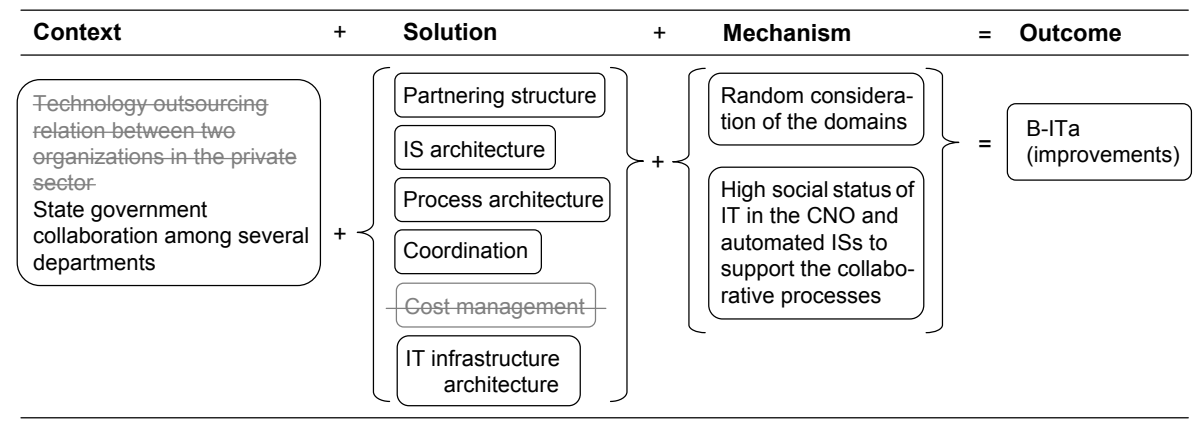

Figure 6.5: First refinement of the CSMO configuration.

From this refinement, the resulting B-ITa domains are partnering structure, IS architecture, process architecture, coordination and IT infrastructure architecture. These domains help indeed to improve B-ITa in a CNO. The cost management domain was not replicated in our results. Therefore, it falls out the CSMO configuration after this first refinement. Following recommendations by Lee and Baskerville [175] to generalize findings, with these results we could derive empirical statements from the theoretical statements created in the previous case study. However, because our interest lays on cumulating ideas to have strong evidence for the inclusion of the B-ITa domains in the ICoNOs MM, we decided to conduct 
two more case studies (which we present in the next two sections).

\subsection{Case study 3: Regional government network}

Our third case study was conducted in a Dutch networked organization that included agencies at the level of provincial and municipal government. The mission of this CNO was to better serve citizens who want to build, re-build, or re-use a house, factory, or barn, in the Netherlands. These citizens usually need to apply for licenses and permits regarding residency, spatial planning, and the environment. Each of these licenses and permits has their own set of criteria, procedures, administrative desks, waiting periods, fees, and staff. For both individual citizens and corporate citizens (i.e., companies), this is a complex and time consuming process that costs both applicants and the government a great deal of money. The Ministry for Housing, Spatial Planning and Environmental Management (VROM - initials in Dutch) wants to gather the different licenses together within the 'omgevingsvergunning' - the environmental permit. All aspects can then be requested from a single point and follow a single paid procedure to obtain a decision even if such decision needs the collaboration of different organizations.

The environmental permit project is part of a packet of measures that the Dutch cabinet has initiated to substantially reduce administrative charges for citizens and businesses. According to the Enviromental Licensing (General Provisions) Bill - WABO for its initials in Dutch, from January 1st 2009, municipalities, provinces and water board districts should be able to use the new process. The environmental permit is part of the modernization plan for VROM legislation, in which the ministry is reducing and improving its rules and regulations. The project includes development of an implementation plan with pilot projects and advice in different Dutch administrative regions. The third CNO we studied is one of these pilot projects.

The Netherlands is a parliamentary democratic constitutional monarchy divided into twelve administrative regions, called provinces. All provinces are divided into municipalities. The country is also subdivided in water districts, each governed by a water board, each having authority in matters concerning water management. The studied CNO is a networked organization among the province of Overijssel, the municipalities of Zwolle and Enschede, the water board district Regge \& Dinkel and Royal Grolsch N.V., a brewery (hereinafter referred to as Overijssel CNO), working in the WABO-ICT project. The aim of this project is to test the practical feasibility of the national online all-in-one service for environmental permits (LVO - initials in Dutch), steering the project on the services offered by IT to support the business processes.

The WABO forces the different public entities to cooperate with each other in a different way. This cooperation is possible only if it is supported by a correct provision of information. The WABO-ICT project investigates if both the process of cooperation and the support of an IS are feasible in practice. For testing the LVO, the Overijssel CNO used a situation where Royal Grolsch N.V. 


\subsection{CASE STUDY 3: REGIONAL GOVERNMENT NETWORK}

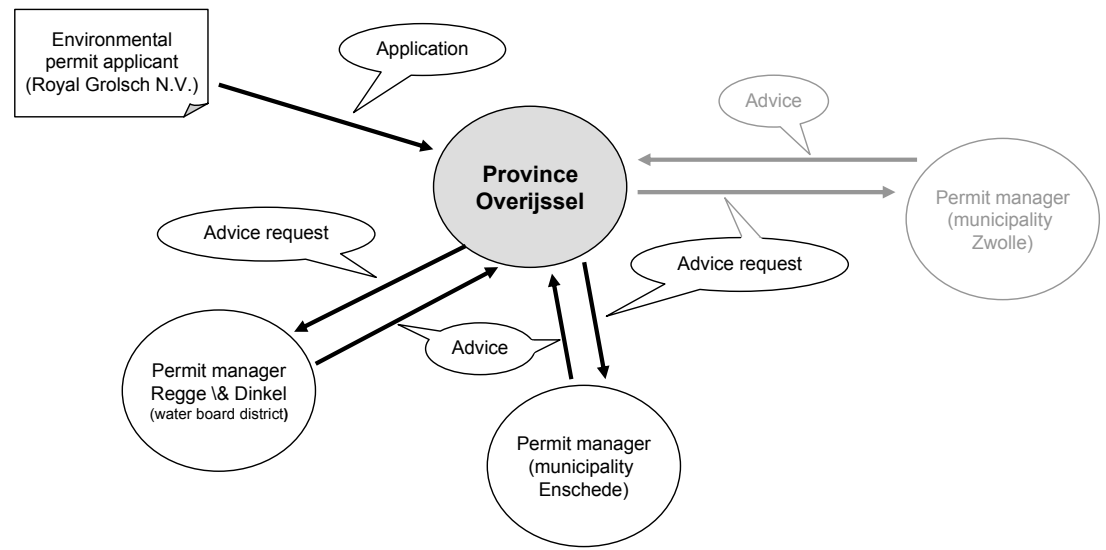

Figure 6.6: The To-Be state of the Overijssel CNO: Functional view.

plays the role of the 'client' asking for an environmental permit to the province of Overijssel. The province of Overijssel is the competent authority that asks advice (concerning residency and spatial planning) to the municipality of Enschede, and (concerning water issues) to the water board district Regge \& Dinkel as well (see Figure 6.6) because the province of Overijssel controls the environmental planning only. We make the note that for this particular case where Royal Grolsch N.V is physically located within the municipality of Enschede, the province of Overijssel would ask advice to this specific municipality only. The municipality of Zwolle will provide such advice just in those cases only in which the permit applicant is physically located within its territory.

\subsubsection{Research design and findings}

The middle layer in Figure 6.1 presents the research process we followed for this second case study. The Overijssel CNO provided us documentation concerning its collaborative work. We found two types of documents useful for the purpose of our case study and we carefully reviewed them. These were:

- Letters and memoranda concerning a variety of B-ITa topics in their collaborative work.

- Agendas, minutes and reports of meetings used to discuss alignment issues and solutions.

These documents were the only ones available to us. As all documents do, they could reflect the bias of the professionals who wrote them [348]. The documentation we obtained was mostly clear. However, there also were some unexplained acronyms and figures. To avoid confusion in our interpretations, we were able to ask our contact person in the Overijssel CNO. 
The primary research goal in this case study was to continue testing our initial hypothesis. Therefore, the form of document analysis we used was tracking. "Tracking is an easy-to-implement procedure compared to other forms of document analysis. However, its simplicity can also be a drawback when analysis or evaluation goals are more elaborate" [232, p. 37]. Generally, tracking involves testing a hypothesis by identifying "tracks" (i.e., facts) found in documents. That is, after the first refinement of the CSMO configuration in the first case study (see Figure 6.5), we had a notion about the B-ITa domains in CNOs and how they could work. Facts then were selected and analyzed from documents to find out whether the first refinement (and our notion) was correct. We present our findings in turn.

\section{Findings}

Partnering structure. It was clear that the definition of a blueprint of the Overijssel CNO was the first activity they did to begin to collaborate in order to know what was expected from each of them and to present how they fitted together. In general, the $\mathrm{CNO}$, as a networked organization in the public sector, had also a well-defined hierarchy of authority with powers and responsibilities understood by all, a clear-cut division of work among the participants and people, and an explicit set of procedures for making decisions. It was noted that increasing emphasis was being given to the balance of responsibilities. The major responsibilities in the WABO-ICT project were in the development of the technological architecture, the oversight of the IS, and the description of the collaborative processes and interfaces. One of the documents (with date close to the kick-start of the project) with information related to roles and responsibilities, made us to conclude that since the project began, there has been a clear indication of who was responsible for achieving specific goals, and what the role of each participating organization was. This resulted in a high commitment by all participating organizations. However, this commitment came from a specific individual goal of each participant: to be present and to contribute in a project (i.e., the WABO-ICT) that will have national impact in the future.

IS architecture. The documents analyzed revealed that the Overijssel CNO is highly dependent on ISs. We had access to different documents presenting the principles and norms they use for the development of the IS architecture, for example NORA [161], SOA, ISO 17799:2005. The Dutch Government Reference Architecture (NORA - initials in Dutch) is a set of models and principles showing how e-government works. They present the way in which it is possible to collaborate, to link processes smoothly to one another and to exchange data. In the Overijssel CNO, each of the three participating public organizations (i.e., the province Overijssel and the municipalities of Zwolle and Enschede) also has their own set of principles based on NORA. Such principles, together with the NORA, are the basis for the definition of specific principles and explicit requirements in the WABO-ICT project. Evidence from documents made us to understand that 


\subsection{CASE STUDY 3: REGIONAL GOVERNMENT NETWORK}

when the project started, they created a snapshot of the existing ISs in order to have a clear view of the current situation of the CNO concerning ISs they had, and they followed the same process to define its IS To-Be state. An identified problem was that the Overijssel CNO did not have any strategic innovation planning and it did not use any risk management techniques (at least, no document reported on these two IS activities). As a result, the selection of ISs in accordance with resources, needs and changing situations was deemed inadequate to minimize risks.

Process architecture. As in the IS architecture domain, a blueprint of the current and To-Be state of the collaborative processes was explicitly presented in some of the studied documents. To us, it was self-evident that the Overijssel CNO spent considerable time and effort in working sessions to design the choreography of all (individual and collaborative) processes needed to reach the goals of the CNO. The CNO took project management practices into account during the design of processes. For example, the allocation of human and technological resources was implicit in the process architecture design process. Documents reporting results of working sessions included (i) a workflow process that encompasses project approvals, checkpoint reviews, and periodic status reporting at project and statewide levels, and (ii) a plan to ensure that the work is done acceptably and that the project is in position to complete its phases successfully. Documents followed recognized best practices for project management (e.g., PMI practices as message/document-driven controls, scope statement definition and work breakdown structure). This was done with the goal to assure that the processes were properly performed and used in order to create the expected project deliverables. They relied on quality assurance.

Coordination. The Overijssel CNO tries to have highly rationalized activities resulting in simple and repetitive tasks. This situation leads to have a sharp division of work and to depend primarily on standardization of its processes and skills for coordination. Although standardization helps to coordinate the interdependencies among the participants, differences in meanings remain creating ambiguities. These ambiguities lead in turn to conflicts. Conflicts in the Overijssel CNO cannot easily be handled by informal communication since this kind of communication is sometimes held back by the standardization itself. Thus, they restrict the escalation of conflicts by direct supervision. The Overijssel CNO has appointed staff members who take the responsibility for the processes and provide instructions to others to monitor their work. The coordination team tries to minimize undesirable situations by organizing social events based on informal settings to encourage the synergy and the open discussion on, and exchange of new ideas of working together. They have created an organization that was more concerned about the service to be provided to citizens and its quality than about the political games that could arise. Coordination is usually achieved with communication and active information sharing. 


\subsubsection{Discussion}

The results in the Overijssel CNO partly replicated our previous findings. This CNO considered the partnering structure, IS architecture, process architecture and coordination [solution] as key areas to work on. IT infrastructure architecture was not a domain considered in their B-ITa efforts. However, in this case, we also identified a domain that was distinctive. It was quality assurance [solution]. They considered testing, verification and control as activities that need to be present in all projects. The quality team was consistently trying to assure quality in the BITa project we had access to. This team helped to plan activities to ensure that, for example, (i) the project employed all processes needed to meet requirements and (ii) the project meetings were effective without wasting time or efforts.

As CNOs are dynamic (see Chapter 2), they need to react quickly to customers needs and to run apace B-ITa projects in order to survive in a hypercompetitive environment. The Overijssel CNO was almost constantly analyzing its present IS architecture and how it helped to align strategies to the present situations. They used, for example, customer satisfaction analysis and market studies to define their B-ITa projects. They react fast, but before 'doing', they take considerable time in discussing, planning and analyzing such projects (see latest mechanism in Figure 6.7). Our findings in this case study site replicated the results presented by de Koning and van der Marck where they show how "no case, no go" [80, p. 45] (i.e., if there is no positive fact-based explanation of the reasons for investing in IT, no action should be taken) is the motto of the most succesful businesses in the Netherlands for B-ITa-related decisions. It is to avoid unfavorable future results.

The findings of this case partially increased the strength of our original claim: B-ITa improvements [outcome] are caused by addressing aspects related to partnering structure, IS architecture, process architecture and coordination [solution]. However, quality assurance [solution] seemed to be also an instrument to promote improvements in B-ITa, and we found that swift-reacting but in a delayed form [mechanism] is a strategy to follow when achieving B-ITa in CNOs. In the Overijssel CNO, the partnering structure, quality assurance and coordination domains created an ideal environment to work smoothly in the IS architecture and process architecture domains, which in turn improved its B-ITa. These ideas enriched the understanding of our own claim, helped to refine our CSMO configuration (see Figure 6.7), and led us to continue looking for evidence.

\subsection{Case study 4: Provincial government relation}

The next CNO we analyzed was a network of provincial government agencies in Canada, namely, the Ontario Council of Agencies Serving Immigrants (see www.ocasi.org), hereinafter referred to as the Ontario CNO. It was formed in 1978 and today includes around 200 partnering organizations. According to Canada's government decentralization concept, a council is a registered charity governed 
6.5. CASE STUDY 4: PROVINCIAL GOVERNMENT RELATION

\begin{tabular}{|c|c|c|c|c|c|c|}
\hline Context & + & Solution & + & Mechanism & $=$ & Outcome \\
\hline \multirow{6}{*}{$\begin{array}{l}\text { Technology outsourcing } \\
\text { felation between } \\
\text { organizations in the private } \\
\text { sector } \\
\text { State government } \\
\text { collaboration-ameng severat } \\
\text { departments } \\
\text { including provincial and } \\
\text { municipal agencies }\end{array}$} & & Partnering structure & \multirow{6}{*}{$+<$} & \multirow{2}{*}{$\begin{array}{l}\text { Random considera- } \\
\text { tion of the domains }\end{array}$} & \multirow{6}{*}{$=$} & \multirow[b]{2}{*}{$\begin{array}{l}\text { B-ITa } \\
\text { (improvements) }\end{array}$} \\
\hline & & IS architecture & & & & \\
\hline & & Process architecture & & High social status of & & \\
\hline & & $\begin{array}{l}\text { Coordination } \\
\text { Gost management }\end{array}$ & & $\begin{array}{l}\text { automated ISs to } \\
\text { support the collabo- } \\
\text { rative processes }\end{array}$ & & \\
\hline & & $\begin{array}{r}\text { IT infrastructure } \\
\text { arehitecture }\end{array}$ & & $\begin{array}{l}\text { Engagement in a } \\
\text { 'swift-reacting but in }\end{array}$ & & \\
\hline & & Quality assurance & & strategy & & \\
\hline
\end{tabular}

Figure 6.7: Second refinement of the CSMO configuration.

by a voluntary board of directors elected by the member agencies. The council reports to both the federal government of Canada and the province of Ontario. The purpose of the council which we study is to act as a mediator between the immigrant-serving agencies in Ontario and the federal government, and to coordinate responses to the shared needs and concerns of the settlement sector. The 200 member agencies forming the so-called 'settlement sector' provide some services to newcomers settled in the province of Ontario. Examples of types of such agencies are: Language Instruction for Newcomers to Canada (LINC) centers (more information in http://www.eslincanada.com/linc_programs.html), the YMCA newcomers centers (http://www.ymcatoronto.org/en/newcomers/index.html), urban services organizations, and francophone centers.

In 2004, the Ontario CNO initiated a large, settlement-sector-wide, project of aligning its service-delivery-processes and IT [63]. It aimed at building a flexible, efficient and responsive information management system shared by the agencies in the Ontario's settlement sector. The goal of the system, called Settlement Sector Client Administration Network (SSCAN) system, was to standardize and harmonize the service-provisioning and reporting processes across the agencies within the Ontario CNO. It was scheduled to be fully operational in April 2009. The service provisioning processes being automated by means of SSCAN are shared among the sector itself and its funders (those who provide money for its existence), namely, Citizenship and Immigration Canada (CIC - a ministry at the federal level), Ontario's Ministry of Citizenship and Immigration (MCI), and Ontario's Women's Directorate (OWD).

The shared system enables the participating government agencies to contribute - each in its own way, to the CIC mission of "developing and implementing policies, programs and services that facilitate the arrival of persons and their integration into Canada in a way that maximizes their contribution to the country while protecting the health, safety and security of Canadians" (www.cic.gc.ca). To present the scope of this B-ITa initiative, we make the note that the Ontario CNO welcomes per year $50 \%$ of all immigrants to Canada (that is, 130000 people 


\section{CHAPTER 6. B-ITA DOMAINS: EMPIRICAL VALIDATION}

settle in the province of Ontario and become new clients of some agencies of the settlement sector). It is also important to note that the 200 partnering agencies are diverse in terms of (i) the services they provide, (ii) the clients they serve, (iii) their regional contexts, and (iv) their missions. For example, Ontario's MCI classifies them as 'small and large', as 'single-service and multi-service' and as 'ethno-specific and general'. For responding to diversity of participating agencies, the shared system supports each agency in creating and managing its own service program (hence, identifying and managing its own information and reporting needs).

Prior to the start of the project, the agencies of the Ontario CNO carried out a study on the information management challenges they faced [63]. They identified the following reasons for the low level of efficiency in their service delivery processes: tripled data-entry, separate systems, electronic and paper record keeping, all of which added up to labor-intensive reporting to CIC, MCI and OWD, to slowed-down service provisioning and to frustrated font-line staff. The Ontario CNO considers the SSCAN project as the solution to let them provide better services to newcomers by using a central information repository and a common system for managing client cases [63]. The expected outcome is the enhanced productivity of the settlement agencies and their increased capacity to deliver services.

\subsubsection{Research design and findings}

The lower layer in Figure 6.1 presents the research process we followed in the Ontario CNO case. In this case study, data was collected solely based on publicly available resources published in Canadian government web sites (examples are presented in Table 6.2). The documents contained information of a province-wide large initiative to build a flexible, efficient and responsive information management system shared by all government agencies making up Ontario's settlement sector. Our choice for reviewing these documents was motivated by the fact that Canada's government agencies publish project documentation with level of detail suitable for a documentary case study. We judged suitability in terms of (i) the broad variety of types of documents made publicly available to the citizens, (ii) the broad variety of perspectives involved, e.g. those of the participating government agencies, the consultants who assist them in their B-ITa analyses, the government alignment consulting companies, (iii) the broad variety of illustration examples being provided in the documents, which let us abstract high-level notions and map them to concrete instances of B-ITa phenomena relevant to our investigation $^{2}$.

Similarly to the case study presented in Section 6.4, the form of document analysis we used in this study also was tracking. The documents, which we deemed valuable to include in our study, fall into two categories (see Table 6.2):

\footnotetext{
${ }^{2}$ The public availability of these documentation sources is a consequence of the federal, provincial, and municipal governments' commitment to a high level of transparency in how tax-payers' money gets spent on IT and on service improvement initiatives.
} 


\subsection{CASE STUDY 4: PROVINCIAL GOVERNMENT RELATION}

those describing the initial situation - i.e., the (pre) B-ITa state - and those referring to the To-Be state. As Table 6.2 indicates, the documents were of various types, ranging from business requirements of shared applications and business cases, to qualitative research being commissioned by the government to get the initial situation clarified and understood by all stakeholders. The variety of the information sources offered a variety of perspectives on how to reason about B-ITa success domains. The documents are authored by government stakeholders, by standardization bodies, by government consulting service companies (for example the Commons Group - see www.commons.ca), and by researchers.

Table 6.2: Documents studied in the Ontario CNO case.

\begin{tabular}{|l|c|c|}
\hline Document & As-Is & To-Be \\
\hline Study of settlement sector database needs [63] & $\times$ & \\
\hline $\begin{array}{l}\text { Results of "extensive consultation with agencies and in-depth re- } \\
\text { views of IS" [63, pp. 31-41] }\end{array}$ & $\times$ & \\
\hline Current systems [63, pp. 45-54] & $\times$ & $\times$ \\
\hline Case management system requirements [63, pp. 42-44] & $\times$ & $\times$ \\
\hline $\begin{array}{l}\text { Immigration-contribution accountability measurement sys- } \\
\text { tem [55] }\end{array}$ & & $\times$ \\
\hline Solution proposals for a shared system [63, pp. 17-30] & $\times$ & $\times$ \\
\hline Service Mapping [101] & & $\times$ \\
\hline Business case for the shared system [63, p. 30] & & \\
\hline $\begin{array}{l}\text { Settlement Service standards - inventory of work-in-progress and } \\
\text { future steps [137] }\end{array}$ & $\times$ & $\times$ \\
\hline Evaluation of technology investments [162] & $\times$ & $\times$ \\
\hline Models of Settlement Service Workshop [223] & $\times$ & $\times$ \\
\hline Re-visioning the Newcomer settlement support system [287] & $\times$ \\
\hline
\end{tabular}

\section{Findings}

Partnering structure. As a member of a council of autonomous communitybased agencies, each agency in this CNO has a clearly defined role and responsibilities. The division of work among agencies is transparent and is traceable to the specific services which each agency is specialized in. Within the CNO, the partnering agencies are in a well-defined relationship, which includes "(i) a commitment to mutual goals, (ii) a jointly developed structure and shared responsibility, (iii) mutual authority and accountability for success, and (iv) sharing of resources and rewards" $[82$, p. 136]. Based on the roles that the Ontario CNO plays at the various committees, different information must be made easily available for performance reporting and tracking as well as management decision making (for example, the decision on launching new services or on shifting responsibilities from one type of agencies to another). To the CNO, "clarity in discussing the differing roles and responsibilities of partners is essential to the task of developing new models of settlement services and the systems which support the service provisioning processes" [223]. The documents, which we reviewed, indicated that 


\section{CHAPTER 6. B-ITA DOMAINS: EMPIRICAL VALIDATION}

in addition to the partnership's meaning as an organizational foundation for service delivering, the concept of partnership within the $\mathrm{CNO}$ also encompasses five other meanings: (1) consultative (i.e. advisory), (2) contributory (i.e. managing shared funds), (3) merger/takeover (of agencies), (4) operational (i.e. delivery of work that is purchased), and (5) collaborative (i.e. real sharing of power) [223].

IS architecture. The IS architecture of the SSCAN project was analyzed by the CNO at the stage of problem analysis and business/IT requirements definition [63]. This all was done by using three reference models [101], each referring to a specific level of government, being municipal, provincial and federal. These reference models helped the collaborating government agencies define universal definitions for terms regarding their shared programs services, public needs, and outcomes [101]. It was used to map and produce a picture of each service-delivery

process and the applications used in its support. For example, one outcome of the analysis of the current IS architecture was the realization that within the CNO there were 11 different systems with duplicating case management functionality being used by individual member agencies. The documents we reviewed also indicated the IS architecture as key for evaluating solution implementation options. The CNO considered three different ways to implement the SSCAN system: distributed, centralized and hybrid [63, p. 22-25]. The recommendation, which came out of comparing the three options, referred to the hybrid approach as the best way to go. It included a centralized technology and distributed control, which was aligned with the features of the partnering structure. This option included the development of two centralized web-based systems - one to be used by agencies and one by the funders.

Process architecture. The participating government agencies in the Ontario CNO analyzed their current cross-organizational service provisioning process and the To-Be processes supported by means of the SSCAN system. An important quality aspect of the process architecture in this CNO is that for some processes, multiple channels of service provisioning must exist. For example, certain services (or certain parts of them) should be delivered equally well by personal counseling, telephone counseling, and e-counseling. The new system supports the service delivery process independently of the channel through which it happens. We found that this feature of the process architecture led to the choice to keep the shared SSCAN system open and inclusive to any agency-specific application which a CNO-partner might deem important in supporting its local process architecture. The documents we reviewed emphasize that the CNO plans to allow certain variability in terms of cross-organizational processes which compose the shared process architecture. For example, SSCAN will not be mandatory for those agencies whose existing ISs already have the capacity to meet funder requirements and exchange data with SSCAN. These agencies are welcome to keep variety in terms of service-provisioning processes and client information management processes but they should know why they do it and why their processes vary. 


\subsection{CASE STUDY 4: PROVINCIAL GOVERNMENT RELATION}

Coordination. The Ontario CNO makes a explicit distinction between horizontal and vertical coordination forms and is well aware of their implications on coordinated service provisioning. Horizontal coordination refers to agencies at one specific level of government (e.g. either municipal or provincial). Vertical coordination refers to coordinating service provisioning actions across institutions at all three levels: municipal, provincial and federal. Partnering agencies also made a clear distinction among "cooperation, coordination and collaboration" [82, p. 136]. They acknowledge that while these three are "functionally related, they are quite different and distinct from each other" [82, p. 136] in terms of the level of commitment to mutual relationships and goals, the level of formality in specifying the shared responsibility, the level of mutual authority and accountability for success, and the level of sharing of resources and rewards. The Ontario CNO made an inventory of where coordination takes place $[82$, p. 80$]$ and their implications for service coordination. The inventory identified six forms: (i) "coordination within a specific service sector" (example: among agencies providing language learning services), (ii) "coordination between two of more service sectors" (example: between language learning services and employment services), (iii) "coordination around a particular service program" (example: around a pre-school program), (iv) "coordination around a particular social problem" (example: alcoholism), (v) "coordination among organizational functions" (example: client intake, service delivery, marketing), and (vi) "coordination around services for a specific client group" (example: around services for children with disabilities).

\subsubsection{Discussion}

The findings in the Ontario CNO replicated the earlier two case studies in that partnering structure, IS architecture, process architecture and coordination [solution] are the pillars in the Ontario CNO alignment initiative. What we found unique in this case is the bold emphasis on coordination, (i) both vertical and horizontal, and both (ii) within agencies of the same type and cross-agency coordination happening around specific service programs. The documents we reviewed revealed that the Ontario $\mathrm{CNO}$ considered the so-called 'sectoral service standards' $[42,43,137]$ as prerequisites for their alignment project success. These (settlement) service standards are a checklist against which the participating government agencies review and evaluate their programs. The standards are also used by the agencies as the basis for developing their own service manuals. Moreover, we found evidence which suggested that the intent of standards is to enforce service quality assurance policies. For example, the following piece of text from the Ontario's government website about the goals of the service standards strengthens this suggestion: "Service standards are goals that we should try to achieve. They can help us give better service because they invite feedback from the public. And they can be applied to almost every service we deliver in the federal government, from issuing a license to managing an internal operation that supports front-line workers. Establishing standards for such things as quality, frequency and cost can help improve a broad range of federal services." 


\section{CHAPTER 6. B-ITA DOMAINS: EMPIRICAL VALIDATION}

Furthermore, the Ontario CNO assures the quality of its service coordination models by means of a shared interoperability framework (SIF). SIF is about municipal-provincial-federal interoperability and is defined as "the structured set of de jure standards, de facto standards, specifications, and policies allowing computer systems to interoperate" [59, p. 14]. It was found to be "a necessary condition for accomplishing the goals of online government, for controlling costs in certain sectors (such as health), and for providing new services to citizens". The evidence brought us to conclude that with respect to the importance of quality assurance [solution], the Ontario case replicates this finding from Overijssel.

\subsection{Cross-case analysis}

From the cumulation of ideas, the replication of findings in the three case studies and the CSMO configuration refinements (see Figures 6.5 and 6.7), we propose only four B-ITa domains to be considered by CNOs in their efforts for achieving B-ITa:

- partnering structure,

- IS architecture,

- process architecture and

- coordination.

Despite of the variation in the findings of the three case studies, the ICoNOs MM includes only these four B-ITa domains. We did not consider 'cost management', 'IT infrastructure architecture' and 'quality assurance' as B-ITa domains because our findings concerning such domains are not replicated in the single cases. Clearly, one might argue that these, and even more domains, must also be addressed when striving for B-ITa in CNOs. However, we were looking for the most logical division of concerns, and the most frequent way of working (collaboration pattern), with respect to B-ITa in CNOs. That is,

our four B-ITa domains represent the minimal number of domains that the CNOs we studied take into account to achieve B-ITa. Generalizing, they are the domains that one can distinguish when improving CNO B-ITa. They then are that most logical division of concerns, and that most frequent way of working, with respect to B-ITa in CNOs.

As our findings suggest, there are however additional domains that might be considered in B-ITa improvement attempts in certain context, but are not present in all B-ITa initiatives. For example, an additional condition for achieving B-ITa in CNOs would be cost management (as found in the first case study presented in Chapter 5). However, while managing costs could be important, it is by no means necessary for achieving B-ITa in every single CNO B-ITa project. 


\subsection{CROSS-CASE ANALYSIS}

Our hypothesis (see Section 6.2) was partially confirmed. That is, after conducting the three case studies presented in this chapter, we claim that in any successful B-ITa improvement process in a CNO, improvements in partnering structure, IS architecture, process architecture, coordination (only) cause more improvements of B-ITa than improvements in other domains do.

In order to identify similarities and differences in the degree of formalization of the four B-ITa domains, we furthermore analyzed data across the three cases. By identifying similarities and differences, we provide further insight into the issues concerning B-ITa by generalizing the case study results.

\subsubsection{Solution: the B-ITa domains}

\subsubsection{Partnering structure}

The partnering structure in the Tamaulipas CNO was well-defined. However, they had a distribution of authority which did not match the required distribution of work. This situation, according to our findings, is an obstacle to proper B-ITa. In this case the partnering structure domain was crucial to improve B-ITa. On the basis of the Tamaulipas CNO case, we can observe that more obstacles in the partnering structure may exist in other cases. Therefore, already from this case we can generalize that partnering structure consistently must be attended to when improving B-ITa because it can contain critical obstacles towards improving B-ITa. The results of the Overijssel CNO and the Ontario CNO cases support such generalization. In these cases such a partnering structure definition was important when collaborating. We, however, found an interesting distinction between the partnering structure in the latter two settings: while the Overijssel case emphasized the importance of a well-defined hierarchy of authority with powers and responsibilities to standardize, the Ontario case emphasized "the space for diversity" [63, p. 19] and the network's philosophy and lived commitment to actively promote diversity. For example, in their experience, "using standards to actively support diversity has proven helpful" in dealing with "IT-vendor competition and in encouraging the development of innovative custom solutions for agencies with unique needs" [63, p. 19]. The Ontario CNO emphasized that only when it left space for diversity, the CNO could be successful. In both cases, each CNO claimed that without the distinctive characteristic of the partnering structure, their B-ITa improvement efforts had not had positive results.

\subsubsection{IS architecture and process architecture}

As we explain in our definition of B-ITa (see Chapter 2), the business requirements come out from the analysis of the CNO goals and processes. In the three case study sites (Tamaulipas, Overijssel and Ontario), these requirements were definitely reflected in both the IS architecture and the process architecture. These observations let us conclude that, if a $\mathrm{CNO}$ is not mature enough with respect 
these domains - i.e., IS architecture and process architecture - (though with mature partnering structure and mature coordination), the B-ITa maturity of the entire CNO can not be high. In the Tamaulipas CNO case, process architecture was attended to routinely. Its process architecture domain was consistently attended. When analyzing the data, we could explicitly see the IS architecture and the process architecture in the foreground and we consider a possible explanation for this the matter that government agencies concentrate their attention more on procedures and on applications than on other domains (as cost management or quality assurance).

\subsubsection{Coordination}

The results of the case studies converge on that coordination is also an important domain to consider when achieving B-ITa. Commonly, government agencies depend on standardization of processes and skills for coordination [134, 198]. For example, the Overijssel CNO and the Ontario CNO use respectively the NORA and the SIF frameworks to standardize their IT environment across the different participants. However, we also have found some mechanisms that can be an obstacle for proper coordination in a CNO. For example, the differences in meaning between the participating organizations with respect to a particular work situation diminish the importance of standardization. This diminishing mechanism is also an obstacle for a mature B-ITa, and leads us to conclude that one has to be aware that more coordination obstacles can appear in CNOs. In the Tamaulipas $\mathrm{CNO}$ and the Overijssel CNO, both communication and information sharing have helped to maintain a proper coordination. From our studies, we conclude that coordination must be addressed in CNOs considering active communication and active information sharing as supporting mechanisms.

\subsubsection{Mechanisms}

\subsubsection{Relations between the B-ITa domains}

The relations between the B-ITa domains assert that having a well-defined collaborative work structure as a basis for the definition of the architecture of ISs and of the process architecture, helps the CNOs to react promptly to the business needs. This is in a situation where coordination mechanisms are present to manage the interaction and work among the CNO participants. This understanding is reflected in Figure 6.8. The figure presents both a sequence of relations (namely that partnering structure and coordination precede IS architecture and process architecture - see timeline), and a dependency relation (see dotted arrow) between the four B-ITa domains. These two kinds of relations structure the four B-ITa domains and help explain how the CNOs we studied achieve B-ITa by considering the four domains.

Regardless of how a CNO defines its partnering structure, the IS/process architects in the CNO must (i) consider such a structure for the design of the IS 
6.6. CROSS-CASE ANALYSIS

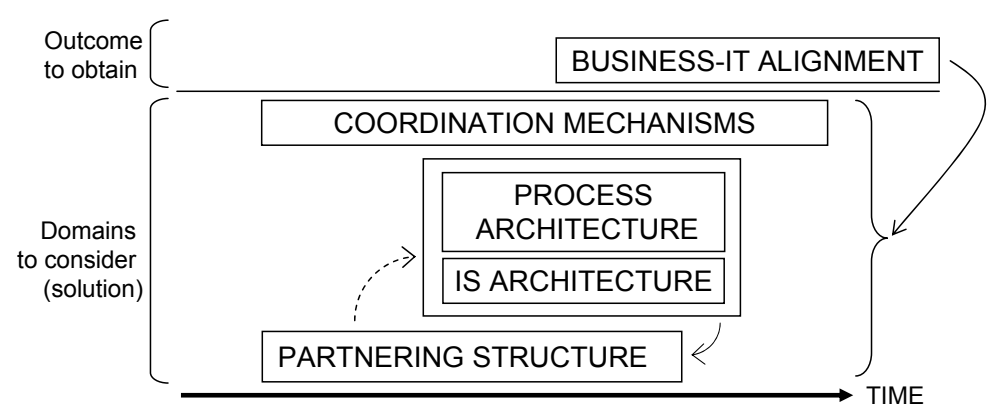

Figure 6.8: Relations between the B-ITa domains in CNOs.

architecture and process architecture, and (ii) make sure that a good communication exists between those who design the partnering structure and the teams involved in the IS architecture and process architecture domains. This is necessary because the partnering structure design produces constraints that need to be met by the IS architecture and process architecture designs. This is explicitly evident in the Ontario case study, in which the CNO rejected those solution options which did not respond to the partnering structure requirements. However, we must acknowledge that in the Ontario case study, the CNO's partnering structure pertains to 200 partners, while the number of partners in the other two case studies was significantly lower.

The solid arrow starting from the IS architecture and process architecture domains and pointing to the partnering structure in Figure 6.8 asserts that it is possible to first design an IS architecture and later re-adjust the design of the partnering structure in a way that ensures the crucial fit between the structure and the IS architecture. Then, later it may be possible to check whether the constraints/requirements resulting from this partnering structure mandate some adjustment in the IS architecture. This arrow represents a feedback loop that plays an important role in the enhancement of the B-ITa domains when achieving B-ITa. We must note, however, that the Ontario CNO rejected the route to first design the IS architecture and later re-adjust the design of the partnering structure. Instead, the Ontario CNO believed that if an IS architecture is designed around a common database and a solution which fit the partnering structure, this can, later on, have a positive impact in that it will make other government agencies at federal level consider aligning to the Ontario CNO's IS architecture. The motivation for this is that when 200 provincial organizations must report data to one other agency at federal level, it will be much cheaper that this one agency aligns its reporting requirements to the reporting standard of the 200 partners, rather than the other way around.

Finally, the remaining arrow starting from the "outcome to obtain" side and pointing to the "domains to consider (solution)" side in Figure 6.8 represents a feedback process. This feedback process is to improve and evolve the four domains through experience. When achieving B-ITa, measurement and analysis processes help to define measures (e.g., performance measures as it is in the Ontario CNO 


\section{CHAPTER 6. B-ITA DOMAINS: EMPIRICAL VALIDATION}

and the Overijssel CNO, or financial and internal measures) and communicate the B-ITa results in order to consider them in future B-ITa projects to assure quality and real improvements. For example, in the Ontario case, a specific performance assessment framework, namely the Contribution Accountability Framework [55], has been used for this purpose.

\subsubsection{Additional mechanisms}

We have also found additional outstanding mechanisms that might be considered for B-ITa improvements in CNOs (see Figure 6.7). These mechanisms are:

- Random consideration of the B-ITa domains.

- High status of IT in the CNO and automated ISs to support the collaborative processes.

- Engagement in a 'swift-reacting but in a delayed form' strategy.

We believe that these three mechanisms explicate the logic of the B-ITa domains, i.e. they describe interventions that bring about B-ITa (improvements) or they present ways in which the B-ITa domains may be permeate into the reasoning of the team that works for improving B-ITa.

\subsubsection{Context}

We make the explicit note, that because the case studies presented in this chapter have been conducted in CNOs formed by government agencies (that is, the context in the realist approach), it can be argued that our results can not be brought up in the private sector. Research (e.g., [19, 35, 27, 248, 291]) analyzing the differences between public and private organizations will support such an argument. However, these authors have also found similarities between the two sectors. Likewise we have also found evidence, in our case studies, that confirms some similarities. Therefore, we can conclude that our results are not clear-cutlimited to the public sector. We believe that CNOs in both sectors begin a B-ITa project with a solid mission statement that drives the strategic planning process to meet the common goal(s). This mission reminds the participating organizations of their work principles and respective roles in the network. We have studied entrepreneur-led (see Chapter 5) and governmental CNOs where (i) participants pool costs, skills, and core competences to provide world-class solutions (products or services) that could not be provided by any of them individually; (ii) ISs are able in each of the participants to respond dynamically to meet the ever-changing customer needs and to communicate and share information among them; (iii) participants have a clear understanding of the common goal(s) and the functions of each of the participating organizations in order to know what is expected from each of them. Such characteristics are reflected in our definition of CNO (see Chapter 2). So, both sectors are similar. Only their purpose could vary. And, this difference in intention creates the environment in which they operate and how they do it. 
From a CSMO configuration perspective, the general context where our BITa domains [solution] work for having B-ITa improvements [outcome] is a group of organizations, connected by IT, that work together to accomplish tasks, reach common goals and serve customers over a period of time where the characteristics (i), (ii) and (iii) introduced in the previous paragraph are present. We however make the explicit note that our B-ITa domains [solution] can 'work' in quite separate ways according to the context of the $\mathrm{CNO}$, and that our B-ITa domains could be implemented in different ways and that they might be more effective with (or could have more use in) some CNOs rather than others - for example, although we believe that our domains apply for CNOs from both the private and the public sectors, it might be that they are more suitable for collaborating government agencies. Using our case studies, we synthesized the best evidence available without pretending that our study covered every conceivable mechanism, process or outcome. We therefore acknowledge the fact that more research is needed to identify additional mechanisms that could affect the use of the final B-ITa domains included in the ICoNOs MM.

\subsection{Summary}

In this chapter, we presented the three case studies that we have conducted to empirically validate the B-ITa domains found after using a focus group and a first exploratory case study (see Chapter 5). Based on our findings, we propose to consider four key domains for successful B-ITa in CNOs:

- partnering structure,

- IS architecture,

- process architecture and

- coordination.

We claim that these domains are the domains to consider when improving B-ITa in CNOs. A new responsibility for CNOs involved in B-ITa projects is focus on these domains to obtain new insights and to strive for B-ITa maturity. Although we have found additional domains to be addressed in B-ITa improvement efforts, the ICoNOs MM includes only the four domains listed above based on the cumulation of ideas, the replication of findings in the three case studies and the CSMO configuration refinements (see Figures 6.5 and 6.7).

For each of the case studies, we introduced (i) the site we investigated, (ii) the research design and findings, and (iii) a discussion of results in order to identify BITa domains and mechanisms to improve B-ITa. Additionally, in order to provide further insight into issues concerning B-ITa by generalizing the case study results, we presented a cross-case analysis.

We believe that our results are not specific to CNOs in the public sector only. As we have conducted our cases in three CNOs formed by government agencies, we indeed consider our results to be useful for the public sector. However, the results are not clear-cut-limited to this sector. After conducting the case studies 
presented in this chapter, we found some similarities between public and private organizations that support generalization of our results. 


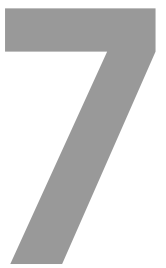

B-ITa processes

\subsection{Introduction}

In this chapter, we focus on the POPULATE step of the MM development process presented in Section 4.2.1 of Chapter 4. After defining the levels and the domains of our MM (Section 4.2.2 of Chapter 4, and Chapter 6), it is required to populate the model with process areas. That is, one needs to identify the process areas to include in each of the domains at each maturity level. A process area is a group of practices in a domain which, when implemented collectively, satisfy goals considered important for making an improvement in that domain.

This chapter introduces the first version of the ICoNOs MM (Section 7.2). Furthermore, we present the literature survey (Section 7.3) and the case study (Section 7.4) conducted to, respectively, identify the process areas included in each of the B-ITa domains (see Chapter 6) and to confirm the utility of those process areas in a real-life CNO (and therefore, to support their inclusion in our model). Based on the results of this case study, we found fifteen process areas that could be clustered in eight B-ITa best practices (Section 7.4.3) that can be drawn on by CNOs. Finally, Section 7.5 summarizes this chapter.

Preliminary versions of Section 7.3 and Section 7.4 have been published in the proceedings of the International Workshop on Enterprise Interoperability [275], the 15th International Conference on Concurrent Enterprising [268] and as a Centre for Telematics and Information Technology Technical Report [277], respectively.

\subsection{The ICoNOs MM: The first version}

The first version of the assignment of process areas to the B-ITa domains at the five levels is presented in Figure 7.1.

It can be argued that the positioning of the processes into a specific B-ITa level seems arbitrary. However, the decisions for such a positioning were driven by the definition of each process (see Section 7.2.1) and by the observations in the three case studies we conducted to validate the design of the ICoNOs MM (see 
CHAPTER 7. B-ITA PROCESSES

\begin{tabular}{|c|c|c|c|c|}
\hline & \multicolumn{2}{|l|}{ PARTNERING STRUCTURE } & \multicolumn{2}{|l|}{ IS ARCHITECTURE } \\
\hline 5 & & & Risk analysis and mitigation & RAM \\
\hline 4 & Metric-based roles exploration & MRE & Quantitative IS portfolio management & QPM \\
\hline 3 & $\begin{array}{l}\text { Governance structure and compliance } \\
\text { Roles and responsibilities specification } \\
\text { Inter-organizational policies definition }\end{array}$ & $\begin{array}{l}\text { GSC } \\
\text { RRS } \\
\text { loPD }\end{array}$ & $\begin{array}{l}\text { IS architecture target formulation } \\
\text { IS capabilities definition } \\
\text { IS architecture verification and validation } \\
\text { IS portfolio management }\end{array}$ & $\begin{array}{l}\text { ATF } \\
\text { IsCD } \\
\text { AVV } \\
\text { IsPM }\end{array}$ \\
\hline 2 & $\begin{array}{l}\text { Business model definition } \\
\text { Service level agreements definition } \\
\text { Organizational structure definition }\end{array}$ & $\begin{array}{l}\text { BMD } \\
\text { SLA } \\
\text { OSD }\end{array}$ & $\begin{array}{l}\text { Baseline IS architecture description } \\
\text { Standards and principles definition } \\
\text { IS requirements management. }\end{array}$ & $\begin{array}{l}\text { BAD } \\
\text { SPD } \\
\text { IsRM }\end{array}$ \\
\hline
\end{tabular}

\begin{tabular}{|c|c|c|c|c|}
\hline & \multicolumn{2}{|l|}{ PROCESS ARCHITECTURE } & \multicolumn{2}{|l|}{ COORDINATION } \\
\hline 5 & $\begin{array}{l}\text { Inter-organizational process optimization } \\
\text { Causal analysis and resolution }\end{array}$ & $\begin{array}{l}\text { IoPO } \\
\text { CAR }\end{array}$ & & \\
\hline 4 & $\begin{array}{l}\text { Organizational process performance } \\
\text { Event logs formal consistency }\end{array}$ & $\begin{array}{l}\text { OPP } \\
\text { EFC }\end{array}$ & Quantitative coordination relation analysis & QRA \\
\hline 3 & $\begin{array}{l}\text { Organizational process focus planning } \\
\text { Process architecture target formulation } \\
\text { Process architecture definition } \\
\text { Process portfolio management }\end{array}$ & $\begin{array}{l}\text { PFP } \\
\text { PAF } \\
\text { PAD } \\
\text { PPM }\end{array}$ & $\begin{array}{l}\text { Standardization } \\
\text { Communication-oriented coordination }\end{array}$ & $\begin{array}{l}\text { STD } \\
\text { COC }\end{array}$ \\
\hline 2 & Baseline process architecture description & BPD & $\begin{array}{l}\text { Informal communication adjustment } \\
\text { Direct supervision }\end{array}$ & $\begin{array}{l}\text { InCA } \\
\text { DTS }\end{array}$ \\
\hline
\end{tabular}

Figure 7.1: ICoNOs MM: The first version.

Chapter 6). That is, at maturity level:

2: Isolated, we positioned those processes that are the basic practices to support a particular B-ITa domain. They are isolated initiatives that are not managed from the entire CNO perspective.

3: Standardized, we positioned those processes that are directed to make B-ITa domains management improvements. They are performed from a CNO perspective (i.e., they need to be cooperative initiatives in order to be effective).

4: Quantitatively Managed, we positioned those processes where CNOs must use statistical and other quantitative techniques.

5: Optimized, we positioned those processes that are focused on continuously optimizing the range of process performance through both incremental and innovative improvements.

As we have explained in Section 4.2.2 of Chapter 4, level 1: Incomplete, contains no process areas because, in our ICoNOs MM, at this maturity level processes related to a particular B-ITa domain are usually not performed or partially performed. That is, such a particular domain is not explicitly considered when a CNO strives for B-ITa. 
In the following, we present the first set of process areas included in the ICoNOs MM (see Figure 7.1) in alphabetical order, grouped into the four B-ITa domains. For each process area, we state what it means and provide (in parentheses) the level in which the process area is positioned. Detailed explanation of the B-ITa process areas can be found in Chapter 8 .

\subsubsection{First set of B-ITa process areas}

\subsubsection{Partnering structure}

BMD Business model definition (L2). To define a blueprint of how the CNO works, describing how different variables of the collaboration fit together as a system to help creating value for each participant.

GSC Governance structure and compliance (L3). To structure the priorities and allocation of resources and decision rights to create accountability; and to ensure that activities are performed in conformity with policies and procedures. A successful compliance process will be performed through definition of effective policies and procedures.

IoPD Inter-organizational policies definition (L3). To define the policies intended to influence and determine decisions, including shared risk and rewards policies to increase mutual benefits perception and shared commitment.

MRE Metric-based exploration of roles (L4). To employ approaches as relational exchange techniques, organizational communication's mechanistic and system-interaction methods to study organizational communication, structure and roles in the collaboration.

OSD Organizational structure definition (L2). To define the cross-organizational ties constructing a framework for cross-organizational decision making and placing power and authority in order to regulate the CNO work.

RRS Roles and responsibilities specification (L3). To specify the roles and responsibilities, and their related guiding principles, of the participants in the CNO after defining its organizational structure.

SLA Service level agreements definition (L2). To describe the agreements on the deliverables, quality, and fitness-for-purpose of services that have an impact on the work of each participating organization. A successful implementation of these agreements will be delivered through effective governance structure.

\subsubsection{IS architecture}

ATF IS architecture target formulation (L3). To evaluate, select and design ISs needed to support the desired To-Be state of the IS architecture taking into account business and IT drivers, and the processes to support. 


\section{CHAPTER 7. B-ITA PROCESSES}

AVV IS architecture verification and validation (L3). To perform periodically gap analysis to make sure changing IS requirements are managed in consistent fashion with IS architecture targets. A successful verification and validation will be performed through an effective IS target formulation.

BAD Baseline IS architecture description (L2). To create a snapshot of the existing ISs and data, assessing what the current state of the CNO is concerning ISs.

IsCD IS capabilities definition (L3). To define the ability of the collaboration to achieve new forms of competitive advantage by ISs in order to achieve congruence with the business environment where it works.

IsPM IS portfolio management (L3). To create the right mix of ISs investments to properly use limited resources while providing the maximum business benefit. A successful IS portfolio management will be delivered through the execution of the other IS processes effectively.

IsRM IS requirements management (L2). To manage the changing IS requirements during their engineering process and the development of the required ISs.

QPM Quantitative IS portfolio management (L4). To use quantitative techniques to analyze, assess, and control IS portfolio assets, managing such a portfolio from a quantitative perspective.

RAM Risk analysis and mitigation (L5). To identify sources of flaws and other problems (e.g., requirements inconsistencies, poor portfolio management, lack of IS principles) in the IS architecture domain, and to take action to prevent such situations in the future.

SPD Standards and principles definition (L2). To define technology standards, policies and development principles stating direction or practice on how the collaboration should deal with the ISs.

\subsubsection{Process architecture}

BPD Baseline process architecture description (L2). To create a snapshot of the existing processes, identifying and analyzing what the current state of the $\mathrm{CNO}$ is concerning processes.

CAR Causal analysis and resolution (L5). To identify sources of flaws and other problems in the process architecture domain, and to take action to prevent such situations in the future.

EFC Event logs formal consistency (L4). To use event logs for checking traceability of execution processes during collaboration, and for controlling whether profitability estimates are realized. 


\subsection{THE ICONOS MM: THE FIRST VERSION}

IoPO Inter-organizational process optimization (L5). To evaluate the process architecture in order to deploy incremental and innovative improvements to gain cross-organizational efficiency and competitive advantage. A successful process optimization relies on effective process focus planning and process architecture definition.

OPP Organizational process performance (L4). To establish and maintain a quantitative understanding of the performance of the standard processes set in support of quality and process-performance objectives.

PAD Process architecture definition (L3). To establish and maintain a repository of CNO processes, assets and work environment standards. A successful process architecture definition depends on an effective baseline process architecture description.

PAF Process architecture target formulation (L3). To evaluate, select and design processes needed to support the desired To-Be state of the process architecture taking into account business and strategy drivers.

PFP Organizational process focus planning (L3). To plan, implement, and deploy process improvements based on a thorough understanding of strengths and weaknesses of the collaboration's processes and process assets. A successful process planning will be performed through effective process architecture definition.

PPM Process portfolio management (L3). To direct limited resources in terms of funds, people, etc., into the processes to create a holistic process orientation.

\subsubsection{Coordination}

coc Communication-oriented coordination (L3). To agree on communication channels, shared knowledge and learning in order to respond effectively to immediate client's needs and to determine what future markets will require.

DTS Direct supervision (L2). To supervise the work by specific persons who take the responsibility for the processes, providing instructions to others and monitoring their actions.

InCA Informal communication adjustment (L2). To adjust and control the work among the participating organizations by informal communication across them outside the imposed hierarchical constrains for day-to-day operations.

QRA Quantitative coordination analysis (L4). To use techniques (e.g., causal model analysis) to link the interrelationships, called coordination relations, to the local scheduling constraints of the participating organizations.

STD Standardization (L3). To coordinate work and interactions by standardizing the processes, outputs and/or skills among the participating organizations. 


\subsection{Literature survey}

Nine theories and eight models, developed elsewhere, were identified as potentially useful to give insights for understanding the processes related to B-ITa in CNOs presented in the previous section (e.g., [21, 22, 48, 57, 73, 81, 90, 95, 122, 136, $138,189,204,206,260,302,310,312])$. By 'model' we mean a conceptual model, i.e., a set of constructs used to describe B-ITa or a domain of B-ITa. By 'theory' we mean a model plus claims about empirical relations between some concepts, i.e., correlational or causal relationships.

Our position was that it would be practical for CNOs to have a selection of those processes in a single model. Figure 7.2 establishes a map relating these nine theories and eight models to the four B-ITa domains introduced in the previous chapter. These theories and models were identified in the semi-structured literature review that we conducted to select related work (see Chapter 4). That is, we used the same literature review effort to identify the theories and models mapped in Figure 7.2. However, the keywords used to identify these theories and models were related to the B-ITa domains, e.g., to identify work with IS architecture-related constructs we used: information systems architecture, information technology architecture, software landscape, applications blueprint. We make the explicit note that we also used keywords that we deemed connected with the definitions of the five levels that the ICoNOs MM covers. For example, to identify processes that could be included in LEVEL 4: Quantitatively Managed, we explicitly used the word quantitative together with the specific keywords we used for each B-ITa domains in our identification process.

It must be noted that each theory and model covers much more than the constructs (i.e., processes and process outcomes) we present in the figure. That is, in our research, we take from each theory/model only those constructs that could have a relation to the four B-ITa domains. Therefore, it can be argued that we did not include all theory/model constructs which may have a possible relation with the B-ITa domains. However, after an exhaustive analysis of the theories and models, we decided to include only general constructs. For example, two processes that can be found in the CMMI are 'Requirements development' and 'Requirement management' - see CMMI-DEV [57]. We did not include the 'Requirements development' process of CMMI in our mappings because this process covers specific characteristics that are considered, in a general way, by the 'Requirement management' process. Therefore, we did only take 'Requirement management' process into account in our mappings (see Figure 7.2). In this figure the acronyms PS, IS, PA and CO stand for partnering structure, IS architecture, process architecture, and coordination, respectively.

The leftmost and the rightmost columns in this figure present the theories or models taken from the literature. From each theory/model we have selected constructs, assigned these to a B-ITa process area and assigned the B-ITa process area to a B-ITa domain. For example, Gunderson's theory [122] of system safety analysis (depicted in the upper left-hand corner of Figure 7.2) is mapped onto the RAM B-ITa process area of the ICoNOs MM, where RAM stands for Risk Analysis 


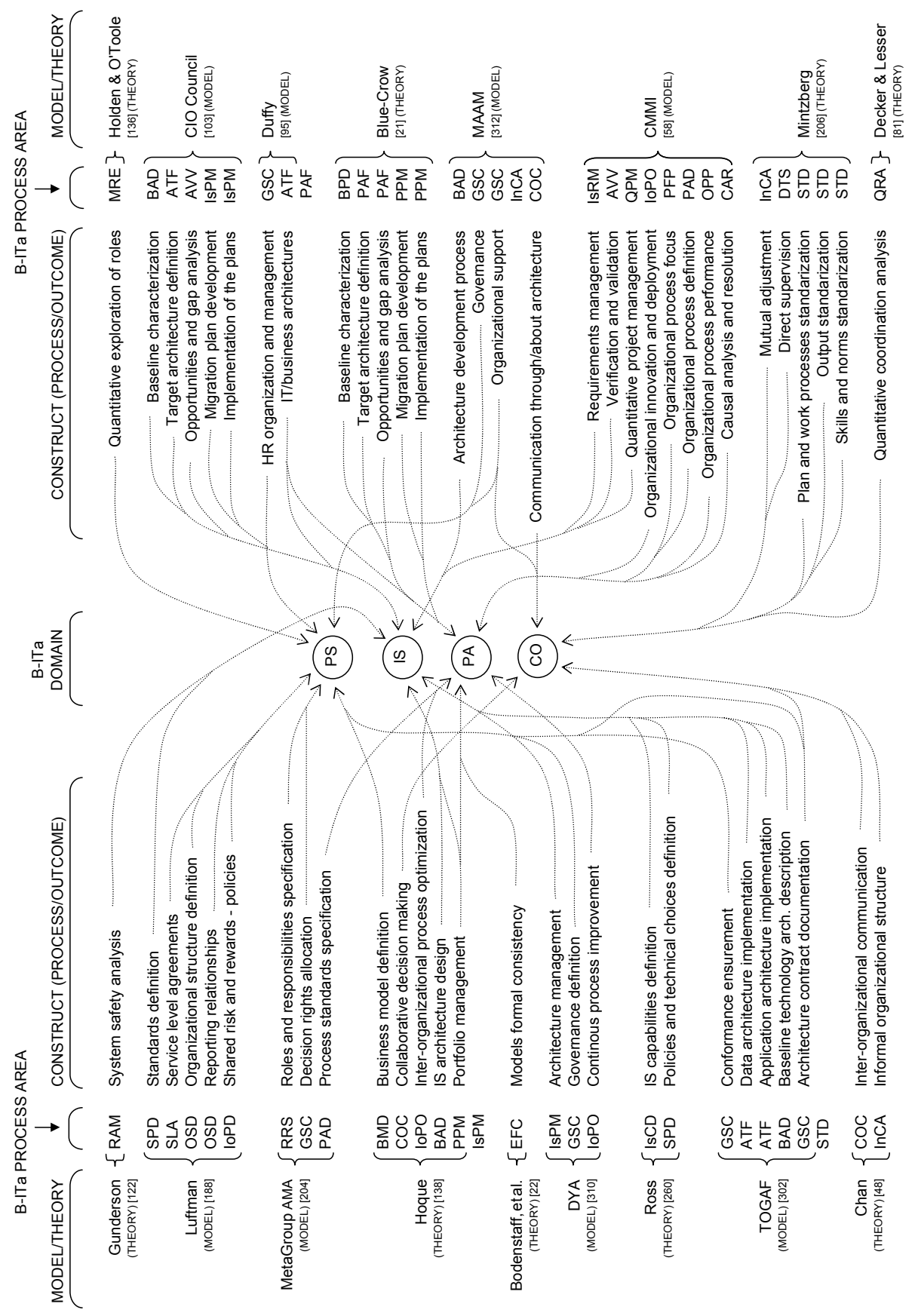

Figure 7.2: Map modeling theories applicable to the B-ITa domains. 
and Mitigation. The arrow connecting 'system safety analysis' to the oval labeled IS indicates that this is associated to the IS architecture domain. We make the note that the definitions of some constructs made us decide:

- to map them to more than one B-ITa process area. For example, Hoque's theory [138] of portfolio management can be applied to process architecture (PPM process area) and to IS architecture (IsPM process area).

- to merge constructs from the same theory/model, and/or from different theories/models, before mapping them to one B-ITa process area. For example, the constructs 'Data architecture implementation' and 'Application architecture implementation' of the TOGAF model [302] and the process 'Target architecture definition' of the CIO Council's model [103] and the construct 'IT/business architectures' of Duffy's model [95], relate to aspects that could be grouped in one process area of the ICoNOs MM that we called ATF, where ATF stands for IS Architecture Target Formulation.

In Section 7.4 we present a case study we conducted to identify whether the process areas of the ICoNOs MM were present in a real-life CNO, and to (implicitly) validate their positioning into the model. In the following, we first briefly present the theories and models applicable to the B-ITa domains (see Figure 7.2).

\subsubsection{Theories of B-ITa related constructs}

In this section, we present the nine theories we used for identifying B-ITa related constructs that helped us to define B-ITa process areas to include in our model.

\subsubsection{Gunderson's theory}

In his theory of system safety analysis, Gunderson [122] presents that system safety analysis involves elements of prevention, detection, containment and recovery in the IS architecture domain. System safety analysis improves quality and productivity in organizations by preventing problems related to the ISs. This theory is based in the premise that detecting problems after they have been introduced is not cost effective; it is more effective to prevent them from being introduced by using system safety analysis in the B-ITa projects. According to Gunderson, ISs can be affected both during design and operation not only by technical issues but also by organizational factors which can limit adequate IS risk analysis.

Since IS-related problems may have been previously encountered on other projects, system safety analysis activities can be considered as means for communicating lessons learned between projects assuring the continuously optimization of processes. This consideration led us to take Gunderson's theory as basis for the inclusion of Risk Analysis and Mitigation (RAM) as a process area at level 5 (optimized) in the IS architecture domain. 


\subsection{LITERATURE SURVEY}

\subsubsection{Hoque's theory}

In his efforts for answering the question: Why aren't we getting real business value out of technology? Hoque [138] presents the so called 'business technology management' theory. Specifically, his work introduces principles that support the alignment between business and IT. These principles cover issues related to the utilization of business modeling, the introduction of collaborating decisionmaking and the management of knowledge. Moreover, he also recognizes that "without concrete activities that you can perform to determine where you are today, to decide where you need to go, and to define how you should go about getting there, there's no guarantee that alignment will finally become a reality" [138, p. 77]. He therefore describes three activities of business technology management - business model definition, process optimization, and technology automation - and use a case study to show how these activities might work in a real-life business environment.

Because the explicit relation of the principles and activities included in Hoque's theory with the four B-ITa domains included in our ICoNOs MM, we considered them for inclusion in our mappings. We, specifically, took the main concepts they cover (i.e., business model definition, collaborative decision making, process optimization, IS architecture design, and portfolio management) to define B-ITa process areas for the four B-ITa domains.

\subsubsection{Bodenstaff et al.'s theory}

Logs help to monitor/manage ISs behavior/performance. Based on this statement, Bodenstaff et al. [22] claim that it is important to combine profitability models and process models with event logs. Event logs represent an additional view of the ISs. They contain data gathered from the running ISs of the partnering organizations in a CNO during the time they collaborate. The combination of the two models with event logs represents thus the currect ISs and they should therefore be consistent, i.e., not contradict each other. The theory and methods presented by Bodenstaff et al. [22] help to establish and maintain a quantitative understanding of the consistency between event logs and process and profitability models. This allows CNOs (i) to gain a clear view of the performance of their processes in support of quality and process-performance objectives, and (ii) to provide the data and models to quantitatively manage the CNOs projects because event logs enable traceability of execution processes during collaboration.

Therefore, this theory can be mapped to our B-ITa domains. Specifically, it was mapped onto the EFC process area of the process architecture domain where EFC stands for Event logs Formal Consistency, because its quantitative nature which is covered by the level 4 (quantitatively managed) in our model.

\subsubsection{Ross' theory}

Ross states that to achieve B-ITa, organizations must develop organizational competencies in IS architecture. A competency in IS architecture "is the ability 


\section{CHAPTER 7. B-ITA PROCESSES}

of a firm to create a mutually reinforcing pattern of evolving, tightly aligned business strategy and IT capabilities" $[260$, p. 2]. She identifies three steps for developing an IS architecture: (i) strategic objectives definition, (ii) key IT capabilities definition - for enabling the objectives defined in (i), and (iii) policies and technical choices definition for developing the IT capabilities. Several of the case studies Ross conducted for developing theory on IT capabilities illustrate that organizations improve their ability to define and achieve B-ITa by cumulating architecture-related experiences. "When used to enrich organizational learning, these experiences can create enterprise IT architecture competencies" [260, p. 3].

Ross also identifies particular stages that present an 'evolutionary path' of enterprise IS architecture competencies. This 'evolutionary path' idea on IS architecture competencies (which commonly is also seen in MMs) resulted in our motivation to consider Ross' theory as input for defining B-ITa process areas in the IS architecture domain. We took only the last two steps for developing an IS architecture because the first one is explicitly oriented to strategic issues which are out of the scope of our model - see Chapter 2.

\subsubsection{Chan's theory}

The main objective of Chan's work [48] was to identify and investigate components of B-ITa in real-life organizations and to distinguish among these components in terms of their importance for IT performance and competitive advantage. She found that the importance of alignment of formal structures is overstated. "An important alignment component that appears to require additional emphasis in future management practice is that of the informal organization structure" [48, p. 110]. Based on existing literature and eight case studies, Chan claims that the informal structure in an organization may be the most long-lasting aspect of B-ITa unlike the impermanent nature of the formal structure. Her findings reveal that while specific informal structures varied - i.e., they were not consistent or the same across organizations - informal structures and communication were repeatedly visible [48], and are thus essential for B-ITa improvements. This led us to use Chan's work as evidence for using the constructs inter-organizational communication and informal organizational structure when considering CommunicationOriented Coordination (COC) and Informal Communication Adjustment (InCA) as B-ITa process areas in the coordination domain.

\subsubsection{Holden and O'Toole's theory}

Holden and O'Toole [136] claim that communication is important to cross-organizational relationships. To test this claim, they examined if communication could in fact be a distinctive characteristic of this type of relationships. They state that the use of statistics and discriminant analysis can be useful to analyze crossorganizational relationships. Results from using quantitative techniques provide organizations with a precise assessment of roles and relationships indicating gaps between their As-Is and the To-Be perceptions. Implications from their study suggest that their 'relationship strength framework' can guide organizations in 


\subsection{LITERATURE SURVEY}

their allocation of those specific resources, behavioral and economic, which are required to structure cross-organizational relationships in an optimal way.

In their work, Holden and O'Toole [136, p. 547] conclude the ability of their framework "to delineate alternative governance structures and on the contribution of a communication belief component to the discrimination of varying relationship types". Their work was considered to define the MRE process area of the partnering structure domain, where MRE stands for Metric-based Roles Exploration.

\subsubsection{Blue-Crow's theory}

Blue-Crow is a consulting, training and supporting organization that provides business integration and enterprise architecture solutions (see http://www.bluecrow.com). Based on the premises that business processes need to be constantly reviewed and improved and that new business initiatives require the development of new processes, Blue-Crow developed a set of statements on modeling and analysis of business processes [21]. They state that in order to improve process efficiency and quality in an organization, the processes need to be documented and analyzed on the basis of both the current (As-Is) and the future (To-Be) processes of the organization. The key goal is to identify improvement opportunities that eventually must be planned and implemented according to the requirements of the organization itself.

Blue-Crow's theory matches the main constructs considered in the CIO Council's model [103] presented in Chapter 4, i.e. baseline characterization, target architecture definition, opportunities and gap analysis, migration plan development, and implementation of the plans. This led us to include Blue-Crow's theory in our mappings. However, instead of being focused on IS architecture as the CIO Council's model does, Blue-Crow focuses on process architecture.

\subsubsection{Mintzberg's theory}

According to Mintzberg, "every organized human activity... gives rise to two fundamental and opposing requirements: the division of labor into various tasks to be performed and the coordination of those tasks to accomplish the activity" [206, p. 2]. Structure in an organization is simply the way in which the organization divides productive work into distinct tasks and achieves coordination of these tasks. Mintzberg's theory states that organizations have only a few basic structures. These are identified by how key organizational attributes - such as organizations' component parts, the mechanisms they use to coordinate their work, the elements of their organizational design, their power systems, and their external environment - interrelate as parts of the organization as a whole [206]. Furthermore, he explains coordination mechanisms to explain the fundamental ways in which an organization coordinates its work.

Because the explicit relation of Mintzberg's theory with the coordination domain included in our ICoNOs MM, we considered the five coordination mechanisms 


\section{CHAPTER 7. B-ITA PROCESSES}

mentioned in his theory (i.e., mutual adjustment, direct supervision, standardization of work processes, standardization of outputs, and standardization of skills) to define three B-ITa process areas, namely Direct Supervision (DTS), Informal Communication Adjustment (InCA) and Standardization (STD).

\subsubsection{Decker and Lesser's theory}

Decker and Lesser [81] believe that analyzing coordination in a collaborative environment requires an understanding of the interaction between the collaborating organizations, their problem, and their environment. They outline a methodological approach toward building a theory of coordination. Decker and Lesser infer that the complexity of the interrelationships across organizations makes coordination difficult. They describe, from a quantitative perspective, a causal model that links the interrelationships among the collaborating organizations to the local scheduling constraints of each of the organizations. However, they state that it is not only the presence - or absence - of a coordination relationship that is important, but its quantitative properties (e.g., how likely it appears or how significant its effect is). These properties determine the utility of a specific coordination relationship in the context defined by an environment and a problem to be solved. Such ideas were the reason to include this theory in our mappings. We found Decker and Lesser's theory suitable to define the QRA process area at level 4 (quantitatively managed) of our ICoNOs MM - where QRA stands for Quantitative coordination Relation Analysis.

\subsubsection{Models covering B-ITa related constructs}

Five of the models (Luftman's model [189], CIO Council's model [103], Duffy's model [95], van der Raadt et al.'s MAAM [312], CMMI [58]) included in Figure 7.2 have been already introduced in Chapters 2 and 4 . In the following, we present the three remaining models, which we found useful for the purpose of identifying B-ITa related constructs.

\subsubsection{MetaGroup's model}

MetaGroup, a global IT consulting and market research firm, developed an architecture maturity audit [204] in order to help identifying an organization's context (history, culture, processes, people and technology) in a disciplined way. MetaGroup believes that developing a through understanding of an organization supports the value of the enterprise architecture (EA) of such an organization. This understanding must be focused on the context of the organization itself because its organizational context "provides valuable clues about when, where, and how to advance an EA program. A common approach of many architecture efforts is to identify that context and work within its constraints" [204, p. 1]. After assessing the architecture maturity of an organization examining several prerequisites included in the model of MetaGroup, organizations can apply 'corrective' mechanisms to make the organizational context favorable for EA improvements. 


\subsection{LITERATURE SURVEY}

Because this architecture maturity audit examines the context where EA improvements can be successful, this MetaGroup's model explicitly covers constructs related to B-ITa. Specifically, it covers constructs related to the partnering structure and the process architecture domains (as presented in Figure 7.2) that helped us to define the process areas Roles and Responsibilities Specification (RRS), Governance Structure and Compliance (GSC) - both process areas included in level 3 of the partnering structure domain - and Process Architecture Definition (PAD) - included in level 3 of the process architecture domain.

\subsubsection{DYA}

DYA (DYnamic Architecture) was developed by Sogeti (http://www.sogeti.nl/) and has become a de facto standard for working with architecture in Dutch organizations. It concerns the creation and management of an EA, which has to be dynamic in nature. DYA was developed based on experiences in the field regarding the manner in which architecture was handled in real-life organizations. One of the instruments covered by DYA is an architecture maturity matrix [310]. This matrix helps to assess the state of the architectural function in an organization. It can be used to ensure that the right aspect is given the right amount of attention at the right time ('just in time, just enough' approach). The maturity matrix supports the identification of improvement issues on 18 areas, among which we can list: involvement of development process, roles and responsibilities, quality management, commitment and motivation, controlling, maintenance of deliverables, etc. Since DYA is focused on EA issues, it was useful to define B-ITa process areas for the IS architecture and process architecture domains.

\subsubsection{TOGAF}

TOGAF, The Open Group Architecture Framework [302], is an industry standard architecture framework - a detailed architectural development method and a set of supporting tools - that may be used freely by any organization wishing to develop an EA for use within that organization (http://www.opengroup.org/togaf/). "TOGAF distinguishes four kinds of architectures, namely business architecture, data architecture, application architecture and technology architecture, where data architecture and application architecture is sometimes referred to as IS architecture" [351, p. 265]. Specifically, it (i) provides a high-level introduction to some of the key concepts behind EA, (ii) describes a step-by-step approach to developing an EA, (iii) introduces a virtual repository of EA assets, and (iv) comprises a set of tools and techniques available for use in applying TOGAF.

We identified a variety of B-ITa related processes to consider in our mappings. For example, conformance ensurement, data/application architecture implementation, baseline technology description, and architecture contract documentation. These processes helped us to define B-ITa process areas for the four B-ITa domains included in the ICoNOs MM (see Figure 7.2). 


\subsection{Case study 5: Regional government network}

After introducing the first set of B-ITa process areas included in the ICoNOs MM, and presenting the literature survey that helped us to identify these process areas, in this section we describe the case study we conducted to confirm our choice of the B-ITa process areas in a real-life CNO.

The site investigated in our fifth case study was the same CNO we studied in the third case study presented in Chapter 6, i.e., the Overijssel CNO. We decided to use the same organization since we wanted to gain a more complete picture of their B-ITa project and because they were willing to grant us access. This case study was conducted after conducting the third case study presented in Chapter 6 where the objective of the study was different. The objective of this case study was to identify whether the process areas identified in the literature survey, and already included in the first version of the ICoNOs MM (see Figure 7.1) could be observed in the Overijssel CNO. We claim that the B-ITa process areas of the ICoNOs MM are the required processes to implement for making improvements in the B-ITa domains. If the process areas prove to be important in the results of our case, then we have an empirical ground for their inclusion in our MM. We therefore wanted to identify the occurrence of the B-ITa process areas in the Overijssel CNO (see Section 7.4.1 for further explanation). This case study helped us to come out with a list of B-ITa process areas that were PERFORMED or NOT PERFORMED in a real-life CNO (see Section 7.4.2). The research questions (composed of a descriptive and an explanatory question) addressed with this case were:

1 What are the B-ITa process areas performed by a CNO for improving its $B-I T a$ ? Why?

2 What are the B-ITa process areas not performed by a CNO to improve its $B$-ITa? Why?

These two questions led to the formulation of the next hypothesis:

If a CNO strives to improve B-ITa, then it is possible to identify, in such a CNO, the process areas included in the ICoNOs MM (see Figure 7.1).

Furthermore, considering the five levels that our MM has (see Section 4.2.2 in Chapter 4), it would be possible to identify:

- at level 2, process areas that are planned and executed in accordance with policy; employ skilled people who have adequate resources to produce controlled outputs; are monitored, controlled, and reviewed, but not from the entire CNO perspective.

- at level 3, process areas that focus on making improvements in the standardization and management of B-ITa domains. Processes that are performed 


\subsection{CASE STUDY 5: REGIONAL GOVERNMENT NETWORK}

from a CNO perspective, and that are well characterized and understood, and are described in standards.

- at level 4 , process areas that use statistical and other quantitative techniques, where quantitative objectives for quality and process performance are established and used as criteria in managing the processes themselves.

\subsubsection{Data collection and analysis process}

The case study research design involved multiple sources of data, which suggests that construct validity must be addressed, and that we could generalize results to valid statements [348]. The variety of data sources were: (i) professionals from the case study, (ii) meetings, (iii) documents related to WABO, LVO, the WABOICT project ${ }^{1}$, and past projects, and (iv) illustrative materials (e.g. newsletters and videos). This combination of sources permitted triangulation of the data to increase the strength of the findings. Furthermore, limiting the study to one CNO in one sector (i.e., the public one) facilitated the analysis of all these sources of evidence. However, as we have already mentioned in Chapter 3, the case site used was not selected randomly. Therefore, it can be claimed that it is not possible to generalize findings to a wider population of organizations, as it is not a randomly selected sample of this population. Nevertheless, as we can generalize to theory (see Section 7.4.4), part of the results can be drawn on by others who work in an environment where B-ITa plays a valuable role.

The data collection techniques used with the professionals and the meetings were semi-structured interviews ${ }^{2}$ and observation, respectively. The interviews were conducted with 8 persons on a one-to-one basis. As in the case study 2 presented in Chapter 6, we mitigate impression management and retrospective sensemaking bias by interviewing different and highly knowledgeable professionals who view the Overijssel CNO from diverse perspectives. The professionals included people from the different collaborating organizations and from diverse functional areas. Table 7.1 classifies the interviewees based on their expertise (B-ITa domain) and the organization they are affiliated with.

The duration of each interview was approximately 1 hour. The interviews were taped. This was done with the consent of each interviewee. When interviewing, we were primarily interested in identifying whether a process area was or was not performed in the Overijssel CNO (see the research questions presented above which were addressed with this case study). The interviews were theory-driven interviews [228]. We started each interview by presenting the overall definitional foundations and context of our research to each interviewee. The goal was to make the interviewee understand our research context and concepts (B-ITa domains and process areas in CNO settings). With such an understanding, the interviewees had the opportunity to explain and clarify their own thinking.

\footnotetext{
${ }^{1}$ Explanation of these acronyms can be found in Chapter 6 .

${ }^{2}$ The questionnaire used in the interviews can be found in Appendix C (in Dutch).
} 


\section{CHAPTER 7. B-ITA PROCESSES}

In this context, the interviewee provided more meaningful answers to our questions, leading to new questions that were useful to clarify our own ideas. For example, when looking for evidence supporting the performance of the Roles and Responsibilities Specification - RRS - process area, we started asking: Was the definition of the partnering structure necessary to efficiently collaborate? Because interviewees were encouraged to describe situations in detail, we got as answer explanations concerning the organizational structure of the Overijssel CNO and the relationships among the participating organizations at functional level (see Figure 6.6). As a result, specific follow up questions - as part of the hermeneutic circle $^{3}$, were: Which roles were required? How did you define an accountability framework? Who was involved? Was this specification of roles and responsibilities necessary? The interviewee also presented to us several documents when we could clearly identify partnering structure process areas, and specifically, figures and narrative concerning the RRS process area - see [178, 244].

Table 7.1: Distribution of interviewees' expertise.

\begin{tabular}{l|c|c|c|c}
\hline Field of expertise/B-ITa domain & A & B & C & Total \\
\hline \hline Partnering structure and coordination & 1 & & & 1 \\
IS architecture & 2 & & 2 & 4 \\
Process architecture & 2 & 1 & & 3 \\
Total & & & & \\
\hline
\end{tabular}

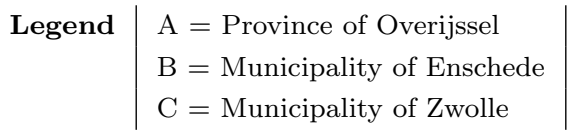

If the interviewees answered positively providing explanations of the process itself, participating in meaning making, and presenting evidence from documents, then we considered that the process area in concern was performed in the Overijssel CNO (and we put the checkmark $\checkmark$ in the PERFORMED column of Figure 7.4; otherwise we filled the NOT PERFORMED column). However, if a B-ITa process area was not performed in the $\mathrm{CNO}$, the presence of the results of performing such a process area was enough to consider the process area was carried out. That is, if we did not get explanations from the interviewees about the process in concern itself but only evidence from documents and/or indications of specific activities which directly, or indirectly, brought the results that are expected in the B-ITa process area (see the definitions of the process areas in Section 7.2.1), then we also considered such a process area was performed. Within the ICoNOs MM context, although the B-ITa process areas are required processes to implement for making improvements in the B-ITa domains, one can implement alternative practices in substitution for the practices that are included in each of the process

${ }^{3}$ The hermeneutic circle [165] suggests that proper understanding is achieved through iterations in a dialogical reflection $[160,306]$. 


\subsection{CASE STUDY 5: REGIONAL GOVERNMENT NETWORK}

areas of the ICoNOs MM (see Chapter 8). The only condition is that the goals of each process area must be satisfied in order to reach a specific maturity level. In the Overijssel CNO, (i) performing a formal process to identify the software application requirements (IsRM process area) was not the only reason to know about the required ISs and (ii) the inter-organizational policies definition (IoPD process area) has not influence on the high commitment and mutual benefits perception they have. For example, the Overijssel CNO is regulated by the plans and laws dictated by VROM, and uses external advices and customer satisfaction research results to identify its ISs requirements.

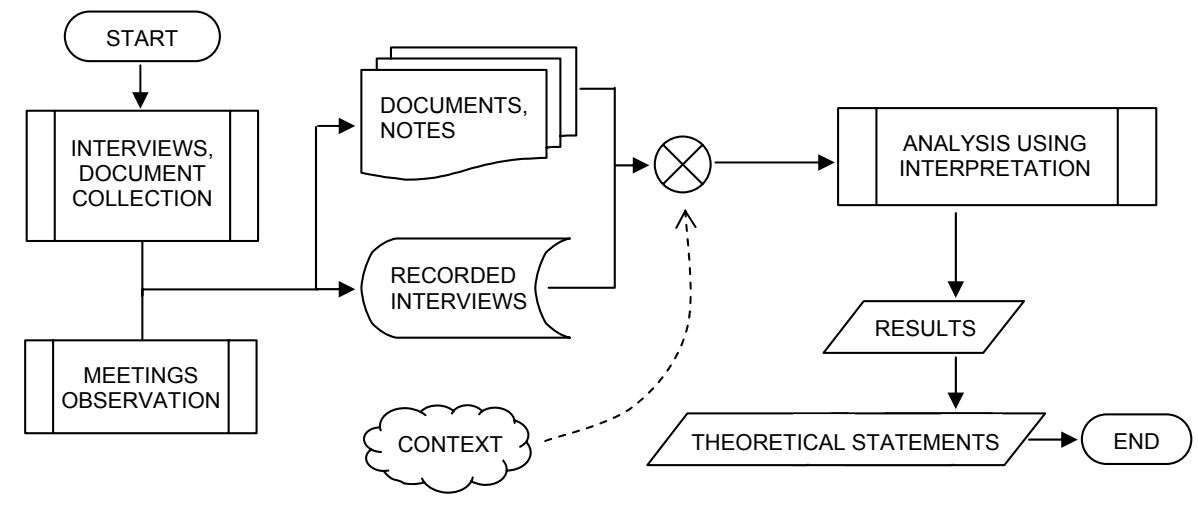

Figure 7.3: The data analysis process.

Figure 7.3 illustrates the analysis process we followed. The data analysis was conducted using interpretation from an hermeneutic perspective (see Chapter 3). We used hermeneutics in the interpretation process because we had multiple data sources. In this case, hermeneutics helped to obtain results from analyzing the taped interviews, the documentation, the illustrative materials, the meeting observations, and the CNO altogether.

In the next three sections we present the case study findings (Section 7.4.2), several final statements derived from the analysis of evidence (Section 7.4.3), and the vality threats we addressed (Section 7.4.4).

\subsubsection{Case study findings}

Figure 7.4 presents an overview of the process areas found in the Overijssel CNO. In the next paragraphs, we present the general findings in each B-ITa domain, and their respective list of B-ITa process areas following the next structure: for each process area, we first introduce its abbreviation and name ${ }^{4}$. Then, we state if the process area was or was not performed in the Overijssel CNO, and we present the analysis of the findings related to such a process area. In this section, we list the process areas in order of level 2 up to level 5 starting with the performed ones.

${ }^{4}$ Its definition can be found in Section 7.2.1. 


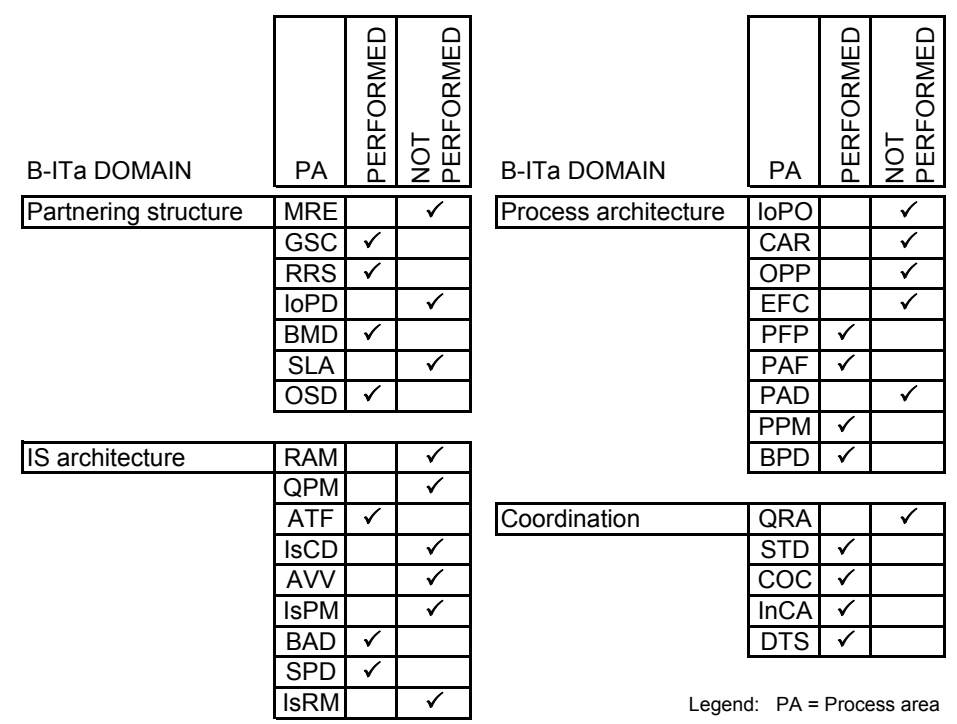

Figure 7.4: B-ITa process areas performed in the Overijssel CNO.

\subsubsection{Partnering structure}

In the interviews, some of the first documents that the interviewees presented to us were documents with information related to the relations between the participants and what was expected from each of them. These documents are the following:

- Starting architecture for the national online all-in-one service for the environmental permit - in Dutch: Startarchitecture landelijke voorziening omgevingsvergunning [323],

- Looking to the future! - in Dutch: Met het oog op morgen! [245], and

- National online all-in-one service for environmental permits (Set up v 1.1) - in Dutch: LVO Inrichting v 1.1 [139].

From them, we could practically observe the future situation of the Overijssel CNO (see Figure 7.5) where the roles of the participating organizations and the functional level of the future ISs were depicted.

In each of the interviews, we asked whether the interviewee was aware of the benefits his/her organization would obtain from collaborating in the Overijssel CNO. All interviewees came with a similar answer: "yes, our main benefit is to enhance our image as public entity that contributes in a national project to improve the performance of the public sector in the Netherlands; this compelled us to strongly commit to collaborate for improving the environmental permit process". No interviewee mentioned that such a commitment came from the definition of 


\subsection{CASE STUDY 5: REGIONAL GOVERNMENT NETWORK}

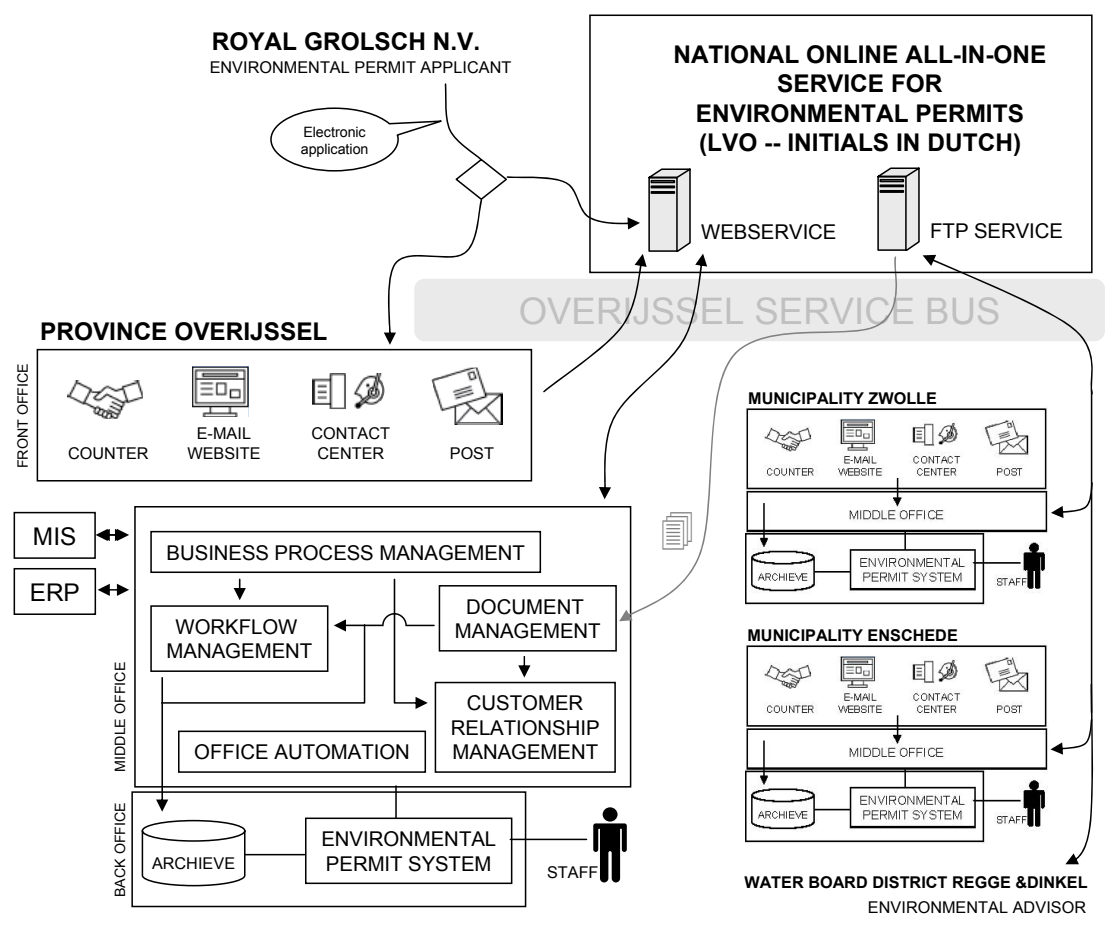

Figure 7.5: The Overijssel CNO: Future situation (adapted from [139, 245, 323]).

shared risks or policies (as it commonly happens in a CNO). However, one of the interviewees explicitly mentioned that "this commitment decreases the formal definition of service level agreements among us; there is a team that is starting with this definition; personally, I believe it is important to agree on service levels in order to work efficiently and to reduce collaboration problems".

BMD Business model definition.

PERFORMED

It was clear that the definition of a blueprint of the CNO was the first activity they did to begin to collaborate. The Overijssel CNO described the relation of the different participating organizations in order to know what was expected from each of them and to present how they fitted together.

OSD Organizational structure definition.

PERFORMED

The alignment of business and IT was also facilitated by the organizational structure that complements its goals. The understanding that goals and structure need to be linked was well-embedded in the organizational structure of the Overijssel CNO. 


\section{CHAPTER 7. B-ITA PROCESSES}

GSC Governance structure and compliance. PERFORMED

The interviewee pointed that "increasing emphasis was being given to the balance of responsibilities". The major responsibilities in the WABO-ICT project were in the development of the technological architecture, the oversight of the IS, and the description of the collaborative processes and interfaces. The match between organizational structure and responsibilities was also illustrated in (i) the functional level of the IS, as we could see in some documents (e.g., see Figure 7.5), and (ii) the way they organized and related future activities taking in consideration resources and roles. For example, in one of the meetings we observed that for planning the first test pilot of the IS in the physic location of each participant, they listed the required resources (human and technological) in order to come up with an efficient plan.

RRS Roles and responsibilities specification.

PERFORMED

The same interviewee explained to us that since the project began, "there was a clear indication of who was responsible for achieving specific goals, and what the role of each participating organization was". In general, the Overijssel CNO, as a networked organization in the public sector, has a (i) well-defined hierarchy of authority with powers and responsibilities understood by all and (ii) a clear-cut division of work among the participants and people.

IoPD Inter-organizational policies definition.

\section{NOT PERFORMED}

The interviews revealed that the commitment and mutual benefits perception at each participant was high. However, the inter-organizational policies definition has not influence on this situation. Their high commitment came from a specific individual goal of each participant: to be present and to contribute in a project (i.e., the WABO-ICT) that will have national impact in the future.

SLA Service level agreements definition.

\section{NOT PERFORMED}

Service level agreements were still not defined in the Overijssel CNO. Experience in the definition of SLAs was not a problem. They know exactly what to do, how to do it, and what they need to get success in the description of agreements on the deliverables, quality, and fitness-for-purpose of services. Although the project is already testing the IS, they are in the very initial stage to define SLAs. It seems that the way of work and the formal commitment of the participating organizations reduced the necessity of agreements description in the beginning of the project. 


\subsection{CASE STUDY 5: REGIONAL GOVERNMENT NETWORK}

MRE Metric-based exploration of roles. NOT PERFORMED

No quantitative technique was used to explore and/or analyze the roles in the Overijssel CNO.

\subsubsection{IS architecture}

In this study, the 4 professionals that we interviewed in the IS architecture domain indicated they believe that the CNO is highly dependent on ISs. We had access to different documents presenting the principles and norms they use for the development of the IS architecture (e.g., NORA [161], SOA [281], ISO 17799:2005 [147]). The Dutch Government Reference Architecture (NORA - initials in Dutch) is a set of models and principles showing how e-government works. They present the way in which it is possible to collaborate, to link processes smoothly to one another and to exchange data. One of the interviewees explained us the deontic nature of such models and norms stating that "NORA does not say anything about the content of the government service provision. For example, you can make an analogy with the road traffic infrastructure. Everyone expects that this infrastructure enables effective and efficient transport. An implicit assumption in this situation is that there is a road system of national, regional and local roads and there are traffic rules (for example we all drive on one side of the road). This is a typical assumption that would be included in NORA as a principle. Where exactly the roads are and in which side of the road we drive are decisions that the governments have made among themselves. If we apply this to NORA, we can see that NORA contains guidelines for (i) the exchange of data on a national scale and (ii) the definitions of data reports. However, NORA does not contain any elaboration of the infrastructure or the contents of the reports that public organizations exchange". In the Overijssel CNO, each of the three participating public organizations (i.e., the province of Overijssel and the municipalities of Zwolle and Enschede) also has its own set of principles based on NORA. Such principles, together with the NORA, are the basis for the definition of specific principles and explicit requirements in the WABO-ICT project.

Furthermore, the interviewees presented us documents presenting the results of work sessions they had to:

- create an overview of the ISs that each participant has for the extension of environmental permits (e.g. Figure 7.6 presents part of such an overview in the province of Overijssel $\left.{ }^{5}\right)$,

- define which ISs help what processes in the strategic, tactical and operational levels (see Figure 7.7), and

- list the ISs required for the collaborative work (see Figure 7.8).

${ }^{5}$ The figures presented as examples of evidence in the rest of this chapter have been intentionally blurred for anonymity of the data. 


\section{CHAPTER 7. B-ITA PROCESSES}
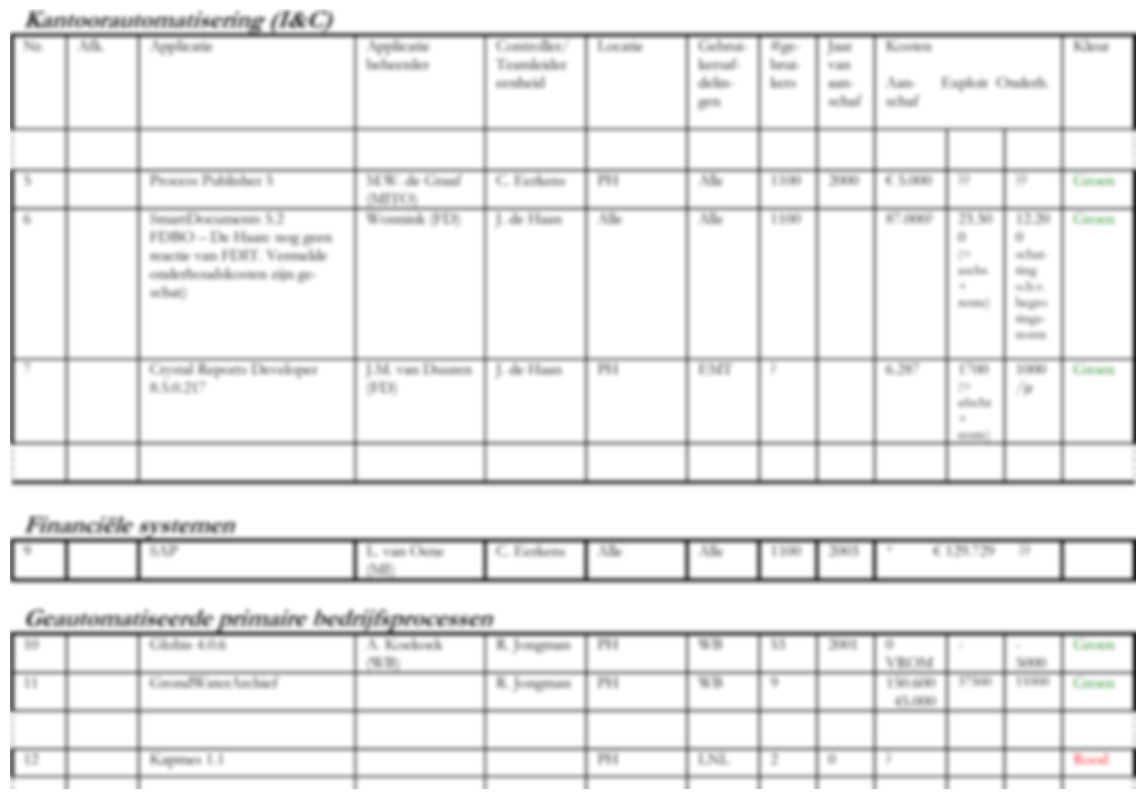

Figure 7.6: Overview of the ISs As-Is state in the province of Overijssel.

SPD Standards and principles definition.

PERFORMED

As explained above, the Overijssel CNO bases its work on several IS architecture standards and principles. Those standards and principles were applied to the project to guarantee the development of high-quality service for environmental permits.

BAD Baseline IS architecture description.

ATF IS architecture target formulation.

PERFORMED

When the project started, they created a snapshot of the existing ISs in order to have a clear view of the current situation of the CNO concerning ISs they had. The Overijssel CNO followed the same process to define its IS To-Be state.

IsRM IS requirements management.

AVV IS architecture verification and validation.

\section{NOT PERFORMED}

Although they were aware of the required ISs, they did not conducted (i) a formal identification of the IS requirements, and (ii) a gap analysis to know the suitability of the ISs already in place. None of the interviewees 


\subsection{CASE STUDY 5: REGIONAL GOVERNMENT NETWORK}

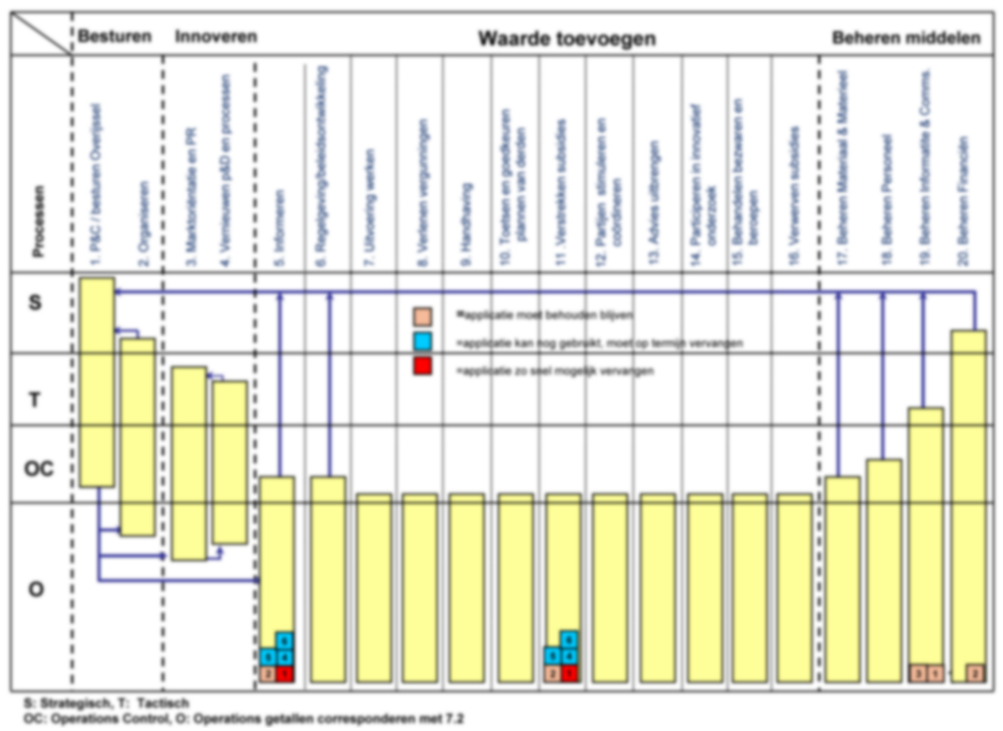

Figure 7.7: General processes supported by ISs in the Overijssel CNO.

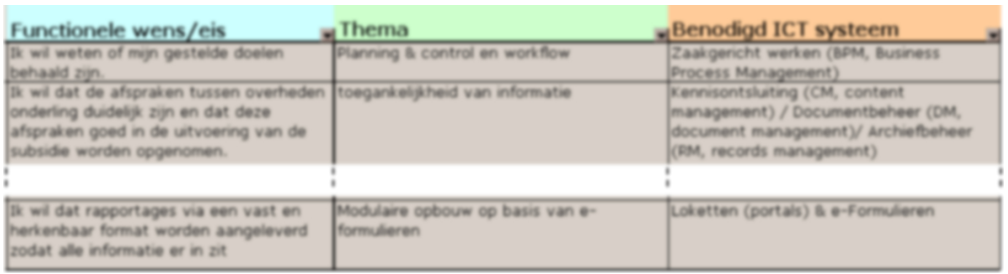

Figure 7.8: The ISs To-Be state in the Overijssel CNO: An example.

mentioned that these two activities were formal processes performed in the Overijssel CNO, even though they were aware of the importance of the results of such activities to support its B-ITa.

IsCD IS capabilities definition.

IsPM IS portfolio management.

QPM Quantitative IS portfolio management.

NOT PERFORMED

An identification of the $\mathrm{CNO}$ ability to achieve new forms of competitive advantage by ISs was not present. We believe that this occurs since the B-ITa driver of the Overijssel CNO is to improve quality and to increase effectiveness instead of innovation and risk management. Such situation also affects to manage the IS portfolio effectively. The Overijssel CNO has not 


\section{CHAPTER 7. B-ITA PROCESSES}

strategic innovation planning and it does not use risk management techniques. As a result, the selection of ISs in accordance with resources, needs and changing situations is inadequate to minimize risks. One interviewee pointed that IS portfolio management within their particular organization was partially performed. "Our organization tries to make fact-based, datadriven decisions using a consistent and disciplined approach to analyze information in a way that helps to optimally allocate scarce resources to ISs projects". However, although one participating organization take actions related to IS portfolio management, it did not mean that the CNO does the same.

RAM Risk analysis and mitigation.

NOT PERFORMED

No system safety analysis activities were performed to assure continuous optimization of the IS architecture in the Overijssel CNO.

\subsubsection{Process architecture}

When starting collaborating the participating organizations described the As-Is state of their process architecture. Later, one of their main activities was to define the To-Be state of such architecture. They spent considerable time and effort in working sessions to design the choreography of all (individual and collaborative) processes needed to reach the goals of the CNO (see Figure 7.9).

The author of this dissertation was present in some of the sessions, and personally witnessed how they took project portfolio management practices into account during the process design process. For example, the allocation of human and technological resources was implicit in the process architecture design process. The results of the working sessions included (i) a workflow process that encompasses project approvals, checkpoint reviews, and periodic status reporting at project and statewide levels, (ii) a plan to ensure that the work is done acceptably and that the project is in position to complete its phases successfully, and (iii) documents that follow recognized best practices for project management (e.g., PMI practices as message/document-driven controls, scope statement definition and work breakdown structure).

BPD Baseline process architecture description.

PAF Process architecture target formulation.

PERFORMED

As in the IS architecture domain, a blueprint of the current and To-Be state of the collaborative processes was easily found in some of the studied documents.

PPM Process portfolio management.

PFP Organizational process focus planning. 


\subsection{CASE STUDY 5: REGIONAL GOVERNMENT NETWORK}

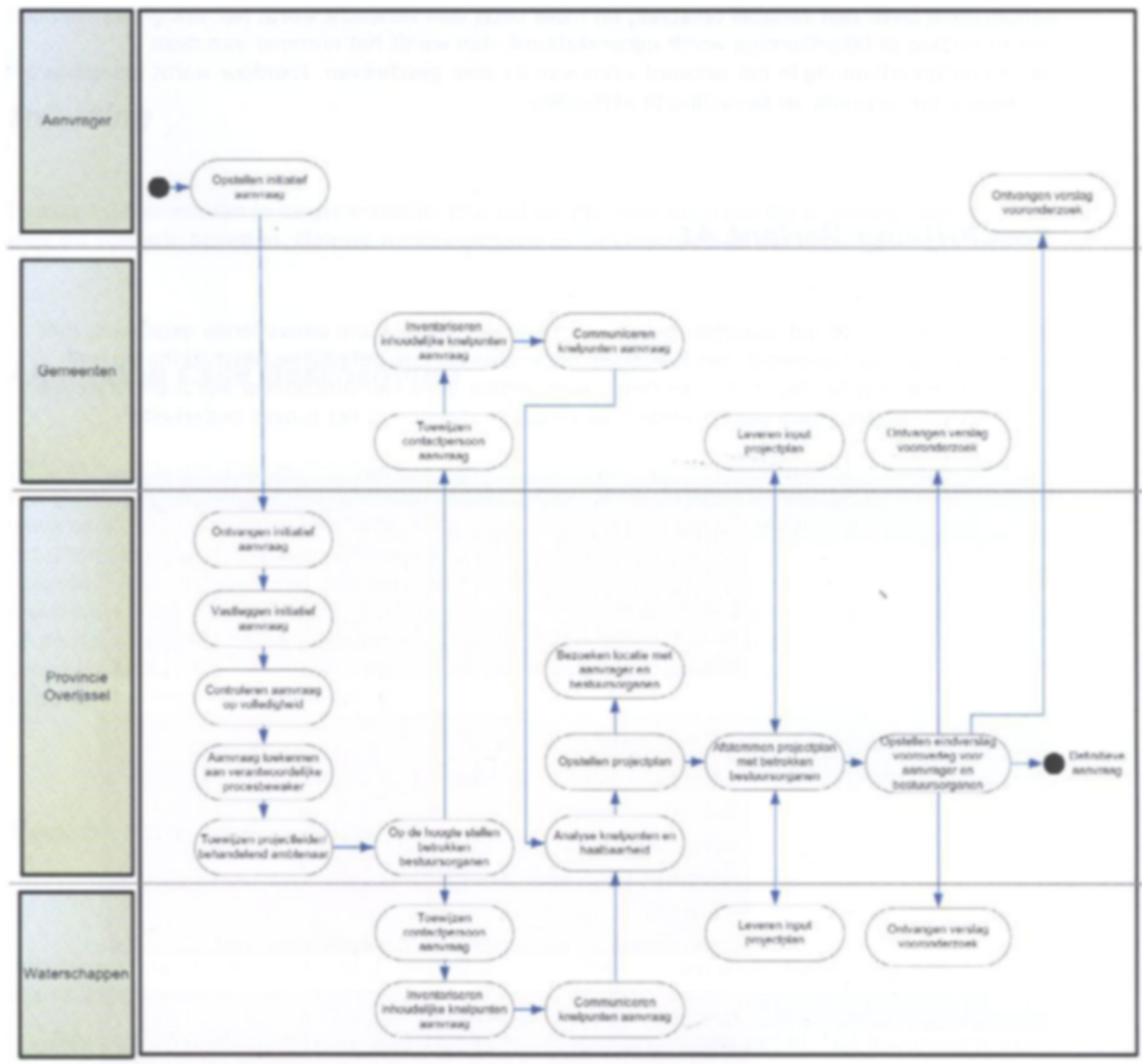

Figure 7.9: To-Be state of one collaborative process in the Overijssel CNO.

\section{PERFORMED}

Documents, and specially our observations in some of their work sessions for designing collaborative processes, gave us evidence to identify the presence of process portfolio management issues. A session always took in consideration the results of previous sessions. Therefore, they could plan and implement improvements based on an understanding of the pros and contras from past work. "The discussions on process improvement and optimization always begins by describing what goes into the process model and what we learn from previous sessions".

PAD Process architecture definition.

EFC Event logs formal consistency.

\section{NOT PERFORMED}

An interesting insight was to find again evidence to support a result from the IS architecture domain: best practices applied in one of the partici- 


\section{CHAPTER 7. B-ITA PROCESSES}

pating organizations are not automatically applied in the entire CNO. For example, a control mentality pervades from top to bottom in the province of Overijssel. This 'obsession' with control attempts to eliminate uncertainty so that the organization performs its tasks smoothly and without problems. In the province of Overijssel, quality assurance is an important control-related domain considered for its B-ITa efforts. Quality assurance in this participating organization implies (i) to maintain a repository of the processes and assets in order to check their compliance with national laws, quality standards (e.g., ISO 9001:2000), and the requirements (PAD process area); and (ii) to use logs of the process planning IS to identify flaws in process execution in order to take corrective and preventive measures (EFC process area). However, such processes are still not implemented for the collaborative processes of the entire Overijssel CNO.

OPP Organizational process performance.

\section{NOT PERFORMED}

No quantitative technique was used to understand/analyze the performance of the processes set in support of quality and process-performance objectives.

CAR Causal analysis and resolution.

IoPO Inter-organizational process optimization.

\section{NOT PERFORMED}

We did not identify processes that were oriented to continuously optimize the range of process performance through both incremental and/or innovative improvements.

\subsubsection{Coordination}

When we asked about the way they coordinate their work, our interviewee responded: "there is a consultant hired from an external company that coordinates the work in this project; several other people are also responsible for coordinating and supervising activities in each of the domains included in the ICoNOs MM". When we were interviewing the person with expertise in coordination, he also stressed how political issues affect the informal communication among the participating organizations. "Sometimes, each organization becomes jealous of its own work and exclusive privileges to do something. Therefore, it finds ways to protect itself against the pressure of the network or oversteps. This restricts a proper communication among the organizations and its members, and it drives a kind of competition". However, "the coordination team tries to minimize this undesirable situation by organizing social events based on informal settings to encourage the synergy and the raising of new ideas for working". They have managed to create an organization more concerned in the service to be provided than in the political games that could arise. 


\subsection{CASE STUDY 5: REGIONAL GOVERNMENT NETWORK}

DTS Direct supervision.

InCA Informal communication adjustment. PERFORMED

Although standardization (see below) helps to coordinate the interdependencies among the participating organizations in the Overijssel CNO, differences in meanings remain creating ambiguities which leads to have conflicts. As presented above, the coordination team tries to avoid these conflicts by encouraging the use of informal communication among the participants. However, they have found that conflicts cannot easily be handled by informal communication only since this kind of communication is sometimes held back by the standardization itself. Thus, they restrict the raise of conflicts by direct supervision. The Overijssel CNO has persons who take the responsibility for the processes and provide instructions to others to monitor their work.

STD Standardization.

PERFORMED

The Overijssel CNO tries to have high rationalized activities resulting in simple and repetitive tasks. This situation leads to have a sharp division of work and to depend primarily on standardization of its processes and skills for coordination.

coc Communication-oriented coordination.

PERFORMED

In addition to the use of informal communication to encourage synergy in the Overijssel CNO and to coordinate the work, coordination is usually achieved with some kind of 'formal' communication or information exchange. We identified through one interview and some meeting observations that in the Overijssel CNO, agreements on communication take place. These agreements are on either horizontal (or crosswise) communication with the help of informal communication adjustment, or on vertical communication in the form of standardization or direct supervision. Bringing members of the participating organizations to the required level of understanding to get their job done was a practice we identified within the WABO-ICT project. In addition, this CNO uses what each of the participants learns in interaction with the citizens who apply for the environmental permit both to respond to immediate needs and to determine future citizens requirements. For example, "each participating organization has its own knowledge in-house, i.e., each organization has different professionals that are experts in each of the permits (i.e., residency, spatial planning, environment, etc.) gathered within the environmental permit. They need to communicate with each other in order to share knowledge and to learn from each other" to produce, for instance, a complete process architecture of the To-Be state. 
CHAPTER 7. B-ITA PROCESSES

QRA Quantitative coordination relation analysis. NOT PERFORMED

No quantitative technique was used to explore and/or analyze coordination mechanisms in the Overijssel CNO.

\subsubsection{Final statements}

In the next paragraphs, we present (i) two general conclusions (i.e., findings no specific for a particular B-ITa process area) that are important when working for achieving B-ITa in a CNO, and (ii) eight B-ITa best practices derived from our results.

\subsubsection{General conclusions}

1. The study findings reveal that even though a process area is considered to achieve B-ITa in one participating organization, member of a $\mathrm{CNO}$, such a process area is not automatically taken into account to achieve B-ITa at CNO level. For example, in the CNO studied, we identified that the province of Overijssel does perform the SLA process area of partnering structure, the PAD and EFC process areas of process architecture, and the IsPM process area of IS architecture. However, these process areas are not performed in the CNO. The province of Overijssel as a single organization could achieve higher B-ITa maturity than the Overijssel CNO as a whole (for understanding of this difference, please refer to Section 1.2.1 of Chapter 1 where we explain the scope of the ICoNOs MM).

2. We have also evidence to conclude that there is a relationship between status enhancement and coordination mechanisms, and the level of commitment of the operational stakeholders towards the B-ITa project success. In a $\mathrm{CNO}$, the most transparent way of cooperation is an explicit commitment via coordination. In the Overijssel $\mathrm{CNO}$, this commitment resulted primarily from the shared goal: to cooperate in a project with a future national impact in order to increase status. The definition of plans, shared risk and rewards policies has not influenced this commitment. However, the commitment of the participants also increased from informal communication adjustment and communication-oriented coordination, i.e., they negotiate over joint plans, task distribution, governance or resources allocation in formal and informal settings considering shared knowledge and shared learning as specific coordination mechanisms.

\subsubsection{B-ITa best practices}

The findings of our fifth case study helped us to confirm the utility of 15 process areas (see Figure 7.4) which were indeed performed in the Overijssel CNO. These findings can be presented in the form of best practices that can be used by CNOs 


\subsection{CASE STUDY 5: REGIONAL GOVERNMENT NETWORK}

when striving for B-ITa. The term 'best practices' requires some explanation. Best practices, in this dissertation, are validated processes that stand for effective ways to achieve a specific objective (in our case, to achieve B-ITa). Thus, B-ITa best practices are B-ITa processes that have been justified on the basis of evidence provided by verifiable observations (e.g. by a case study) and, therefore, can be presented as general guidelines. However, we make the explicit note that deciding what is "best" is not easy. Best practices can vary over time and from place to place, as new evidence emerge. Moreover, what is "best" also depends on what the $\mathrm{CNO}$ wants and on its context. In the following, we list the identified B-ITa best practices.

1. Define a blueprint of how the collaborative networked organization works, describing how different variables of the collaboration fit together as a system to help creating value for each participant (Business Model Definition - BMD - and Organizational Structure Definition - OSD - process areas).

2. Structure the priorities and allocation of resources and decision rights to create accountability, and to ensure that activities are performed in conformity with policies and procedures (Roles and Responsibilities Specification - RRS - and Governance Structure and Compliance - GSC - process areas).

3. Create a snapshot of the existing ISs and data, assessing what the current state of the CNO is concerning standards and ISs (Standards and Principles Definition - SPD - and Baseline IS Architecture Description - BAD - process areas).

4. Define the ISs needed to support the desired To-Be state of the IS architecture (IS Architecture Target Formulation - ATF - process area).

5. Create a snapshot of the existing processes analyzing what the current state of the collaboration, and maintaining a repository of assets and environment standards (Baseline Process architecture Description - BPD - process area).

6. Evaluate, select and design processes needed to support the desired To-Be state of the process architecture taking into account business and strategy drivers (Process Architecture target Formulation - PAF - process area).

7. Plan, implement, and deploy process improvements based on a thorough understanding of strengths and weaknesses of the CNO's processes and process assets (organizational Process Focus Planning - PFP - and Process Portfolio Management - PPM - process areas).

8. Coordinate the work using Direct Supervision (DTS process area), communication (Informal Communication Adjustment - InCA - and CommunicationOriented Coordination - COC - process areas), and Standardization (STD process area). 


\section{CHAPTER 7. B-ITA PROCESSES}

The process areas covered by these B-ITa best practices formed the first set of processes included in the ICoNOs MM. However, we make the note that they are not the final B-ITa process areas. In the next chapter, we present the ICoNOs MM together with the process areas that ultimately are included in it.

\subsubsection{Validity threats}

In Chapter 3 we introduced the concepts of construct, internal and external validity. In the next paragraphs, we describe how we addressed the threats to validity (in each of these three validity types) in the case study presented in this chapter.

\subsubsection{Construct validity}

The fact that the gathered data were situation-specific and therefore impossible to replicate, together with the possibility of researcher's bias, were threats to construct validity in this case study. We dealt with these threats by (i) data triangulation in the data collection phase (i.e., we used professionals, meetings, documents, and illustrative materials); (ii) using verbatim interview transcripts and notes of observations in order to allow cross checks of particular sources of data; and (iii) checking the observations, and interpretations of them, with participants (member checking) and researchers (peer debriefing) as a form of triangulation.

\subsubsection{Internal validity}

In order to assure the quality, rigor and trustworthiness of the collected data such that it assures that the results of the research were caused by the phenomena under investigation and not by incidental influences (see Chapter 3), we addressed internal validity in this case by reflexivity and low inference/rich descriptions.

\subsubsection{External validity}

As this case was a single interpretative study - since we are studying one (constantly changing) organization, it can be argued that we cannot generalize results. We consider two kinds of external validity threats: (i) single case studies cannot be used to generalize, and (ii) interpretative research cannot generalize. We took some steps to counter them. First, according to Yin [348], to be able to generalize to valid statements from a case study, we need to use multiple sources of data. This can help to ensure the quality of the final conclusions. Hence, we responded to the first validity threat by using professionals, meetings, documents and illustrative materials as sources of evidence. To confront the second validity concern, we based our analysis process on theories, frameworks, and principles developed by case study research methodologists (e.g., [165, 175, 325, 348]). In summary, they claim that the generalization from empirical descriptions to theoretical statements is possible and valid. We therefore abstracted from the data general principles that could throw light on other similar situations (see Section 7.4.3). 


\subsection{Summary}

This chapter presented the literature review and the case study we conducted to identify B-ITa process areas. We used several theories and models, developed elsewhere, to recollect constructs that had a relation with the four B-ITa domains that the ICoNOs MM covers. The mapping of those constructs (see Figure 7.2) helped us to identify a first set of processes related to B-ITa in CNOs: seven partnering structure process areas, nine IS architecture process areas, nine process architecture process areas, and five coordination process areas. Our execution of the case study brought real-life evidence to support the inclusion of the identified process areas presented in Figure 7.1 in the B-ITa domains, within a specific level, of the ICoNOs MM.

We make the note that this study is not enough to overcome external validity concerns that a case study is exposed to. For example, the case site investigated was not randomly sampled. Therefore it is not possible to generalize our findings to a wider population of organizations. However, we generalized the case study findings to theoretical statements (i.e, to the B-ITa best practices presented in Section 7.4.3) and, thus, part of the results can be drawn on by others who work in organizational contexts where B-ITa plays a valuable role.

The results gained through this study let us increase our knowledge of the first set of process areas included in the ICoNOs MM. It served to establish the soundness of the ICoNOs MM and, specifically, of those B-ITa process areas. The final process areas included in our model are presented in the next chapter. 



\section{8 The ICoNOs MM}

In Chapter 7 we presented the process areas included in the ICoNOs MM. Similarly to CMMI [57], these process areas have specific and generic goals, which the activities in each process area are supposed to achieve. Specific goals (SGs) describe characteristics that must be present to satisfy a particular process area. That is, they are specific for this area. Generic goals apply to all process areas, although their instantiation for each process area can differ. For example, a CMMI generic goal is 'the process is institutionalized as a defined process'. This goal can be applied to all processes. Our ICoNOs MM will incorporate all generic goals of CMMI (see Appendix B). The specific and generic goals are respectively decomposed in specific and generic practices. Practices describe what a CNO may implement to achieve the goals. These practices are expected and not mandatory (see Section 4.2.2 in Chapter 4). This means that one can implement alternative practices to replace the specific and generic practices that the ICoNOs MM includes. The only condition is that the goals must be satisfied, to perform a process, to reach a specific maturity level.

In this chapter, we present the SGs and the specific practices (SPs) of the B-ITa process areas included in the ICoNOs MM. We only introduce the SGs and SPs included in the levels two and three because in these levels is where CNOs can make the most significant improvements. We make an explicit note that, when decomposing the process areas presented in Chapter 7, we have changed the position of some of them. We found that some of the process areas are strongly related to each other. We then decided to re-arrange de position of them, so that some of the original process areas are now presented as goals of, or practices in, another process area. For example, the "Inter-organizational policies definition" and the "Roles and responsibilities specification" process areas at level three of the partnering structure domain have been moved to the "Governance structure and compliance" process area. Figure 8.1 presents the ICoNOs MM including the last version of the B-ITa process areas.

As we have presented in Chapter 7, our position was that it would be practical for CNOs to have a selection of B-ITa processes covered by different models and/or theories in a single model. Therefore, some of the SGs and SPs have been taken, and slightly adapted, from models/theories developed elsewhere. For 
CHAPTER 8. THE ICONOS MM

\begin{tabular}{|c|c|c|c|c|}
\hline & \multicolumn{2}{|l|}{ PARTNERING STRUCTURE } & \multicolumn{2}{|l|}{ IS ARCHITECTURE } \\
\hline 5 & & & $\begin{array}{l}\text { Inter-organizational IS arch. optimization } \\
\text { Risk analysis and mitigation }\end{array}$ & $\begin{array}{l}\text { IOAO } \\
\text { RAM }\end{array}$ \\
\hline 4 & Metric-based roles exploration & MRE & Quantitative IS portfolio management & QPM \\
\hline 3 & $\begin{array}{l}\text { Governance structure and compliance } \\
\text { Service level agreements definition }\end{array}$ & $\begin{array}{l}\text { GSC } \\
\text { SLA }\end{array}$ & $\begin{array}{l}\text { IS requirements management } \\
\text { IS capabilities definition } \\
\text { IS portfolio management }\end{array}$ & $\begin{array}{l}\text { IsRM } \\
\text { IsCD } \\
\text { IsPM }\end{array}$ \\
\hline 2 & Business model definition & BMD & Current IS architecture description & CSA \\
\hline 1 & & & & \\
\hline
\end{tabular}

\begin{tabular}{|c|c|c|c|c|}
\hline & \multicolumn{2}{|l|}{ PROCESS ARCHITECTURE } & \multicolumn{2}{|l|}{ COORDINATION } \\
\hline 5 & $\begin{array}{l}\text { Inter-organizational process optimization } \\
\text { Causal analysis and resolution }\end{array}$ & $\begin{array}{l}\text { IOPO } \\
\text { CAR }\end{array}$ & & \\
\hline 4 & $\begin{array}{l}\text { Organizational process performance } \\
\text { Event logs formal consistency }\end{array}$ & $\begin{array}{l}\text { OPP } \\
\text { EFC }\end{array}$ & Quantitative coordination relation analysis & QRA \\
\hline 3 & $\begin{array}{l}\text { Organizational process focus planning } \\
\text { Target process architecture formulation }\end{array}$ & $\begin{array}{l}\text { PFP } \\
\text { TPA }\end{array}$ & $\begin{array}{l}\text { Standardization } \\
\text { Communication-oriented coordination }\end{array}$ & $\begin{array}{l}\text { STD } \\
\text { COC }\end{array}$ \\
\hline 2 & Current process architecture description & CPD & $\begin{array}{l}\text { Informal communication adjustment } \\
\text { Direct supervision }\end{array}$ & $\begin{array}{l}\text { InCA } \\
\text { DTS }\end{array}$ \\
\hline 1 & & & & \\
\hline
\end{tabular}

Figure 8.1: The ICoNOs MM.

example, the SGs and SPs of the "IS requirements management" process area have been adapted from the CMMI [57], the TOGAF [302] and the work of Sommerville and Sawyer [292]; and the SGs and SPs of the "Current process architecture description" and the "Organizational process focus planning" process areas have (mostly) been taken from the CMMI [57].

We present the SGs and SPs of the B-ITa process areas grouped into the four B-ITa domains. We list the B-ITa process areas in alphabetical order. For each process area, beside the SGs and SPs, we also provide the process areas which it relates to and the meta-level results that the CNO will get at the end, if the process areas are consistently applied. Some SPs are further decomposed in specific tasks (STs). Previous versions of the SGs and the SPs have been published in the proceedings of the 4th IEEE/IFIP International Workshop on Business-Driven IT Management [269] and the 10th IFIP Working Conference on Virtual Enterprises [270].

\subsection{Partnering structure}

\section{BMD 8.1.1 Business model definition}

A partnering structure process area at maturity level 2

The main purpose of the business model definition (BMD) process area is to define a blueprint of how the CNO works, describing how different variables of the collaboration fit together as a whole to help creating value for each participant. By 'variables' we mean the various elements that help the CNO to understand 


\subsection{PARTNERING STRUCTURE}

itself and its environment in order to visualize its current position in the market, to establish where the collaboration wants to be and to identify the way in which value is created, distributed and consumed among the participants.

In the context of our research, a business model represents a description of the CNO presenting the direction and the goals to all participants. The literature provides many definitions of the term 'business model'1. While some of these definitions refer to a business model as a simple statement about what an organization does, other definitions, rather than grossly oversimplifying the term, dive in at a level of complexity that forecloses a blueprint of the organization.

\section{Specific goals and practices}

SG1 Define the CNO overall identity.

The CNO identity includes elements such as the organizational mission, the unique useful internal assets, the product/service that will be offered and the status of the $\mathrm{CNO}$ in the market.

SP1.1 Identify the mission and vision of the CNO.

The mission statement describes both the purpose and direction of the CNO. It is a starting point for defining particular goals. Moreover, the vision statement indicates what the CNO calls 'success' and operationalizes such a success in terms of collaborative B-ITa goals.

SP1.2 Identify unique useful internal assets.

Internal assets include the resources that the collaboration might gather together to carry on its B-ITa operations. This includes for example, intellectual property, organizational resources, financial resources, physical resources, human capital and distribution channels.

SP1.3 Describe the product/service offerings emphasizing any unique features and highlighting any differences with current products/services offerings in the market.

SP1.4 Identify the competitive advantage of the CNO.

According to Riemer and Klein [256], the increasing number of CNOs is supposed to impact entire industries by modifying the way of thinking on competition from a single-organization level to a CNO level. In this scenario, entire CNOs "compete with each other making it necessary for networks to position themselves and to build out a competitive advantage" [256, p. 30] from a CNO perspective. This competitive advantage depends on the ability of the participants to forge close relations with their external environment, as well as within them.

SG2 Identify the external environment.

${ }^{1}$ Please refer to [286] for a a discusssion on this respect. 


\section{CHAPTER 8. THE ICONOS MM}

The external environment identification involves the definition of (i) the market in which the CNO intends to operate, (ii) which the supporting organizations are, and (iii) what the competitors and their main strengths are.

SP2.1 Describe the target market (group of customers) that the CNO has decided to serve - and which it consequently gears its operations and characteristics to.

SP2.2 Define the supporting organizations (e.g. suppliers, technological partners, chamber of commerce, government entities, financial institutions).

SP2.3 Identify the competitors.

The identification of competitors involves to perfom a competitive analysis for the CNO. This analysis can be performed using, for example, the five competitive forces of Porter [238] and the SWOT technique.

SG3 Describe a multi-value configuration.

The multi-value configuration describes the arragement of issues related to investments, costs, and revenue. The main issue to describe is a value model where participants can visualize its current position in the market.

\section{SP3.1 Construct a value model.}

The main objective of a value model is to explore the economic feasibility of the CNO, by focusing on the concept of economic value (money, fee, payment, or any object of value), which is exchanged reciprocally between the participanting organizations [118]. A value model commonly helps to define a cost structure and a revenue model.

SP3.2 Describe the revenue model.

The revenue model describes how the CNO makes money and achieve its sustainability through a variety of revenue flows. According to the type of CNO, the participants can use different types of revenue models (e.g. transaction-based, sponsorships, sales, effectiveness gains, etc.).

SP3.3 Define the cost structure.

Structuring the costs implies to present a clear view on the amount of cash flow, and investments, that need to be generated for supporting the CNO operations [149]. The cost structure details the expenses associated with the revenue flows described in the revenue model.

SG4 Formulate an overall CNO plan.

SP4.1 Describe the specific goals to achieve. 


\subsection{PARTNERING STRUCTURE}

Participants in a CNO can be seen as distinct loosely coupled stakeholders with commonly conflicting interests and goals [71, 318]. However, if they want to collaborate, they need to formulate a clear-enough common goal(s) toward which they strive together. This goal is not necessary the goal of all the participating organizations. The common goal is an agreement among the customer-faced organization and its direct partners. This common goal might include also other participants in the CNO, but not necessarily.

SP4.2 Set a timeframe for achieving the goals.

SP4.3 Define performance indicators.

The performance indicators should include expectations of the stakeholders and the supporting institutions. Indicators must be relevant so that they let to assess the real operations, and reliable so that they do not get affected by organizational changes.

\section{Related process areas}

Refer to the governance structure and compliance (GSC) process area for more information about the property rights distribution on internal assets.

\section{Expected results}

- An understanding of the essential elements that contribute to provide value to all participating organizations.

- A multi-value configuration of the CNO knowing how it will make money and sustain its profit stream over time.

- A competitive strategy and an overall CNO plan by which the CNO will gain and hold advantage over its rivals.

\subsubsection{Governance structure and compliance}

A partnering structure process area at maturity level 3

The main purpose of the governance structure and compliance (GSC) is to structure the priorities and allocation of resources and decision rights to create accountability; and to ensure that activities are performed in conformity with policies and procedures. Luftman [189] has already stated that one of the most significant enablers of B-ITa is to ensure that the people involved in the B-ITa project in a organization formally discuss and review the priorities and allocation of IT resources considering issues as IT/business strategic planning.

However, as our research is focused on operational B-ITa, we consider those aspects only which operationalize organizations in specific actions to perform [335]. Examples of these aspects are the property rights distribution, the decisionmaking procedures, the control and accountability mechanisms, and the formalization of roles and responsibilities of the participating organizations. 


\section{CHAPTER 8. THE ICONOS MM}

\section{Specific goals and practices}

SG1 Describe the overall organizational structure.

The organizational structure description includes to build a CNO chart, to specify the roles and responsibilities, and to distribute the property rights among the participating organizations.

SP1.1 Depict the CNO chart.

The main objective of the CNO chart is to set a framework for reaching decisions among the participating organizations in a timely fashion for conflict resolution. This chart establishes formal channels of communication and determines the placement of power and authority in the collaboration.

SP1.2 Specify the roles and responsibilities.

This specification helps to establish an accountability system in the $\mathrm{CNO}$, which is important because participants can play different roles in a CNO [112, 317, 318]. For example, from specialist, i.e., an organization who performs one or few activities and provides services (e.g., a supplier of technology within a $\mathrm{CNO}$ ), to network integrator, i.e., a dominant participant who attempts to coordinate the activities performed by everyone in the CNO.

ST1.2.1 Identify the CNO actors (participants).

ST1.2.2 Define provider-specific activities.

ST1.2.3 Formalize roles and action plans.

ST1.2.4 Distribute control and accountability.

SP1.3 Define the distribution of property rights.

"A property right is the exclusive authority to determine how a resource is used" [153, p. 82]. Commonly, property rights include (i) exclusive rights to the use of a resource, (ii) exclusive rights to the services of a resource, and (iii) rights to exchange the resource at mutually agreeable terms [153]. The property rights distribution helps to improve the use of resources and to prevent opportunistic behavior.

SG2 Define the inter-organizational policies.

These policies are the rules that govern the collaborative activities of the CNO. As participants can deviate from the expected behavior, e.g., they could behave opportunistically, the CNO needs instruments to control the behavior of the participants. Such policies help (i) achieve trust and commitments, and (ii) regulate issues as the agreements on information sharing and the setting up of proper incentives and measures for right and wrong behaviors, respectively [278].

SP2.1 Formulate the business rules. 


\subsection{PARTNERING STRUCTURE}

The business rules describe the ultimate handles with which the CNO is able to guide and control its operations. "In fact, the business rules are the means by which an organization implements competitive strategy, promotes policy, and complies with legal obligations" [322, p. 4].

SP2.2 Define shared risks and reward policies in order to strengthen the collaboration among the participating organizations [127].

SP2.3 Specify decision-making procedures.

These decision-making procedures define clear standard criteria for decision making in the CNO. Their specification assures that decisions are based on the best available evidence, take into account the appropiate frameworks and comply with requirements.

SG3 Define the CNO directions using the overal CNO plan as basis.

SP3.1 Build a program activity architecture.

A program is a serie of coordinated, related projects that continue over an extended time. The program activity architecture is an inventory of the CNO general programs based on the target process architecture. The program activity architecture depicts the logical relationships between each program and the participating organizations in the CNO.

SP3.2 Set priorities and plans for how to implement the long-term directions set up in the programs.

SP3.3 Develop a performance measurement framework.

This framework is a collection of the intended results of the CNO and its programs. It sets out the expected results to be achieved and the outcomes to be produced identifying the performance indicators used to assess the programs' progress.

SG4 Ensure programs compliance.

Compliance consists of ensuring that the programs undertaken agree with the inter-organizational policies, the service level agreements, and the IS requirements of the CNO. Enforcing compliance means to ensure the CNO adheres to the 'regulations' stated in the CNO itself.

SP4.1 Identify programs and control objectives.

SP4.2 Confirm adequacy and solve discrepancies.

\section{Related process areas}

Refer to the communication-oriented coordination (COC) process area for more information about the formal communication channels. 


\section{CHAPTER 8. THE ICONOS MM}

Refer to the business model definition (BMD) process area for more information about the construction of a value model which help to idenfity CNO actors (participants) and provider-specific activities.

Refer to the service level agreements definition (SLA) process area for more information about the use of property rights for defining service level agreements.

Refer to the business model definition (BMD) process area for more information about the overal CNO plan required for defining the CNO directions.

Refer to the target process architecture formulation (TPA) process area for more information about the To-Be state of the process architecture which is useful to build a program activity architecture.

Refer to the IS requirements management (IsRM) process area for more information about the IS requirements required for properly compliance.

\section{Expected results}

- Decision-making groups with clear designated focus for accountability.

- B-ITa improvements according inter-organizational policies and specific work directions.

- Met deadlines and low organization's cost of compliance, assuring that 'right' things happen the 'right' way.

\section{SLA 8.1.3 Service level agreements definition}

A partnering structure process area at maturity level 3

The main purpose of the service level agreements definition (SLA) is to describe the agreements on the deliverables, quality, and fitness-for-purpose of services that have an impact on the work of each participating organization.

One of the most important trends in the service management discipline is the shift of the goal of service level agreements from being a financial contract only towards an instrument to manage expectations and the quality of services [25]. Service level agreements should help to meet or surpass business and IT service levels, while identifying gaps in their achievement. Given this trend, and to reach the purpose of the SLA process area, it is important that the identification of needs, the design, implementation and learning of the service process, and the work itself are considered as a continuous operational process [218].

\section{Specific goals and practices}

SG1 Negotiate the agreements. 


\subsection{PARTNERING STRUCTURE}

The negotiation of the service level agreements involves the definition of performance thresholds and measurable commitments for each agreement before starting formally documenting it.

SP1.1 Determine performance thresholds.

Performance thresholds are the minimum acceptable expectations to assure quality in the product or services offered by the CNO. They are usually determined in terms of response times, throughput, and resources utilization [53]. Their definition needs to have a clear view of the current and target architectures of ISs and processes.

SP1.2 Define measurable commitments.

This specific practice is important because a SLA that is specified in measurable terms increases its understanding [25] among the participating organizations supporting the possible future consensus building processes $[117,145]$ regarding the products/services offered.

SP1.3 Document the agreements ${ }^{2}$.

SG2 Describe SLA control issues.

The purpose of the description of control issues is to verify (and implicitly also to manage) the SLAs in a way that they are properly coordinated and met.

SP2.1 Agree on escalation procedures.

Escalation procedures are processes established to define the steps taken when service levels do not meet the agreements. "This may involve determining fault for missed measures, reporting problem resolution within a specified time" [308, p. 114]. Escalation procedures are constitutionalized in the $\mathrm{CNO}$ in order to warn it of any risks so that actions can be taken immediately.

SP2.2 Establish penalties and incentives.

Penalties and incentives are tools that, used wisely, can contribute to create effective and succesful CNOs [109]. To work properly the penalties have to be 'correct' established and the "incentives must be firmly aligned with the underlying business objectives" [126, p. 4].

\section{Related process areas}

Refer to the current IS architecture description (CSA), IS portfolio management (IsPM), current process architecture description (CPD), and target process architecture formulation (TPA) process areas for more information about the current and target architectures of ISs and processes.

${ }^{2}$ For information about SLAs documentation please refer to [17, 25]. 
Expected results

- Specification of results (not only of efforts) to meet particular service levels.

- Understanding of the needs and priorities of the CNO, and its expectations of the capabilities of each participating organization.

- Reduction of time lost in resolving conflicts regarding the quality of the products/services offered.

\subsection{IS architecture}

\section{CSA 8.2.1 Current IS architecture description}

An IS architecture process area at maturity level 2

The main purpose of the current IS architecture description (CSA) is to create a snapshot of the existing ISs and data, assessing what the current status of the CNO is concerning information systems. In general, and based on Hoque's work [138] and the TOGAF model [302], this description of current ISs has three purposes: the first is to help elaborate on ISs functionality, or how requirements are supported by the components that make up each of the ISs. One of the most important challenges of B-ITa is that processes and ISs rarely match one to one, e.g., a business process may need multiple ISs and vice versa.

The second purpose is to help integrate ISs. It is necessary to define where information must be shared at an IS level, and to assure new ISs are interoperable with existing ones. This is important when conducting gap analysis to guarantee new ISs can share vital information with legacy ISs.

The third purpose is to visualize how the ISs behave in specific circumstances.

\section{Specific goals and practices}

SG1 Characterize the existing software applications.

The ISs characterization means to describe the qualities of the ISs [90]. These ISs qualities include, for example, whether (i) the ISs perform as intended in all circumstances, (ii) their code is well documented for future reference, (iii) standards of architecture and modularity have been met, (iv) the ISs are accepted by all users. The level of detail for the description depends on the extent to which existing ISs could be carried over into the target IS architecture, and on whether current ISs descriptions exist.

SP1.1 Make an inventory of the current shared ISs of the entire CNO.

SP1.2 Describe what the ISs do (not how they do it) and their status (e.g., planned, almost finished, operational, obsolete).

SG2 Build the software application model. 


\subsection{IS ARCHITECTURE}

The main objective of the software application model is to define the stakeholders (owners/users) of the ISs and to "generate specifications of the functions of the application behavior, including the application's control flow, data dependency, domain decomposition, ... the weight distribution over the domain" [143, p. 15] and the processes and organizational units supported.

SP2.1 Define the stakeholders (owners/users) of the ISs.

SP2.2 Identify the organizational units supported.

SP2.3 Identify the processes supported.

SP2.4 Illustrate how data flows throughout the ISs [138].

SP2.5 Describe the precedent and successor ISs.

SG3 Define the IS standards and principles.

According to TOGAF [302], standards and "principles are general rules and guidelines, intended to be enduring and seldom amended, that inform and support the way in which an organization sets about fulfilling its mission". IS standards and principles are therefore structured set of ideas that provide guidance on the use and deployment of ISs [90] in the CNO.

\section{Related process areas}

Refer to the IS portfolio management (IsPM) process area for more information about the target IS architecture and the gap analysis.

\section{Expected results}

- An understanding of the current state of the CNO concerning ISs for planning the target IS architecture and defining specific IS requirements.

\subsubsection{IS capabilities definition}

An IS architecture process area at maturity level 3

The main purpose of the IS capabilities definition $(\mathrm{ISCD})$ is to define the ability of the CNO to operate efficiently by using ISs to achieve congruence with the business environment where it works [260] and to improve its performance [184]. "IS capabilities are those necessary and sufficient to ensure that an organization can exploit changing markets of technology and services - to achieve business advantage through IT over time" [104, p. 20].

IS capabilities involve competencies and routines that complement ISs [6]. In the one hand, competencies refer to technical, business and interpersonal skills embodied in individuals or groups [93]. As individuals and groups interact with 
IS for specific purposes, they learn and build skills and develop competence toward the development/use/management of ISs. In the other hand, routines are activities that help individuals or groups to get skills, e.g., the culture of IS used in an organization.

\section{Specific goals and practices}

SG1 Analyze the IT and business skills of the participants in the CNO.

SG2 Identify the motivating values of the participants.

In his work Fenny and Willcocks [104, p. 17] mention that "today's business systems thinkers or relationship builders were often yesterday's high performers in making technology work". Commonly, people (and organizations) have multiple capabilities. What change over time are their motivations, values or challenges. When collaborating for reaching common goals and serving customers over a period of time, participating organizations in a CNO must identify their motivating values in order to define their IS capabilities as CNO (see next SG).

SG3 Define the IS capabilities of the entire CNO considering the previous two SGs.

\section{Related process areas}

Refer to the standardization (STD) process area for more information about the interpersonal skills when using standardization as coordination mechanism.

\section{Expected results}

- A successful exploitation of ISs using core IS capabilities in order to create competitive advantage.

\section{\begin{tabular}{lll}
\hline IsPM & 8.2.3 & IS portfolio management
\end{tabular}}

An IS architecture process area at maturity level 3

The main purpose of the IS portfolio management (IsPM) is to create the right mix of ISs investments to properly use limited resources while providing the maximum business benefit. IS portfolio management is gaining interest as organizations look for ways to reduce IT costs. It helps to judge ISs based on the role that participating organizations expect they fulfill in the CNO. "There are many ways of classifying information systems, but for investment appraisal purposes the role they play in the business and the contribution they are expected to make should be the key parameters for such a classification" [327, p. 84]. 


\subsection{IS ARCHITECTURE}

According to the Application Service Library (ASL) [311], the main goal of the IS portfolio management is to outline the significance and performance of the existing ISs, to translate the goals and requirements of an organization into possible target ISs to set an IS investments plan. Rationalizing new ISs designed to provide new value to the $\mathrm{CNO}$ is a process that requires considerable effort.

\section{Specific goals and practices}

SG1 Define the target IS architecture.

The definition of the target IS architecture covers aspects as the identification of the improvements, the construction of the target ISs model and the definition of new ISs investments within the CNO.

SP1.1 Identify improvements and/or adjustments based on the requirements derived from analyzing the goal(s) and the processes of the CNO, and the relations between the participating organizations.

SP1.2 Build the target IS model.

ST1.2.1 Define the stakeholders (owners/users) of the target ISs.

ST1.2.2 Identify the organizational units supported.

ST1.2.3 Identify the processes supported.

ST1.2.4 Illustrate how data flows throughout the target ISs [138].

ST1.2.5 Describe the precedent and successor ISs.

SP1.3 Validate and verify the target IS architecture (gap analysis).

Verification and validation of the IS architecture assures that ISs satisfy the requirements of the business in the $\mathrm{CNO}$ - it is a crucial SG in our B-ITa context since it explicitly helps to guarantee what we mention in our B-ITa definition (see Chapter 2). Validation demonstrates that the ISs will fulfill their intended use; whereas, verification addresses whether the ISs properly reflect the specified requirements (see "Validation" and "Verification" process areas of the CMMI [57]).

ST1.3.1 Select the method to be used for the verification and validation (e.g., test, analysis, inspection, demonstration, or simulation).

ST1.3.2 Establish the verification/validation environment, procedures and criteria.

ST1.3.3 Perform verification/validation and analyze the results for making adjustments in the target IS architecture.

SP1.4 Define the new investments on ISs.

ISs investments can be defined by calculating and listing the operation and the actual cost of investments per IS (for past, present and future situations). "This requires a sophisticated IT controlling model that will accurately assign direct and indirect cost and compile a cost scheme based on application-centric services" [257, p. 374] as presented in Zarnekow et al. [350]. 


\section{CHAPTER 8. THE ICONOS MM}

SG2 Define insights and facts for decision making.

The definition of insights and facts for decision making involves to define key application metrics and to conduct risk analysis in order to identify currect and future (money-spinner) ISs that are stable, matured and have very low risk.

SP2.1 Define key application metrics in order to identify the ISs performance in the CNO. Examples of these key metrics are: adaptability, flexibility, availability. They are commonly subjective characteristics and, therefore, cannot be measured directly. However, these metrics can be defined using other parameters such as transaction throughput and resource utilization.

SP2.2 Conduct risk analysis to determine the risk associated with their ISs and to ensure that IS projects come in on time and under budget. "Risk analysis relies heavily on an understanding of ... the business model supported by the software" [321, p. 80]. CNOs can use risk analysis to ensure the profitable execution of their core goals [140].

SG3 Develop a plan to obtain all participants' buy-in.

The plan must cover (i) recommendations on maintaining, upgrading, replacing, or retiring the ISs of the CNO to optimize its IS portfolio, and (ii) decisions on which applications are worth of immediately action. These recommendations and decisions should be balanced among time, budget, human resource constraints, saving money, and/or setting the CNO on a transformative path [225] in order to present a streamlined IS portfolio and a view into the value delivered to the CNO. This helps to obtain the commitment of all participants.

\section{Related process areas}

Refer to the IS requirements management (IsRM) process area for more information about the IS requirements required to identify improvements.

Refer to the business model definition (BMD) process area for more information about the business model required when performing a risk analysis.

Refer to the current process architecture description (CPD), and target process architecture formulation (TPA) process areas for more information about the current and target process architectures required when building the target IS model. 


\subsection{IS ARCHITECTURE}

\section{Expected results}

- Well-balanced IS investments in terms of size, risk and success of the CNO B-ITa project.

- Qualitative and quantitative cost, benefit and risk considerations into decisionmaking.

- The identification of (i) improvements to established ISs and (ii) new ISs to engineer.

- Reduction of overlapping/duplicative ISs functionality.

\subsubsection{IS requirements management}

An IS architecture process area at maturity level 3

The main purpose of the IS requirements management (IsRM) is to manage the changing IS requirements during their engineering process and the development of the required ISs. The management of requirements is an essential element of ISs development to ensure B-ITa in the CNO. As the IS architecture of the CNO is more complex than the IS architecture of a single organization, the management of the IS requirements becomes increasingly challenging. The IsRM has in general to do with all the processes involved in changing IS requirements.

\section{Specific goals and practices}

SG1 Obtain an understanding of IS requirements based on the target IS architecture of the CNO.

SP1.1 Identify and record requirements.

ST1.1.1 Establish scenarios (presenting sequences of events that might occur in the use of the ISs) which are used to make explicit some of the needs of the entire CNO.

ST1.1.2 Establish a definition of required functionality.

ST1.1.3 Record the identified requirements.

SP1.2 Assess the impact of requirements.

According to CMMI [57], the objectives of this assessment is to determine whether the requirements are necessary and sufficient to meet the objectives, and to identify candidate requirements for IS that will satisfy stakeholder needs, expectations, and constraints. This assessment is performed "to determine what impact the intended operational environment will have on the ability to satisfy the stakeholders' needs, expectations, constraints, and interfaces. Considerations, such as feasibility, mission needs, cost constraints, potential market size, and acquisition strategy, must all be taken into account, depending on the product context" [57, p. 397]. 


\section{CHAPTER 8. THE ICONOS MM}

SP1.3 Determine requirements priorities in order to define which to implement and which to defer.

When prioritizing requirements, one must identify which the global, and the most volatile, requirements are. Global requirements, in a CNO context, are requirements that set out desirable properties of new collaborative ISs (thus, no single-organization-based ones). "Volatile requirements are those requirements which are the most likely to change ... All system requirements change but it is generally the case that some requirements change more frequently that other, more stable, requirements" [292, p. 249].

SG2 Obtain commitment to requirements of all participating organizations in order to ensure that they are aware of the IS requirements and guarantee its participation in the management process.

SG3 Define policies for requirements management.

Requirements management policies cover goals for requirements management, the procedures which should be followed and the standards which should be used to implement the procedures in particular situations [292].

SG4 Use a database to manage requirements.

Sommerville and Sawyer [292] advice to establish a requirements database and store the requirements as entries in this database rather than maintaining requirements in text documents. This database "makes it easier to maintain links between individual requirements and to search for and abstract related groups of requirements" [292, p. 236].

\section{Related process areas}

Refer to the IS portfolio management (IsPM) process area for more information about the target IS architecture.

Refer to the business model definition (BMD) process area for more information about the mission, cost structure, potential market and strategic plan required when assessing the impact of the IS requirements.

Refer to the standardization (STD) process area for more information about the way in which requirements management standards can be used as coordination mechanisms.

\section{Expected results}

- Reduction of ISs development schedules extensions and costs incurred for rework of the design and implementation of the IS architecture to accommodate requirements changes. 


\subsection{Process architecture}

\subsubsection{Current process architecture description}

A process architecture process area at maturity level 2

The main purpose of the current process architecture description (CPD) is to create a snapshot of the existing processes, and maintaining a repository of measures and assets. Processes are the vehicle through which an organization delivers its products or services. They are the structure for action that enables the definition of coordination mechanisms [278].

In a CNO context this architecture takes a vital role because participants need to integrate both IT processes and business processes when they have to define and manage the collaborative processes for reaching the common goal(s) and for exchanging information. With such process architecture, they can formalize what processes will be performed in collaboration, and what processes will be owned by each organization [47].

Similar to the CSA process area, the level of detail for the description of the current process architecture depends on the extent to which existing processes could be carried over into the target process architecture, and on whether current processes descriptions exist.

\section{Specific goals and practices}

SG1 Characterize the existing processes.

SP1.1 Make an inventory of the current relevant processes (participant-specific and collaborative processes to reach the goals).

SP1.2 Build a process model.

SG2 Establish the CNO's measurement repository.

"The repository contains both product and process measures that are related to the organization's set of standard processes. It also contains or refers to the information needed to understand and interpret the measures and assess them for reasonableness and applicability" [57, p. 226].

SP2.1 Determine the needs for storing, retrieving, and analyzing measurements.

SP2.2 Define and agree upon a common set of process measures.

"The measures in the common set are selected based on the organization's set of standard processes. They are selected for their ability to provide visibility into process performance to support expected business objectives. The common set of measures may vary for different standard processes" [57, p. 227].

SP2.3 Design and implement the measurement repository. 
SP2.4 Make the contents of the measurement repository available for use by the CNO.

SG3 Establish the CNO's process asset library.

The "process asset library is a collection of items maintained by the organization for use by the people and projects of the organization. This collection of items includes descriptions of processes and process elements, descriptions of lifecycle models, process tailoring guidelines, process-related documentation, and data" [57, p. 219].

SP3.1 Design and implement the organization's process asset library.

SP3.2 Specify the criteria for including items in the library.

SP3.3 Specify the procedures for storing and retrieving items.

SP3.4 Have the selected items entered into the library.

SP3.5 Make the items available for use by the CNO.

\section{Related process areas}

Refer to the target process architecture formulation (TPA) process area for more information about the target process architecture of the CNO.

Refer to the organizational process focus planning (PFP) process area for more information about the deployment of standard processes.

Refer to the standardization (STD) process area for more information about processes standardization.

\section{Expected results}

- An understanding of the current state of the CNO concerning processes for formulation of the target process architecture.

\section{\begin{tabular}{l|ll}
\hline PFP & 8.3.2 & Organizational process focus planning
\end{tabular}}

A process architecture process area at maturity level 3

The main purpose of the organizational process focus planning (PFP) is to plan, implement, and deploy process improvements based on a thorough understanding of strengths and weaknesses of the CNO's processes and process assets. According to CMMI, "candidate improvements to the organization's processes and process assets are obtained from various sources, including measurement of the processes, lessons learned in implementing the processes, results of process appraisals, results of product evaluation activities, results of benchmarking against 


\subsection{PROCESS ARCHITECTURE}

other organizations' processes, and recommendations from other improvement initiatives in the organization" [57, p. 241].

Process improvement is a well-known concept that commonly is discussed at the level of single enterprises. However, there is still a lack of empirical grounded contributions on the concept of process improvement in CNO settings [205]. Collaborative improvement of processes takes place within the context of the requirements of the $\mathrm{CNO}$ and is used to address the shared goals of the participating organizations and to enhance the CNO overall performance. "The organization's planning for process improvement results in a process improvement plan" [57, p. $241]$.

\section{Specific goals and practices}

SG1 Determine process improvement opportunities.

The process improvement opportunities identification involves the definition of the organizational process needs, the appraisal of the processes and the identification of unique process improvements that specifically address the CNO's needs.

SP1.1 Establish the CNO process needs.

The processes of the CNO operate in a business context that must be perceive and comprehend from an IT perspective. The CNO's goals, requirements, and constraints determine the needs and objectives for its processes. Aspects related to finance, technology, quality, human resources, and marketing are commonly important process considerations [57]. Following the CMMI recommendations, the CNO process needs and objectives must cover issues as (i) characteristics of the processes, (ii) process-performance objectives, and (iii) process effectiveness.

SP1.2 Appraise the CNO's processes to maintain an understanding of their strengths and weaknesses.

Process appraisals can be conducted in many ways. For example, the appraisal can be based on a process model (such as the CMMI), on a national or international standard (such as ISO 9001 [146]), or on benchmark comparisons with other CNOs.

SP1.3 Identify the CNO's process improvements.

SG2 Plan and implement process improvements.

"Successful implementation of improvements requires participation in process action planning and implementation by process owners, those performing the process, and support organizations" [57, p. 247]. This SG involves to establish and implement a process action plan.

SP2.1 Establish process action plans. 
"Process action plans are detailed implementation plans. These plans differ from the whole organization's process improvement plan in that they are plans targeting specific improvements that have been defined to address weaknesses usually uncovered by appraisals" [57, p. 247]. Similar to the IsPM process area, it is important to obtain the commitment of all participanting organizations in order to increase the likelihood of effective implementation.

SP2.2 Implement process action plans.

SG3 Deploy CNO process assets and incorporate lessons learnt.

Process assets are "artifacts that relate to describing, implementing, and improving processes (e.g., policies, measurements, process descriptions, and process implementation support tools). The term process assets is used to indicate that these artifacts are developed or acquired to meet the business objectives of the organization, and they represent investments by the organization that are expected to provide current and future business value" [ $[57$, p. 545].

SP3.1 Deploy CNO process assets.

Deploying CNO process assets must be performed in a systematically way in order to avoid problems. Some process assets may not be appropriate for use in some parts of the CNO (because their implementation could affect the way that customers received the product/services offerings). "It is therefore important that those that are or will be executing the process, as well as other organization functions (such as training and quality assurance), be involved in the deployment as necessary" [57, p. 249].

SP3.2 Monitor implementation to ensure that the CNO process assets are appropriately deployed to all projects.

SP3.3 Incorporate process lessons learnt into the CNO process assets.

When deploying the process assets, it is important (i) to conduct periodic reviews of the effectiveness and suitability of them with relation to the CNO goals, (ii) to obtain feedback about their use, (iii) to derive lessons learned from defining, piloting, implementing, and deploying them, and (iv) to make available lessons learnt to the participating organizations in the CNO.

\section{Related process areas}

Refer to the current process architecture description (CPD) process area for more information about how the CNO's process asset library can support and can enable the deployment of CNO process assets. 


\subsection{PROCESS ARCHITECTURE}

Expected results

- A CNO culture on process improvements based on process assets.

- Improved processes at organization-wide level through a process focus planning initiative.

- An increasing performance of the $\mathrm{CNO}$ as a whole, and of the participating organizations themselves.

\subsubsection{Target process architecture formulation}

A process architecture process area at maturity level 3

The main purpose of the target process architecture formulation (TPA) is to identify and design the processes needed to support the desired To-Be state of the process architecture taking into account IT and business drivers of the CNO. This involves establishing general tailoring guidelines, identifying external input and output, gathering information about product/service offerings, defining the most important support activities, and building the target process model.

The objective is to formulate the best suited processes scenario that can help to offer the CNO products/services in order to compete effectively in its market.

\section{Specific goals and practices}

SG1 Establish general tailoring guidelines.

Tailoring criteria and guidelines are used for defining new processes required for properly B-ITa in the CNO. Following the CMMI recommendations, these tailoring criteria and guidelines must describe (i) how the CNO's set of standard processes and CNO process assets are used to define target processes, (ii) mandatory requirements that must be satisfied by the target processes, and (iii) procedures that must be followed in performing and documenting process tailoring.

SG2 Identify external interfaces.

By 'external interfaces' we mean the products/services offered by external organizations that are input of the CNO processes. This identification of external interfaces can be started by listing all products/service offerings created by the CNO processes to be defined in the target process architecture (i.e., all process outputs) and all products/services required to perform those CNO processes (i.e., all process inputs). This decomposition of processes is deferred until after the CNO has identified the required external input and external output products/services.

SG3 Gather information about product/service offerings.

Except under the circumstances that the CNO is already familiar with the processes to be defined, the $\mathrm{CNO}$ needs to gather information from existing 
documentation and/or interviews with specific process-related owners about the products/services the $\mathrm{CNO}$ will offers and about the activities required for making the $\mathrm{CNO}$ offerings available.

SG4 Build the target process model.

SP4.1 Create a high-level graphical model to facilitate organization of the processes description and their review.

SP4.2 Define and include roles and information related to the processes in the target process model.

It is important to extend the target process model further to include specific process-related information and the participating organizations (and personnel) that perform activities in the processes defined. For this purpose, the CNO must build a list of supporting elements (people and information).

SG5 Define the most important activities to support the CNO product/service offerings, and identify related activities. All these activities are the specific actions that compose the processes to be defined.

\section{Related process areas}

Refer to the business model definition (BMD) process area for more information about the description of the CNO products/service offerings required when defining the most important activities for their support.

\section{Expected results}

- An established set of processes for supporting the availability of the CNO products/services offerings.

\subsection{Coordination}

\section{COC 8.4.1 Communication-oriented coordination}

A coordination process area at maturity level 3

The main purpose of the communication-oriented coordination (COC) is to agree on communication channels, shared knowledge and learning in order to respond effectively to immediate client's needs and to determine what future markets will require. The SGs in this process area support participating organizations to dynamically build connections among them that handle a specific portion of a specific shared process [72].

Daneva and Wieringa [72] present a particular example of knowledge sharing as a goal in this process area: COC happens when an organization (i) brings 


\subsection{COORDINATION}

information together based on user's role between the different organizational units, or (ii) calls up customers' purchase history with the organization, external reports and discussion items from other sales and service staff members who have already dealt with the customers.

\section{Specific goals and practices}

SG1 Agree on communication channels and common language.

In CNO settings where different organizations with customized practices and habits must collaborate, agreements on communication channels and common language are one of the most critical issues for enabling complete communications and sustaining the CNO. Communication channels within the CNO can vary from simple e-mail and one-to-one meetings to smallgroup activities, quality circles, and suggestion system.

SG2 Create a shared knowledge considering inside/outside information.

A knowledge sharing culture brings participating organizations to a required level of understanding for working. Important in the creation of this culture are the concepts of inside and outside information of a community in the CNO. "Outside information refers to types of information that outsiders of a community assume is inside information of that same community. Whereas inside information pertains to the particular information that members of a community mutually assume to be possessed by all of them. For example in the establishment of a shared understanding on the basis of personal common ground we can divide participants into friends (insiders) and strangers (outsiders). From the management literature it is known that one of the most important steps in human resource management is to ensure that when people enter the organization, they become insiders and do not consider themselves as outsiders" [332, p. 126].

SG3 Use IT to enable the communication between the participants. The use of IT enables organizations to broaden the possibilities for the manner to organize activities over organizational boundaries [169].

SG4 Ensure that participants use continuous learning to respond to immediate needs.

\section{Related process areas}

Refer to the governance structure and compliance (GSC) process area for more information about the organizational structure (hierarchical channels) and the groups/communities in the CNO that are important for identifying inside and outside information when creating a shared knowledge. 
CHAPTER 8. THE ICONOS MM

\section{Expected results}

- Officially defined pathways for sharing information in the CNO.

\section{DTS 8.4.2 Direct supervision}

A coordination process area at maturity level 2

The main purpose of the direct supervision (DTS) is to oversee the work by specific persons (supervisors) who take the responsibility for the processes, providing instructions to others (supervisees) and tracking the effect of their actions. By effective supervision, the participants in a CNO can properly perform their tasks in order to improve B-ITa processes.

Supervision has to be valued as a process area by the CNO in order to be effective. That is, a CNO must spend resources for training and supporting supervisors. This cost time and, commonly, time is what organizations lack because they are busy in being reactive solving one problematic situation to another. In addition, this lack of time has an impact on the supervision process itself. We believe that real and effective supervision is a result of (i) good planning, (ii) trustful relations between partcipating organizations and among people, (iii) a learning process and (iv) investment on time.

\section{Specific goals and practices}

SG1 Plan the supervision.

Planning is essential to effective supervision [203, 319]. When planning, supervisors must define strategies for providing effective, efficient supervision.

SP1.1 Define the supervisory strategy.

A supervisory strategy includes all planned supervisory activities. It is a dynamic document that must be reviewed and updated frequently. Supervisors must discuss the strategy with all the participating organizations in the CNO as plans are made $[132,199]$.

SG2 Establish a safe and trust relation with the supervisees. In a supervisory context, trust defines the willingness of the supervisor to be impartial and candid, and the supervisees to accept objective comments and reviews.

SP2.1 Meet the supervisees.

SP2.2 Present the supervisory strategy.

SG3 Monitor the ongoing activities and provide instructions to adjust problems. 


\subsection{COORDINATION}

SG4 Build a learning agenda.

"If a supervisory process is to have integrity and add value to the organization, it must include a learning process". The supervisor and the supervisee "need to agree on a plan for improvement for the supervisee. The improvement plan should focus on both the strengths and areas of needed development. A learning agenda might involve taking some assertiveness training courses, reading appropriate books regarding assertiveness, and getting some counseling from a professional" $[264$, p. 5].

\section{Expected results}

- Identification and holding of people accountable to commit upon the shared goals.

\subsubsection{Informal communication adjustment}

A coordination process area at maturity level 2

The main purpose of the informal communication adjustment (InCA) is to adjust and control the work among the participants by informal communication outside the imposed hierarchical constrains for day-to-day operations. Informal communication is more important to B-ITa than commonly is recognized. Communication between the participating organizations following the overall organizational structure described in the GSC process area does not function smoothly as such structure implies.

No organization operates in a completely formal structured environment. "The informal structure can dramatically influence an organization's performance, and can also be strategically utilized" [48, p. 107]. Informal communication supplements the formal process of communication in a CNO by filling the gaps and by helping to create a good environment for cooperation between the participants.

\section{Specific goals and practices}

SG1 Identify the social composition of the CNO.

The social composition identification in the CNO includes the definition of the relationship-based structures, the communities of practice, and the unofficial agreed-on processes.

SP1.1 Define the relationship-based structures.

The definition of the relation-based structures implies to identify and to describe the structure of informal communication in the CNO. Chan [48, p. 107] emphasizes that informal organization structures are important for B-ITa. Various informal "relationship-based structures that transcend the formal division of labor and coordination tasks" 


\section{CHAPTER 8. THE ICONOS MM}

cannot be separated from formal structures as it comprises an integral part of the socio technical system of an organization, and consequently, of the entire CNO. To attend comprehensive B-ITa in the CNO, the participating organizations need to consider behavioral issues related to the GSC and the COC process areas such as informal management and culture.

SP1.2 Establish communities of practice.

Based on Wenger's work [333], we define communities of practice in a $\mathrm{CNO}$ as informal groups of people in the $\mathrm{CNO}$ who share a concern or a passion for something they do and learn how to do it better as they interact regularly. It is trough practices that commonly come from these communities that CNOs can do what they do. "Communities of practice are this key to an organization's competences and to the evolution of its competences" [333, p. 241].

SP1.3 Identify the unofficial agreed-on processes.

Unofficial agreed-on processes promote empathy and mutual understanding. These kind of processes make it easier for the participating organizations to work together in order to facilitate give-and-take social exchanges among them. Unofficial processes can generate creative ideas for collaborative B-ITa that cannot be raised in official negotiations.

SG2 Influence the development of the CNO informal structure. The informal structure is essential for organizational control and stability of the participating organizations $[88,326]$.

SP2.1 Promote extracurricular activities.

ST2.1.1 Plan the extracurricular activities.

ST2.1.2 Encourage the attendance of the participants.

SP2.2 Acknowledge contribution to improvement of informal communication.

\section{Related process areas}

Refer to the governance structure and compliance (GSC) and the communicationoriented coordination (COC) process areas for more information about the organizational structure and the formal communication channels, respectively, that are important to consider when defining relationship-based structures in the CNO. 


\section{Expected results}

- Smooth operations running based on trusting relationships created by the informal communication among participants in the CNO.

- Possible standardization of skills that can result from using communities of practice.

- Innovative ideas and creative solution of conflicts.

\subsubsection{Standardization}

A coordination process area at maturity level 3

The main purpose of the standardization (STD) is to coordinate work and interactions by standardizing the processes, outputs and/or skills among the participating organizations. This is important because commonly the ability to keep high quality levels of service/product offerings, and to undertake more complex practices in any organization, has a direct relation with the ability (i) to develop optimum work processes and (ii) to have high-skilled staff, in order to create effective practices that help to standardize outcomes.

\section{Specific goals and practices}

SG1 Standardize processes.

A common and standardized set of collaborative processes promote the development of ISs and ease agreements between participating organizations in a CNO [92]. To achieve operational excellence and flexibility in a CNO, process standardization is critical. "Critical features of such a standardized process must include common definitions of metrics, common language that maintains the integrity of business rules, process logic and data, and flexibility to rapidly change and configure these processes as business challenges evolve" [249, p. 1].

SP1.1 Identify processes that are candidates for standardization.

SP1.2 Design the general work processes ensuring coordination.

SP1.3 Diffuse the standardized processes between participants.

SG2 Standardize outputs.

Coordination in a $\mathrm{CNO}$ can also be achieved through the standardization of work results [206]. Output standardization concerns with ensuring that the work meets certain standards. Such standards can be budgets, property rights and inter-organizational policies.

SG3 Standardize skills/competences.

When standardizing skills, the CNO depends upon the professional standards that the participating organizations, and specifically people, learn 


\section{CHAPTER 8. THE ICONOS MM}

and maintain as part of the entire CNO. Communities of practice commonly help to keep skills up to date [332]. "A severe coordination problem may occur, however, if the skills of different professionals in the organizations do not match ... It is very important that the professional actors do not only have the technical skills to perform the instrumental acts on an adequate level, but also the communicative skills - and means - to relate to other professionals" [332, p. 130].

SP3.1 Define skills to standardize.

ST3.1.1 Make an inventory of the participants' skills.

ST3.1.2 Consider the roles of the participants to identify relevant skills categories (interpersonal, task-oriented, intrapersonal).

ST3.1.3 Set behavioral indicators that make skills observable/measurable. It is important to define behavioral indicators of the skills/competences in order to avoid broadly ambiguous skill definitions. "Behavioral indicators are often written as subjective, non-measurable descriptions of desirable behavior instead of objective measurable results required. An example of a behavioral indicator for the competency entrepreneurship taken from an actual competency model is: applies initiative and drive to find and exploit potential sales opportunities" [301, p. 9]. To fend off subjective behavioral indicators and to ensure that skills can be more observable/measurable, Teodorescu and Carl Binder [301] propose an approach starting from the goals of the organization, and working backward to understand what behavior or tasks lead to the achievement of such goals.

ST3.1.4 Establish the importance of each skill to determine, for instance, which ones are frequently used, difficult or critical.

SP3.2 Educate and train participants in the same skills.

SP3.3 Share CNO values and ethical standards that must be taken into account when working for improving B-ITa.

SP3.4 Evaluate the skills/competences.

According to the International Labour Organization ${ }^{3}$, the evaluation of skills/competences involves to establish which skills/competences people possess and compare them against the skills/competences that people must possess to acomplish specific processes. It is important to determine the form and quantity of evidence to be gathered for such an evaluation. Evidence can be collected by different methods, such as observation, role-playing [219], surveys and written tests.

${ }^{3}$ See http://www.ilo.org 


\subsection{COORDINATION}

\section{Related process areas}

Refer to the current process architecture description (CPD) and the target process architecture formulation (TPA) process areas for more information about the AsIs and To-Be state of the process architecture which is useful to identify processes that are candidates for standardization.

Refer to the governance structure and compliance (GSC) process area for more information about property rights and inter-organizational policies which are important for standardizing outputs.

Refer to the informal communication adjustment (InCA) process area for more information about the communities of practice that can help to standardize skills.

Refer to the business model definition (BMD) process area for more information about the CNO identity and overal plan required for setting behavioral indicators in the CNO.

Expected results

- In-time reactions to changing requirements assuring the effectiveness of the reactions themselves and reducing wasted effort. 



\section{Part III}

\section{Validation}





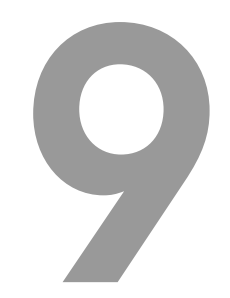

\section{Pilot Assessment}

\subsection{Introduction}

This chapter presents a pilot assessment we conducted to establish the B-ITa maturity of one specific CNO based on the most up-to-date version of the ICoNOs MM (see Chapter 8). Our goal was to evaluate the usability of the ICoNOs MM in a real-life context by using it for this assessment and by analyzing perceptual experiences on the assessment process, the model and the results.

The pilot assessment took the form of a much reduced CMMI assessment. That is, we based our assessment procedure on activities performed in CMMI assessments; however, our assessment procedure is not sophisticated to the extent to which the CMMI assessment procedure is, since we have not yet formalized such a procedure. Unlike the CMMI assessment that is for the most part factual, our pilot assessment also was perceptual (and not only factual). That is, some results are only based on the perceptions of the professionals who have been interviewed or of the author of this dissertation who conducted the assessment, and not on the facts that directly could support the performance of the CNO in a process area.

The pilot assessment appraised the processes of a CNO against the process areas included in the four B-ITa domains, and specifically against the specific goals (SGs) that each of these process areas includes. As it can be seen in the previous chapter, the SGs provide guidelines and focus on results that can be expected to be achieved in a process area. They provide features that a process area must possess in order to yield successful results. Ensuring that the SGs are in place helps to improve the performance and predictability of the process areas included in our model.

In the remainder of this chapter, we specifically describe the $\mathrm{CNO}$ we assessed (Section 9.2), introduce the procedure we followed when conducting the pilot assessment (Section 9.3), and present the assessment results (Section 9.4). Furthermore, we show how we evaluated the usability of our model using a perceptionbased evaluation approach (Section 9.5), and in Section 9.6 we discuss the limitations of the assessment. Finally, Section 9.7 summarizes the chapter. 


\subsection{ICoNOs MM-assessed CNO}

The CNO we assessed using the ICoNOs MM is a collaboration between the three universities of technology in the Netherlands: Delft University of Technology, Eindhoven University of Technology and the University of Twente. The three universities have joined forces in what is known as the 3TU.Federatie (http://www.3tu.nl). The 3TU.Federatie has been officially established in February 2007. The Dutch Ministry of Education, Culture and Science granted a structural subsidy of 6 millions euro for this CNO. According to its founders: "A fully operational 3TU.Federatie will reinforce the dynamics and competitive advantage of the Dutch knowledge-based economy and, by its 'excellent scientific start point', it also will contribute to establish a more innovative environment in the Netherlands and in Europe. This CNO maximizes innovation by combining and concentrating the strengths of all three universities in research, education and knowledge transfer".

An important component of this $\mathrm{CNO}$ is the development of five joint graduate programs. This will enable students to follow master courses offered in the three universities of technology. For example, suppose that a student finds out that a specific course which fits exactly his special field of interest is offered by another university. It is an inviting situation to him. However, when checking the courses time-table, he notices that this specific course starts at 9 a.m., which is not convenient because he first needs to travel three hours by train in order to be present in the course. The 3TU.Federatie will solve this inconvenience by using enterprise service busses (ESBs) that will allow interoperability of the ISs of the three universities without modifying their user interfaces (see Figure 9.1). With such a solution, a student will not need anymore to travel to another university to follow a course. For example, a student at the University of Twente is going to be able to access (online) an IS of the Eindhoven University of Technology by using his trusted graphical user interface (GUI) of the University of Twente. In the 3TU.Federatie, students and teachers will have direct access to the ISs of the three universities in order to follow and impart courses in the three universities, respectively. The 3TU.Federatie is going to offer an educational environment similar to that which has been already offered by big virtual universities in the world - for example, the Canadian Virtual University (http://www.cvu-uvc.ca/) and the Virtual University of the Monterrey Institute of Technology and Higher Education (http://www.tecvirtual.itesm.mx/).

The 3TU.Federatie consists of the following bodies:

\section{Governing Board}

The Governing Board comprises all members of the boards of the three participating universities of technology. On the recommendations of the Executive Committee, the Governing Board determines the long-term plan, the annual plan and the annual report of the 3TU.Federatie.

\section{Executive Committee}




\subsection{ASSESSMENT PROCEDURE}

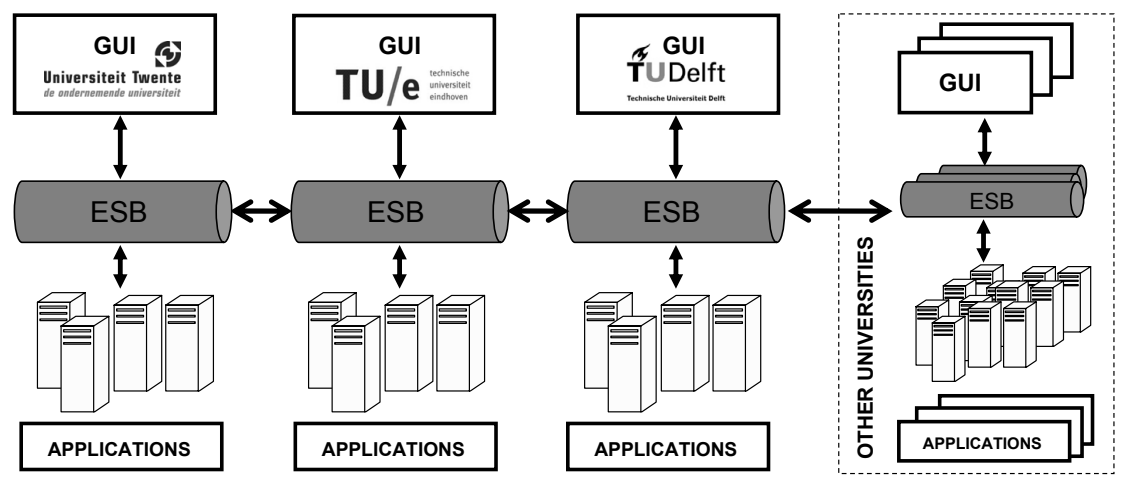

Figure 9.1: The 3TU.Federatie at a glance.

This committee makes decisions on proposals by the 3TU.Institute of Science and Technology, the 3TU.Graduate School, the 3TU.Innovation Lab and the 3TU.Business Operations. It approves plans, budgets and reports by the centers of competence and excellence.

\section{Managing Committee for Research}

This committee is responsible for developing and supervising cooperation and planning of the universities of technology in the area of research.

\section{Managing Committee for Education (3TU.Graduate School)}

This committee is responsible for developing and supervising cooperation and planning of the universities of technology in the area of education.

\section{Managing Committee for Knowledge Valorization}

This committee is responsible for developing and supervising cooperation and planning of the universities in the area of knowledge valorization.

\section{Managing Committee for Business Operations}

This committee is responsible for developing and supervising cooperation and planning of the universities of technology in the area of business operations (including libraries, purchasing, ICT, property).

Centers of Competence and Centers of Excellence

In these centers colleagues of the three universities pool their skills and harmonize their efforts and research priority areas. Professional colleagues from other universities or institutes may contribute as well.

\subsection{Assessment procedure}

In this section, we present how we gathered and analyzed data for this assessment in order to come up with the results and recommendations presented in Section 9.4 


\subsubsection{Data collection}

Similar to SCAMPI (the Standard CMMI Appraisal Method for Process Improvement - http://www.sei.cmu.edu/cmmi/appraisals/), our pilot assessment was based on the information we gathered by means of interviewing professionals from the 3TU.Federatie (we particularly interviewed eight professionals involved in the Architecture Workgroup of the 3TU Graduate School), and of reviewing documents that the interviewees handed to us or that we could find in the $3 \mathrm{TU}$ SURFgroup (i.e., a virtual collaboration environment and shared workspace used by the $3 \mathrm{TU}$ participants to coordinate activities and to share information).

We used semi-structured interviews to gather information related to each process area. The interviews were conducted with 8 persons on a one-to-one basis. The duration of each interview was approximately 1 hour. The interviews were taped. This was done with the consent of each interviewee. The interviews where scheduled with a set of questions to be asked to all interviewees ${ }^{1}$. However, we could pursue relevant information through additional questions that followed up. That is, we had the opportunity to change focus "on the fly" to get more information related to practices that were described by the interviewees in detail. Each question was designed to measure the level of maturity associated with each B-ITa process area. The questions were supposed to be answered in a similar order by all interviewees to make a form of comparison between answers possible. Similar to the fifth case study presented in Chapter 7, the interviews conducted in the pilot assessment were theory-driven. To provide awareness of the ICoNOs MM to the interviewees, we started each interview explaining the "big picture" of the model, and what we meant with the B-ITa term. This made them understand our research context and concepts, which in turn called for clarifications in their own thinking to provide rich explanations. We make the explicit note that, in most of the interviews, the interviewees widely expressed their ideas about the B-ITa domains in the 3TU.Federatie covering answers to most of the questions we have planned to ask. Their participation as interviewees for assessing B-ITa in the 3TU.Federatie was active. In six of the eight interviews, the interviewees additionally provided us with documents that served as evidence of results expected in several SGs. For example, when we asked questions related to the "Governance structure and compliance" process area, one of the interviewees presented to us a document describing a complete governance model that was intended to be implemented in the 3TU.Federatie.

After conducting the interviews, a brief document review was also performed to achieve greater coverage when paired with the interviews and to find additional evidence. We were invited to be members of the 3TU SURFgroup in order to have access to several documents that could be useful for the assessment. The author of this dissertation spent 10 hours browsing and reviewing different documents that, according to their names and descriptions, we considered related to the SGs and process areas included in the ICoNOs MM. This pattern of triangulating multiple data types supported the strength of judgments made at the conclusion

${ }^{1}$ The questions can be found in Appendix D (in Dutch). 


\subsection{ASSESSMENT PROCEDURE}

of the pilot assessment.

\subsubsection{Analysis process}

The data obtained from the interviews and the documents was analyzed by the author of this dissertation to establish the perceived maturity of each B-ITa domain. As in the previous case studies presented in this dissertation (see Chapters 6 and 7), we used interpretation from an hermeneutic perspective (see Chapter 3) to analyze the data. In this case, the hermeneutic perspective helped us to consolidate information and to understand the 3TU.Federatie and its operational B-ITa context. Figure 9.2 presents the analysis process we followed for the assessment.

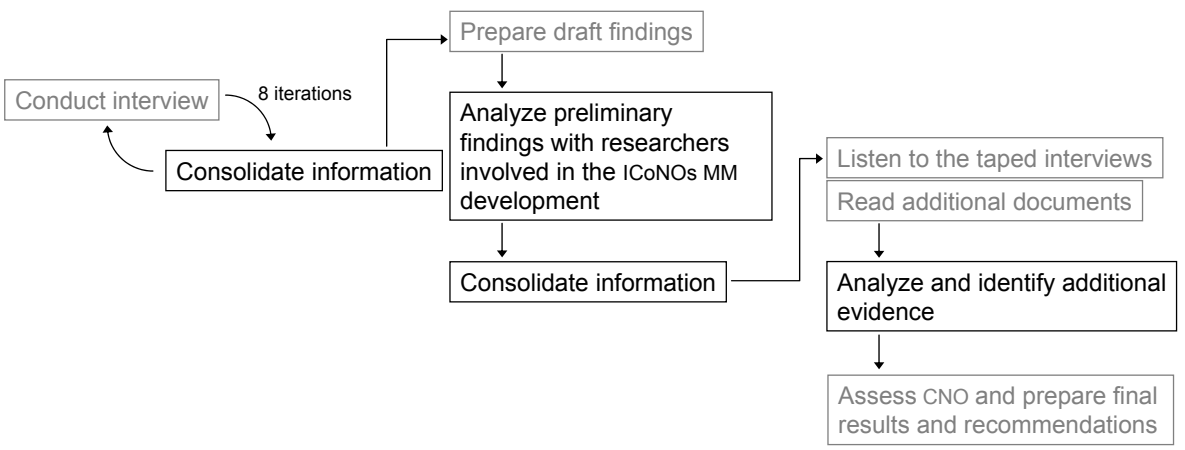

Figure 9.2: Continuous consolidation analysis process.

After conducting each interview, we analyzed the collected information (questionnaire responses and documentation) to focus the further interviews on those aspects that were still not clear to us, and on some questions that were still not answered, for continuous consolidation and feedback.

We prepared a draft of the findings in order to discuss unclear perceptions of specific aspects with the other researchers who were involved in the development of the ICoNOs MM. One of these unclear aspects was the process architecture in the 3TU.Federatie. While interviewing, all interviewees' answers regarding process architecture were consistent: "the 3TU.Federatie has no collaborative processes. We do not need to define new processes in the context you have explained. What we did define are services that each of the universities must offer. We are going to offer the same services to the students, but we have freedom to define our own processes for providing such common services". At first sight, we thought that process architecture was a B-ITa domain that did not apply to the 3TU.Federatie. However, when reading a document that one of the interviewees handed to us for obtaining additional information regarding those services, we found that the services where derived and operationalized from the description of general processes that such services must support. In this respect, we found that the 3TU.Federatie is similar to the Ontarion CNO case presented in Chapter 6. That is, for some pro- 
cesses in the 3TU.Federatie, multiple channels of service provisioning must exist. The defined services should be delivered equally well by the three universities of technology. However, the service delivery process can be independently established. The 3TU.Federatie allows variability of cross-organizational processes which compose the shared process architecture. The universities can keep variety in terms of service-provisioning processes but they must know why they do it and why their processes vary.

Furthermore, we listened to the taped interviews and we reviewed several documents shared in the 3TU SURFgroup. This was done in order to find and structure any additional evidence supporting the performance of SGs and process areas before writing the wrap-up assessment results and recommendations.

\subsection{Results and recommendations}

After analyzing the gathered data, the next step was to "map" the practices within the 3TU.Federatie to the SGs included in the ICoNOs MM. The questions used in the interviews were designed such that this mapping could be easily done (see Appendix D). Table 9.1 presents a summary of the assessment results. In this table the acronyms PS, IS, PA and CO stand for partnering structure, IS architecture, process architecture, and coordination, respectively.

Similar to the case study presented in Chapter 7 , if the interviewees answered positively providing explanations of a specific process area and presenting documents as evidence, then we considered that such a process area was performed in the 3TU.Federatie - and we put the checkmark $\checkmark$ in the PERFORMED column of Table 9.1; otherwise we filled the NOT PERFORMED column. We use the SEMI-PERFORMED column to indicate which process areas were only partially performed. That is, we have evidence to conclude that the 3TU.Federatie brings directly, or indirectly, only part of the results that are expected in such process areas. For example, the IS requirements management (IsRM) process area (see Chapter 8): the 3TU.Federatie (i) obtains an understanding of IS requirements based on its target IS architecture - SG1, (ii) assures commitment to requirements from all the three universities - SG2, and (iii) defines specific policies for requirements management - SG3. However, the 3TU.Federatie does not use a database to manage requirements - SG4. Not all goals in the IsRM process area are satisfied and, therefore, we indicate in Table 9.1 that this process area is only partially performed.

The responses of the interviewees and the documents provided evidence to support that the 3TU.Federatie has maturity level 1 in the domains of partnering structure and coordination, and maturity level 2 in the domains of IS architecture and process architecture. Most of the process areas required for achieving maturity level 3 seemed quite extensive at first sight in the 3TU.Federatie. However, it was discovered that part of them are either not considered formally as a process, or simply what is done is not enough to satisfy all the needs of the goals to achieve in each of them. 
Table 9.1: Pilot assessment results: The 3TU.Federatie B-ITa maturity.

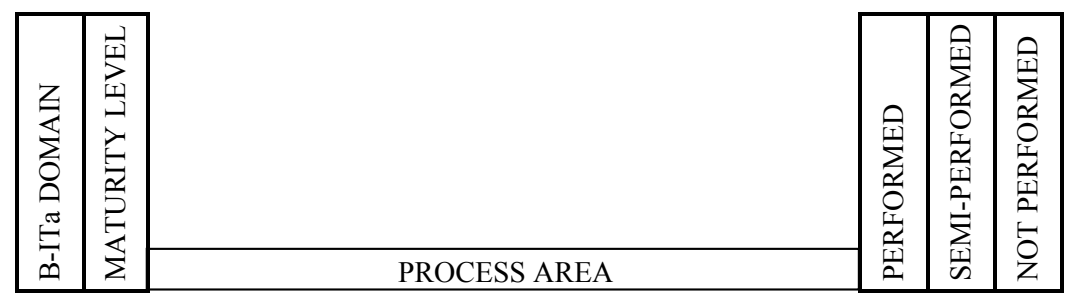

\begin{tabular}{|c|c|l|c|c|c|c|}
\hline \multirow{5}{*}{$\approx$} & 5 & & MRE & & & $\checkmark$ \\
\hline & 4 & Metric-based roles exploration & GSC & & $\checkmark$ & \\
\hline & 3 & Governance structure and compliance & SLA & & $\checkmark$ & \\
\hline & & Service level agreements definition & BMD & & $\checkmark$ & \\
\hline & 2 & Business model definition & & & & \\
\hline & & & & & & \\
\hline
\end{tabular}

\begin{tabular}{|c|c|l|c|c|c|c|}
\hline 5 & Inter-organizational process optimization & IoPO & & & $\checkmark$ \\
\hline & & Causal analysis and resolution & CAR & & & $\checkmark$ \\
\hline & 4 & Organizational process performance & OPP & & & $\checkmark$ \\
\hline & & EFC & & & $\checkmark$ \\
\hline & Event logs formal consistency & $\begin{array}{l}\text { Organizational process focus planning } \\
\text { Target process architecture formulation }\end{array}$ & PFP & & & $\checkmark$ \\
\hline & & TPA & & $\checkmark$ & \\
\hline & & Current process architecture description & CPD & $\checkmark$ & & \\
\hline 1 & & & & & \\
\hline
\end{tabular}

\begin{tabular}{|c|c|l|c|c|c|c|}
\hline \multirow{5}{*}{5} & \multirow{2}{*}{$\begin{array}{l}\text { Inter-organizational IS optimization } \\
\text { Risk analysis and mitigation }\end{array}$} & IoAO & & & $\checkmark$ \\
\hline & 4 & Quantitative IS portfolio management & & & $\checkmark$ \\
\hline & 3 & $\begin{array}{l}\text { IS requirements management } \\
\text { IS capabilities definition } \\
\text { IS portfolio management }\end{array}$ & & & $\checkmark$ \\
\hline & 2 & IsRM & & $\checkmark$ & \\
\hline & IsCD & & $\checkmark$ & \\
\hline & & IsPM & $\checkmark$ & & \\
\hline
\end{tabular}

\begin{tabular}{|c|c|l|c|c|c|c|}
\hline \multirow{6}{*}{} & 5 & & \multicolumn{3}{l|}{} \\
\hline & 4 & Quantitative coordination relation analysis & QRA & & & $\checkmark$ \\
\hline & 3 & Standardization & STD & $\checkmark$ & & \\
\hline & & Communication-oriented coordination & COC & $\checkmark$ & & \\
\hline & 2 & Informal communication adjustment & InCA & & $\checkmark$ & \\
\hline & & DTS & & $\checkmark$ & \\
\hline & 1 & & & & & \\
\hline
\end{tabular}




\section{CHAPTER 9. PILOT ASSESSMENT}

In the following, we present the perceptual $(\mathbf{P})$ and/or factual $(\mathbf{F})$ evidence we found for the SGs included in the four B-ITa domains. When a SG is not succeeded by an evidence explanation but by another SG, then the next evidence explanation belongs to both SGs (see for example CSA or IsCD in Section 9.4.2). We present only evidence for the process areas included in level 2 and level 3.

\subsubsection{Partnering structure}

\section{Business model definition (BMD) - SEMI-PERFORMED}

SG1 Define the CNO overall identity.

F The mission, vision, offered products/services and the competitive advantage are publically available in the 3TU.Federatie website and in several online publications where the 3TU.Federatie has been presented.

F One interviewee described how financial resources are managed in the CNO: "investments for the 3TU.Federatie are considered in the year budgets of each IT department of each university. This turns in a smooth financial decision making process that does not always need the approval of the Executive Committee for using part of the budget of the entire 3TU.Federatie".

$\mathbf{P}$ Two of the eight interviewees described how, according to them, the internal assets are distributed and used in the CNO. We got explanations about how the human capital of professionals working in the 3TU.Federatie is leveraged to contribute to the 3TU.Federatie realization. That is, how the skills and knowledge of the professionals are used to achieve goals. One of these interviewees expressed: "we have a strong commitment to have a well-skilled workforce in the 3TU.Federatie. I am not sure if the fact that we work in an educational environment is the cause of our curiosity for knowledge to improve our work. Raising the skills levels locally is important to us. By acquiring new knowledge and improving skills we help ourselves to unlock potential to face problems".

$\mathbf{P}$ As we got aware of the 3TU.Federatie SURFgroup, we could identify document transfer agreements which the three universities implicitly used when proposals, activities' results and research materials are exchanged between research institutions. For example, documents produced in the 3TU.Graduate School cannot be accessed by other committees.

SG2 Identify the external environment.

F The target market and the competitors are well known by all eight interviewees. The 3TU.Federatie does not perform a formal competitive 


\subsection{RESULTS AND RECOMMENDATIONS}

analysis. However, all know that in order to gain presence as universities of technology in the world, they need to work together to be at the level of top universities. One interviewee explicitly mentioned: "such external environment issues, you mention, were presented and discussed in the initial meetings we had as 3TU.Federatie. Since then, I believe all professionals in this CNO are aware of the environment where we are working".

SG3 Describe a multi-value configuration.

$\mathbf{P}$ No document was found presenting aspects related to investments, costs, and/or revenue. We suppose these documents must exist but at a level different from the level we had access to. We think they may exist at executive level of the 3TU.Federatie. This could be the reason for the (almost) consistent answer we got: "I'm not aware of the existence of documents containing such aspects. They could be there, but I have not heard about it".

SG4 Formulate an overall CNO plan.

F In the 3TU.Federatie SURFgroup, there are several documents presenting the plan of the CNO for both long and short term.

F Workgroups from different committees are preparing their roadmaps for the next six months. An interviewee who is involved in the Architecture Workgroup of the 3TU.Graduate School handed to us a draft of their roadmap.

Governance structure and compliance (GSC) - SEMI-PERFORMED

SG1 Describe the overall organizational structure.

F The 3TU.Federatie website presents a description of its main bodies and their related responsibilities. However, those descriptions are all we could find with respect to roles and responsibilities. Roles and responsibilities of specific workgroups, for example, are not formally described. Knowledge of roles and responsibilities at this level of granularity is only tacit.

$\mathbf{P}$ Everybody knows his/her own role within his own workgroup. Everybody is aware of the rights that each professional has on specific processes and ISs in each of the universities.

SG2 Define the inter-organizational policies.

F There is no formulation of business rules or decision-making procedures at $\mathrm{CNO}$ level. When interviewees gave explanations regarding policies and principles, they referred to the architecture principles they have defined for the Architecture Workgroup of the 3TU.Graduate School. We had access to the documents where they defined these principles. 


\section{CHAPTER 9. PILOT ASSESSMENT}

SG3 Define the CNO directions using the overall CNO plan as basis.

F The 3TU.Federatie directions are revised each six months in the general meetings they have for sharing knowledge and for planning activities. Deliverables prepared in such meetings are "flight plans" presenting the ambitions and projects for each workgroup in the committees. The term "flight plans" is used in the 3TU.Federatie to refer to documents that present a detailed description of the path to follow regarding projects in order to become fully operational. "The layout of the general meetings is (i) present results to all participants, (ii) plan future activities and define goals, and (iii) present plans to all participants".

F There was (almost) a consensus regarding where the plans and programs are defined: "general program priorities are discussed and established in the general meetings. Workgroup-specific programs are discussed and established in the meetings that each committee holds".

SG4 Ensure programs compliance.

F There are no formal activities performed for ensuring that the programs undertaken agree with policies and expectations.

$\mathbf{P}$ One interviewee commented: "Programs compliance is not explicit. However, I believe that the 3TU.Federatie general meetings help to control such a compliance... and at more operational level, the chairpersons of each workgroup also help to assure that what we do is what has been planned".

Service level agreements definition (SLA) - SEMI-PERFORMED

SG1 Negotiate the agreements.

$\mathbf{P}$ The definition of commitments to projects and the negotiation of agreements among the three universities are implicitly done during their work, although there is little encouragement for doing both practices in a formal way.

F There is an agreement upon description of issues that can be used as performance thresholds. For example, we had access to a functional services catalog that is used to direct the work of each university toward providing such services. "This services catalog will assure the quality of the products/services offered to the students in the three universities".

SG2 Describe SLA control issues.

F There is no description of escalation procedures. An interviewee mentioned: "there is no possibility for falling short of the expectations and 


\subsection{RESULTS AND RECOMMENDATIONS}

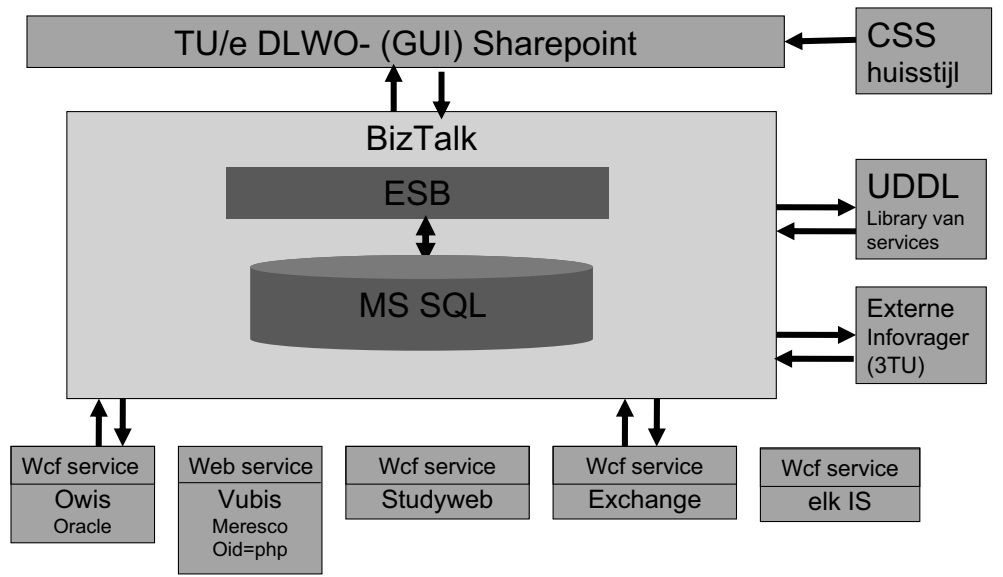

Figure 9.3: IS architecture of the Eindhoven University of Technology [87].

agreements. We, as university, must meet the expectations and agreements if we want to succeed as 3TU.Federatie. Everybody is committed. Escalation procedures and penalties/incentive policies do not apply in this CNO".

\subsubsection{IS architecture}

\section{Current IS architecture description (CSA) - PERFORMED}

SG1 Characterize the existing software applications.

SG2 Build the software application model.

F Individually, each university has described its current IS landscape to have all software applications at hand when defining the target IS architecture (see Figure 9.3 for an example of the IS architecture of the Eindhoven University of Technology). The CSA at CNO level is not required because no IS is collaborative in the 3TU.Federatie. "We will be connected by ESBs; we will not build IOS"'.

SG3 Define the IS standards and principles.

F From two interviewees, we got documents presenting the standards and principles for architecture. These documents were evidence for the existance of standards and principles that direct decision making regarding the IS architecture in the 3TU.Federatie. The principles are high-level judgements concerning ISs. They are linked with the objectives, values and culture of the 3TU.Federatie.

IS portfolio management (IsPM) - PERFORMED 
SG1 Define the target IS architecture.

F The target IS architecture has continuously been one of the most important issues to define in the 3TU.Federatie. One interviewee commented: "because the 3TU.Federatie operations completely depend on technology, define what is the target architecture is the main current task of the Architecture Workgroup of the 3TU.Graduate School".

F All interviewees were aware of the target architecture, and six of them have even depicted it at interview time during their explanations for our better understanding.

$\mathbf{P}$ Although there is no agreement to formally validate and verify the target IS architecture, four of the eight interviewees mentioned that this practice implicitly takes place during their meetings where the 3TU.Federatie target architecture is presented for discussion.

F The target IS architecture has been used as the basis for defining future investments in each of the three universities of technology.

SG2 Define insights and facts for decision making.

$\mathbf{P}$ When working in the definition of the IS target architecture, 3TU professionals have discussed issues as adaptability, flexibility, availability of ISs at university-level that are required for the collaborative work as 3TU.Federatie.

F There is no formal definition of key application metrics or IS risk management tasks at the level we had acces to. We believe that such activities occur at the executive level of the 3TU.Federatie. No interviewee was aware of this was indeed the case.

SG3 Develop a plan to obtain all participants' buy-in.

F Roadmaps and individual workgroups/committees plans are always presented in the general meetings that the 3TU.Federatie holds for sharing knowledge and for planning overall activities. This help to obtain commitment from the professionals of the three universities of technology.

\section{IS capabilities definition (IsCD) - SEMI-PERFORMED}

SG1 Analyze the IT and business skills of the participants.

SG2 Identify the motivating values.

SG3 Define the IS capabilities of the entire CNO considering the previous two SGs.

$\mathbf{P}$ The 3TU.Federatie culture encourages considering the skills and motivating values of the professionals as key enablers of collaboration. They are aware of the important of the IsCD. Implicitly, the definition 


\subsection{RESULTS AND RECOMMENDATIONS}

of capabilities is done because it is necessary for the work of the centers of competence and excellence and the committees. This definition of capabilities is not oriented to identify the IS skills of the entire CNO for using the ISs to achieve their goals. However, they know that they must efficiently use their ISs in order to offer world-class education in the new $\mathrm{CNO}$ environment they are creating.

IS requirements management (IsRM) - SEMI-PERFORMED

SG1 Obtain an understanding of IS requirements based on the target IS architecture of the CNO.

F In their explanations, five of the eight interviewees agreed on the considerable time they have spent in analyzing different scenarios for the target ISs status of the 3TU.Federatie. In the 3TU.Federatie SURFgroup, we had access to documents presenting such IS scenarios. Understanding, and defining, the most efficient way regarding ISs for the CNO took almost two years. In this period, all relevant IS requirements were agreed upon. The 3TU.Federatie is currently defining what specific ESB will help the most to achieve their goals.

SG2 Obtain commitment to requirements of all participating organizations.

F The two annual 3TU.Federatie general meetings are used for capturing the attention and commitment of all involved professionals to IS requirements. This is done by means of presentations and discussion sessions on what has been done, and what will be done.

SG3 Define policies for requirements management.

SG4 Use a database to manage requirements.

F The 3TU.Federatie does not define formal IS requirements management policies and it also does not use any requirements database to support the IS requirements management process.

\subsubsection{Process architecture}

\section{Current process architecture description (CPD) - PERFORMED}

Although the SG2 and SG3 in the CPD process area are not achieved (see below), we decided to consider that this process area has been performed. The presence of its main expected result (see Chapter 8) in each of the three universities of technology has been the rational behind our decision.

SG1 Characterize the existing processes. 
F All eight interviewees agreed on that the 3TU.Federatie has no collaborative processes. The service provisioning processes are universityspecific and together they form the current process architecture of the 3TU.Federatie as a whole. Each university has its own processes and they are formally defined and documented.

SG2 Establish the CNO's measurement repository.

SG3 Establish the CNO's process asset library.

F The 3TU.Federatie does not have an established formal measurement repository neither a process asset library.

F All interviewees explained that in the 3TU.Federatie there are no collaborative processes. One interviewee even added: "let me explain you one reason for the lack of collaborative processes. Don't forget that we are a networked organization working as federation. Think in the United States of America or in Germany. They are also federations. They are unions of partially self-governing states or regions united by a central entity. The three universities in the 3TU.Federatie work together for achieving common goals; however, each university has the freedom to choose how to offer its services. We keep variety in terms of service-provisioning processes". In addition, all interviewees considered that the absence of collaborative processes diminishes the necessity for maintaining a measurement repository and a process asset library at CNO level.

Target process architecture formulation (TPA) - SEMI-PERFORMED

SG1 Establish general tailoring guidelines.

F To describe their process architecture, the 3TU.Federatie uses the enterprise architecture method of BiZZdesign ${ }^{2}$. Furthermore, within the 3TU.Federatie, professionals have decided to use open standards wherever possible (if this is not possible, they give preference to de facto standards). The modeling language $\mathrm{ArchiMate}^{3}$, has been selected to model their enterprise architecture which also covers the process architecture.

SG2 Identify external interfaces.

$\mathbf{P}$ It seems that these interfaces have not been formally identified. However, four of the interviewees explicitly mentioned that the extended

\footnotetext{
${ }^{2}$ BiZZdesign (http://www.bizzdesign.nl/) is a spin-off company of the Telematica Instituut (currently called Novay) on the basis of the Testbed research project. This project was conducted in the period 1996-2001 by theTelematica Instituut, ABP, the Dutch Tax Department, IBM, and ING. Its aim was to deliver a design approach and modeling environment for business processes.

${ }^{3}$ For detailed information on ArchiMate, please see http://www.archimate.org/
} 


\subsection{RESULTS AND RECOMMENDATIONS}

goal of the 3TU.Federatie, is to create a work environment to which other universities, both from the Netherlands and from other countries, can easily hook up for collaborative work (see Figure 9.1).

SG3 Gather information about product/service offerings.

SG4 Build the target process model (graphical description).

F Several documents found in the 3TU SURFgroup present expected results for these two SGs. The most representative document is the one entitled "3TU-Op weg naar een federatieve Digitale Leer- en Werkomgeving" (in English: "3TU-On the road to a federative elearning/e-science environment"). This document was referred to as the "BiZZdesign report" by the interviewees when they gave explanations regarding the process architecture of the 3TU.Federatie. The document includes the graphical description of general processes that will be support the service provisioning. Triggers, actors and used information also are specified. For the definition of this target process architecture different roles were considered. They started from the perspective of the students and afterwards they considered the perspective of the professors and other people concerned at education.

SG5 Define the most important activities to support the CNO product/service offerings.

F Although the "BiZZdesign report" explicitly states that it only includes the most relevant educational processes, no interviewee confirmed that it indeed the case was. The interviewees only mentioned that the 80 services described in that report were the most essential ones. It must be noted that processes and services are not the same. Interviewees did not express that the "BiZZdesign report" includes the descriptions of critical processes.

\section{Organizational process focus planning (PFP) - NOT PERFORMED}

SG1 Determine process improvement opportunities.

SG2 Plan and implement process improvements.

SG3 Deploy CNO process assets and incorporate lessons learnt.

F No process improvements are selected, planed and/or implemented. No organizational process assets are deployed. This is a result of having a not fully operational 3TU.Federatie. Improvement of processes comes in later stages of operation. 


\subsubsection{Coordination}

\section{Direct supervision (DTS) - SEMI-PERFORMED}

SG1 Plan the supervision.

F The work within the 3TU.Federatie is supervised. However, there are no formal planning activities for doing so. Each workgroup has its own chairperson who supervises the work and leads on appropriate issues at meetings. Three of the interviewees were chairpersons of different workgroups and we could confirm that no time is used to explicitly select a supervisory strategy and/or model.

SG2 Establish a safe and trust relation with the supervisees.

$\mathbf{P}$ The chairpersons (supervisors) and the workgroup members (supervisees) maintain a good work relation. Because everybody is committed for achieving the 3TU.Federatie goals through making this CNO fully operational, there is a work environment where trust drives behavior. One of the interviewees commented: "I believe our chairpersons primarily have two responsibilities. First, they have a supervising/supporting role, they are developers of people. However, they also have professional work that they do. Everybody has its own tasks. We have a good organization. We do not do our own thing but we also do not take strict orders from chairpersons. From my point of view, supervision is well-done in the 3TU.Graduate School, and I believe that this is the case for the entire 3TU.Federatie".

SG3 Monitor the ongoing activities and provide instructions to adjust problems.

F Monitoring of activities takes place in the meetings of each workgroup and in the 3TU.Federatie general meetings (every six months). The chairpersons of the different groups are always present in the meetings of a particular workgroup. This helps to monitor activities from a 3TU.Federatie perspective and not only from the perspective of a single workgroup. A meeting generally starts with presentations concerning the work that has been done. Then, discussions take place for future planning considering informal adjustment instructions that can come from each attendee. Finally, future plans are presented.

SG4 Build a learning agenda.

F Because supervision is not formally planned, learning agendas for supervision improvements are not developed.

Informal communication adjustment (InCA) - SEMI-PERFORMED

SG1 Identify the social composition of the CNO. 


\subsection{RESULTS AND RECOMMENDATIONS}

SG2 Influence the development of the CNO informal structure.

F None of the SGs included in this process area is achieved by performing structured practices. However, informal communication does take place for adjusting work among the three universities. One interviewee stated: "Because the 3TU.Federatie meetings are held in the middle of the country, we always use our train travel time to discuss 3TU.Federatie issues that commonly are later posed for discussing in the meetings".

F Informal communication was recognized by all interviewees as a valuable aspect for coordination.

$\mathbf{P}$ Professionals find easy to share ideas and to freely discuss 3TU.Federatie issues on informal settings. "Sometimes, in the coffee breaks, I just chat with my 3TU.Federatie colleagues. This chat is not like the discussions in the meeting. We discuss more how we feel about the issues and concerns presented in the meeting. I really like that most of us are active participants in the meeting, which help to informally chat during our coffee breaks".

\section{Communication-oriented coordination (COC) - PERFORMED}

SG1 Agree on communication channels and common language.

F The main communication channels used in the 3TU.Federatie are email messages, face-to-face meetings and the virtual collaboration environment 3TU SURFgroup.

F All interviewees were aware of the importance of communication channels agreements to ensure complete communications and to sustain the collaboration.

SG2 Create a shared knowledge considering inside/outside information.

$\mathbf{P}$ This SG is implicitly achieved in the meetings that this CNO holds. Meetings are the only place where participants can personally, and directly, build and develop their relationship as a cohesive group and where they can share knowledge for creating a common understanding regarding the results of each of the workgroups.

SG3 Use IT to enable the communication between the participants.

F Besides the e-mail, the 3TU SURFgroup is the IT platform used by all participants for collaboration and communication.

SG4 Ensure that participants use continuous learning to respond to immediate needs.

F Outside the meetings, continuous learning is supported using the $3 \mathrm{TU}$ SURFgroup. 
CHAPTER 9. PILOT ASSESSMENT

\author{
Standardization (STD) - PERFORMED
}

SG1 Standardize processes.

SG2 Standardize outputs.

F The 3TU.Federatie has no collaborative processes. Service provisioning processes can differ from one university of technology to another. However, the standardization of the services to offer (outputs) was one of the most important issues that the interviewees discussed during the interview. This standardization has been documented in the "BiZZdesign report".

SG3 Standardize skills/competences.

F Training/instruction is available to familiarize 3TU.Federatie professionals of workgroups where work results are considerable tool-based, with different methods and tools. For example, the professionals involved in the Architecture Workgroup of the 3TU.Graduate School have received training on ArchiMate.

\title{
9.4.5 Recommendations
}

After conducting the pilot assessment, we were able to define a set of practices that we recommend for the 3TU.Federatie in order to come up with ideal settings for improving B-ITa as a CNO. These practices are derived from the set of SGs to achieve in each of the process areas included in the ICoNOs MM (see Chapter 8). We only included recommendations that can help the 3TU.Federatie to reach level 3 of maturity because the process areas of higher levels are more effective when the collaboration is already fully operational. That is, the recommendations presented below correspond to the B-ITa process areas (at level 2 and 3) that were NOT PERFORMED or SEMI-PERFORMED in the 3TU.Federatie (see Table 9.1). The order used to list the recommendations does not imply prioritization. The first three recommendations correspond to the partnering structure domain; the next two recommendations (i.e., 4 and 5) are related to the IS architecture domain; recommendations 6 and 7 correspond to the process architecture domain; and the last recommendation gives advice on the coordination domain.

1. Describe the multi-value configuration of the 3TU.Federatie defining the cost structure, and the value and revenue models. This helps to state the revenue potential of the 3 TU.Federatie and its economic feasibility in order to make decisions regarding costs reduction or required increased investments for committees, or for particular projects.

2. Define agreement control issues like escalation procedures in order to set up steps that should be taken when specific services or results do not meet the agreements made between the three universities. These control issues must be institutionalized in the 3TU.Federatie in order to warn it of any problem so that (immediate) actions can be taken. 


\subsection{RESULTS AND RECOMMENDATIONS}

3. Establish a governance model in order to formally structure project priorities and allocate resources and decision rights as well as to create accountability, and to assure that changes are achieved on the basis projects at $\mathrm{CNO}$ level and at the level of individual partners. Governance helps to understand who is responsible for what in which specific way.

The rational for these first three recommendations can be traced back in the results of the BMD, SLA and GSC process areas in Section 9.4.1. That is, (i) all interviewees were not aware of the existence of documents presenting aspects related to investments, costs, and/or revenue. In the SURFgroup, we could not find documents containing such aspects; (ii) the fact that everybody in the 3TU.Federatie is committed to meet the agreements among the three universities diminishes the necessity of escalation procedures. However, commitment can not guarantee performance; therefore, escalation procedures are important to define the steps to take when agreements are not met; and (iii) one of the interviewees presented to us a document describing a complete governance model that was intended to be implemented in the 3TU.Federatie. We believe that its implementation is important in order to achieve the SGs included in the GSC process area.

4. Define the IS capabilities of the 3TU.Federatie in order to be aware of its ability to use information systems for both achieving congruence with the educational environment where it works and improving its operational performance. IS capabilities involve competencies and routines that complement information systems.

5. Consider introducing a requirements database (or a commercial requirements engineering tool) in support of the process of managing the IS requirements. This database stores the requirements as entries (rather than maintaining requirements in text documents only). This helps to trace IS requirements from specification to their actual implementation.

These two recommendations come from the results presented in the IsCD and IsRM process areas in Section 9.4.2. That is, (i) it seems that the 3TU.Federatie considers the skills and motivating values of all the professionals as key enablers of collaboration. However, definition of IS capabilities is not a formal practice in this CNO; and (ii) although the 3TU.Federatie has spent considerable time to understand and to define its IS requirements, it does not formally define management policies over such requirements and it does not use a requirements database in support of requirements management.

6. Operationalize the general process/data architecture described in the functional services report into a single-organization level. That is, once the 3TU.Federatie has defined the functional services and the processes/data that support them, the next step is to use the service/process/data definitions in the description of each university's process/data architecture (e.g., as TU/e does this). 
7. Plan, implement and deploy process improvements based on a thorough understanding of the 3TU.Federatie strengths and weaknesses of both the processes required to support the functional services, and the organizational process assets. These process assets represent investments that are expected to provide current and future value to the three universities.

The rational for recommendations 6 and 7 can be traced back in the results of the TPA and PFP process areas in Section 9.4.3. That is, (i) the 3TU.Federatie has already built a process model to support its service provisioning: the "BiZZdesign report". Services should be delivered equally well by the three universities; however, the service delivery process can be established at university-level. Therefore, in order to be fully operational, each university must use the functional services presented in the "BiZZdesign report" to operationalize its process architecture; and (ii) because not having a fully operational 3TU.Federatie, no process improvements are selected, planned and/or implemented.

8. Adjust and control the work between the three universities by informal communication outside the "imposed" structural constrains for day-to-day operations. That is, to define the social composition of the 3TU.Federatie, to identify the relationship-based structures, to set informal communication channels, and to promote extracurricular joint activities.

Finally, this last recommendations comes from the results presented in the InCA process area in Section 9.4.4. That is, although informal communication does help to adjust the work among the three universities, none of the SGs included in the InCA process area is achieved by performing formal structured practices.

It must be noted that we did not derive recommendations from the results of the DTS process area (even though this process area resulted to be SEMIPERFORMED). We believe that the way of supervising in the 3TU.Federatie is linked to the situation at hand and it works properly in their settings. Supervisors come direct to the point concerning what they see as strong or week issues in the projects. Interviewees were positive with respect to the direct supervision that takes place in the 3TU.Federatie.

\subsection{Usability of the ICoNOs MM}

The ICoNOs MM helped us to assess the B-ITa maturity of the four different domains if the 3TU.Federatie and to define a list of recommendations based on the process areas, SGs and SPs that the model includes. This situation makes us to conclude that the ICoNOs MM is indeed useful for assessing B-ITa in CNOs (what satisfies the improvement goals presented in Chapter 4). In order to support this conclusion, we were interested in the perceptions of the 3TU.Federatie professionals who were interviewed with respect to the ICoNOs MM. After conducting the pilot assessment, we presented the results of the assessment (see Table 9.1) and 


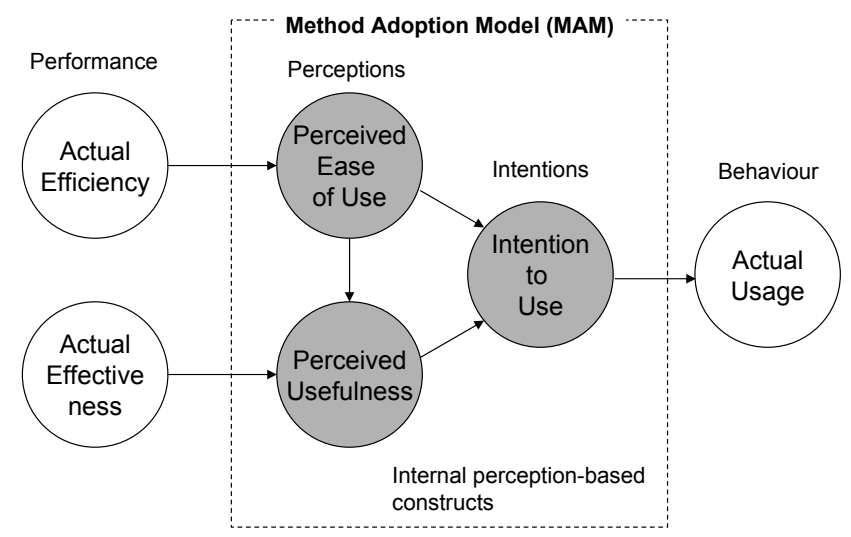

Figure 9.4: The Method Evaluation Model (adapted from [209]).

the list of recommendations introduced in the previous section to the interviewees. Our goal was to know if they considered these recommendations convincing and helpful, and if they accept the ICoNOs MM as well. This helps evaluate the usability of the results obtained after using the ICoNOs MM for assessing B-ITa in a real-life CNO. Using a post-task survey, we collected interviewees' perceptions on the assessment process, the model, and the results. In the next sections, we present the perception-based approach we used for evaluating the usability of our model (Section 9.5.1), and the results of such evaluation (Section 9.5.2).

\subsubsection{Perception-based evaluation approach}

Our perception-based evaluation approach is based on the Method Evaluation Model (MEM) created by Moody [209] for evaluating IS design methods. The core of the MEM, called the Method Adoption Model (MAM) - see Figure 9.4, is what we used in our evaluation. MAM consists of the same perception-based constructs as the Technology Acceptance Model (TAM) [77]. The work of Condori Fernández and Pastor [216], Abrahão and Poels [1], and Téllez [299] are empirical studies that present MAM in practice. The TAM has also been used by a considerable number of empirical studies [177]. The perception-based constructs included in MAM are:

- Perceived Ease of Use (PE): the extent to which a person believes that using a specific method would be free of effort.

- Perceived Usefulness (PU): The extent to which a person believes that a specific method will be effective in achieving its objectives.

- Intention to Use (IU): the extent to which a person intends to use a specific method. 


\section{CHAPTER 9. PILOT ASSESSMENT}

The model recognizes that the PE (efficiency) and the PU (effectiveness) of a method play an important role to know if the method will be adopted into practice depending on the IU.

Our approach differs from previous studies that used MAM [1, 216, 299] in that it does not use statistics for analyzing the collected data. Because we were interested (i) in what the interviewees thought and on their justifications for such thoughts, and (ii) in identifying direct improvement opportunities for the ICoNOs MM from interviewees' perceptions, we did not design likert-scale questions but used open questions to collect such perceptions. Clearly, this prevented us to obtain quantitative data and to use of statistics for analyzing them. It must be noted that our goal was similar to previous studies that used MAM. That is, we wanted to know how people (in our case, the interviewees) perceive a specific model/method (in our case, the ICoNOs MM) regarding the three perceptionbased constructs (i.e., PE, PU and IU). However, our research was completely qualitative-oriented (see Chapter 3). Table 9.2 lists the open questions included in the post-task survey presenting which perception-based constructs they relate to.

Table 9.2: Post-task survey for the perception-based evaluation.

\begin{tabular}{|c|c|c|c|}
\hline Question & PE & PU & IU \\
\hline $\begin{array}{l}\text { 1. Do you consider all these recommendations convincing } \\
\text { and helpful? Why or why not? }\end{array}$ & & $\bar{x}$ & \\
\hline $\begin{array}{l}\text { 2. Do you think that you could achieve business-IT align- } \\
\text { ment without being aware of the results (presented in the } \\
\text { Table 9.1) and specifically without recommendations } 1,2 \text {, } \\
3 \text { and } 8 \text { )? Why or why not? }\end{array}$ & $x$ & $x$ & \\
\hline $\begin{array}{l}\text { 3. Are recommendations } 4,5,6 \text { and } 7 \text { useful for the } 3 \mathrm{TU} \\
\text { Federatie in its actual operational stage? Why or why } \\
\text { not? }\end{array}$ & $x$ & $x$ & \\
\hline $\begin{array}{l}\text { 4. The ICoNOs MM has (still) no tool for conducting as- } \\
\text { sessments. Therefore, we used interviews and documents } \\
\text { reviews that helped us to present qualitative results. Did } \\
\text { you find this assessment manner appropriated? Or would } \\
\text { you prefer a tool for obtaining quantitative results pro- } \\
\text { viding numerical descriptions rather than narrative rec- } \\
\text { ommendations? }\end{array}$ & $x$ & & \\
\hline $\begin{array}{l}\text { 5. Would you use and recommend to others the use of the } \\
\text { ICoNOs MM for this kind of assessment? }\end{array}$ & & & $\times$ \\
\hline
\end{tabular}

We sent the survey to the eight interviewees. However, only six interviewees answered the questionnaire. The answers of the interviewees to the questionnaire are presented in Appendix E (some of the answers are presented in Dutch). 


\subsection{USABILITY OF THE ICONOS MM}

\subsubsection{Evaluation results}

In this section we analyze the interviewees' answers to each of the three perceptionbased constructs used for this evaluation.

\subsubsection{Perceived ease of use}

This construct presents interviewees' perceptions about the ease of use (efficiency) of the ICoNOs MM in general. We aimed to identify whether interviewees thought that our model can reduce effort required to improve B-ITa in CNOs. Questions 2, 3 and 4 relate to $\mathrm{PE}$ (see Table 9.2).

\section{Question 2}

- 3 out of 6 respondents find the recommendations related to the partnering structure and the coordination domains (i.e., recommendations 1, 2, 3 and 8) useful and easy to use for making improvements towards B-ITa. According to them, these four recommendations can (i) contribute to a perfect and efficient operation and maintenance, (ii) drive proper B-ITa improvements, (iii) make the 3 TU.Federatie to have more control of alignment issues, and (iii) help to achieve the efficiency and results that the 3TU.Federatie expects.

- In contrast, one respondent believes that recommendations $1,2,3$ and 8 do not reduce effort for improving their B-ITa. He explicitly mentions that even without these four recommendations they could be able to achieve alignment in the 3TU.Federatie. A possible explanation could be that, based upon his answers, this interviewee perceives our recommendations as suggestions to counter situations that are not flaws in the 3TU.Federatie (from his particular point of view). He was expecting recommendations directly related to solve problems.

- Finally, the last 2 respondents were hesitant in giving a explicit answer to this question.

\section{Question 3}

- 3 out of 6 respondents think recommendations related to the IS architecture and the process architecture domains (i.e., recommendations 4, 5, 6 and 7) do not support effort reduction for improving B-ITa in the CNO. These respondents believe that such recommendations do not apply at the 3TU.Federatie level but at the university-level only. A possible explanation could be that no cross-organizational processes or ISs are planned to be implemented in the 3TU.Federatie (see Section 9.4).

- The other three respondents find, however, recommendations 4, 5, 6 and 7 useful. Even one of them explicitly mention that they are currently working on issues related to recommendations 4,6 and 7 . 


\section{Question 4}

- 4 out of 6 respondents find the use of interviews and document analysis when applying the ICoNOs MM appropriate for assessing B-ITa in CNOs. The qualitative results we present to them were, in general, enough to understand the maturity of the 3TU.Federatie in each of the four B-ITa domains that our model includes. However, in their answers, it can be noted that respondents would like to obtain more information/explanations of the results and recommendations.

- In contrast, the other two respondents prefer a tool for obtaining quantitative results providing statistical results and numerical descriptions rather than narrative recommendations. They believe that quantitative results are more accurate and can avoid having only general recommendations.

Most of the answers related to PE were positive. Those interviewees' perceptions that were not positive helped us to identify one improvement opportunity: the recommendations derived from the ICoNOs MM assessments should not be briefly described; they should include ample explanations in order to be more understandable in context; examples of specific terms can help to enrich the recommendations.

\subsubsection{Perceived usefulness}

This construct presents interviewees' perceptions about the usefulness (effectiveness) of the ICoNOs MM. We wanted to identify whether our model can indeed improve B-ITa in a CNO according to the point of view of the interviewees. Questions 1, 2, and 3 relate to PU (see Table 9.2). Because we have already presented the results of questions 2 and 3 in the previous paragraphs, we only show the answers to question 1 in the following.

\section{Question 1}

- 4 out of 6 respondents consider our recommendations in the main convincing and helpful for improving B-ITa in the 3TU.Federatie. However, the answers of three of these respondents emphasize the general and technical manner in which we formulate and present the recommendations. They expected operational guidance and specific advise, and more easy-to-read explanations.

- 1 respondent took a neutral position; he made no clear commitment to give a positive or negative answer to this question. He stated: "at reading time, your recommendations seem very useful considering that your model is the first model that can give this kind of recommendations to improve alignment issues in network organizations". However, he turned this positive comment into a neutral answer by saying that he would like to apply and discuss the recommendations before giving a more concrete answer to the question. 


\subsection{USABILITY OF THE ICONOS MM}

- the last respondent is hesitant towards the usefulness of the recommendations for improving B-ITa, and towards the ICoNOs MM itself. From his answer, it seems that he expected much more information about the ICoNOs MM and about the manner we analyzed all interviewees' answers to define what B-ITa process areas were, or were not, performed in the 3TU.Federatie. To put it briefly, he cannot trust the assessment procedure without having more information about the model, the procedure itself, and the way we analyzed the data collected for the assessment.

\subsubsection{Intention to use}

Finally, the IU construct presents interviewees' intentions to use the ICoNOs MM and to recommend to others its use for B-ITa assessments in CNOs. Question 5 relates to IU (see Table 9.2).

\section{Question 5}

- 2 out of 6 respondents would not (yet) use or recommend the use of the ICoNOs MM. Their reasons are: (i) the model must first be used in more pilot assessments to assure its validity, and (ii) the model must be presented in a "management-proof" way meaning that each person, independently of his/her background, can read and understand the recommendations easily.

- Other two out of 6 respondents hesitate in their answers. One of these respondents answered: "Maybe, because the basis is OK". However, he would like to increase his understanding of our model and of the way we analyze the data collected for the assessment before recommend to others its use. The other respondent would recommend the ICoNOs MM only after using it in order to learn more about the model itself.

- 1 respondent considers that B-ITa assessments in CNOs are considerably important and he is confident to recommend to others the use of our model.

- The last respondent does not give an explicit answer to this question.

Based on the answers to question 5, we could identify a relatively low value given by the interviewees to the use of the ICoNOs MM and its recommendations. This low value can be traced back to our choice of the information included in the document used to present the results to them. We only provided a summary of the results in each B-ITa domain (see Table 9.1) and the list of recommendations presented above in this chapter. This represents a partial view of what we did. That is, the document included the results and recommendations but has not explanations about the manner we analyzed the data collected in the interviews, or about the ICoNOs MM. Their knowledge of the ICoNOs MM and of the pilot assessment we conducted in the 3TU.Federatie was limited to what we explained to them during the assessment. This could restrict the interviewees in that they did not realize the importance of using our model for improving B-ITa in CNO 
settings. We had to present to them a summary of the ICoNOs MM, and the complete list of results and recommendations in the detailed way that we used to present them in this chapter (see Section 9.4).

In addition, these answers also reflect that more empirical research is required to support the validity of the ICoNOs MM. Multiple pilot assessments using the ICoNOs MM can serve as supportive evidence for increasing the status of the model in practice. This, consequently, can have a positive impact in the acceptance and use of the ICoNOs MM.

\subsection{Limitations of the assessment}

Altought the results in the pilot assessment were clear with respect to the maturities of the B-ITa domains, the manner for mapping the data gathered into an overall maturity level is not well-defined. So far, we have done this in a perceptional/subjective way. Standardizing this methodology for assessing B-ITa maturity involves defining an acceptable method for determining maturity levels.

It is important to state that the interviewees were not systematically selected and, therefore, they were not necessarily representative. Participants selection was mostly purposive. Interviewees were identified by our contact person in the 3TU.Federatie to ensure the breadth and depth of knowledge necessary to address our interests on the four B-ITa domains and to provide diversity of perspectives about alignment. However, all eight interviewees were involved in the 3TU Graduate School, which can limit their view on the 3TU.Federatie as a whole.

The perceptions of the interviewees with respect to the efficiency, effectiveness and usability of the ICoNOs MM are reasonably positive. According to the point of view of the six interviewees who answered our post-task survey, our model scores moderately good. Dissenting comments from interviewees will drive improving our model for assuring its practical adoption. The main shortcoming of our model that we identified in the interviewees' perceptions is the lack of information to present the results and recommendations in an understandable fashion.

While reflecting on the perceptions of the interviewees and on perceptions in literature on MMs, we can conclude that the ICoNOs MM is in its initial stage of deployment. When the CMM started gaining acceptance, it was an ad hoc MM (http://www.sei.cmu.edu/cmm/). This created confusion concerning the scale of the CMM itself. Evidence can be found to claim that any MM faces the same situation. The ICoNOs MM is not an exception. This is a normal stage of development which can be addressed by conducting, in our case, a long-term study including multiple organizations that validates the maturity scale and the SGs and SPs associated in each B-ITa maturity level (see step DEPLOY in the MM development process we presented in Chapter 4).

\subsection{Summary}

In this chapter, we presented the pilot assessment we used to evaluate the usability of the ICoNOs MM in a real-life context. That is, we used the most up-to- 


\subsection{SUMMARY}

date version of our model to assess the B-ITa maturity of one specific CNO: the 3TU.Federatie.

After conducting the pilot assessment, we presented (i) the results of the assessment and (ii) a list of recommendations for improving B-ITa in the assessed $\mathrm{CNO}$, to the interviewees. Using a perception-based evaluation approach, we aimed to know how they considered those recommendations, the ICoNOs MM, and the assessment procedure itself. This helped us to evaluate the efficiency, effectiveness and usability of our model from the specific viewpoint of the interviewees.

The perception-based evaluation revealed the need of more empirical research and findings to gain higher acceptance of the ICoNOs MM. The perceptions of the interviewees with respect to our model are reasonably positive. One improvement opportunity was identified from such perceptions: the ICoNOs MM assessment results must include enough information in order to make the results and recommendations more understandable. 



\section{Part IV}

\section{Conclusion}





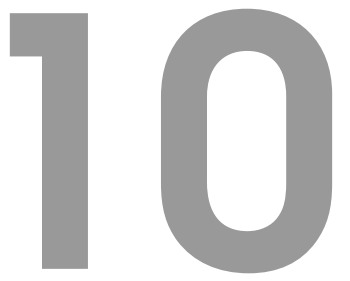

\section{Discussion and future work}

MMs seem a suitable vehicle for organizations to use to gain a deeper understanding of how they progress toward better alignment. MMs have been developed to assess specific areas against a norm. Based on maturity assessments, organizations know the extent to which activities in such areas are predictable. That is, organizations can be aware of whether a specific area is sufficiently refined and documented so that the activities in this area now have the potential to achieve their desired outcomes. Previous research [95, 189, 312] has recognized that BITa can be achieved at various levels of maturity. Although literature proposes MMs to assess B-ITa (see Chapter 4), there is no MM that specifically addresses the aspects needed for achieving alignment between business and IT in CNOs. In order to fill this gap, in this dissertation, we have introduced the ICoNOs MM. This MM allows assessing B-ITa in cross-organizational settings in order to plan and implement B-ITa improvements. Focus of the ICoNOs MM is on identifying which B-ITa practices are performed in a specific CNO working on a B-ITa project. The ICoNOs MM is the first solution which explicitly enables CNOs to systematically assess domains related to B-ITa interrelating facts and perceptions. Without our model, the maturity of B-ITa in CNOs remains practically unattended which makes it difficult for chief officers (CIOs and/or CEOs) to learn from CNO B-ITa projects for planning and implementing real improvements.

In Chapter 2, by discussing different issues related to the main three concepts of the research presented in this dissertation, we produced working definitions and understanding of the concepts of B-ITa (Section 2.1) and of a CNO (Section 2.2) for use throughout the dissertation. We defined B-ITa as being the process to make the services offered by IT support the requirements of the business - whether such services are offered individually by one participant in the CNO or collaboratively by the entire network - so that value is created for the participating organizations of the CNO; and a CNO as any "mix-and-match" network of profit-and-loss responsible organizational units, or of independent organizations, connected by IT, that work together to jointly accomplish tasks, reach common goals and serve customers over a period of time. In addition, we presented the advantages of using MMs (Section 2.3) and a brief description of the most well-known maturity model: the CMMI. 


\section{CHAPTER 10. DISCUSSION AND FUTURE WORK}

Our research has started with the analysis of current assessment approaches of B-ITa (see Chapter 4). We were interested in identifying and reusing the built-in design knowledge of such approaches so that we could avoid developing the ICoNOs MM from scratch. This made the development process cheaper and faster. Most importantly, it prevents future ICoNOs MM users from starting over when adopting the MM. During this initial phase of our research, we analyzed assessment approaches of B-ITa and MMs for CNOs. We have utilized results of this activity to identify major improvement goals and reusable knowledge to address and use in the development of the ICoNOs MM, respectively. We have developed our MM starting with the identification of B-ITa domains and B-ITa process areas, and finalizing with the description of goals and practices required in each process area. The identification of the B-ITa domains and the B-ITa process areas included in the ICoNOs MM was based on industry and practitioners experience, theories and models, and related literature (see Chapters 5, 6, and 7). To illustrate the detailed content of the ICoNOs MM, we have also presented the goals and practices from maturity levels 2 and 3 (see Chapter 8). Finally, we have validated the usability of our model based on a pilot assessment we conducted in a real-life CNO (see Chapter 9).

In this chapter, we first review the research questions presented in Chapter 1 (Section 10.1). Next, we present and discuss the main contributions of the dissertation (Section 10.2), the limitations of the ICoNOs MM (Section 10.3), and the future research issues that can contribute to further solve those limitations (Section 10.4).

\subsection{Reviewing the research questions}

The purpose of this research was to enable assessing cross-organizational B-ITa by developing the ICoNOs MM. In order to develop this MM, we started answering our first research question:

\section{Q1 What are the current assessment approaches of B-ITa?}

We reviewed current B-ITa MMs and we made a comparison of them in order to identify their advantages and shortcomings (see Chapter 4). This was useful to define the improvement goals and reusable knowledge to address and use in the development of the ICoNOs MM, respectively. Furthermore, we presented six MMs for CNOs. We wanted to identify what was important to include in our model to cover a cross-organizational perspective. In the following, we summarize this reusable knowledge and the improvement goals.

Reusable knowledge: our ICoNOs MM was expected to (i) be a development MM (similarly to BITAMA and COBIT), (ii) include not only domains but also specific process areas (similarly to MAAM and CMMI), (iii) have five maturity levels (similarly to CMMI in its staged representation), (iv) assess operational B-ITa (similarly to the CIO Council's assessment guide and 


\subsection{REVIEWING THE RESEARCH QUESTIONS}

MAAM), and (v) include the collaborative perspective in IT and process aspects (similarly to the MMs for CNOs we presented).

Improvement goals: our ICoNOs MM was expected to (i) be a continuous MM (similarly to one of the representations of CMMI), (ii) assess B-ITa in CNOs and not in single organizations, and (iii) include the collaborative perspective in organizational coordination and structure aspects.

Once we had defined the reusable knowledge and the improvement goals, we were able to address the second research question:

\section{Q2 How to develop an improved approach (in our case an MM)?}

Responding to our second research question, we used the MM development process presented in Section 4.2.1 of Chapter 4. Figure 10.1 presents an overview of the steps we followed to develop the ICoNOs MM. The shaded steps in the figure represent the steps that we have already conducted. This figure presents (i) the decisions made or the results obtained in different studies throughout the design of the model, and (ii) the chapters where we explain or present such decisions or results, respectively.

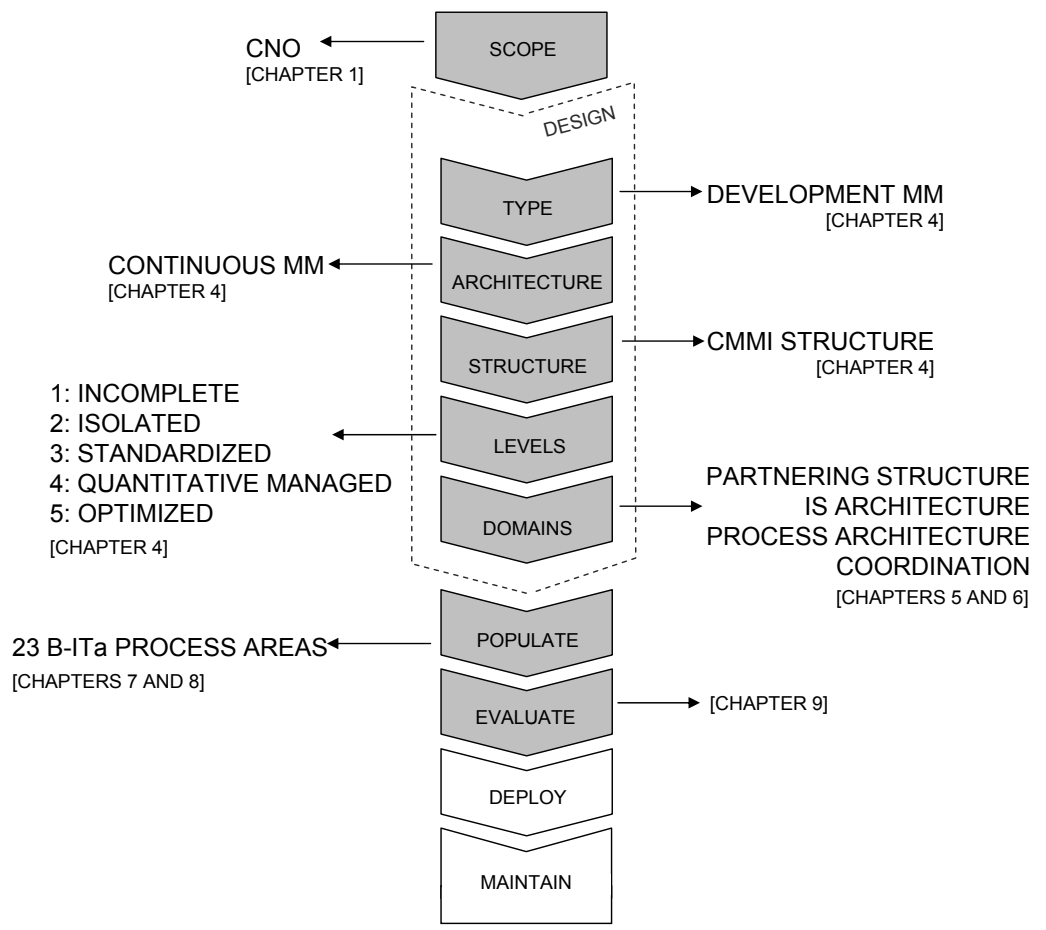

Figure 10.1: MM development process revisited (adapted from Figure 4.1). 


\section{CHAPTER 10. DISCUSSION AND FUTURE WORK}

First, in Chapter 1 we explained that the scope of our model covers CNO settings. That is, the ICoNOs MM can be applied to determine the maturity of the B-ITa of a whole networked organization and not of the B-ITa of each individual participating organization.

In Chapter 4, we furthermore described the main design decisions made for the ICoNOs MM, i.e., we established its type (development MM), architecture (continuous MM), structure (CMMI structure) and the levels it includes. In addition, in all of Part II of this dissertation, we illustrated how the design of an MM involves not only literature studies but also empirical studies. Case study research has constituted the main pillar when developing the ICoNOs MM. When conducting the case studies we have followed basic research designs and fundamental methods described by Yin [348], Eisenhardt [98], Kitchenham et al. [164], Paré and Elam [227] and Pawson and Tilley [230] (see Chapter 3).

In the development phase of the ICoNOs MM (see Figure 1.4), we first conducted a focus group session and a first case study to identify and to empirically validate the B-ITa domains (see Chapter 5). After analyzing the results of these two reseach methods, we were able to propose a new set of domains to be considered by CNOs to improve B-ITa: partnering structure, IS architecture, process architecture, coordination and cost management. However, in order to increase the validity of these domains, we conducted three new case studies in different CNOs running B-ITa projects (see Chapter 6). Only after conducting these studies, we had enough evidence to claim that

\section{partnering structure, IS architecture, process architecture and coordination}

were the B-ITa domains to address for improving alignment in CNOs. The next step was to identify the processes to include in each of the domains (see Chapter 7). We dealt with an exhaustive theoretical study for this purpose and, to confirm the identified processes, we conducted a new case study. Finally, we presented the final B-ITa process areas, specific goals and specific practices included in each B-ITa domain in Chapter 8. At this point, we wanted to answer:

\section{Q3 Is the improved approach valid and useful?}

In order to empirically confirm the utility of the ICoNOs MM, we have conducted a pilot assessment (case study) in a new CNO. The aim of this study was to use the ICoNOs MM to appraise the B-ITa maturity of the CNO investigated. After the assessment, we asked to professionals working in the appraised organization how they perceived the assessment process, the model, and the results (see Chapter 9). This helped us to identify how such professionals saw the ICoNOs MM in terms of its efficiency, effectiveness and usability.

The extensive research on all aspects of B-ITa in CNOs undertaken by all the theoretical and empirical studies mentioned in the previous paragraphs resulted in several original contributions. We present these contributions in the next section. 
10.2. CONTRIBUTIONS

\begin{tabular}{|c|c|c|c|c|}
\hline & \multicolumn{2}{|c|}{ PARTNERING STRUCTURE } & \multicolumn{2}{|l|}{ IS ARCHITECTURE } \\
\hline 5 & & & $\begin{array}{l}\text { Inter-organizational IS arch. optimization } \\
\text { Risk analysis and mitigation }\end{array}$ & $\begin{array}{l}\text { IOAO } \\
\text { RAM }\end{array}$ \\
\hline 4 & Metric-based roles exploration & MRE & Quantitative IS portfolio management & QPM \\
\hline 3 & $\begin{array}{l}\text { Governance structure and compliance } \\
\text { Service level agreements definition }\end{array}$ & $\begin{array}{l}\text { GSC } \\
\text { SLA }\end{array}$ & $\begin{array}{l}\text { IS requirements management } \\
\text { IS capabilities definition } \\
\text { IS portfolio management }\end{array}$ & $\begin{array}{l}\text { IsRM } \\
\text { IsCD } \\
\text { IsPM }\end{array}$ \\
\hline 2 & Business model definition & BMD & Current IS architecture description & CSA \\
\hline 1 & & & & \\
\hline
\end{tabular}

\begin{tabular}{|c|c|c|c|c|}
\hline & \multicolumn{2}{|l|}{ PROCESS ARCHITECTURE } & \multicolumn{2}{|l|}{ COORDINATION } \\
\hline 5 & $\begin{array}{l}\text { Inter-organizational process optimization } \\
\text { Causal analysis and resolution }\end{array}$ & $\begin{array}{l}\text { IOPO } \\
\text { CAR }\end{array}$ & & \\
\hline 4 & $\begin{array}{l}\text { Organizational process performance } \\
\text { Event logs formal consistency }\end{array}$ & $\begin{array}{l}\text { OPP } \\
\text { EFC }\end{array}$ & Quantitative coordination relation analysis & QRA \\
\hline 3 & $\begin{array}{l}\text { Organizational process focus planning } \\
\text { Target process architecture formulation }\end{array}$ & $\begin{array}{l}\text { PFP } \\
\text { TPA }\end{array}$ & $\begin{array}{l}\text { Standardization } \\
\text { Communication-oriented coordination }\end{array}$ & $\begin{array}{l}\text { STD } \\
\text { COC }\end{array}$ \\
\hline 2 & Current process architecture description & CPD & $\begin{array}{l}\text { Informal communication adjustment } \\
\text { Direct supervision }\end{array}$ & $\begin{array}{l}\text { InCA } \\
\text { DTS }\end{array}$ \\
\hline 1 & & & & \\
\hline
\end{tabular}

Figure 10.2: The ICoNOs MM at a glance (taken from Chapter 8).

\subsection{Contributions}

This dissertation makes a number of significant contributions in the area of B-ITa in CNOs. The contributions described in this dissertation can be summarized as follows:

CT1 our first contribution is the ICoNOs MM itself: an MM that can be used by CNOs to assess domains related to B-ITa initiatives which integrate multiple perspectives (see Figure 10.2 which is repeated from Chapter 8 for ease of reference).

CT2 our second contribution is a set of domains and processes that should be considered by CNOs in their efforts for improving B-ITa. We suggest that partnering structure, IS architecture, process architecture and coordination are these domains. We provide arguments explaining why networked organizations should consider the identified B-ITa domains. A new critical responsibility for CNOs involved in B-ITa projects should be weighing our empirically confirmed B-ITa domains in order to obtain new insights and to strive for B-ITa maturity.

CT3 we also introduced a systematic approach for the development of an MM (see Section 4.2.1 in Chapter 4). When developing the ICoNOs MM we realized that building MMs systematically is not a topic that is widely covered in the literature. Instead, most of the MM literature presents the resulting models only and does not discuss the model developing process 


\section{CHAPTER 10. DISCUSSION AND FUTURE WORK}

itself. The development of the ICoNOs MM consists of six general steps SCOPE, DESIGN, POPULATE, EVALUATE, DEPLOY and MAINTAIN that can assists MM developers when building new assessment approaches.

CT4 our fourth contribution is a set of eight B-ITa best practices for CNOs (see Chapter 7). The analysis of the information collected in the case study conducted to confirm the utility of the B-ITa process areas in a real-life CNO helped us to define those best practices.

CT5 the last contribution is that we provide evidence regarding B-ITa in CNOs collected in six case studies that other researchers can use to form hypotheses or set up new research designs. Several research streams within B-ITa can be identified (see Section 1.3 in Chapter 1). We have delimited our research to the description of B-ITa concepts and the identification of BITa mechanisms in CNO contexts (as presented in Table 1.1). We have found that partnering structure, IS architecture, process architecture and coordination are the B-ITa domains that CNOs must considered to improve alignment. The evidence collected in the studies conducted to identify such domains can be use for further investigation. For example, for studying causal relations among these four domains. This activity was out of the scope of our research.

These contributions and the research activities used to derive them have been progressively made public in several publications as presented in Table 10.1.

Table 10.1: Research activities, contributions and publications.

\begin{tabular}{|l|l|l|l|}
\cline { 3 - 4 } \multicolumn{2}{c|}{} & Activity & Publication \\
\hline \multirow{4}{*}{ CT1 } & Previous versions of the ICoNOs MM & {$[269,270,275]$} \\
\hline \multirow{4}{*}{ CT2 } & Initial attempts to identify domains & Focus group & \multirow{2}{*}[273]{} \\
\cline { 2 - 3 } & B-ITa domains validation & Case study & \\
\cline { 2 - 4 } & First version of the process areas & Literature survey & {$[275]$} \\
\cline { 2 - 4 } & B-ITa process areas validation & Case study & {$[277]$} \\
\hline CT3 & MM development process design & {$[271,275]$} \\
\hline CT4 & B-ITa best practices & Case study & {$[268]$} \\
\hline
\end{tabular}

\subsection{Limitations and open issues}

Although the ICoNOs MM has been designed using empirical research activities (see Chapters 5, 6 and 7), and that we have already testing its utility in practice (see Chapter 9), the model has still some limitations. In the following, we present several of these limitations and additional open issues. 


\subsection{LIMITATIONS AND OPEN ISSUES}

\subsubsection{ICoNOs MM shortcomings}

\subsubsection{Public vs. private CNOs}

We have mainly focused our research on cross-governmental partnerships (CGPs) instead of on partnerships involving profit-and-loss responsible independent businesses (private sector organizations). CGPs are networks of government agencies responsible to fulfill a shared public mission, connected by IT, that work together over a period of time. In a CGP, profit (exists but) is not relevant and loss consists of not fulfilling or overspending its public mission. We claim then that B-ITa in CGPs is important since it helps to improve organizational performance when providing services to citizens. However, we still have not enough evidence to extrapolate (with self-assurance) such a claim to private sector environments. Yet notwithstanding this lack of evidence, we do believe that there are similarities between organizations in the public and the private sector (see Section 6.6.3 in Chapter 6). We believe that partnerships in both sectors begin a B-ITa project with a solid mission statement that drives the strategic planning process to meet the common goal(s), and reminds the participating organizations of their work principles and respective roles in the network. As commonly the participants of private sector partnerships do, the participating government agencies of the studied CGPs (i) pool costs, skills, and core competences to provide world-class services that could not be provided by any of them individually; (ii) use ISs to respond dynamically to meet the ever-changing customer needs and to communicate and share information among them; and (iii) have a clear understanding of the common goal(s) and the functions of each of them in order to know what to expect from each other. Such characteristics are reflected in the CNO definition we present in Chapter 2. So, both sectors are similar. Only their purpose could vary. And, this difference in intention creates the environment in which they operate and how they do it.

\subsubsection{ICoNOs MM validation}

The next limitation refers to the validation of the ICoNOs MM as a whole (see Part III of this dissertation). Because of time constraints, we have conducted only one pilot assessment in a real-life CNO. Although this is enough to demonstrate the utility of the model, more experience using the ICoNOs MM could provide information to confirm the validity of the assumptions we make when using the model.

\subsubsection{Model assessment tool}

Commonly, MMs provide assessment tools to help organizations in their assessment efforts For example, the SCAMPI for conducting CMMI appraisals (http://www.sei.cmu.edu/cmmi/appraisals/) or the BITAMA online tool developed by de Koning and van der Marck (http://www.itzonderhoofdpijn.nl). The 


\section{CHAPTER 10. DISCUSSION AND FUTURE WORK}

ICoNOs MM has so far no assessment tool that CNOs can use to make selfassessments of their B-ITa. This lack of assessment tool causes that a B-ITa assessment process using the ICoNOs MM is only partly structured. In addition it relies in expert judgments and therefore the ICoNOs MM users could perceived the model as not that objective as the CMMI or the BITAMA are.

\subsubsection{Open issues}

Some interesting open matters remain to be addressed. For example, we acknowledge that despite the fact that the ICoNOs MM has been developed to assess B-ITa in cross-organizational environments, we are now interested to know what could happened when the ICoNOs MM is used to assess B-ITa in a non crossorganizational environment, i.e., a single organization. Also, to produce a complete MM we still need to complete the definition of goals and practices of the processes at levels 4 and 5 . The current version of the ICoNOs MM is elaborated until level 3 only (see Chapter 8).

The replication of the case study findings conducted to confirm the importance of the B-ITa domains in real-life settings (see Chapter 6) led us to consider the four B-ITa domains as the necessary domains to be addressed by CNOs when achieving B-ITa. The findings that were not replicated when making refinement of the initial CSMO configuration (see Figures 6.3, 6.5 and 6.7) are considered as additional domains. But, why is it valid to re-define B-ITa in a CNO in terms of only the four B-ITa domains? The ICoNOs MM can limit the vision of CNOs on their B-ITa, and they can leave additional B-ITa domains unattended what can mitigate the success of their B-ITa projects.

All these limitations and open issues can lead to plan a research agenda in order to counter them. In the next section, we list the future work derived from these limitations and new hypotheses that can be the basis for future research.

\subsection{Future work}

\subsubsection{Work based on the limitations and open issues}

In order to extrapolate results of the case studies to CNOs involving profit-andloss responsible organizations and not government agencies only, it is necessary to conduct case studies in such CNOs. We need to find more evidence from this kind of CNOs before generalizing results to other settings. Thereby, one has to separately investigate the B-ITa domains and processes in private sector CNOs. It would be useful if these studies involve small and medium enterprises. The CNOs studied can also be used to apply the ICoNOs MM, i.e., we could use the model to assess their B-ITa. This can increase the validity of the ICoNOs MM as a whole.

If the findings of the new case studies replicate new results, one should be able to consider new B-ITa domains as necessary domains instead of leaving them as 
additional ones. Results of new case studies can help to verify the final B-ITa domains included in the ICoNOs MM and, consequently, important additional domains would not remain unattended.

This dissertation presents the research activities we conducted through the ICoNOs MM development process (see Section 4.2.1 of Chapter 4) until the step EVALUATE (see Figure 10.1). In order to deploy the ICoNOs MM, we must (i) define the goals and practices related to the B-ITa processes at levels 4 and 5, and (ii) provide an assessment tool that CNOs can use to conduct B-ITa assessments by themselves. Therefore, (i) and (ii) are considered part of the immediate future work related to the ICoNOs MM.

\subsubsection{Work based on the B-ITa research framework}

In the introduction of this dissertation, we have presented that a research process for alignment studies covers two general themes: concept definition and concept validation. The scope of our research covered the three phases of the concept definition theme: description, mechanism and context, and only the description phase of the concept validation (see Table 1.1). Future work includes to verify the relations between the B-ITa domains and processes using more complex or statistical techniques, and to investigate and test the relation between B-ITa in CNOs and other topics in order to predict what could happen in future situations (mechanism and context phases of the concept validation theme, respectively).

\subsubsection{New hypotheses for a research agenda on B-ITa}

Based on the relations between the B-ITa domains in CNOs (see Chapter 6), a new claim derived from the study presented in this dissertation asserts that having a well-defined partnering structure as basis for the definition of the architecture of ISs and the processes architecture, helps to react promptly to business requirements. This is in a situation where coordination mechanisms are considered to manage the interaction and work among the participants in a networked organization. This claim is depicted in Figure 10.3 (which is adapted from Figure 6.8).

To derive formulations about the relations among the B-ITa domains, Figure 10.3 and the explanation of Figure 6.8 presented in Chapter 6 are used. We use a process theory perspective $[94,309]$ as instrumental in clarifying relations between the B-ITa domains for the purpose of providing explanation and understanding. Specific information concerning the maturity of each B-ITa domain only provides a necessary, but not sufficient, condition to understand a particular outcome. The common goal(s) of the CNO must be a reference point. Figure 10.3 presents both a sequence of relations (e.g. partnering structure and coordination precede IS architecture and process architecture - see timeline), and a dependency relation (see dotted line) between the B-ITa domains. These two kinds of relations structure the four B-ITa domains. These relations help to explain how CNOs achieve B-ITa by considering the four domains. 


\section{CHAPTER 10. DISCUSSION AND FUTURE WORK}

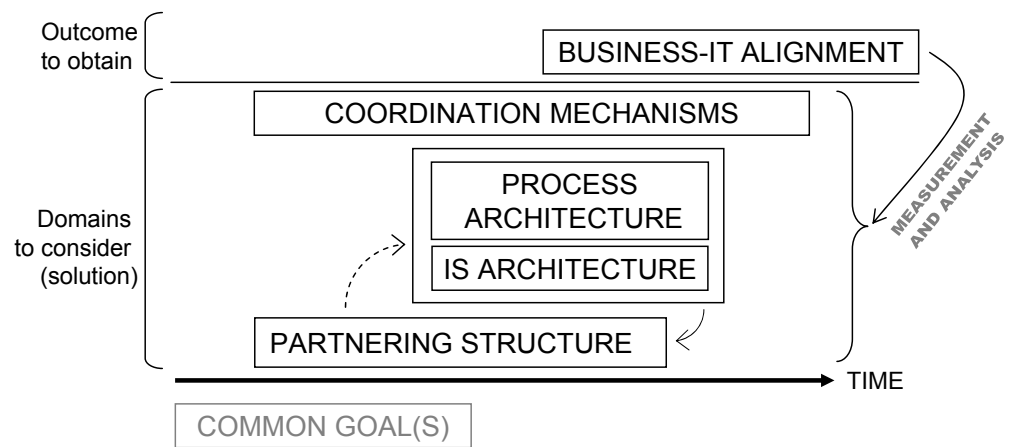

Figure 10.3: Claim derived from the presented research (adapted from Figure 6.8).

Regardless of how a CNO defines its partnering structure, the IS/process architects in the CNO must (i) consider such a structure for the design of the IS architecture and process architecture, and (ii) make sure that a good communication exists between the partnering structure team and the IS architecture and process architecture teams. This is necessary because the partnering structure design produces constraints that need to be met by the IS architecture and process architecture designs.

Hypothesis 1 Effective communication between the partnering structure team and the IS architecture and process architecture teams is necessary to create understanding and to achieve B-ITa in a CNO.

The small curved arrow starting from the IS architecture and process architecture domains and pointing to the partnering structure in Figure 10.3 asserts that, for example, it is possible to first design an IS architecture and later readjust the design of the partnering structure in a way that ensures the crucial fit between the structure and the IS architecture. Then, later it can be possible to check whether the constraints/requirements resulting from this partnering structure mandate some adjustment in the IS architecture. This situation represents a feedback loop that plays an important role in the enhancement of the domains when achieving B-ITa.

Hypothesis 2 The partnering structure domain is enhanced when a feedback loop helps to convey some insights when/after designing the adjacent IS architecture and process architecture domains.

Finally, the remaining arrow starting from the "outcome to obtain" side and pointing to the "domains to consider (solution)" side in Figure 10.3 represents a feedback process. When achieving B-ITa, measurement and analysis processes help to define measures (e.g., financial and internal) and communicate the BITa results in order to consider them in future B-ITa projects to 'assure' quality 
and real improvements. This feedback process is to improve and evolve domains through experience.

Hypothesis 3 The four B-ITa domains are enhanced when measurement and analysis processes take place and a feedback process communicates the B-ITa results of the CNO to the involved teams.

Our fourth and last hypothesis covers a set of claims that the final version of our ICoNOs MM involves (see Figure 10.2). This hypothesis has the form of

$$
X \longrightarrow Y \longrightarrow Z
$$

where $\mathrm{X}$ stands for the set of specific goals include in a B-ITa process area, $\mathrm{Y}$ stands for a particular level that a CNO wants to achieve, and Z stand for B-ITa. A specific claim using this hypothesis structure is

If a CNO strives to have an standardized IS architecture domain, then such a CNO must achieve all goals covered by all B-ITa process areas at level 3 in the particular IS architecture domain. Reaching level 3 in the IS architecture domain results in B-ITa improvements in such a CNO so that value is created for all participating organizations.

In our research we have not conducted a longitudinal case study in order to verify if the consideration of our ICoNOs MM recommendations (in the form of process areas, specific goals and practices) indeed results in B-ITa improvements.

We believe that all these hypotheses can help to lay down the basis for a research agenda to provide more understanding of the alignment topic in crossorganizational environments. In this context, a number of opportunities exists for future research. First, each hypothesis can be validated by means of case studies in order to find evidence to support or refute it. Second, one can explore in more detail the implications of the new claim derived from the study presented in this dissertation for practitioners. This can help to translate the hypotheses into actionable guidelines which could help B-ITa project stakeholders improve the chances of success. 

Appendices 



\section{Appendix A Articles upon this dissertation is based}

- Santana Tapia, R., 2009, ICoNOs MM: The IT-enabled collaborative networked organizations maturity model. In: Leveraging knowledge for innovation in Collaborative Networks, 10th IFIP Working Conference on Virtual Enterprises, 7-9 Oct 2009, Thessaloniki, Greece. Springer Verlag.

- Santana Tapia, R., 2009, Coordination and partnering structure are vital domains in collaborative business-IT alignment: Elaborating on the ICoNOs MM. In: Proceedings of the 4th IEEE/IFIP International Workshop on Business-Driven IT Management (BDIM 2009) in conjunction with The 11th IFIP/IEEE International Symposium on Integrated Network Management. 1 Jun 2009, New York, USA, pp. 73-74. IEEE Computer Society Press. ISBN 978-1-4244-3923-2

- Santana Tapia, R., 2009, Converging on business-IT alignment best practices: Lessons learned from a Dutch cross-governmental partnership. In: Proceedings of the 15th International Conference on Concurrent Enterprising (ICE 2009) "Collaborative Innovation: Emerging Technologies, Environments and Communities", 22-24 Jun 2009, Leiden, The Netherlands.

- Santana Tapia, R., Daneva, M., van Eck, P., Castro Cárdenas, N., van Oene, L., 2008, Business-IT alignment domains and principles for networked organizations: A qualitative multiple case study. In: On the Move to Meaningful Internet Systems, OTM 2008 Workshops Proceedings. 9-14 Nov 2008, Monterrey, Mexico, pp. 241-252. Springer Berlin/Heidelberg.

- Santana Tapia, R., Daneva, M., van Eck, P., Wieringa, R., 2008, Towards a business-IT alignment maturity model for collaborative networked organizations. In: Proc. of the International Workshop on Enterprise Interoperability (IWEI 2008) in conjunction with The Twelfth IEEE International EDOC Conference. 18 Sept 2008, Munich, Germany, pp. 70-81. CTIT.

- Santana Tapia, R., van Eck, P., and Daneva, M., 2008, Validating the domains of an inter-organizational business-IT alignment assessment instrument: A case study. Technical Report TR-CTIT-08-53 Centre for Telematics and Information Technology, University of Twente, Enschede. ISSN 1381-3625

- Santana Tapia, R. and van Oene, L., 2008, Some empirical evidence on business-IT alignment processes in the public sector: A case study report. Technical Report TR-CTIT-08-46 Centre for Telematics and Information Technology, University of Twente, Enschede. ISSN 1381-3625

- Santana Tapia, R. and Zarvic, N., 2008, Value-based partnering structure design for networked businesses: A multi-method approach. In: Proceedings of the 21st Bled Conference "eCollaboration" Overcoming Boundaries through MultiChannel Interaction, 15-18 Jun 2008, Bled, Slovenia, pp. 263-276. Kranj: Moderna organizacija. ISBN 978-961-232-217-5

- Santana Tapia, R., Daneva M., van Eck P., 2007, Validating adequacy and suitability of business-IT alignment criteria in an inter-enterprise maturity model. In: Proceedings of the Eleventh IEEE International EDOC Enterprise Computing Conference, 15-19 Oct 2007, Annapolis, MD, USA. pp. 202-213. IEEE Computer Society Press. ISBN 0-7695-2891-0 
- Santana Tapia, R., 2007, Developing a maturity model for IT alignment in a cross-organizational environment. In SIKS Proceedings of the 2nd Dutch/Belgian Conference on Enterprise Information Systems, Groningen, The Netherlands.

- Santana Tapia, R., Daneva M., van Eck P., 2007, Developing an inter-enterprise alignment maturity model: Research challenges and solutions. In C. Rolland, O. Pastor and J.-L. Cavarero, editors, Proceedings of the 1st International Conference on Research Challenges in Information Science (RCIS'07), 23-26 April, Ouarzazate, Morocco, pp. 51-59.

- Santana Tapia, R., 2006, A Value-Based Maturity Model for IT Alignment in Networked Businesses, In: Proceedings of Workshops and Doctoral Contsortium of the 18th International Conference on Advanced Information Systems Engineering (CAISE'06), 5-9 Jun 2006, Luxembourg, Grand Duchy of Luxembourg. pp. 1201-1208. Presses Universitaires de Namur. ISBN 2-87037-525-5

- Santana Tapia, R., 2006, What is a Networked Business?, Technical Report Num. TR-CTIT-06-23a, Centre for Telematics and Information Technology, University of Twente, The Netherlands. 


\section{Appendix B \\ Generic goals and practices in the CMMI}

\section{GG1 Achieve Specific Goals}

The process supports and enables achievement of the specific goals of the process area by transforming identifiable input work products to produce identifiable output work products.

GP1.1 Perform Specific Practices

Perform the specific practices of the process area to develop work products and provide services to achieve the specific goals of the process area.

\section{GG2 Institutionalize a Managed Process}

The process is institutionalized as a managed process.

GP2.1 Establish an Organizational Policy

Establish and maintain an organizational policy for planning and performing the process.

GP2.2 Plan the Process

Establish and maintain the plan for performing the process.

GP2.3 Provide Resources

Provide adequate resources for performing the process, developing the work products, and providing the services of the process.

GP2.4 Assign Responsibility Assign responsibility and authority for performing the process, developing the work products, and providing the services of the process.

GP2.5 Train People

Train the people performing or supporting the process as needed.

GP2.6 Manage Configurations

Place designated work products of the process under appropriate levels of control.

GP2.7 Identify and Involve Relevant Stakeholders

Identify and involve the relevant stakeholders of the process as planned.

GP2.8 Monitor and Control the Process

Monitor and control the process against the plan for performing the process and take appropriate corrective action.

GP2.9 Objectively Evaluate Adherence

Objectively evaluate adherence of the process against its process description, standards, and procedures, and address noncompliance.

GP2.10 Review Status with Higher Level Management

Review the activities, status, and results of the process with higher level management and resolve issues.

\section{GG3 Institutionalize a Defined Process}

The process is institutionalized as a defined process. 


\section{APPENDIX B: GENERIC GOALS AND PRACTICES IN THE CMMI}

GP3.1 Establish a Defined Process

Establish and maintain the description of a defined process.

GP3.2 Collect Improvement Information

Collect work products, measures, measurement results, and improvement information derived from planning and performing the process to support the future use and improvement of the organizations processes and process assets.

\section{GG4 Institutionalize a Quantitatively Managed Process}

The process is institutionalized as a quantitatively managed process.

GP4.1 Establish Quantitative Objectives for the Process

Establish and maintain quantitative objectives for the process, which address quality and process performance, based on customer needs and business objectives.

GP4.2 Stabilize Subprocess Performance

Stabilize the performance of one or more subprocesses to determine the ability of the process to achieve the established quantitative quality and process-performance objectives.

\section{GG5 Institutionalize an Optimizing Process}

The process is institutionalized as an optimizing process.

GP5.1 Ensure Continuous Process Improvement

Ensure continuous improvement of the process in fulfilling the relevant business objectives of the organization.

GP5.2 Correct Root Causes of Problems

Identify and correct the root causes of defects and other problems in the process. 


\section{Appendix C \\ Questionnaire used in the case study 5}

1. Positie van uw organisatie in de omgevingsvergunning netwerkorganisatie.

In de centrale doelstelling van deze vragen is te zien hoe de deelnemende organisaties zijn eigen positie binnen de netwerkorganisatie bepaalt.

A Wat zijn de strategische doelstellingen om deel van de omgevingsvergunning netwerkorganisatie uit te maken (d.w.z., uitbreiding van marktbereik, ontwikkeling van de gevorderde diensten, zich vertrouwd maken met een nieuwe technologie, deelneming aan een experiment, etc.)?

B Wat zijn de competenties, specifieke activa, en middelen van uw organisatie die voor het werken binnen de omgevingsvergunning netwerkorganisatie essentieel zijn?

$\mathrm{C}$ Hoe belangrijk is de omgevingsvergunning voor uw organisatie

2. Perceptie van de omgevingsvergunning netwerkorganisatie door uw organisatie.

Deze vragen en onderwerpen bespreken de perceptie van de omgevingsvergunning netwerkorganisatie vanuit het perspectief van de genterviewde.

\begin{tabular}{|c|c|c|}
\hline \multirow{10}{*}{ 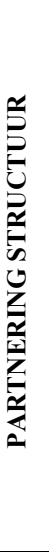 } & \multicolumn{2}{|r|}{ Kenmerken van de omgevingsvergunning netwerkorganisatie. } \\
\hline & 1 & Worden de gemeenschappelijke doelstelling(en) gespecificeerd? \\
\hline & 2 & $\begin{array}{l}\text { Kent iedereen de omgevingsvergunning netwerkorganisatie (actoren, kleine of } \\
\text { grote spelers, bevoegd gezag, etc.).? }\end{array}$ \\
\hline & 3 & $\begin{array}{l}\text { Is er een ontwerp van de omgevingsvergunning netwerkorganisatie om die als } \\
\text { volledig systeem (d.w.z., actoren, relaties, input, output, etc.) te beschrijven? }\end{array}$ \\
\hline & \multicolumn{2}{|r|}{ Rollen en verantwoordelijkheden. } \\
\hline & 4 & $\begin{array}{l}\text { Zijn er beschreven service level agreements over de deliverables, de kwaliteit en } \\
\text { het werk van elke deelnemende organisatie? }\end{array}$ \\
\hline & 5 & $\begin{array}{l}\text { Was de definitie van een samenwerking structuur noodzakelijk om efficiënt sa- } \\
\text { men te kunnen werken? }\end{array}$ \\
\hline & 6 & Welke rollen en actoren zijn noodzakelijk? Wie neemt zorg voor wat? \\
\hline & 7 & $\begin{array}{l}\text { Hoe worden de organisatorische middelen (mensen, materialen, en informatie) } \\
\text { verdeeld binnen de omgevingsvergunning netwerkorganisatie? }\end{array}$ \\
\hline & 8 & $\begin{array}{l}\text { Is er aandeelrisico- en beloningen beleid dat wordt gevestigd om wederzijdse } \\
\text { voordelen en verplichting te verhogen? }\end{array}$ \\
\hline
\end{tabular}

\begin{tabular}{|c|c|c|}
\hline \multicolumn{3}{|c|}{ Interfaces tussen organisaties. } \\
\hline \multirow{6}{*}{ 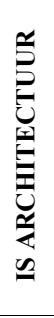 } & 1 & $\begin{array}{l}\text { Is er een beschrijving van de huidige situatie van de systemen? Zo ja, hoe is die } \\
\text { ontwikkeld? }\end{array}$ \\
\hline & 2 & Wat zijn de noodzakelijke technologienormen en principes in het IS gebied? \\
\hline & 3 & $\begin{array}{l}\text { Hoe wordt bepaald of de systemen alle essentiële informatieverwerkingsbehoef- } \\
\text { ten steunen? (gap analyse) }\end{array}$ \\
\hline & 4 & Hoe worden de interfaces tussen IS bij voorkeur gerealiseerd? \\
\hline & 5 & $\begin{array}{l}\text { Steunen de bestaande IS het realiseren van interfaces? Zo nee, hoe wordt het dan } \\
\text { opgelost? }\end{array}$ \\
\hline & 6 & Vindt er een beheer van IS en interfaces plaats? Zo nee, zal dat gebeuren? en hoe? \\
\hline
\end{tabular}


APPENDIX C: QUESTIONNAIRE USED IN THE CASE STUDY 5

\begin{tabular}{|c|c|c|}
\hline \multicolumn{3}{|c|}{ Interfaces tussen organisaties. } \\
\hline \multirow{6}{*}{ 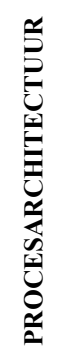 } & 1 & Is er de huidige situatie van de processen beschreven? Zo ja, hoe is die ontwikkeld? \\
\hline & 2 & $\begin{array}{l}\text { Wat zijn de informatiestromen, en processen, die nodig zijn om de omgevings- } \\
\text { vergunning te leveren? Kent iedereen met een rol in het Wabo proces die informa- } \\
\text { tiestromen en processen? Zo nee, hoe gaat dit georganiseerd worden? }\end{array}$ \\
\hline & 3 & Hoe worden de processen tussen de organisaties in kaart gebracht? \\
\hline & 4 & $\begin{array}{l}\text { Is er een evaluatie/ selectie/ ontwerp of processen die nodig zijn om de gewenste } \\
\text { situatie te steunen? }\end{array}$ \\
\hline & 5 & $\begin{array}{l}\text { Hoe gaan jullie als samenwerkende organisaties ervoor zorgen dat de procesbe- } \\
\text { schrijvingen actueel blijven? }\end{array}$ \\
\hline & 6 & Vindt er een beheer van procesportfolio plaats? Zo nee, zal dat gebeuren? en hoe? \\
\hline
\end{tabular}

\begin{tabular}{|c|c|c|}
\hline \multirow{6}{*}{ 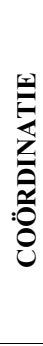 } & \multicolumn{2}{|r|}{ Kenmerken van de omgevingsvergunning netwerkorganisatie. } \\
\hline & 1 & $\begin{array}{l}\text { Wat is de graad van standaardisatie van output en handhaving tussen de deelne- } \\
\text { mende organisaties? }\end{array}$ \\
\hline & 2 & $\begin{array}{l}\text { Wordt het werk gecontroleerd door specifieke personen die de verantwoordelijk- } \\
\text { heid voor de processen nemen, instructies geven, en activiteiten controleren? }\end{array}$ \\
\hline & \multicolumn{2}{|r|}{ Interfaces tussen organisaties. } \\
\hline & 3 & $\begin{array}{l}\text { Hoe zijn de communicatie processen (d.w.z., persoonlijke, samenwerking in } \\
\text { werkgroepen, workflow-systemen, communities)? }\end{array}$ \\
\hline & 4 & $\begin{array}{l}\text { Zijn de formele/informele communicatiekanalen belangrijk om efficiënt samen te } \\
\text { kunnen werken? }\end{array}$ \\
\hline
\end{tabular}




\section{Appendix D \\ Pilot assessment questions}

\section{Partnering structure}

BDM

01 Wordt een algemene identiteit van de 3TU.Federatie beschreven? (missie, visie, middelen, product/dienst, competitive advantage)

02 Worden de gemeenschappelijke doelstelling(en) gespecificeerd?

03 Kent iedereen de externe omgeving van de 3TU.Federatie? (target market, steunende organisaties, concurrenten)

04 Is er een ontwerp van de 3TU.Federatie om die als een volledig systeem (d.w.z., actoren, relaties, input, output, etc.) te beschrijven?

05 Wordt een revenue model, koststructuur, investeringsmodel gedefinieerd?

06 Is er een algemeen plan voor de 3TU.Federatie?

\section{SLA}

07 Worden service level agreements bepaald tussen de drie universiteiten? Zo ja, tot hoever word dat gedaan? (performance thresholds, escalation procedures, penalties and incentives, etc.)

GSC

08 Is er een algemeen organigram van de 3TU.Federatie?

09 Worden besluitvormingsprocedures gespecificeerd?

10 Was de definitie van een samenwerking structuur noodzakelijk om efficiënt samen te kunnen werken?

11 Welke rollen en actoren zijn noodzakelijk? Wie neemt zorg voor wat?

12 Hoe worden de organisatorische middelen (mensen, materialen, en informatie) verdeeld binnen de 3TU.Federatie?

13 Is er aandeelrisico- en beloningen beleid dat wordt gevestigd om wederzijdse voordelen en verplichting te verhogen?

14 Zijn er specifieke programma's gedefinieerd in overeenkomst met de prioriteiten en het plan?

15 Hoe verzekert de 3 TU.Federatie de compliance van processen met contracten en doelstellingen?

\section{IS architecture}

CSA

01 Is er een beschrijving van de huidige situatie van de informatiesystemen in de 3TU.Federatie? Zo ja, hoe is die ontwikkeld?

02 Wat zijn de noodzakelijke technologienormen en principes in het IS gebied? 
IsPM

03 Word een target IS architectuur gedefinieerd? Zo ja, hoe is dat gedaan?

04 Vind IS portfolio management plaats in de 3TU.Federatie?

05 Steunen de bestaande IS het realiseren van interfaces tussen de universiteiten? Zo nee, hoe wordt het dan opgelost?

06 Vindt er een beheer van ISs plaats? Zo nee, zal dat gebeuren? en hoe?

IsCD

07 Worden de IS vaardigheden van de deelnemers geanalyseerd?

IsRM

08 Vind IS requirements management plaats in de 3TU.Federatie?

09 Hoe wordt bepaald of de systemen alle essentile informatieverwerkingsbehoeften steunen? (gap analyse)

\section{Process architecture}

CPD

01 Is er de huidige situatie van de processen beschreven? Zo ja, hoe is die ontwikkeld?

TPA

02 Wat zijn de informatiestromen, en processen, die nodig zijn om samen te kunnen werken, en om diensten te leveren in de 3TU.Federatie?

03 Kent iedereen die informatiestromen en processen? Zo nee, hoe gaat dit georganiseerd worden?

04 Hoe worden de processen tussen de universiteiten in kaart gebracht?

PFP

05 Is er een evaluatie/selectie/ontwerp of processen die nodig zijn om de gewenste situatie binnen de 3TU.Federatie te steunen?

06 Hoe gaan jullie als samenwerkende universiteiten ervoor zorgen dat de procesbeschrijvingen actueel blijven?

\section{Coordination}

DTS

01 Wordt het werk gecontroleerd door specifieke personen die de verantwoordelijkheid voor de processen nemen, instructies geven, en activiteiten controleren?

$\ln C A$

02 Hoe zijn de communicatie processen (d.w.z., persoonlijke, samenwerking in werkgroepen, workflowsystemen, communities)? 
03 Zijn de informele communicatiekanalen belangrijk om efficiënt samen te kunnen werken als 3TU.Federatie?

STD

04 Wat is de graad van standaardisatie tussen de drie universiteiten?

$\mathrm{COC}$

05 Zijn er overeenkomsten m.b.t. de communicatiekanalen en het delen van kennis binnen de 3TU.Federatie?

06 Zijn de formele communicatiekanalen belangrijk om efficiënt samen te kunnen werken? 



\section{Appendix E Answers to the post-task survey}

1. Do you consider these recommendations convincing and helpful? Why or why not?

1st Interviewee. Sure the recommendations are convincing and helpful as a list of issues which should be given attention to. But they are also so general (high level) or mayby open doors that I am wondering if the model you used is selective enough to determine the reel gaps or risks that we can encounter or have to tackle in the choosen 3TU.Federatie model.

I am also wondering if your model fits to the 3 TU.Federatie model we used. I think it will better fit to the model insite of each university. In the 3TU.Federatie level I think we excluded a lot of risks by not creating one business model, a governance structure, same processes architecture, same information systems architecture but only a set of the same user products (e.g., request for a course, or subscribe for an exam) which fits to the reality for $100 \%$ and will be delivered to all parties (i.e. user interfaces or enterprise busses) by means of a standardized communication en data exchange protocol and according to rules layed down in a service level agreement.

This reduces the need of a lot of your parameters on which your model has been built for operating and maintaining our 3TU.Federatie system. Only the set of products and standards has to be maintained (in our case by a commission).

2nd Interviewee. Mijn indruk is dat de aanbevelingen ons helpen om in het 3TU.Federatie.ICT deel op aspecten het programma aan te scherpen. De aanbevelingen zijn best 'technisch' geformuleerd. Een vertaling voor het management biedt voordelen.

3rd Interviewee. Ik mis de waarom vraag van de aanbeveling. Om die reden is het minder overtuigend (voor mij persoonlijk). Samenvattend mis ik een analyse van de gegevens die je gevonden hebt en de oplossing die je presenteert. Hierdoor komt je opolossing/aanbeveling uit de lucht vallen en maakt hem daarom niet geloofwaardig.

4th Interviewee. At reading time, your recommendations seem very useful considering that your model is the first model that can give this kind of recommendations to improve alignment issues in network organizations. However, I would like to apply the recommendations before giving a more precise answer to your question. I am convinced that some of your points can be discussed in our meetings.

5th Interviewee. Yes, they give a direction that we must go to achieve a better business-IT alignment.

6th Interviewee. De aanbevelingen zijn redelijk algemeen, maar op 1 en 8 na goed voor het nadenken over waar je naartoe zou willen en hoe je daar zou moeten komen. 1 is een lastige voor een organisatie als een universiteit. Volgens mij komt dat omdat de overheid/regelgeving/overheidsbeleid een belangrijke rol speelt (ben wel eens een model om hiermee om te gaan 
tegengekomen in "IT governance : how top performers manage IT decision rights for superior results / Peter Weill and Jeanne W. Ross"). Dus met 1 kan ik weinig. 8 gebeurt al voor zover mogelijk op dit moment, maar in lijn met de diepgang van de samenwerking.

2. Do you think that you could achieve business-IT alignment without being aware of the results (presented in the Table 9.1) and specifically without recommendations $1,2,3$ and 8$)$ ? Why or why not?

Consider the following definition of alignment when answering this question: Business-IT alignment is the process to make the services offered by information system support the requirements of the networked organization derived from analyzing its goal(s) and processes, and the relations between the participating organizations.

1st Interviewee. Yes, the goals of business-IT alignment are first: is it useful, does it what I want and how I want it, always and everywhere. Secondly is it at minimum costs. Of course recommendations like 1, 2, 3 and 8 contribute to a perfect and efficient operation and maintenance. In addition, for the 3TU.Federatie the business-IT alignment definition should stop after the word goals. Processes and relations are not important for 3TU.

2nd Interviewee. Ik zou wel eens een voorbeeld willen zien van een 'multi-value configuration', zoals genoemd in aanbeveling 1 . Ik heb geen goed beeld van wat we daarmee zouden kunnen, temeer omdat nu in 3TU-verband niet zozeer economische motieven een rol spelen, maar kwalitatieve mogelijkheden voor studenten in beeld zijn. Aanbevelingen 2 en 3 stimuleren ons tot aanscherping van het overleg- en aansturingsmodel. Aanbeveling 8 vind ik een erg goede.

3rd Interviewee. Ja hoor, ook zonder dit model kan dat. Dat zou wel een hele groet eer zijn voor dit model.

4th Interviewee. It could be possible but with your recommendations we could do it properly. Personally, I think recommendation 3 is very important. As you know, the implementation of a governance model for the Architecture Workgroup has been left aside.

5th Interviewee. I don't think that it is possible without this recommendations to make the 3TU.Federatie collaboration better in control and to realize the efficiency and results that we expect.

6th Interviewee. De resultaten in de tabel helpen focusseren op relevante issues. Wat ik merk is dat je tijdens de pogingen om op operationeel vlak een gezamenlijke dlwo van de grond te krijgen, deze punten ook grotendeels vanzelf naar de oppervlakte komen.

3. Are recommendations 4, 5, 6 and 7 useful for the 3TU Federatie in its actual operational stage? Why or why not?

1st Interviewee. No but they are important for the universities itself.

2nd Interviewee. Aan het verbeteren van de 'IS capabilities' (4) zijn we aan het werken. Zo ook (6). Het verschil met TU/e is dat TUD en UT het applicatielandschap nog aan het wijzigen zijn (Osiris, Blackboard, Syllabus, 
...). Aanbeveling 5 vind ik weer zo'n 'technische'. Ik kan me er nog geen goede voorstelling van maken. Aan (7) werken we door nu de 'roadmap' tot 1-1-2011 te expliciteren.

3rd Interviewee. Nee, dit past niet bij de 3TU gedachte. Wel per universiteit

4th Interviewee. Yes, but at university level. I think each university must follow these recommendations. For the 3TU, they are less important. But I believe that this is not the case of other network organizations (which certainly could get benefits from these recommendations).

$\mathbf{5 t h}$ Interviewee. I think that this recommendations are very important to get more understanding between the business and IT. They offer also the possibility to get a better collaboration between the 3TU business-organisations and the IT-organizations.

6th Interviewee. $4 \mathrm{t} / \mathrm{m} 7$ zijn het meest concreet en voor mij meest herkenbaar, dus mijn antwoord is volmondig ja. Aanbeveling 7 is nog ver weg, dus voor 7 geldt het wat minder. De reden is dat het al lastig genoeg is om bestaande processen op elkaar aan te sluiten, dus aan procesoptimalisatie zijn we nog niet toe.

4. The ICoNOs MM has (still) no tool for conducting assessments. Therefore, we used interviews and documents reviews that helped us to present qualitative results. Did you find this assessment manner appropriated? Or would you prefer a tool for obtaining quantitative results providing numerical descriptions rather than narrative recommendations?

1st Interviewee. As I stated already at the first question its too general, so I prefer more quantitative results.

2nd Interviewee. Ik heb op zich geen probleem met de gehanteerde werkwijze. Zoals eerder gezegd, ik vind de aanbevelingen soms te 'technisch' geformuleerd. Als ze bedoeld zijn voor het management dan verdient de wijze van formuleren en uitwerken aandacht.

3rd Interviewee. Ik vindt een kwaantitatieve aanpak niet altijd noodzakelijk, mits er een juiste analyse is.

4th Interviewee. Personally, I prefer qualitative results. Quantitative results can be a little bit difficult to understand to implement them. So, yes, I find this assessment manner appropriated. I have only a tip: you know you did not ask so many questions. However, you thought that you had answers to your most important questions. Maybe asking all your questions in a strict order could help you to get more data and to order properly the answers of all of us.

5th Interviewee. I find the used method with interviews a little bit weak. Reason therefore is that I had no overall picture how to place the questions in relation with the mm survey. Therfore I like a tool more.

6th Interviewee. In ons stadium vind ik documenten en interviews het nuttigst. Voor kwantiatieve analyse is volgens mij meer kennis van het begrippenkader en meer ervaring in het samenwerkingsproces nodig. 
5. Would you use and recommend to others the use of the ICoNOs MM for this kind of assessment?

1st Interviewee. Not at this time, I rather should pilot the model first within a university.

2nd Interviewee. Ik denk dat het instrument nog 'management-proof' gemaakt moet worden.

3rd Interviewee. Misschien. De basis is ok. Maar de adviezen heb ik eerst 3x door moeten lezen voordat ik ze begreep. Kortom: het abstracte taalgebruik in combinatie met de compactheid maakt het moeilijk leesbaar. Ik herken wel je invulling, maar kan zelfg niet beoordelen of de vinkjes wel allemaal goed staan. Ik weet immers niet waar al die afkortingen in dit model voor staan.

Je conclusies en adviezen komen hierdoor voor mij uit de lucht vallen. Het lijkt alsof je adviezen geeft, voor zaken waar ikzelf (nog) geen probleem zie. Echter: als ik heel goed je adviezen lees, dan kan ik tussen de regels door wel lezen welke problemen je probeert te adresseren.

4th Interviewee. Before recommend its use, I would like to us it and to know much more about it. However, (based on the importance of some of the recommendations you gave us) I would probably recommend it.

5th Interviewee. This kind of making assements is very important to get insights in the maturity of network organizations. So my answer is 'yes' this model gives us more insights in how things are running en wat we can do to make ist better.

6th Interviewee. Ik denk dat dit een nuttig instrument kan zijn, maar heb ook geen vergelijkingsmateriaal met andere methoden voor dit soort analyses. Het aangeven van een maturity level gekoppeld aan een aantal inhoudelijke parameters vind ik erg nuttig en inzichtelijk. 


\section{Bibliography}

[1] S. Abrahao and G. Poels. Experimental evaluation of an object-oriented function point measurement procedure. Information and Software Technology, 49(1):366$380,2007$.

[2] O. Adelakun. IT outsourcing maturity model. In Proceedings of the 12th European Conference on Information Systems, The European IS Profession in the Global Networking Environment (ECIS'04), pages 13-21, June 2004.

[3] R. Allen. Workflow: An introduction. In L. Fischer, editor, The Workflow Handbook 2001, pages 15-38. Lighthouse Point, Fla.: Future Strategies, Book Division, 2001.

[4] L. M. Applegate, F. W. McFarlan, and J. L. McKenney. Corporate Information Systems Management: Text and Cases. Irwin Publishings, fourth edition, 1996.

[5] A. April, J. H. Hayes, A. Abran, and R. Dumke. Software maintenance maturity model $\left(\mathrm{SM}^{m m}\right)$ : the software maintenance process model. Journal of Software Maintenance and Evolution: Research and Practice, 17(3):197-224, 2005.

[6] S. Aral and P. Weill. IT assets, organizational capabilities, and firm performance: How resource allocations and organizational differences explain performance variation. Organization Science, 18(5):763-780, 2007.

[7] J. D. Aram. Concepts of interdisciplinarity: Configurations of knowledge and action. Human Relations, 57(4):379-412, 2004.

[8] W. F. Averyt and K. Ramagopal. Strategic disruption and transaction cost economics - the case of the american auto industry and japanese competition. International Business Review, 8(1):39-53, 1999.

[9] W. R. J. Baets. Aligning information systems with business strategy. Journal of Strategic Information Systems, 1(4):205-213, 1992.

[10] W. R. J. Baets. Some empirical evidence on IS strategy alignment in banking. Information and Management, 30(4):155-177, 1996.

[11] L. M. Baker. Observation: a complex research method. Library Trends, 55(1):171189, 2006.

[12] F. M. Barbini and A. D'Atri. How innovative are virtual enterprises? In D. B. et al., editor, Proceedings of the 13th European Conference on Information Systems, Information Systems in a Rapidly Changing Economy (ECIS'05), May 2005 .

[13] S. Beecham, T. Hall, C. Britton, M. Cottee, and A. Rainer. Using an expert panel to validate a requirements process improvement model. Journal of Systems and Software, 76(3):251-275, 2005.

[14] I. Beeson, S. Green, J. Sa, and A. Sully. Linking business processes and information systems provision in a dynamic environment. Information Systems Frontiers, 4(3):317-329, 2002.

[15] I. Benbasat, D. Goldstein, and M. Mead. The case study research strategy in studies in information systems. MIS Quarterly, 11(3):369-388, 1987.

[16] H. Benbya and B. McKelvey. Using coevolutionary and complexity theories to improve IS alignment: A multi-level approach. Journal of Information Technology, 21(4):284-298, 2006.

[17] R. Benyon and R. Johnson. Service Agreements: A Management Guide. Van Haren Publishing, 2006.

[18] A.-J. Berre, B. Elvester, N. Figay, C. Guglielmina, S. G. Johnsen, D. Karlsen, T. Knothe, and S. Lippe. The ATHENA Interoperability Framework. In R. J. Gonalves, J. P. Müller, K. Mertins, and M. Zelm, editors, Enterprise Interoperability II, pages 569-580. Springer London, 2007. 
[19] K. S. Birdi, M. G. Patterson, and S. J. Wood. Learning to perform? A comparison of learning practices and organizational performance in profit- and non-profitmaking sectors in the UK. International Journal of Training and Development, 11(4):265-281, 2007.

[20] S. J. Bleistein, K. Cox, and J. Verner. Validating strategic alignment of organizational IT requirements using goal modeling and problem diagrams. Journal of Systems and Software, 79(3):362-378, 2006.

[21] Blue-Crow. Business process modelling and analysis, 2007.

[22] L. Bodenstaff, A. Wombacher, and M. U. Reichert. On formal consistency between value and coordination models. Technical Report TR-CTIT-07-91, University of Twente, Enschede, October 2007.

[23] B. W. Boehm and K. J. Sullivan. Software economics: A roadmap. In in The Future of Software Engineering, Proceedings of the 22nd International Conference on Software Engineering, pages 319-343. ACM Press, 2000.

[24] J. R. J. Boland. Phenomenology: A preferred approach to research on information systems. In Trends in information systems, pages 341-349. North-Holland Publishing Co., Amsterdam, The Netherlands, 1986.

[25] J. Bouman, J. Trienekens, and M. van der Zwan. Specification of service level agreements, clarifying concepts on the basis of practical research. In STEP '99: Proceedings of the Software Technology and Engineering Practice, pages 169-178, Washington, DC, USA, 1999. IEEE Computer Society.

[26] L. Boyd. Explanation versus intervention in complex systems, 2003. White Paper.

[27] G. A. Boyne. Public and private management: Whats the difference? Journal of Management Studies, 39(1):97-122, 2002.

[28] P. Brereton, B. A. Kitchenham, D. Budgen, M. Turner, and M. Khalil. Lessons from applying the systematic literature review process within the software engineering domain. Journal of Systems and Software, 80(4):571-583, 2007.

[29] M. Broadbent and E. Kitzis. Interweaving business-driven IT strategy and execution: Four foundation factors. Ivey Business Journal, 69(3):1-6, 2005.

[30] M. Broadbent and P. Weill. Improving business and information strategy alignment: Learning from the banking industry. IBM Systems Journal, 32(1):162-179, 1993.

[31] K. Bruce. Can you align IT with business strategy? Strategy $\&$ Leadership, 26(5):16-21, 1998.

[32] A. Bryman. Integrating quantitative and qualitative research: how is it done? Qualitative Research, 6(1):97-113, 2006.

[33] R. Buday. SABRE gives the edge to American Airlines. Information Week, pages 35-35, May 1986.

[34] D. Budgen and P. Brereton. Performing systematic literature reviews in software engineering. In ICSE '06: Proceedings of the 28th international conference on Software engineering, pages 1051-1052, New York, NY, USA, 2006. ACM Press.

[35] M. Buelens and H. van den Broeck. An analysis of differences in work motivation between public and private sector organizations. Public Administration Review, 67(1):65-74, 2007.

[36] B. Burke. Evolving architecture maturity, 2002.

[37] J. M. Burn and C. Szeto. A comparison of the views of business and IT management on success factors for strategic alignment. Information $\&$ Management, $37(4): 197-216,2000$. 


\section{BIBLIOGRAPHY}

[38] L. M. Camarinha-Matos and H. Afsarmanesh. Collaborative Networked Organizations: A Research Agenda for Emerging Business Models. Kluwer Academic Publishers, 2004.

[39] L. M. Camarinha-Matos and H. Afsarmanesh. The emerging discipline of collaborative networks. In Virtual Enterprises and Collaborative Networks, pages 3-16. Kluwer Academic Publishers, 2004.

[40] B. Campbell. Alignment: Resolving ambiguity within bounded choices. In Proceedings of the PACIS 2005, pages 1-14, Bangkok, Thailand, 2005.

[41] B. Campbell, R. Kay, and D. Avison. Strategic alignment: A practitioners perspective. Journal of Enterprise Information Management, 18(5/6):653-664, 2005.

[42] Canadian Council for Refugees. Best settlement practices, 1998. Retrieved October 15, 2008 from http://ccrweb.ca/bpfina1.pdf.

[43] Canadian Council for Refugees. Canadian national settlement service standards framework, 2000. Retrieved Oct 15, 2008 from http://ccrweb.ca/standards.pdf.

[44] N. G. Carr. IT doesn't matter. Harvard Business Review, pages 41-49, May 2003.

[45] J. I. Cash Jr. and B. R. Konsynski. IS redraws competitive boundaries. Harvard Business Review, pages 134-142, 1985. March-April.

[46] C. Castelfranchi. Modelling social action for AI agents. Artificial intelligence, 103, 1998.

[47] J. Champy. X-engineering the corporation: Reinventing your business in the digital age. Warner Books, 2002.

[48] Y. E. Chan. Why haven't we mastered alignment? the importance of the informal organization structure. MIS Quarterly Executive, 1(21):76-112, 2002.

[49] Y. E. Chan and S. L. Huff. Investigating information systems strategic alignments. In Proceedings of the 14th International Conference on Information Systems, pages 345-363, Vancouver, Canada, 1993.

[50] Y. E. Chan, S. L. Huff, D. W. Barclay, and D. G. Copeland. Business strategic orientation, information systems strategic orientation, and strategic alignment. Information Systems Research, 8(2):125-150, 1997.

[51] Y. E. Chan and B. H. Reich. IT alignment: An annotated bibliography. Journal of Information Technology, 22(4):316-396, 2007.

[52] Y. E. Chan and B. H. Reich. IT alignment: What have we learned? Journal of Information Technology, 22(4):297-315, 2007.

[53] Y. Chen, S. Iyer, X. Liu, D. Milojicic, and A. Sahai. SLA decomposition: Translating service level objectives to system level thresholds. In ICAC'0\%: Proceedings of the Fourth International Conference on Autonomic Computing, page 3, Washington, DC, USA, 2007. IEEE Computer Society.

[54] M. B. Chrissis, M. Konrad, and S. Shrum. CMMI: Guidelines for process integration and product improvement. Addison-Wesley, 2003.

[55] Citizenship and Immigration Canada. Immigration-contribution accountability measurement system: Security requirements for service provider organizations, 2002. Retrieved October 15, 2008 from http://integration-net.ca/english/ini/cafcipc/doc/r300-index.htm.

[56] R. Clarke and T. McGuinness. The Economics of the Firm. Basil Blackwell, repr. edition, 1993.

[57] CMMI Product Team. CMMI for Development, Version 1.2: Improving processes for better products, 2006.

[58] CMMI Team at Carnegie Mellon University. Capability Maturity Model Integration (CMMI) Version 1.2 Overview, 2007. 
[59] F. Coallier and R. Gérin-Lajoie. Open government architecture: The evolution of De Jure standards, consortium standards and open source software (CIRANO Project Report Num. 2006rp-02), 2006.

[60] R. H. Coase. The nature of the firm. In The Firm, the market, and the law, pages 33-55. University of Chicago Press, 1988. First published in Economica (4), 1937, pp.386-405.

[61] L. Cohen, L. Manion, and K. Morrison. Research Methods in Education. Routledge Falmer, sixth edition, 2007.

[62] J. Collins, N. Macehiter, D. Vile, and N. Ward-Dutton. The technology garden: Cultivating sustainable IT-business alignment. John Wiley \& Sons Ltd., 2007.

[63] Commons Group. OCASI, settlement sector database needs study: Final report, 2004.

[64] C. Conrad and M. S. Poole. Strategic Organizational Communication in a Global Economy. Harcourt College Publishers, fifth edition, 2002.

[65] K. S. Cook. Exchange and power in networks of interorganizational relations. The Sociological Quarterly, 18(1):62-82, 1977.

[66] D. R. Cooper and P. S. Schindler. Business Research Methods. Boston, [Mass., etc.]: McGraw-Hill, 8th edition, 2003.

[67] D. Court. Reflection and validity in qualitative research. Academic Exchange Quarterly, 10(1):211-214, 2006.

[68] J. W. Creswell and D. L. Miller. Determining validity in qualitative inquiry. Theory into Practice, 39(3):124-130, 2000.

[69] I. Crinson. A realist approach to the analysis of focus group data, 2001. St. Georges Hospital Medical School. University of London. Paper presented at the 5th Annual IACR Conference, held at Roskilde University, Denmark.

[70] A.-M. Croteau and F. Bergeron. An information technology trilogy: business strategy, technological deployment and organizational performance. Journal of Strategic Information Systems, 10(2):77-99, 2001.

[71] D. Damian. Stakeholders in global requirements engineering: Lessons learned from practice. IEEE Software, 24(2):21-27, 2007.

[72] M. Daneva and R. Wieringa. Requirements engineering for cross-organizational ERP implementation: Undocumented assumptions and potential mismatches. In RE'05: Proceedings of the 13th IEEE Int. Conference on Requirements Engineering, pages 63-74, Los Alamitos, CA, USA, 2005. IEEE Computer Society.

[73] M. Daneva and R. Wieringa. A coordination complexity model to support requirements engineering for cross-organizational ERP. In RE'06: Proceedings of the 14th IEEE International Requirements Engineering Conference, Minneapolis, MN, USA, 2006.

[74] D. Davies and J. Dodd. Qualitative research and the question of rigor. Qualitative Health Research,, 12(2):279-289, 2002.

[75] A. M. Davis and A. M. Hickey. Requirements researchers: Do we practice what we preach? Requirements Engineering, 7(2):107-111, 2002.

[76] E. W. Davis and R. E. Spekman. The Extended Enterprise: Gaining Competitive Advantage through Collaborative Supply Chains. Financial Times Prentice Hall, 2003.

[77] F. D. Davis. Technology Acceptance Model for Empirically testing new end-user information systems theory and results. PhD thesis, Massachusetts Institute of Technology, Cambridge, MA, USA, 1986.

[78] P. Davis and P. Wright. A realist evaluation approach to understanding the best value review process. Local Government Studies, 30(3):423-440, 2004. 


\section{BIBLIOGRAPHY}

[79] A. C. B. de Aquino and M. S. Pagliarussi. A heuristic method for composing a literature review. In Proceedings of the 30th Annual Congress of the European Accounting Association. Lisboa: European Accounting Association, 2007.

[80] D. de Koning and P. van der Marck. IT Zonder Hoofdpijn: Een Leidraad voor het Verbeteren van de Bedrijfsprestaties. Prentice Hall, 2002. In Dutch.

[81] K. Decker and V. Lesser. Analyzing a quantitative coordination relationship. Group Decision and Negotiation, 2(3):195-217, 1993.

[82] P. Decoito and L. Williams. Setting the course: A framework for coordinating services for immigrants and refugees in the peel region, 2000.

[83] D. C. Dennett. Cognitive science as reverse engineering: Several meanings of "top-down" and "bottom-up". In D. Prawitz, B. Skyrms, and D. Westerstahl, editors, Proceedings of the 9th International Congress of Logic, Methodology and Philosophy of Science, Uppsala, Sweden, 1991.

[84] D. Denyer, D. Tranfield, and J. E. van Aken. Developing design propositions through research synthesis. Organization Studies, 29(3):393-413, 2008.

[85] Z. Derzsi and J. Gordijn. A framework for business/IT alignment in networked value constellations. In T. Latour and M. Petit, editors, Proceedings of the workshops of the 18th International Conference on Advanced Information Systems Engineering (CAiSE 2006), pages 219-226, Namur, B, 2006. Namur University Press.

[86] M. Diaz and J. Sligo. How software process improvement helped Motorola. IEEE Software, 14(5):75-81, 1997.

[87] Dienst ICT. De TU/e digitale Leer- en Werkomgeving (DLWO), 2009. In Dutch.

[88] V. Dignum, F. Dignum, and L. Sonenberg. Towards dynamic reorganization of agent societies. In Proceedings of Workshop on Coordination in Emergent Agent Societies, pages 22-27, 2004.

[89] Y. Dittrich, M. John, J. Singer, and B. Tessem. Editorial for the special issue 'Qualitative Software Engineering Research'. Information and Software Technology, 49(6):531-539, 2007.

[90] DOC Enterprise IT Architecture Advisory Group. Information technology architecture: What is it, why should you care, and how do you do one?, 2004.

[91] L. M. Dooley. Case study research and theory building. Advances in Developing Human Resources, 4(3):335-354, 2002.

[92] J. Dorn, C. Grun, H. Werthner, and M. Zapletal. A survey of B2B methodologies and technologies: From business models towards deployment artifacts. In HICSS '07: Proceedings of the 40th Annual Hawaii International Conference on System Sciences, page 143, Washington, DC, USA, 2007. IEEE Computer Society.

[93] G. Dosi, R. R. Nelson, and S. G. Winter. The Nature of dynamics of organizational capabilities. Oxford University Press, 2001.

[94] R. Dubin. Theory Building. Free Press, 1978.

[95] J. Duffy. Maturity models: Blueprints for e-volution. Strategic and Leadership, 29(6):19-26, 2001.

[96] M. J. Earl, J. L. Sampler, and J. E. Short. Strategies for business process reengineering: Evidence from field studies. Journal of Management Information Systems, 12(1):31-56, 1995.

[97] B. A. Edwards. Chief executive officer behaviour: The catalyst for strategic alignment. Journal of Value-Based Management, 13(1):47-54, 2000.

[98] K. M. Eisenhardt. Building theories from case study research. The Academy of Management Review, 14(4):532-550, 1989. 
[99] K. M. Eisenhardt and M. E. Graebner. Theory building from cases: Opportunities and challenges. Academy of Management Journal, 50(1):25-32, 2007.

[100] R. M. Emerson. Power-dependence relations. American Sociological Review, 27(1):31-41, 1962.

[101] D. Farr. Service mapping, service Ontario, In: Provincial Government Conference, Lake Carling, Ontario, Canada, 2005.

[102] I. J. Farrell. Aligning IT to corporate objectives: Organisational factors in use. $\mathrm{PhD}$ thesis, Macquarie University, Sydney, 2003.

[103] Federal Architecture Working Group. Architecture Alignment and Assessment Guide. The Federal Chief Information Officer Council, 2000.

[104] D. F. Feeny and L. P. Willcocks. Core IS capabilities for exploiting information technology. Sloan Management Review, 39(3):9-21, 1998.

[105] L. Finlay. "Outing" the researcher: The provenance, process, and practice of reflexivity. Qualitative Health Research, 12(4):531-545, 2002.

[106] R. Finnegan. Using documents. In R. Sapsford and V. Jupp, editors, Data Collection and Analysis, pages 138-151. Thousand Oaks, CA: Sage, 1996.

[107] U. Flick. An Introduction to Qualitative Research. Sage Publications, third edition, 2006.

[108] S. W. Floyd and B. Wooldridge. Path analysis of the relationship between competitive strategy, information technology, and financial performance. Journal of Management Information Systems, 7(1):47-64, 1990.

[109] G. Fox. Sourcing inside out: Penalties and incentives in outsourcing contracts, 2008. White Paper of Sourcingmag.com Practical advice for IT and business process outsourcing.

[110] J. Fulk and G. DeSanctis. Electronic communication for changing organizational forms. Organization Science, 6(4):337-349, 1995.

[111] H.-G. Gadamer. Philosophical Hermeneutics. University of California Press, 1977.

[112] J. R. Galbraith. Designing organizations: An executive briefing on strategy, structure, and process. Jossey-Bass, 1995.

[113] R. D. Galliers. On confronting some common myths of IS strategy discourse. In R. Mansell, C. Avgerou, and D. Quah, editors, The Oxford Handbook of Information and Communication Technologies, pages 225-243. Oxford University Press, New York, NY, USA, 2007.

[114] M. Galster, A. Eberlein, and M. Moussavi. Transition from requirements to architecture: A review and future perspective. In SNPD-SAWN '06: Proceedings of the Seventh ACIS International Conference on Software Engineering, Artificial Intelligence, Networking, and Parallel/Distributed Computing, pages 9-16, Washington, DC, USA, 2006. IEEE Computer Society.

[115] C. I. García Romero. El Modelo de Capacidad de Madurez y su Aplicación en Empresas Mexicanas de Software. BA thesis, Universidad de las Américas, Cholula, Puebla, Mexico, 2001. In Spanish.

[116] R. A. Giacalone and P. Rosenfeld. Impression Management in the Organization. Lawrence Erlbaum Associates, 1989.

[117] H. Gijlers, W. R. van Joolingen, T. de Jong, and B. H. A. M. van Hout-Wolters. Interaction between tool and talk: How instruction and tools support consensus building in collaborative inquiry-learning environments. Journal of Computer Assisted Learning, 25(3):252-267, 2009.

[118] J. Gordijn.Value-based requirements engineering: Exploring innovative e-commerce ideas. PhD thesis, Vrije Universiteit Amsterdam, The Netherlands, 2002. 


\section{BIBLIOGRAPHY}

[119] G. E. Gorman and P. Clayton. Qualitative research for the information professional. Facet Publishing, second edition, 2005.

[120] P. Gugler and J. H. Dunning. Technology based cross-border alliances. In R. Culpan, editor, Multinational Strategic Alliances, pages 123-165. International Business Press, 1993.

[121] R. Gulati and M. Gargiulo. Where do interorganizational networks come from? American Journal of Sociology, 104(5):1439-1493, 1999.

[122] S. Gunderson. A review of organizational factors and maturity measures for system safety analysis. Systems Engineering, 8(3):234-244, 2005.

[123] A. Halinen, A. Salmi, and V. Havila. From dyadic change to changing business networks: an analytical framework. Journal of Management Studies, 26(6):779$794,1999$.

[124] J. Hartman. Using focus groups to conduct business communication research. The Journal of Business Communication, 41(4):402-410, 2004.

[125] S. Hartung, B. H. Reich, and I. Benbasat. Information technology alignment in the canadian forces. Canadian Journal of Administrative Sciences, 17(4):285-302, 2000 .

[126] I. S. Hayes. Creating better service level metrics, 2007. White Paper of Clarity Consulting, Inc.

[127] J. C. Henderson. Plugging into strategic partnerships: The critical IS connection. Sloan Management Review, 31(3):7-18, 1990.

[128] J. C. Henderson and J. G. Sifonis. The value of strategic IS planning: understanding consistency, validity, and IS markets. MIS Quarterly, 12(2):187-200, 1988.

[129] J. C. Henderson and J. B. Thomas. Aligning business and information technology domains: Strategic planning in hospitals. Hospital and Health Services Administrative, 37(1):71-87, 1992.

[130] J. C. Henderson and H. Venkatraman. Strategic alignment: Leveraging information technology for transforming organizations. IBM Systems Journal, 32(1):472484, 1993.

[131] J. C. Henderson, N. Venkatraman, and S. Oldach. Aligning business-IT strategies. In J. Luftman, editor, Competing in the Information Age, pages 21-42. Oxford University Press, 1996.

[132] P. J. Henry, G. M. Hart, and D. W. Nance. Supervision topics as perceived by supervisors and supervisees. The Clinical Supervisor, 23(2):139-152, 2006.

[133] A. R. Hevner, S. T. March, J. Park, and S. Ram. Design science in information systems research. MIS Quarterly, 28(1):75-105, 2004.

[134] W. Higgins and K. T. Hallstrom. Standardization, globalization and rationalities of government. Organization, 14(5):685-704, 2007.

[135] J. A. Hipps. Trustworthiness and authenticity: Alternate ways to judge authentic assessments, 1993. Paper presented at the annual meeting of the American Educational Research Association.

[136] M. T. Holden and T. O'Toole. A quantitative exploration of communication's role in determining the governance of manufacturerretailer relationships. Industrial Marketing Management, 33(6):539-548, 2004.

[137] S. Holder. Settlement service standards: An inventory of work-in-progress and future steps, 2001. Ontario Council of Agencies Serving Immigrants.

[138] F. Hoque. The Alignment Effect. FT Press, 2002.

[139] J. Horward. LVO Inrichting v 1.1, 2008. In Dutch. 
[140] S. H. Houmb, V. N. L. Franqueira, and E. A. Engum. Estimating impact and frequency of risks to safety and mission critical systems using cvss. In ISSRE 2008 Supplemental Proceedings: 1st Workshop on Dependable Software Engineering, Seattle, US, IEEE CS Conference Proceedings, Washington, US, November 2008. IEEE Computer Society Press.

[141] R. H. Hoyle. Statistical strategies for small sample research. Thousand Oaks, CA: Sage, 1999.

[142] C. D. Huang and Q. Hu. Achieving IT-Business strategic alignment via enterprisewide implementation of balanced scorecards. Information Systems Management, 24(2):173-184, 2007.

[143] S.-H. Hung and E. S. Davidson. System support for dynamic optimization of application performance. In IWIA '98: Proceedings of the 1998 International Workshop on Innovative Architecture, pages 7-20, Los Alamitos, CA, USA, 1998. IEEE Computer Society.

[144] Information Systems Audit and Control Association (ISACA). COBIT: 4th Edition, 2006.

[145] J. E. Innes. Consensus building: Clarifications for the critics. Planning Theory, $3(1): 5-20,2004$.

[146] International Organization for Standardization. ISO 9001, Quality management systemsrequirements, 2000.

[147] International Organization for Standardization. ISO/IEC 17799:2005, 2005.

[148] P. Janssen. IT-service management volgens ITIL. Addison Wesley, 2003. In Dutch.

[149] G. Jiménez, N. Galeano, T. Nájera, J. M. Aguirre, C. Rodríguez, and A. Molina. Methodology for business model definition of collaborative networked organizations. In Collaborative Networks and Their Breeding Environments, pages 347354. Springer Boston, 2005.

[150] B. Johnson and L. B. Christensen. Educational Research: Quantitative, Qualitative, and Mixed Approaches. Allyn \& Bacon, second edition, 2004.

[151] R. B. Johnson. Examining the validity structure of qualitative research. Education, 118(1), 1997.

[152] M. L. Kaarst-Brown and D. Robey. More on myth, magic and metaphor: Cultural insights into the management of information technology in organizations. Information Technology \& People, 12(2):192-218, 1999.

[153] V. Kartseva. Designing Controls for Network Organizations: A Value-Based Approach. PhD thesis, Vrije Universiteit Amsterdam, Netherlands, 2008.

[154] G. S. Kearns and A. L. Lederer. The effect of strategic alignment on the use of ISbased resources for competitive advantage. The Journal of Strategic Information Systems, 9(4):265-293, 2000.

[155] G. S. Kearns and A. L. Lederer. A resource-based view of strategic IT alignment: How knowledge sharing creates competitive advantage. Decision Sciences, 34(1):1-29, 2003.

[156] P. G. W. Keen. Shaping the future: business design through information technology. Harvard Business School Press, Boston, MA, USA, 1991.

[157] P. G. W. Keen. Do you need an IT strategy? In J. Luftman, editor, Competing in the Information Age, pages 137-178. Oxford University Press, 1996.

[158] P. G. W. Keen. Achieving business benefits from ERP systems. In C. Ferran and R. Salim, editors, Enterprise Resource Planning for Global Economies: Managerial Issues and Challenges, pages 77-92. Idea Group Inc (IGI), 2008. 
[159] P. G. W. Keen and S. Qureshi. Organizational transformation through business models: A framework for business model design. In HICSS '06: Proceedings of the 39th Annual Hawaii International Conference on System Sciences, page 206.2, Washington, DC, USA, 2006.

[160] D. Kennedy. Thinking for oneself and with others. Analytic Teaching, 20(1):4045, 1999.

[161] Kenniscentrum. NORA 2.0: Samenhang en samenwerking binnen de elektronische overheid, 2006. In Dutch.

[162] G. Kerr and L. Simard. Powers, evaluation of the OASIS computerization project: Final Report, 2002. Retrieved October 15, 2008 from http://www.realworldsystems.net.

[163] B. Kitchenham, S. Pfleeger, L. Pickard, P. Jones, D. Hoaglin, K. Emam, and J. Rosenberg. Preliminary guidelines for empirical research in software engineering. IEEE Transactions on Software Engineering, 28(8):721-734, 2002.

[164] B. Kitchenham, L. Pickard, and S. L. Pfleeger. Case studies for method and tool evaluation. IEEE Software, 12(4):52-62, 1995.

[165] H. K. Klein and M. D. Myers. A set of principles for conducting and evaluating interpretive field studies in information systems. MIS Quarterly, 23(1):67-93, 1999.

[166] T. Knothe, K. Schneider, D. Böl, T. Kahl, S. Schuster, F. Lillehagen, J. Krogstie, and H. G. Solheim. Framework for the establishment and management methodology, 2005. Deliverable A.1.4.1, ATHENA, Integrated Project Contract Num. IST-507849.

[167] B. Konsynski and W. F. McFarlan. Information partnerships - shared data, shared scale. Harvard Business Review, 68(5):114-120, 1990.

[168] J. Kontio, L. Lehtola, and J. Bragge. Using the focus group method in software engineering: Obtaining practitioner and user experiences. In Proceedings of the 2004 International Symposium on Empirical Software Engineering (ISESE'04), pages 271-280. IEEE Computer Society, 2004.

[169] N. Korac-Kakabadse, A. Kouzmin, and A. Korac-Kakabadse. Information tech nology-enabled communication and organizational effectiveness. International Review of Public Administration, 5(1):368-377, 2000.

[170] D. Krackhardt. Assessing the political landscape: Structure, cognition, and power in organizations. Administrative Science Quarterly, 35(1):342-369, 1990.

[171] R. A. Krueger and M. A. Casey. Focus Groups: A Practical Guide for Applied Research. Thousand Oaks, CA: Sage Publications, 2000.

[172] S. Kumar and S. Craig. Dell, Inc.'s closed loop supply chain for computer assemby plants. Information Knowledge, Systems Management, 6(3):197-214, 2007.

[173] M. Lankhorst. ArchiMate language primer: Introduction to the ArchiMate language for enterprise architecture, 2005. Telematica Instituut. Publication Num. $\mathrm{TI} / \mathrm{RS} / 2004 / 024$.

[174] K. Larsor, N. Grudens-Schuck, and B. Lundy Allen. Can you call it a focus group?, May 2004. IOWA State University. Methodology brief.

[175] A. Lee and R. Baskerville. Generalizing generalizability in information systems research. Information Systems Research, 14(3):221-243, 2003.

[176] S. Lee and R. P. Leifer. A framework for linking the structure of information systems with organizational requirements for information sharing. Journal of Management Information Systems, 8(4):27-45, 1992. 
[177] Y. Lee, K. A. Kozar, and K. R. T. Larsen. The technology acceptance model: Past, present and future. Communications of the Association for Information Systems, 12(50):752-780, 2007.

[178] M. Leussink. Praktijkproeven voorbeeldproject WABO-ICT, 2008. In Dutch.

[179] Y. Levy and T. Ellis. Towards a framework of literature review process in support of information systems research. In Proceedings of the 2006 Informing Science and IT Education Joint Conference, pages 172-181, 2006.

[180] C. A. Lietz, C. L. Langer, and R. Furman. Establishing trustworthiness in qualitative research in social work: Implications from a study regarding spirituality. Qualitative Social Work, 5(4):441-458, 2006.

[181] Y. S. Lincoln and E. G. Guba. Naturalistic Inquiry. Sage Publications, sixth edition, 1985.

[182] J. Lipnack and J. Stamps. The networking book, people connecting with people. Routledge and Kegan Paul, 1986.

[183] J. Lipnack and J. Stamps. The teamNet factor: Bringing the power of boundary crossing into the heart of your business. Oliver Wight Publications, Inc., 1993.

[184] P. Liu. Unpacking the black box of IT capabilities. In Proceedings of the Third Midwest United States Association for Information Systems, pages 7-20. AIS, 2008.

[185] A. Lockamy and K. McCormack. The developmentof a supply chain management process maturity model using the concepts of business process orientation. Supply Chain Management, 9(4):272-278, 2004.

[186] K. O. Locker. The challenge of interdisciplinary research. Journal of Business Communication, 31(2):137-151, 1994.

[187] H. C. Lucas Jr. and J. Baroudi. The role of information technology in organization design. Journal of Management Information Systems, 10(4):9-23, 1994.

[188] J. N. Luftman. Competing in the Information Age. Oxford University Press, 1996.

[189] J. N. Luftman. Assessing IT-business alignment. Information Systems Management, 20(4):9-15, 2003.

[190] J. N. Luftman, P. R. Lewis, and S. H. Oldach. Transforming the enterprise: The alignment of business and information technology strategies. IBM Systems Journal, 32(1):198-221, 1993.

[191] J. N. Luftman, R. Papp, and T. Brier. Enablers and inhibitors of business-IT alignment. Communications of the AIS, 1(3es), 1999.

[192] R. Maes, V. Dirksen, and O. Truijens. Redefining business-IT alignment, 2000. University of Amsterdam. Working paper.

[193] A. Magalhaes, R. Araújo, and M. R. S. Borges. Designing collaborative processes. In Proceedings of the 8th Workshop on Business Process Modeling, Development and Support (BPMDS'O7) in the 19th International Conference on Advance Information Systems Engineering (CAISE'07), 2007.

[194] A. Magalhaes, C. Cappelli, F. Araujo Baiao, F. M. Santoro, and R. Araujo. Towards collaboration maturity in business processes: An exploratory study in oil production processes. Information Systems Management, 25(4):302-318, 2008.

[195] M. W. Maier, D. Emery, and R. Hilliard. Software architecture: Introducing IEEE standard 1471. Computer, 34(4):107-109, 2001.

[196] Y. Malhotra. Role of information technology in managing organizational change and organizational interdependence, 1993. Retrieved May 01, 2006 from http://www.brint.com/papers/change/.

[197] T. W. Malone and K. Crowston. The interdisciplinary study of coordination. ACM computing surveys, 26(1):87-120, 1994. 
[198] R. Marks and R. E. Hebner. Government activity to increase benefits from the global standards system. In SIIT, pages 183-190. IEEE, 2001.

[199] G. Martin, A. Pallotta-Cornick, G. Johnstone, and A. C. Goyos. A supervisory strategy to improve work performance for lower functioning retarded clients in a sheltered workshop. Journal of Applied Behavior Analysis, 13(1):183-190, 1980.

[200] J. A. McDermid. Requirements analysis: orthodoxy, fundamentalism and heresy. In M. Jaroticka and J. Goguen, editors, Requirements engineering: social and technical issues, pages 17-40. Academic Press Professional, Inc., San Diego, CA, USA, 1994.

[201] T. McGuinness. Markets and managerial hierarchies. In R. Clarke and T. McGuin ness, editors, The Economics of the Firm, pages 42-61. Basil Blackwell, Oxford, 1987. First published in Economica (4), 1937, pp.386-405.

[202] N. Melville, K. Kraemer, and V. Gurbaxani. Information technology and organizational performance: An integral model of IT business value. MIS Quarterly, 28(2):283-322, 2004.

[203] H. B. Memduhog, I. Aydin, K. Yilmaz, S. Gngr, and E. Og. The process of supervision in the turkish educational system: purpose, structure, operation. Asia Pacific Education Review, 8(1):56-70, 2009.

[204] META Group. Architecture maturity audit: Part 1. META Practice, 4(4), 2000.

[205] H. Middel, J. Gieskes, and O. Fisscher. Driving collaborative improvement processes. Production Planning \& Control, 16(4):368-377, 2005.

[206] H. Mintzberg. Structure in Fives: Designing Effective Organizations. Prentice Hall, Englewood Cliffs, NJ., second edition, 1993.

[207] K. K. Möller and A. Halinen. Business relationships and networks: Managerial challenge of network era. Industrial Marketing Management, 28(5):413-427, 1999.

[208] A. Montoro Sánchez. El desarrollo de redes organizativas. fundamentos teóricos y enfoques metodológicos. Cuadernos de Estudios Empresariales de la Universidad Complutense de Madrid, 10:185-204, 2000. In Spanish.

[209] D. L. Moody. Dealing with complexity: A practical method for representing large entity relationships models. $\mathrm{PhD}$ thesis, University of Melbourne, Australia, 2001.

[210] K. W. Moody. New meaning to IT alignment. Information Systems Management, 20(4):30-35, 2003.

[211] M. Mora, O. Gelman, D. Paradice, and F. Cervantes. The case of conceptual research in information systems. In Proceedings of the International Conference on Information Resources Management (Conf-IRM 2008), pages 1-10, Niagara Falls, Ontario, Canada, 2008.

[212] D. L. Morgan. Successful Focus Groups: Advancing the State of the Art. Sage Publications, 1993.

[213] D. L. Morgan. Focus Group as Qualitative Research method. Sage Publications, second edition, 1997.

[214] F. Moulaert and K. Cabaret. Planning, networks and power relations: is democratic planning under capitalism possible? Planning Theory, 5(1):51-70, 2006.

[215] P. N. Murray. Focus groups are valid when done right. Marketing News, 40(14):21 \& 25, September 2006.

[216] N. Condori Fernández and O. Pastor. An empirical study on the likelihood of adoption in practice of a size measurement procedure for requirements specification. In QSIC '06: Proceedings of the Sixth International Conference on Quality Software, pages 133-140, Washington, DC, USA, 2006. IEEE Computer Society. [217] D. S. Newman. Class-based reengineering. Object magazine, 6:68-83, 1996. 
[218] F. Niessink. Perspectives on improving software maintenance. PhD thesis, Vrije Universiteit Amsterdam, Amsterdam, The Netherlands, 2000.

[219] C. Nikendei, A. Zeuch, P. Dieckmann, C. Roth, S. Schäfer, M. Völkl, D. Schellberg, W. Herzog, and J. Jünger. Role-playing for more realistic technical skills training. Medical Teacher, 27(2):122-126, 2005.

[220] L. R. Nolan. Managing the crisis in data processing. Harvard Business Review, 57(2):115-126, 1979.

[221] R. Normann and R. Ramírez. From value chain to value constellation: Designing interactive strategy. Harvard Business Review, 71(4):65-77, 1993.

[222] C. Okoli and S. D. Pawlowski. The delphi method as a research tool: an example design considerations and applications. Information and Management, 42(1):1529, 2004.

[223] Ontario Council of Agencies Serving Immigrants. Models of settlement service workshop, co-located with the OCASI Annual Conference, 2000. Retrieved October 15, 2008 from http://atwork.settlement.org/downloads/atwork/Models_ of_Settlement_Service.pdf.

[224] A. J. Onwuegbuzie and N. L. Leech. Validity and qualitative research: An oxymoron? Quality and Quantity, 41(2):233-249, 2006.

[225] Oracle Corp. Ensuring value in application investment - primavera portfolio management, 2009. Oracle Data Sheet.

[226] D. K. Padgett. Qualitative Methods in Social Work Research. Sage Publications, second edition, 2008.

[227] G. Paré and J. Elam. Using case study research to build theories of IT implementation. In A. Lee, J. Liebunau, and J. DeGross, editors, Information Systems and Qualitative Research, pages 70-100. Chapman and Hall, London, U.K., 1997.

[228] R. Pawson. Theorizing the interview. British Journal of Sociology, 47(2):295-314, 1996.

[229] R. Pawson, T. Greenhalgh, G. Harvey, and K. Walshe. Realist synthesis: An introduction, 2004. ESRC Research Methods Programme. University of Manchester. RMP Methods Paper No.2.

[230] R. Pawson and N. Tilley. Realistic Evaluation. Sage Publications, 1997.

[231] D. Peak and C. S. Guynes. The IT alignment planning process. Journal of Computer Information Systems, 44(1):9-15, 2003.

[232] J. L. Pershing. Using document analysis in analyzing and evaluating performance. Performance Improvement, 41(1):36-42, 2007.

[233] S. Petter, W. DeLone, and E. McLean. Measuring information systems success: Models, dimensions, measures, and interrelationships. European Journal of Information Systems, 17(3):236-263, 2008.

[234] J. Pfeffer and G. R. Salancik. The external control of organizations: A resource dependence perspective. Stanford business classics. Stanford Business Books, New York, NY., 2003. Originally published: New York : Harper \& Row, 1978.

[235] S. L. Pfleeger. Software engineering: Theory and practice. Prentice Hall, second edition, 2001.

[236] G. P. Pisano and R. Verganti. Which kind of collaboration is right for you? Harvard Business Review, Dec 2008.

[237] R. Poels. Beïnloeden en meten van businessIT alignment. PhD thesis, Vrije Universiteit Amsterdam, Netherlands, 2006. In Dutch.

[238] M. E. Porter. Competitive Advantage: Creating and Sustaining Superior Performance. Free Press, New York, 2004. Originally published: New York: Free Press, 1985. 


\section{BIBLIOGRAPHY}

[239] A. Powell, G. Piccoli, and B. Ives. Virtual teams: A review of current literature and directions for future research. SIGMIS Database, 35(1):6-36, 2004.

[240] R. R. Powell. Basic Research Methods for Librarians. Greenwood Publishing Group, third edition, 1997.

[241] T. C. Powell. Organizational alignment as competitive advantage. Strategic Management Journal, 13(2):119-134, 1992.

[242] T. C. Powell and A. Dent-Micallef. Information technology as competitive advantage: the role of human, business, and technology resources. Strategic Management Journal, 8(5):375-405, 1999.

[243] A. Prasad. The contest over meaning: Hermeneutics as an interpretive methodology for understanding texts. Organizational Research Methods, 5(1):12-33, 2002.

[244] Provincie Overijssel. Samenhang voor samenwerking: Een project start architectuur omgevingsvergunning voor het voorbeeldproject overijssel, 2007. In Dutch.

[245] Provincie Overijssel. Met het oog op morgen!, March 2008. WABO-bijeenkomt Provincie Overijssel. In Dutch.

[246] V. Prybutok, R. Richards, and R. Cutshall. The significance of information and analysis as a component of a leadership model based on Malcolm Baldrige National Quality Award. Journal of Computer Information Systems, 41(4):52-56, 2001.

[247] P. M. Pyett. Validation of qualitative research in the "Real World". Qualitative Health Research,, 13(8):1170-1179, 2003.

[248] H. G. Rainey and B. Bozeman. Comparing public and private organizations: Empirical research and the power of the a priori. Journal of Public Administration Research Theory, 10(2):447-470, 2000.

[249] A. Ramakumar and B. Cooper. Process standardization proves profitable. Quality, 43(2):42-45, 2004.

[250] N. Ramasubbu, M. Krishnan, and P. Kompalli. A process maturity framework for managing distributed development. IEEE Software, 22(3):80-86, 2005.

[251] V. Ramesh, R. L. Glass, and I. Vessey. Research in computer science: An empirical study. Journal of systems and software, 70(1):165-176, 2004.

[252] M. Rappa. Business models on the web, 2006. Reading of the course: Managing the Digital Enterprise of the North Carolina State University, USA.

[253] B. H. Reich and I. Benbasat. Development of measures to investigate the linkage between business and information technology objectives. MIS Quarterly, 20(1):55-81, 1996.

[254] B. H. Reich and I. Benbasat. Factors that influence the social dimension of alignment between business and information technology objectives. MIS Quarterly, 24(1):81-113, 2000.

[255] A. M. Riege. Validity and reliability tests in case study research: a literature review with "hands-on" applications for each research phase. Qualitative Market Research: An International Journal, 6(2):75-86, 2003.

[256] K. Riemer and S. Klein. Network management framework: Organizational perspectives of technology enabled inter-firm collaboration. In S. Klein and A. Poulymenakou, editors, Managing Dynamic Networks, pages 17-66. Springer Berlin Heidelberg, 2006.

[257] G. Riempp and S. Gieffers-Ankel. Application portfolio management: A decisionoriented view of enterprise architecture. Information Systems and e-Business Management, 5(4):359-378, 2007. 


\section{BIBLIOGRAPHY}

[258] C. Rolland, S. Nurcan, and G. Grosz. A decision-making pattern for guiding the enterprise knowledge development process. Information and Software Technology, 42(5):313-331, 2000.

[259] D. Rondinelli, B. Rosen, and I. Drori. The struggle for strategic alignment in multinational corporations: Managing readjustment during global expansion. European Management Journal, 19(4):404-416, 2001.

[260] J. Ross. Creating a strategic IT architecture competency: Learning in stages. MISQ Executive, 2(1):31-43, 2003.

[261] C. Rossignoli. The contribution of transaction cost theory and other networkoriented techniques to digital markets. Information Systems and e-Business Management, 7(1):57-79, 2009.

[262] R. Sabherwal and Y. E. Chan. Alignment between business and IS strategies: A study of prospectors, analyzers and defenders. IS Research, 12(1):11-33, 2001.

[263] L. Salter and A. Hearn, editors. Outside the lines: Issues in interdisciplinary research. McGill-Queen's University Press, 1996.

[264] Sanaghan Group. Crafting a supervision process. Concept paper available at: http://www.thesanaghangroup.com/concept_papers.php.

[265] A. Sanchez Ortiz. Testing a model of the relationships among organizational performance, IT-business alignment, and IT governance. PhD thesis, University of North Texas, Denton, Texas, USA, 2003.

[266] R. Santana Tapia. IT process architectures for enterprises development: A survey from a maturity model perspective. Technical Report TR-CTIT-06-04, University of Twente, Enschede, The Netherlands, January 2006.

[267] R. Santana Tapia. What is a networked business? Technical Report TR-CTIT06-23a, University of Twente, Enschede, The Netherlands, 2006.

[268] R. Santana Tapia. Converging on business-IT alignment best practices: Lessons learned from a Dutch cross-governmental partnership. In Proceedings of the 15th International Conference on Concurrent Enterprising (ICE 2009) "Collaborative Innovation: Emerging Technologies, Environments and Communities", 2009. 2224 Jun 2009, Leiden, The Netherlands.

[269] R. Santana Tapia. Coordination and partnering structure are vital domains in collaborative business-IT alignment: Elaborating on the ICoNOs MM. In Proceedings of the 4th IEEE/IFIP International Workshop on Business-Driven IT Management (BDIM 2009), pages 73-74, Long Island, New York, USA, 2009. IEEE Computer Society Press.

[270] R. Santana Tapia. ICoNOs MM: The IT-enabled collaborative networked organizations maturity model. In Leveraging knowledge for innovation in Collaborative Networks, 10th IFIP Working Conference on Virtual Enterprises, Lecture Notes in Computer Science, Thessaloniki, Greece, 2009. Springer Verlag.

[271] R. Santana Tapia, M. Daneva, and P. van Eck. Developing an inter-enterprise alignment maturity model: Research challenges and solutions. In C. Rolland, O. Pastor, and J.-L. Cavarero, editors, Proceedings of the 1st Int. Conf. on Research Challenges on Information Science (RCIS'07), pages 51-59, Ouarzazate, Morocco, 2007.

[272] R. Santana Tapia, M. Daneva, and P. van Eck. Developing an inter-enterprise alignment maturity model: Research challenges and solutions. Technical Report TR-CTIT-07-29, Centre for Telematics and Information Technology, University of Twente, Enschede, May 2007. 


\section{BIBLIOGRAPHY}

[273] R. Santana Tapia, M. Daneva, and P. van Eck. Validating adequacy and suitability of business-IT alignment criteria in an inter-enterprise maturity model. In Proceedings of the Eleventh IEEE International EDOC Enterprise Computing Conference (EDOC'O7), pages 202-213, Los Alamitos, October 2007. IEEE Computer Society Press.

[274] R. Santana Tapia, M. Daneva, P. van Eck, N. Castro Cárdenas, and L. van Oene. Business-IT alignment domains and principles for networked organizations: A qualitative multiple case study. In On the Move to Meaningful Internet Services: OTM 2008 Workshops Proceedings, volume 5333/2008 of Lecture Notes in Computer Science, pages 241-252. Springer Verlag, 2008.

[275] R. Santana Tapia, M. Daneva, P. van Eck, and R. Wieringa. Towards a businessIT alignment maturity model for collaborative networked organizations. In Proceedings of the International Workshop on Enterprise Interoperability (IWEI 2008), CTIT Workshop Proceedings Series WP08-05, pages 70-81, Enschede, 2008. Centre for Telematics and Information Technology.

[276] R. Santana Tapia, P. van Eck, and M. Daneva. Validating the domains of an inter-organizational business-IT alignment assessment instrument: A case study. Technical Report TR-CTIT-08-53, Enschede, August 2008.

[277] R. Santana Tapia and L. van Oene. Some empirical evidence on business-IT alignment processes in the public sector: A case study report. Technical Report TR-CTIT-08-46, Enschede, June 2008.

[278] R. Santana Tapia and N. Zarvić. Value-based partnering structure design for networked businesses: A multi-method approach. In Proceedings of 21st Bled Conference "eCollaboration" Overcoming Boundaries through Multi-Channel Interaction, pages 263-276, Bled, Slovenia, 2008. Kranj: Moderna organizacija.

[279] J. Scanlon. A Capital One exec addresses alignment. Journal of Information Technology, 1(22), 2003.

[280] J. Schekkerman. Extended Enterprise Architecture Maturity Model Support Guide v2.0. Institute for Enterprise Architecture Developments, 2006.

[281] T. Schijvenaars and M. Keijers. SOA is wondermiddel voor gemeenten. Computable, February 2008. In Dutch.

[282] C. Seale. Quality in qualitative research. Qualitative Inquiry, 5(4):465-478, 1999.

[283] S. H. Seggie, D. Kim, and S. Cavusgil. Do supply chain IT alignment and supply chain interfirm system integration impact upon brand equity and firm performance? Journal of Business Research, 59(8):887-895, 2006.

[284] A. Senn. Re aling: Tackling business and IT alignment, 2004. CIO Advertising Supplement, Deloitte Development LLC.

[285] W. R. Shadish, T. D. Cook, and D. T. Campbell. Experimental and quasiexperimental designs for generalized causal inference. Houghton Mifflin, second edition, 2001.

[286] S. M. Shafer, H. J. Smith, and J. C. Linder. The power of business models. Business Horizons, 48(3):199-207, 2005.

[287] U. Shakir. Re-visioning the newcomer settlement support system by ISPR consortium, presented in the OCASI Annual Conference, 2000. Retrieved October 15, 2008 from http://atwork.settlement.org/downloads/newcomer_settle ment_support_system.pdf.

[288] D. Shaw. Bibliographic database searching by graduate students in language and literature: Search strategies, system interfaces, and relevance judgements. Library E Information Science Research, 17(4):327-345, 1995. 
[289] Y. Simmons. Using focus groups as an applied research tool. Howe's Now, 6(2), Apr 2000.

[290] C. C. Snow and J. B. Thomas. Field research methods in strategic management: Contributions to theory building and testing. Journal of management studies, 31(4):457-480, 1995.

[291] M. M. Snyder, J. Osland, and L. Hunter. Public and private organizations in Latin America: a comparison of reward preferences. International Journal of Public Sector Management, 9(2):15-27, 1996.

[292] I. Sommerville and P. Sawyer. Requirements Engineering: A good practice guide. John Wiley \& Sons, 1997.

[293] M. W. A. Steen, M. M. Lankhorst, and R. G. van de Wetering. Modelling networked enterprises. In Proceedings of the Sixth International Enterprose Distributed Object Computing (EDOC'02), pages 109-119, Washington, DC, USA, 2002. IEEE Computer Society.

[294] C. Stenbacka. Qualitative research requires quality concepts of its own. Management Decision, 39(7):551-556, 2001.

[295] D. Straub, S. And, and R. Evaristo. Normative standards for IS research. Data base, 25(1):21-34, 1994.

[296] P. P. Tallon and K. L. Kraemer. Investigating the relationship between strategic alignment and IT business value: The discovery of a paradox. In N. Shin, editor, Creating business value with information technology: Challenges and solutions, pages 1-22. IGI Publishing, Hershey, PA, USA, 2003.

[297] D. Tapscott. Believe the hypernet. Director, 54:48, 2001.

[298] D. Tapscott, D. Ticoll, and A. Lowy. Digital Capital - Harnessing the Power of Business Webs. Nicholas Brealy Publishing, 2000.

[299] F. M. Téllez. Solving the size estimation problem in ERP project context: The eEPC-COSMIC approach. Master thesis, University of Twente, Enschede, The Netherlands, 2009.

[300] T. S. H. Teo and W. R. King. Integration between business planning and information systems planning: An evolutionary-contingency perspective. Journal of Management Information Systems, 14(1):185-214, 1997.

[301] T. M. Teodorescu and C. Binder. Getting to the bottom line: Competence is what matters. Performance Improvement, 43(8):8-12, 2007.

[302] The Open Group. The Open Group Architecture Framework TOGAF -2007 edition (Incorporing 8.1.1). Van Haren Publishing, 2007.

[303] J. B. Thomas and R. Dewitt. Strategic alignment research and practice: A review and research agenda. In J. Luftman, editor, Competing in the Information Age, pages 385-403. Oxford University Press, 1996.

[304] J. D. Thompson. Organization in Action. McGraw-Hill, 1967.

[305] E. M. Trauth, editor. Qualitative research in IS: Issues and trends. Idea Group Publishing, Hershey, PA, USA, 2001.

[306] H. Tsoukas. Creating organizational knowledge dialogically: an outline of a theory. In T. Rickards, M. A. Runco, and S. Moger, editors, The Routledge Companion to Creativity, pages 160-176. Taylor \& Francis, 2008.

[307] S. H. Turner. So, you don't like focus groups anymore? Quirks Marketing Research Review, May 2006. Retrieved January 26, 2008 from http://www.fieldwork. com/turner_0506.pdf.

[308] B. Vagadia. Contract termination procedures. In Outsourcing to India - A Legal Handbook, pages 111-1115. Springer Berlin Heidelberg, 2007. 


\section{BIBLIOGRAPHY}

[309] A. H. van de Ven and M. S. Poole. Alternative approaches for studying organizational change. Organization Studies, 26(9):1377-1404, 2005.

[310] M. van den Berg and M. van Steenbergen. DYA: Stap voor stap naar professionele enterprise-architectuur. Ten Hagen \& Stam uitgevers, 2004. In Dutch.

[311] R. van der Pols. ASL: A framework for application management. Van Haren Publishing, 2004.

[312] B. van der Raadt, J. F. Hoorn, and H. van Vliet. Alignment and maturity are siblings in architecture assessment. In CAISE '05: Proceedings of the 17th International Conference on Advanced Information Systems Engineering, volume 3520/2005 of LNCS, pages 357-371, Porto, Portugal, 2005. Springer.

[313] B. van der Raadt, J. Soetendal, M. Perdeck, and H. van Vliet. Polyphony in architecture. In ICSE'04: Proceedings 26th International Conference on Software Engineering, pages 533-542, Washington, DC, USA, 2004. IEEE Computer Society.

[314] H. van der Zee, P. Laagland, and B. Hafkenscheid. Architectuur als Managementinstrument: Beheersing en Besturing van Complexiteit in het Netwerktijdperk. Ten Hagen Stam Uitgevers, 2001. In Dutch.

[315] J. T. M. van der Zee and B. de Jong. Alignment is not enough: integrating business and information technology management with the balanced business scorecard. Journal of Management Information Systems, 16(2):137-156, 1999.

[316] P. van Eck, H. Blanken, and R. Wieringa. Project GRAAL: towards operational architecture alignment. International Journal of Cooperative Information Systems, 13(3):235-255, Sep 2004.

[317] E. van Heck and P. Vervest. Smart business networks: how the network wins. Communications of the ACM, 50(6):28-37, 2007.

[318] R. van Hooff, P. Weghorst, and D. Verhoef. Ketenbesturing. Tijdschrift voor informatie en management (TIEM), (19):4-9, May 2007. In Dutch.

[319] S. Veenman. The training of coaching skills: an implementation study. Educational Studies, 21(3):415-431, 1995.

[320] N. Venkatraman. The concept of fit in strategy research: Toward verbal and statistical correspondence. The Academy of Management Review, 14(3):423-444, 1989.

[321] D. Verdon and G. McGraw. Risk analysis in software design. Security ES Privacy, IEEE, 2(4):79-84, Jul-Aug. 2004.

[322] B. von Halle and L. Goldberg. The business rule revolution: Running business the right way. Happy About, 2006.

[323] VROM Advies- en Expertdienst (ICT). Startarchitectuur landelijke voorziening omgevingsvergunning, 2007. In Dutch.

[324] H.-T. Wagner, T. Weitzel, and W. Koenig. Modeling the impact of alignment routines on it performance: An approach to making the resource based view explicit. In HICSS '05: Proceedings of the 38th Annual Hawaii International Conference on System Sciences, pages 230b-230b, Kona, Hawaii, USA, 2005.

[325] G. Walsham. Interpretative case studies in IS research: Nature and method. European Journal of Information Systems, 4(2):74-81, 1995.

[326] C. L. Wang and P. K. Ahmed. The informal structure: Hidden energies within the organization. Working paper WP008/02, University of Wolverhampton, Telford, Shropshire, UK, 2002.

[327] J. Ward. A portoflio approach to evaluating information systems investments and setting priorities. In L. Willcocks, editor, Information management: The 
evaluation of information systems investments, pages 81-97. Chapman \& Hall, 1994.

[328] J. Ward and E. Daniel. Benefits Management: Delivering Value from IS $\&$ IT Investments. John Wiley \& Sons, Ltd., 2006.

[329] J. Ward, P. Taylor, and P. Bond. Evaluation and realisation of IS/IT benefits: An empirical study of current practice. European Journal of Information Systems, 4(4):214-225, 1996.

[330] P. Webb and C. Pollard. Demystifying a hermeneutic approach to IS research. Australasian Journal of Information systems, 13(2):31-48, 2006.

[331] K. E. Weick. Sensemaking in organizations. Sage Publications, 1995.

[332] H. Weigand, F. van der Poll, and A. de Moor. Coordination through communication. In Proceedings of the 8th International Working Conference on the Language-Action Perspective on Communication Modelling (LAP 2003), pages 115-134, 2003.

[333] E. Wenger. Communities of practice: Learning, meaning, and identity. Cambridge University Press, 1999.

[334] R. Wieringa. Requirements researchers: Are we really doing research?. Requirements Engineering, 10(4):304-306, 2005.

[335] R. Wieringa. Operational business-IT alignment in value webs. In R. K. et al., editor, Proceedings of UNISCON 2008, pages 371-378. Springer-Verlag, 2008.

[336] R. Wieringa, H. Akkermans, J. Gordijn, and P. van Eck. Value-Based Business-IT Alignment (VITAL) Research proposal. University of Twente \& Vrije Universiteit Amsterdam, 2004.

[337] R. Wieringa and J. M. G. Heerkens. The methodological soundness of requirements engineering papers: A conceptual framework and two case studies. Requirements Engineering, 11(4):295-307, 2006.

[338] R. T. Wigand. Electronic commerce: Definition, theory, and context. The Information Society, 13(1):1-16, 1997.

[339] R. T. Wigand, A. Picot, and R. Reichwald. Information, organization and management: Expanding markets and corporate boundaries, 1997.

[340] O. E. Williamson. Markets and hierarchies, analysis and antitrust implications: a study in the economics of internal organization. The Free Press, first edition, 1975.

[341] O. E. Williamson. The Economic Institutions of Capitalism: Firms, Markets, Relational Contracting. Free Press, 1985.

[342] O. E. Williamson. Transaction cost economics. In R. Schmalensee and R. D. Willig, editors, Handbook of Industrial Organization, pages 135-182. New York: North Holland, 1989.

[343] O. E. Williamson. Comparative economic organization: The analysis of discrete structural alternatives. Administrative Science Quarterly, 36(2):269-296, 1991.

[344] O. E. Williamson. The Mechanisms of Governance. New York: Oxford University Press, 1996.

[345] G. Winter. A comparative discussion of the notion of 'validity' in qualitative and quantitative research. The Qualitative Report, 4(3 \& 4), 2000. Retrieved January 26, 2008 from http://www.nova.edu/ssss/QR/QR4-3/winter.html.

[346] M. Witzel. IEBM dictionary of business and management. International Thomson Business Press, 1998.

[347] H. Wohlwend and S. Rosenbaum. Schlumberger's software improvement program. IEEE Transactions on Software Engineering, 20(11):833-839, 1994. 


\section{BIBLIOGRAPHY}

[348] R. K. Yin. Case study research: Design and methods. Applied Social Research Methods Series; vol. 5. Sage Publications, third edition, 2003.

[349] J.-H. Yu, H.-S. Lee, and W. Kim. Evaluation model for information systems benefits in construction management processes. Journal of Construction Engineering and Management, 132(10):1114-1121, 2006.

[350] R. Zarnekow, A. Hochstein, and W. Brenner. Serviceorientiertes IT-Management. Springer-Verlag, Berlin, 2005. In German.

[351] N. Zarvic and R. Wieringa. An integrated enterprise architecture framework for business-IT alignment. In CAISE '06: Proceedings of Workshops and Doctoral Contsortium of the 18th International Conference on Advanced Information Systems Engineering, pages 262-270, Luxembourg, Grand Duchy of Luxembourg, 2006. Namur University Press.

[352] N. Zarvic, R. Wieringa, and P. van Eck. Checking the alignment of value-based business models and IT functionality. Technical Report TR-CTIT-07-78, Enschede, November 2007.

[353] R. A. Zeller. Focus group research on sensitive topics: Setting the agenda without setting the agenda. In D. L. Morgan, editor, Successful focus groups: Advancing the state of the art, pages 167-183. Sage Publications, 1993.

[354] H. Zhou, D. A. Collier, and D. D. Wilson. The relationship of strategic business alignment and enterprise information management in achieving better business performance. Enterprise Information Systems, 2(2):201-220, 2008. 



\section{Samenvatting}

Vraagstukken zoals het identificeren van manieren om kosten te controleren, kwaliteit te verbeteren, doeltreffendheid te verhogen, en risico te beheren zijn in toenemende mate belangrijk voor organisaties geworden aangezien zij meer en meer druk ondervinden om hun maximaal concurrentievermogen te bereiken en te handhaven. Business-IT alignment (B-ITa) wordt gezien als een oplossing voor deze vraagstukken. Het richten van IT op de business blijft één van de hoogste prioriteiten voor zowel bedrijfsvaklieden als onderzoekers. Interesse in B-ITa wordt bevorderd door casussen van organisaties die met succes hun IT hebben gericht om concurrentievoordeel te bereiken en om vaardigheden te verbeteren.

$\mathrm{Er}$ is een aanzienlijke literatuur op B-ITa in single organisaties. Binnen dit brede werkingsgebied van literatuur, hebben een aantal auteurs benadrukt B-ITa te beoordelen om B-ITa verbeteringsacties te plannen. Deze auteurs hebben volwassenheidsmodellen $\left(\mathrm{MMs}^{1}\right)$ ontwikkeld. MMs beschrijven de ontwikkeling van een specifiek domein in tijd. Gebaseerd op volwassenheidsbeoordelingen, weten de organisaties de mate waarin de processen in dergelijke domeinen voorspelbaar zijn. Namelijk kunnen de organisaties zich van bewust zijn of een specifiek gebied voldoende wordt geraffineerd en gedocumenteerd zodat de activiteiten op dergelijk gebied nu het potentieel hebben om hun gewenste resultaten te bereiken.

Nochtans, is B-ITa in netwerkorganisaties $\left(\mathrm{CNOs}^{2}\right)$ nauwelijks bestudeerd. Maar toch is het belangrijk omdat betere B-ITa een efficiënter gebruik van IT in CNOs met zich meebrengt. Dit ondersteunt de integratie van informatiesystemen en processen over organisatorische grenzen. CNOs vormen de kern van een nieuwe discipline die zich concentreert op de structuur, het gedrag, en de dynamica van netwerken van onafhankelijke organisaties die gebruik van IT maken om samen te werken om zo gemeenschappelijke doelstellingen beter te bereiken.

Ondanks de efficiënte toepassing van huidige B-ITa MMs voor single organisaties, is er geen MM die specifiek gericht is op de processen die nodig zijn om alignment tussen business en IT in CNOs te bereiken. Tot zo ver ging onze kennis op het tijdstip van het schrijven van dit proefschrift. Hierop introduceren we in dit proefschrift het ICoNOs MM: een MM om B-ITa in CNOs te beoordelen. Het ICoNOs MM stelt een reeks van aanbevelingen - b.v., cordinatiemechanismen, implementatiestrategien en organisatorische veranderingen, in de vorm van procesgebieden, specifieke doelstellingen en praktijken. Door zijn volwassenheidsniveaus, stelt het ICoNOs MM verbeteringsroutes voor die al domeinen die het belangrijkst zijn voor het bereiken van alignment in CNOs. Wij geloven dat het bereiken van B-ITa in CNOs complexer is dan in single organisaties. Dat is omdat $\mathrm{B}-\mathrm{ITa}$ in $\mathrm{CNO}$ settings wordt gedreven door doelstellingen van verschillende onafhankelijke organisaties in het algemeen zonder gecentraliseerde besluitvorming.

Door dit proefschrift, stellen wij de resultaten voor van vier literatuuronderzoeken, één focus groep, en zes case studies. Gebaseerd op deze onderzoekactiviteiten, ontwierpen en valideerden wij het ICoNOs MM en al zijn componenten.

\footnotetext{
${ }^{1}$ Afkorting in Engels voor Maturity Models

${ }^{2}$ Afkorting in Engels voor Collaborative Networked Organizations
} 



\section{Resumen}

Cuestiones tales como la identificación de maneras para controlar costes, para mejorar calidad, para aumentar eficacia, y para manejar riesgos, han llegado a ser cada vez más importantes en organizaciones que hacen frente a presiones externas para ganar y mantener su posición competitiva. La alineación del negocio con las tecnologías de información (Business-IT alignment, o 'B-ITa' por su abreviación en Inglés) es reconocida como una solución a estas cuestiones. Alinear las tecnologías de información con el negocio sigue siendo una de las prioridades para empresarios e investigadores. El interés en B-ITa es estimulado por algunos casos de organizaciones que han alineado con éxito sus tecnologías de información para conseguir ventaja competitiva y para mejorar su funcionamiento.

Hay una amplia gama de literatura sobre el fenómeno B-ITa en organizaciones individuales. Dentro de esta literatura, varios autores consideran que evaluar BITa es importante para planear acciones dirigidas a mejorar la B-ITa misma. Estos autores han desarrollado modelos de madurez (MMs) para apoyar dicha evaluación. Los MMs describen el desarrollo de un dominio específico en un cierto período. De acuerdo con evaluaciones de madurez, organizaciones saben en que grado procesos en tales dominios son fiables. Es decir, las organizaciones pueden ser conscientes de si un área específica está refinada y documentada suficientemente de modo que las actividades en tal área tienen el potencial para alcanzar los resultados deseados.

Sin embargo, B-ITa en redes de organizaciones (Collaborative Networked Organizations, o 'CNOs' por su abreviación en Inglés) es subestudiada. Este problema, empero, es importante porque una B-ITa mejorada exige un uso más eficiente de las tecnologías de información en CNOs. Esto ayuda a integrar sistemas de información con procesos a través de fronteras organizacionales. CNOs son la base de una nueva disciplina que se centra en la estructura, el comportamiento, y la dinámica de redes de organizaciones independientes que colaboran usando tecnologías de información para alcanzar objetivos comunes de una mejor manera.

Sin subestimar la utilidad de MMs actuales al evaluar B-ITa en organizaciones individuales, en nuestro conocimiento a la hora de escribir esta tesis doctoral, no hay MMs que específicamente traten los procesos necesarios para alcanzar la B-ITa en CNOs. En respuesta, nosotros desarrollamos el ICoNOs MM, el cual presenta una colección de recomendaciones (v.gr., mecanismos de coordinación, estrategias de implementación y cambios organizacionales) en la forma de áreas de proceso, metas específicas y prácticas. A través de sus niveles de madurez, el ICoNOs MM proporciona rutas para mejorar esos dominios que son los más importantes para alcanzar la B-ITa en CNOs. Nosotros creemos que alcanzar la B-ITa en redes de organizaciones es más complejo que en organizaciones individuals porque B-ITa en CNOs es afectada por metas de diversas organizaciones independientes (comúnmente sin procedimientos de toma de decisión centralizados).

A través de esta tesis doctoral, presentamos los resultados de cuatro revisiones de literatura, de un grupo focal, y de seis casos de estudio. De acuerdo con estas actividades de investigación conceptual y empírica, diseñamos y validamos el ICoNOs MM y los componentes que le conforman. 



\section{SIKS Dissertation Series}

1998

1998-1 Johan van den Akker (CWI)

DEGAS - An Active, Temporal Database of Autonomous Objects

1998-2 Floris Wiesman (UM)

Information Retrieval by Graphically Browsing Meta-Information

1998-3 Ans Steuten (TUD)

A Contribution to the Linguistic Analysis of Business Conversations

within the Language/Action Perspective

1998-4 Dennis Breuker (UM)

Memory versus Search in Games

1998-5 E.W.Oskamp (RUL)

Computerondersteuning bij Straftoemeting

1999

1999-1 Mark Sloof (VU)

Physiology of Quality Change Modelling;

Automated modelling of Quality Change of Agricultural Products

1999-2 Rob Potharst (EUR)

Classification Using Decision Trees and Neural Nets

1999-3 Don Beal (UM)

The Nature of Minimax Search

1999-4 Jacques Penders (UM)

The practical Art of Moving Physical Objects

1999-5 Aldo de Moor (KUB)

Empowering Communities: A Method for the Legitimate User-Driven

Specification of Network Information Systems

1999-6 Niek J.E. Wijngaards (VU)

Re-design of Compositional Systems

1999-7 David Spelt (UT)

Verification Support for Object Database Design

1999-8 Jacques H.J. Lenting (UM)

Informed Gambling: Conception and Analysis of a Multi-Agent

Mechanism for Discrete Reallocation.

2000

2000-1 Frank Niessink (VU)

Perspectives on Improving Software Maintenance

2000-2 Koen Holtman (TUE)

Prototyping of CMS Storage Management

2000-3 Carolien M.T. Metselaar (UVA)

Sociaal-Organisatorische Gevolgen van Kennistechnologie;

een Procesbenadering en Actorperspectief. 


\section{SIKS DISSERTATION SERIES}

2000-4 Geert de Haan (VU)

ETAG, A Formal Model of Competence Knowledge for User Interface Design

2000-5 Ruud van der Pol (UM)

Knowledge-based Query Formulation in Information Retrieval.

2000-6 Rogier van Eijk (UU)

Programming Languages for Agent Communication

2000-7 Niels Peek (UU)

Decision-theoretic Planning of Clinical Patient Management

2000-8 Veerle Coup (EUR)

Sensitivity Analyis of Decision-Theoretic Networks

2000-9 Florian Waas (CWI)

Principles of Probabilistic Query Optimization

2000-10 Niels Nes (CWI)

Image Database Management System Design Considerations,

Algorithms and Architecture

2000-11 Jonas Karlsson (CWI)

Scalable Distributed Data Structures for Database Management

2001

2001-1 Silja Renooij (UU)

Qualitative Approaches to Quantifying Probabilistic Networks

2001-2 Koen Hindriks (UU)

Agent Programming Languages: Programming with Mental Models

2001-3 Maarten van Someren (UvA)

Learning as problem solving

2001-4 Evgueni Smirnov (UM)

Conjunctive and Disjunctive Version Spaces with

Instance-Based Boundary Sets

2001-5 Jacco van Ossenbruggen (VU)

Processing Structured Hypermedia: A Matter of Style

2001-6 Martijn van Welie (VU)

Task-based User Interface Design

2001-7 Bastiaan Schonhage (VU)

Diva: Architectural Perspectives on Information Visualization

2001-8 Pascal van Eck (VU)

A Compositional Semantic Structure for Multi-Agent Systems Dynamics.

2001-9 Pieter Jan 't Hoen (RUL)

Towards Distributed Development of Large Object-Oriented Models,

Views of Packages as Classes

2001-10 Maarten Sierhuis (UvA)

Modeling and Simulating Work Practice

BRAHMS: A Multiagent Modeling and Simulation Language

for Work Practice Analysis and Design

2001-11 Tom M. van Engers (VUA)

Knowledge Management:

The Role of Mental Models in Business Systems Design

2002 
2002-01 Nico Lassing (VU)

Architecture-Level Modifiability Analysis

2002-02 Roelof van Zwol (UT)

Modelling and searching web-based document collections

2002-03 Henk Ernst Blok (UT)

Database Optimization Aspects for Information Retrieval

2002-04 Juan Roberto Castelo Valdueza (UU)

The Discrete Acyclic Digraph Markov Model in Data Mining

2002-05 Radu Serban (VU)

The Private Cyberspace Modeling Electronic Environments

Inhabited by Privacy-concerned Agents

2002-06 Laurens Mommers (UL)

Applied Legal Epistemology;

Building a Knowledge-Based Ontology of the Legal Domain

2002-07 Peter Boncz (CWI)

Monet: A Next-Generation DBMS Kernel for Query-Intensive Applications

2002-08 Jaap Gordijn (VU)

Value Based Requirements Engineering: Exploring Innovative

e-Commerce Ideas

2002-09 Willem-Jan van den Heuvel(KUB)

Integrating Modern Business Applications with Objectified Legacy Systems

2002-10 Brian Sheppard (UM)

Towards Perfect Play of Scrabble

2002-11 Wouter C.A. Wijngaards (VU)

Agent Based Modelling of Dynamics: Biological and Organisational

Applications

2002-12 Albrecht Schmidt (Uva)

Processing XML in Database Systems

2002-13 Hongjing Wu (TUE)

A Reference Architecture for Adaptive Hypermedia Applications

2002-14 Wieke de Vries (UU)

Agent Interaction: Abstract Approaches to Modelling, Programming and

Verifying Multi-Agent Systems

2002-15 Rik Eshuis (UT)

Semantics and Verification of UML Activity Diagrams for Workflow Modelling

2002-16 Pieter van Langen (VU)

The Anatomy of Design: Foundations, Models and Applications

2002-17 Stefan Manegold (UVA)

Understanding, Modeling, and Improving Main-Memory Database Performance

2003

2003-01 Heiner Stuckenschmidt (VU)

Ontology-Based Information Sharing in Weakly Structured Environments 2003-02 Jan Broersen (VU)

Modal Action Logics for Reasoning About Reactive Systems

2003-03 Martijn Schuemie (TUD)

Human-Computer Interaction \& Presence in Virtual Reality Exposure Therapy 


\section{SIKS DISSERTATION SERIES}

2003-04 Milan Petkovic (UT)

Content-Based Video Retrieval Supported by Database Technology 2003-05 Jos Lehmann (UVA)

Causation in Artificial Intelligence and Law - A modelling approach 2003-06 Boris van Schooten (UT)

Development and Specification of Virtual Environments 2003-07 Machiel Jansen (UvA)

Formal Explorations of Knowledge Intensive Tasks

2003-08 Yongping Ran (UM)

Repair Based Scheduling

2003-09 Rens Kortmann (UM)

The Resolution of Visually Guided Behaviour

2003-10 Andreas Lincke (UvT)

Electronic Business Negotiation: Some Experimental Studies on the Interaction Between Medium, Innovation Context and Culture

2003-11 Simon Keizer (UT)

Reasoning under Uncertainty in Natural Language Dialogue using Bayesian

Networks

2003-12 Roeland Ordelman (UT)

Dutch Speech Recognition in Multimedia Information Retrieval

2003-13 Jeroen Donkers (UM)

Nosce Hostem - Searching with Opponent Models

2003-14 Stijn Hoppenbrouwers (KUN)

Freezing Language: Conceptualisation Processes across ICT-Supported

Organisations

2003-15 Mathijs de Weerdt (TUD)

Plan Merging in Multi-Agent Systems

2003-16 Menzo Windhouwer (CWI)

Feature Grammar Systems - Incremental Maintenance of Indexes to

Digital Media Warehouses

2003-17 David Jansen (UT)

Extensions of Statecharts with Probability, Time, and Stochastic Timing

2003-18 Levente Kocsis (UM)

Learning Search Decisions

\section{4}

2004-01 Virginia Dignum (UU)

A Model for Organizational Interaction: Based on Agents, Founded in Logic 2004-02 Lai Xu (UvT)

Monitoring Multi-Party Contracts for e-business

2004-03 Perry Groot (VU)

A Theoretical and Empirical Analysis of Approximation in Symbolic Problem

Solving

2004-04 Chris van Aart (UVA)

Organizational Principles for Multi-Agent Architectures

2004-05 Viara Popova (EUR)

Knowledge discovery and monotonicity 
2004-06 Bart-Jan Hommes (TUD)

The Evaluation of Business Process Modeling Techniques

2004-07 Elise Boltjes (UM)

Voorbeeldig Onderwijs; Voorbeeldgestuurd Onderwijs, een Opstap naar

Abstract Denken, Vooral voor Meisjes

2004-08 Joop Verbeek(UM)

Politie en de Nieuwe Internationale Informatiemarkt, Grensregionale

politiële gegevensuitwisseling en digitale expertise

2004-09 Martin Caminada (VU)

For the Sake of the Argument; Explorations into Argument-Based Reasoning

2004-10 Suzanne Kabel (UVA)

Knowledge-Rich Indexing of Learning-Objects

2004-11 Michel Klein (VU)

Change Management for Distributed Ontologies

2004-12 The Duy Bui (UT)

Creating Emotions and Facial Expressions for Embodied Agents

2004-13 Wojciech Jamroga (UT)

Using Multiple Models of Reality: On Agents who Know how to Play

2004-14 Paul Harrenstein (UU)

Logic in Conflict. Logical Explorations in Strategic Equilibrium

2004-15 Arno Knobbe (UU)

Multi-Relational Data Mining

2004-16 Federico Divina (VU)

Hybrid Genetic Relational Search for Inductive Learning

2004-17 Mark Winands (UM)

Informed Search in Complex Games

2004-18 Vania Bessa Machado (UvA)

Supporting the Construction of Qualitative Knowledge Models

2004-19 Thijs Westerveld (UT)

Using Generative Probabilistic Models for Multimedia Retrieval

2004-20 Madelon Evers (Nyenrode)

Learning from Design: Facilitating Multidisciplinary Design Teams

2005

2005-01 Floor Verdenius (UVA)

Methodological Aspects of Designing Induction-Based Applications

2005-02 Erik van der Werf (UM))

AI techniques for the game of Go

2005-03 Franc Grootjen (RUN)

A Pragmatic Approach to the Conceptualisation of Language

2005-04 Nirvana Meratnia (UT)

Towards Database Support for Moving Object data

2005-05 Gabriel Infante-Lopez (UVA)

Two-Level Probabilistic Grammars for Natural Language Parsing 2005-06 Pieter Spronck (UM)

Adaptive Game AI

2005-07 Flavius Frasincar (TUE)

Hypermedia Presentation Generation for Semantic Web Information Systems 


\section{SIKS DISSERTATION SERIES}

2005-08 Richard Vdovjak (TUE)

A Model-driven Approach for Building Distributed Ontology-based Web

Applications

2005-09 Jeen Broekstra (VU)

Storage, Querying and Inferencing for Semantic Web Languages

2005-10 Anders Bouwer (UVA)

Explaining Behaviour: Using Qualitative Simulation in Interactive Learning

Environments

2005-11 Elth Ogston (VU)

Agent Based Matchmaking and Clustering - A Decentralized Approach to

Search

2005-12 Csaba Boer (EUR)

Distributed Simulation in Industry

2005-13 Fred Hamburg (UL)

Een Computermodel voor het Ondersteunen van Euthanasiebeslissingen

2005-14 Borys Omelayenko (VU)

Web-Service Configuration on the Semantic Web; Exploring How Semantics

Meets Pragmatics

2005-15 Tibor Bosse (VU)

Analysis of the Dynamics of Cognitive Processes

2005-16 Joris Graaumans (UU)

Usability of XML Query Languages

2005-17 Boris Shishkov (TUD)

Software Specification Based on Re-usable Business Components

2005-18 Danielle Sent (UU)

Test-Selection Strategies for Probabilistic Networks

2005-19 Michel van Dartel (UM)

Situated Representation

2005-20 Cristina Coteanu (UL)

Cyber Consumer Law, State of the Art and Perspectives

2005-21 Wijnand Derks (UT)

Improving Concurrency and Recovery in Database Systems by Exploiting

Application Semantics

2006

2006-01 Samuil Angelov (TUE)

Foundations of B2B Electronic Contracting

2006-02 Cristina Chisalita (VU)

Contextual Issues in the Design and Use of Information Technology in

Organizations

2006-03 Noor Christoph (UVA)

The Role of Metacognitive Skills in Learning to Solve Problems

2006-04 Marta Sabou (VU)

Building Web Service Ontologies

2006-05 Cees Pierik (UU)

Validation Techniques for Object-Oriented Proof Outlines 2006-06 Ziv Baida (VU)

Software-aided Service Bundling - Intelligent Methods \& Tools 
for Graphical Service Modeling

2006-07 Marko Smiljanic (UT)

XML Schema Matching - Balancing Efficiency and Effectiveness by Means of

Clustering

2006-08 Eelco Herder (UT)

Forward, Back and Home Again - Analyzing User Behavior on the Web 2006-09 Mohamed Wahdan (UM)

Automatic Formulation of the Auditor's Opinion

2006-10 Ronny Siebes (VU)

Semantic Routing in Peer-to-Peer Systems

2006-11 Joeri van Ruth (UT)

Flattening Queries over Nested Data Types

2006-12 Bert Bongers (VU)

Interactivation - Towards an e-cology of People, our Technological

Environment, and the Arts

2006-13 Henk-Jan Lebbink (UU)

Dialogue and Decision Games for Information Exchanging Agents

2006-14 Johan Hoorn (VU)

Software Requirements: Update, Upgrade, Redesign - Towards a Theory of

Requirements Change

2006-15 Rainer Malik (UU)

CONAN: Text Mining in the Biomedical Domain

2006-16 Carsten Riggelsen (UU)

Approximation Methods for Efficient Learning of Bayesian Networks

2006-17 Stacey Nagata (UU)

User Assistance for Multitasking with Interruptions on a Mobile Device 2006-18 Valentin Zhizhkun (UVA)

Graph transformation for Natural Language Processing

2006-19 Birna van Riemsdijk (UU)

Cognitive Agent Programming: A Semantic Approach

2006-20 Marina Velikova (UvT)

Monotone Models for Prediction in Data Mining

2006-21 Bas van Gils (RUN)

Aptness on the Web

2006-22 Paul de Vrieze (RUN)

Fundaments of Adaptive Personalisation

2006-23 Ion Juvina (UU)

Development of Cognitive Model for Navigating on the Web

2006-24 Laura Hollink (VU)

Semantic Annotation for Retrieval of Visual Resources

2006-25 Madalina Drugan (UU)

Conditional log-likelihood MDL and Evolutionary MCMC

2006-26 Vojkan Mihajlovic (UT)

Score Region Algebra: A Flexible Framework for Structured Information

Retrieval

2006-27 Stefano Bocconi (CWI)

Vox Populi: Generating Video Documentaries from Semantically Annotated

Media Repositories

2006-28 Borkur Sigurbjornsson (UVA) 


\section{SIKS DISSERTATION SERIES}

Focused Information Access using XML Element Retrieval

\section{7}

2007-01 Kees Leune (UvT)

Access Control and Service-Oriented Architectures

2007-02 Wouter Teepe (RUG)

Reconciling Information Exchange and Confidentiality: A Formal Approach

2007-03 Peter Mika (VU)

Social Networks and the Semantic Web

2007-04 Jurriaan van Diggelen (UU)

Achieving Semantic Interoperability in Multi-agent Systems: A Dialogue-Based

Approach

2007-05 Bart Schermer (UL)

Software Agents, Surveillance, and the Right to Privacy: a Legislative

Framework for Agent-enabled Surveillance

2007-06 Gilad Mishne (UVA)

Applied Text Analytics for Blogs

2007-07 Natasa Jovanovic' (UT)

To Whom It May Concern - Addressee Identification in Face-to-Face Meetings

2007-08 Mark Hoogendoorn (VU)

Modeling of Change in Multi-Agent Organizations

2007-09 David Mobach (VU)

Agent-Based Mediated Service Negotiation

2007-10 Huib Aldewereld (UU)

Autonomy vs. Conformity: an Institutional Perspective on Norms and

Protocols

2007-11 Natalia Stash (TUE)

Incorporating Cognitive/Learning Styles in a General-Purpose Adaptive

Hypermedia System

2007-12 Marcel van Gerven (RUN)

Bayesian Networks for Clinical Decision Support: A Rational Approach to

Dynamic Decision-Making under Uncertainty

2007-13 Rutger Rienks (UT)

Meetings in Smart Environments; Implications of Progressing Technology

2007-14 Niek Bergboer (UM)

Context-Based Image Analysis

2007-15 Joyca Lacroix (UM)

NIM: a Situated Computational Memory Model

2007-16 Davide Grossi (UU)

Designing Invisible Handcuffs. Formal investigations in Institutions and

Organizations for Multi-agent Systems

2007-17 Theodore Charitos (UU)

Reasoning with Dynamic Networks in Practice

2007-18 Bart Orriens (UvT)

On the Development an Management of Adaptive Business Collaborations

2007-19 David Levy (UM)

Intimate Relationships with Artificial Partners

2007-20 Slinger Jansen (UU) 
Customer Configuration Updating in a Software Supply Network 2007-21 Karianne Vermaas (UU)

Fast Diffusion and Broadening Use: A Research on Residential Adoption and Usage of Broadband Internet in the Netherlands between 2001 and 2005 2007-22 Zlatko Zlatev (UT)

Goal-Oriented Design of Value and Process Models from Patterns 2007-23 Peter Barna (TUE)

Specification of Application Logic in Web Information Systems

2007-24 Georgina Ramrez Camps (CWI)

Structural Features in XML Retrieval

2007-25 Joost Schalken (VU)

Empirical Investigations in Software Process Improvement

\section{8}

2008-01 Katalin Boer-Sorbn (EUR)

Agent-Based Simulation of Financial Markets: A Modular, Continuous-Time Approach

2008-02 Alexei Sharpanskykh (VU)

On Computer-Aided Methods for Modeling and Analysis of Organizations 2008-03 Vera Hollink (UVA)

Optimizing Hierarchical Menus: A Usage-Based Approach

2008-04 Ander de Keijzer (UT)

Management of Uncertain Data - Towards Unattended Integration

2008-05 Bela Mutschler (UT)

Modeling and Simulating causal Dependencies on Process-Aware Information

Systems from a Cost Perspective

2008-06 Arjen Hommersom (RUN)

On the Application of Formal Methods to Clinical Guidelines, an Artificial

Intelligence Perspective

2008-07 Peter van Rosmalen (OU)

Supporting the Tutor in the Design and Support of Adaptive e-Learning 2008-08 Janneke Bolt (UU)

Bayesian Networks: Aspects of Approximate Inference

2008-09 Christof van Nimwegen (UU)

The Paradox of the Guided User: Assistance can be Counter-Effective

2008-10 Wauter Bosma (UT)

Discourse oriented summarization

2008-11 Vera Kartseva (VU)

Designing Controls for Network Organizations: A Value-Based Approach

2008-12 Jozsef Farkas (RUN)

A Semiotically Oriented Cognitive Model of Knowledge Representation

2008-13 Caterina Carraciolo (UVA)

Topic Driven Access to Scientific Handbooks

2008-14 Arthur van Bunningen (UT)

Context-Aware Querying; Better Answers with Less Effort

2008-15 Martijn van Otterlo (UT)

The Logic of Adaptive Behavior: Knowledge Representation and Algorithms for the Markov Decision Process Framework in First-Order Domains 


\section{SIKS DISSERTATION SERIES}

2008-16 Henriette van Vugt (VU)

Embodied Agents from a User's Perspective

2008-17 Martin Op 't Land (TUD)

Applying Architecture and Ontology to the Splitting and Allying of Enterprises

2008-18 Guido de Croon (UM)

Adaptive Active Vision

2008-19 Henning Rode (UT)

From Document to Entity Retrieval: Improving Precision and Performance

of Focused Text Search

2008-20 Rex Arendsen (UVA)

Geen Bericht, Goed Bericht. Een Onderzoek naar de Effecten van de Introductie van Elektronisch Berichtenverkeer met de Overheid op de Administratieve

Lasten van Bedrijven

2008-21 Krisztian Balog (UVA)

People Search in the Enterprise

2008-22 Henk Koning (UU)

Communication of IT-Architecture

2008-23 Stefan Visscher (UU)

Bayesian Network Models for the Management of Ventilator-Associated

Pneumonia

2008-24 Zharko Aleksovski (VU)

Using Background Knowledge in Ontology Matching

2008-25 Geert Jonker (UU)

Efficient and Equitable Exchange in Air Traffic Management Plan Repair using Spender-signed Currency

2008-26 Marijn Huijbregts (UT)

Segmentation, Diarization and Speech Transcription: Surprise Data Unraveled

2008-27 Hubert Vogten (OU)

Design and Implementation Strategies for IMS Learning Design

2008-28 Ildiko Flesch (RUN)

On the Use of Independence Relations in Bayesian Networks

2008-29 Dennis Reidsma (UT)

Annotations and Subjective Machines - Of Annotators, Embodied Agents,

Users, and Other Humans

2008-30 Wouter van Atteveldt (VU)

Semantic Network Analysis: Techniques for Extracting, Representing and

Querying Media Content

2008-31 Loes Braun (UM)

Pro-Active Medical Information Retrieval

2008-32 Trung H. Bui (UT)

Toward Affective Dialogue Management using Partially Observable Markov

Decision Processes

2008-33 Frank Terpstra (UVA)

Scientific Workflow Design; Theoretical and Practical Issues

2008-34 Jeroen de Knijf (UU)

Studies in Frequent Tree Mining

2008-35 Ben Torben Nielsen (UvT)

Dendritic Morphologies: Function Shapes Structure 
2009

2009-01 Rasa Jurgelenaite (RUN)

Symmetric Causal Independence Models

2009-02 Willem Robert van Hage (VU)

Evaluating Ontology-Alignment Techniques

2009-03 Hans Stol (UvT)

A Framework for Evidence-based Policy Making Using IT

2009-04 Josephine Nabukenya (RUN)

Improving the Quality of Organisational Policy Making using Collaboration

Engineering

2009-05 Sietse Overbeek (RUN)

Bridging Supply and Demand for Knowledge Intensive Tasks - Based on

Knowledge, Cognition, and Quality

2009-06 Muhammad Subianto (UU)

Understanding Classification

2009-07 Ronald Poppe (UT)

Discriminative Vision-Based Recovery and Recognition of Human Motion 2009-08 Volker Nannen (VU)

Evolutionary Agent-Based Policy Analysis in Dynamic Environments 2009-09 Benjamin Kanagwa (RUN)

Design, Discovery and Construction of Service-Oriented Systems

2009-10 Jan Wielemaker (UVA)

Logic Programming for Knowledge-Intensive Interactive Applications

2009-11 Alexander Boer (UVA)

Legal Theory, Sources of Law \& the Semantic Web

2009-12 Peter Massuthe (TUE, Humboldt-Universitaet zu Berlin)

Perating Guidelines for Services

2009-13 Steven de Jong (UM)

Fairness in Multi-Agent Systems

2009-14 Maksym Korotkiy (VU)

From Ontology-Enabled Services to Service-Enabled Ontologies (Making

Ontologies Work in e-Science with ONTO-SOA)

2009-15 Rinke Hoekstra (UVA)

Ontology Representation - Design Patterns and Ontologies that Make Sense 2009-16 Fritz Reul (UvT)

New Architectures in Computer Chess

2009-17 Laurens van der Maaten (UvT)

Feature Extraction from Visual Data

2009-18 Fabian Groffen (CWI)

Armada, An Evolving Database System

2009-19 Valentin Robu (CWI)

Modeling Preferences, Strategic Reasoning and Collaboration in

Agent-Mediated Electronic Markets

2009-20 Bob van der Vecht (UU)

Adjustable Autonomy: Controling Influences on Decision Making

2009-21 Stijn Vanderlooy (UM)

Ranking and Reliable Classification

2009-22 Pavel Serdyukov (UT) 


\section{SIKS DISSERTATION SERIES}

Search For Expertise: Going beyond Direct Evidence

2009-23 Peter Hofgesang (VU)

Modelling Web Usage in a Changing Environment

2009-24 Annerieke Heuvelink (VUA)

Cognitive Models for Training Simulations

2009-25 Alex van Ballegooij (CWI)

RAM: Array Database Management through Relational Mapping

2009-26 Fernando Koch (UU)

An Agent-Based Model for the Development of Intelligent Mobile Services

2009-27 Christian Glahn (OU)

Contextual Support of social Engagement and Reflection on the Web

2009-28 Sander Evers (UT)

Sensor Data Management with Probabilistic Models

2009-29 Stanislav Pokraev (UT)

Model-Driven Semantic Integration of Service-Oriented Applications

2009-30 Marcin Zukowski (CWI)

Balancing Vectorized Query Execution with Bandwidth-optimized Storage

2009-31 Sofiya Katrenko (UVA)

A Closer Look at Learning Relations from Text

2009-32 Rik Farenhorst (VU) and Remco de Boer (VU)

Architectural Knowledge Management: Supporting Architects and Auditors

2009-33 Khiet Truong (UT)

How Does Real Affect Affect Affect Recognition In Speech?

2009-34 Inge van de Weerd (UU)

Advancing in Software Product Management: An Incremental Method

Engineering Approach

2009-35 Wouter Koelewijn (UL)

Privacy en Politiegegevens; Over Geautomatiseerde Normatieve Informatieuitwisseling

2009-36 Marco Kalz (OU)

Placement Support for Learners in Learning Networks

2009-37 Hendrik Drachsler (OU)

Navigation Support for Learners in Informal Learning Networks

2009-38 Riina Vuorikari (OU)

Tags and Self-organisation: A Metadata Ecology for Learning Resources in a Multilingual Context

2009-39 Christian Stahl (TUE, Humboldt-Universitaet zu Berlin)

Service Substitution - A Behavioral Approach Based on Petri Nets

2009-40 Stephan Raaijmakers (UvT)

Multinomial Language Learning: Investigations into the Geometry of Language

2009-41 Igor Berezhnyy (UvT)

Digital Analysis of Paintings

2009-42 Toine Bogers (UvT)

Recommender Systems for Social Bookmarking

2009-43 Virginia Nunes Leal Franqueira (UT)

Finding Multi-step Attacks in Computer Networks using Heuristic Search and

Mobile Ambients 


Concerns such as identifying ways to control costs, improve quality, increase effectiveness, and manage risk have become increasingly important for organizations as they face more and more pressure to gain and maintain their competitive edge. Business-IT alignment (B-ITa) is recognized as a solution to these concerns.

There is a considerable literature on B-ITa in single organizations. Within this broad scope of literature, a number of authors have stressed the importance of assessing B-ITa in order to plan B-ITa improvement actions. In support of this, these authors have developed maturity models (MMs). However, B-ITa in collaborative networked organizations (CNOs) has hardly been studied. Yet, this is important because improved B-ITa entails a more efficient use of IT in the CNO supporting the integration of information systems and processes across organizational boundaries.

Notwithstanding the effective application of current B-ITa MMs for single organizations, to the best of our knowledge at the time of writing this dissertation, there is no $M M$ that specifically addresses the processes needed for achieving alignment between business and IT in CNOs. In response, this dissertation introduces the ICoNOs MM, a MM to assess B-ITa in CNOs. The ICoNOs MM presents a roll up of recommendations - e.g., coordination mechanisms, implementation strategies and organizational changes, in the form of process areas, specific goals and practices. Through its maturity levels, the ICoNOs MM provides improvement routes for those domains that are the most important for achieving alignment in CNOs. We believe that achieving B-ITa in CNOs is more complex than in single organizations because in collaborative settings, B-ITa is driven by goals of different independent organizations commonly with no centralized decision-making processes.

Throughout this dissertation, we present the results of four literature surveys, one focus group, and six case studies. Based on these conceptual and empirical research activities, we designed and validated the components underlying the ICONOs MM and the model itself.

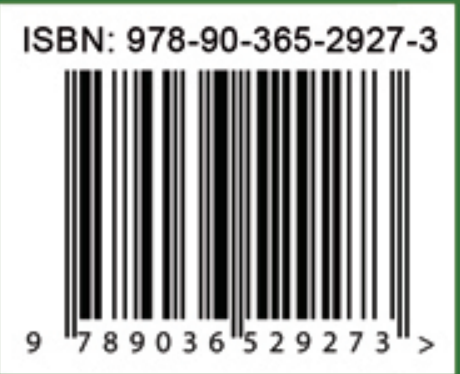

